AlII06 97861?

NBSIR 78-890

\title{
PLANE-WAVE SCATTERING-MATRIX THEORY OF ANTENNAS AND ANTENNA-ANTENNA INTERACTIONS
}

David M. Kerns

Electromagnetic Fields Division

Center for Electronics and Electrical Engineering

National Engineering Laboratory

National Bureau of Standards

Boulder, Colorado 80303

June 1978 


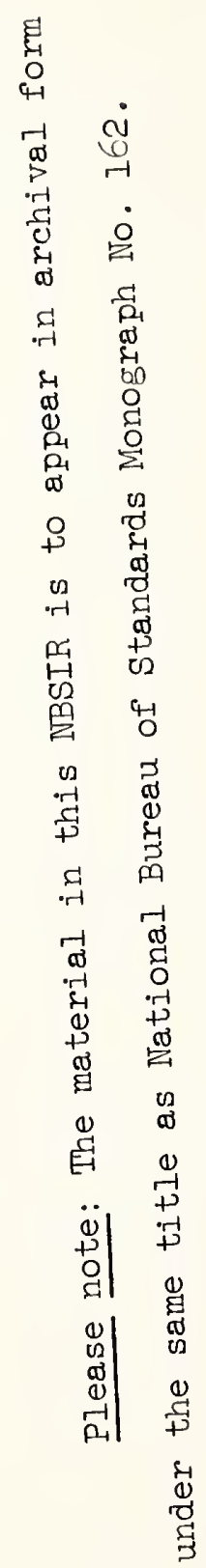


NBSIR 78-890

...

\section{PLANE-WAVE SCATTERING-MATRIX THEORY \\ OF ANTENNAS AND ANTENNA-ANTENNA INTERACTIONS}

David M. Kerns

Electromagnetic Fields Division

Center for Electronics and Electrical Engineering

National Engineering Laboratory

National Bureau of Standards

Boulder, Colorado 80303

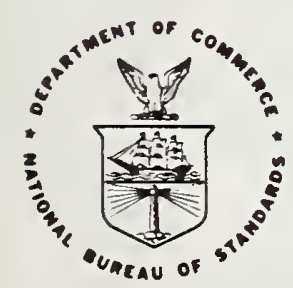

U.S. DEPARTMENT OF COMMERCE, Juanita M. Kreps, Secretary Sidney Harman, Under Secretary Jordan J. Baruch, Assistant Secretary for Science and Technology

NATIONAL BUREAU OF STANDARDS, Ernest Ambler, Director 


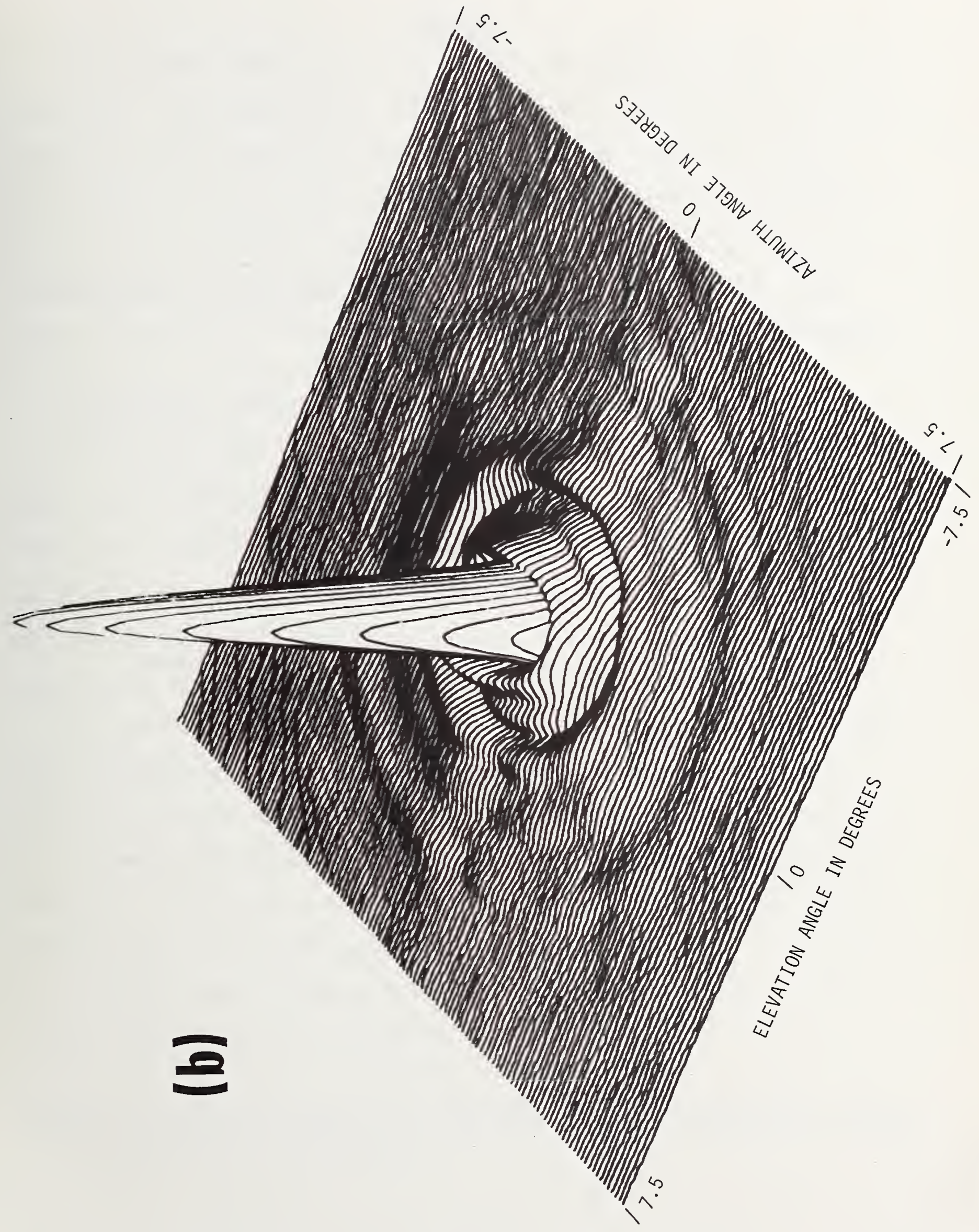




\section{FRONT ISPIECE}

(a) Forty-five cm diameter reflector antenna with Cassegrain feed and open ended waveguide probe.

Data were taken at a spacing of $0.508 \mathrm{~cm}$ (approximately 1 wavelength) in each direction in a transverse plane about $20 \mathrm{~cm}$ from the rim of the reflector. In this set-up bandlimiting was effectively due to the sharpness of the antenna pattern, so that half-wavelength spacing was not required.

(b) Far-field magnitude calculated for the arrangement shown.

Results were obtained over greatly magnified elevation and azimuth scales by a procedure devised by Allen C. Newell to utilize information available according to the sampling theorem but not provided by the unmodified FFT calculation. 


\section{PREFACE}

The rather cumbersome title of this monograph has been chosen advised1y. The monograph is distinguished by the use of plane-wave spectra for the representation of fields in space and by the consideration of antenna-antenna (and antenna-scatterer) interactions at arbitrary separation distances. The plane-wave representation is eminently suitable for this purpose as well as for the expressionof conventional asymptotic quantities of antenna theory, such as power gain, effective area, and polarization.

The primary objective of this monograph is to facilitate the critical acceptance and proper application of antenna and field measurement techniques deriving more or less directly from the plane-wave scattering-matrix (PWSM) theory of antennas and antennaantenna interactions. A secondary objective is to present some recent and some new theoretical results based on this theory. To some extent the second objective supports the first one.

Our expository plan is based upon our experience at NBS in developing, discussing, teaching ${ }^{1}$, and reporting on the PWSM theory and techniques as we 11 as upon observing at close hand the development of major NBS facilities for practical antenna measurement applications. We make no attempt to discuss the more recent, more complicated, and increasingly successful development of scatteringmatrix theory and techniques using cylindrical and spherical waves for the representation of spatial fields.

1 Much of the material of this monograph has been presented by the author at various stages of its development in graduate EE courses in the University of Colorado. 
All field and antenna measurements involve measurements of antema outputs and inputs. This part of the total process is the approximate dividing line between the domain of antenna theory and the domain of microwave measurement techniques including instrumentation. Or perhaps instead of a dividing line there is a gap or crevice.

Elaborate measurements made on expensive antennas with expensive measuring equipment can be (and have been) vitiated by improper microwave network calculations or by improper adjustments due to improper interpretation of impedance matching requirements. This is in part a reflection of omitted, inadequate, or even incorrect discussions of relatively simple network calculations in the literature.

One quantity, characteristic of the type of antenna measurements of interest here, is the transfer normalization required to obtain the ratio of receiving-antenna output to transinitting-antenna input corkectly in magnitude and relative phase. This normalization, even if done correctly with good instruments and good technique, may represent the single largest contribution to the inaccuracy or uncertainty of the eventually determined antenna or field data.

In short: To be able to make good microwave antenna measurements, one must be able to make good microwave measurements. This is true regardless of the brand of antenna theory that one uses.

The theoretical basis and the techniques of microwave network calculations receive little or no emphasis in books on electromagnetic theory and, apparently, surprisingly little in the training and background of microwave and microwave-antenna engineers.

\footnotetext{
"Microwave" is used as a convenient generic term, having appropriate connotations, but no frequency restriction is implicd.
} 
Chapter I of this monograph is intended to supply some of the missing emphasis. It consists of a revision and adaptation of part 1 of the book by Kerns and Beatty ${ }^{3}$ with an added section (adapted from Part 2 of the book) giving basic microwave network equations selected and discussed with antenna measurement applications in mind. The general theoretical foundation provided in this chapter has an additional role in the present monograph.

The use of the plane-wave representation of the spatial fields (as contrasted to the use of spherical or cylindrical waves) simplifies and strengthens the theoretical analogy between an antenna and an ordinary waveguide junction (or $\mathrm{N}$-port) described in terms of a finite number of discrete waveguide input or output modes, and it greatly simplifies the analogy between coupled antennas and coupled waveguide junctions.

In fact the PWSM description of the antennas was originally approached as a generalization of the theory of waveguide junctions, and this approach still seems the most expedient for brief qualitative introductions in talks and short courses. (A brief discussion of a discrete-mode model of coupled antennas is included in Chapter I.) The influence of this approach is quite apparent in Chapter II, less so in Chapter III.

Chapter $I$ is intended to be concise as well as elementary, but it is elementary only with respect to its principal subject. The reader is assumed to have a good working acquaintance with Maxwell's

Kerns, D.M., and Beatty, R.W., Basic Theory of Waveguide Junctions and Introductory Microwave Network Analysis (Pergamon Press, 1967). 
equations, vector analysis, and elementary matrix algebra. (For the remaindex of the monograph, these "prerequisites" should be supplemented by more vector-dyadic algebra (involving variable base vectors) and by acceptance of certain uses of 2 - and 3-dimensional Fourier transforms.) Transmission-line theory may be underrepresented in the discussion, but the use of the traveling wave resolution of fields more or less automatically takes care of the need for transmission-line equations.

Chapter II, in accordance with its title, gives a formulation of the PWSM theory of antennas and antenna-antcnna interactions together with basic analytical techniques for measurement applications. The formulation is relatively thorough and complete whereas the measurement techniques are treated briefly. The reason for this imbalance is that the measurement techniques are acquiring their own literature, to which references are given. Chapter II contains all of the material in the previous most complete discussion of the PWSM theory ${ }^{4}$, with revisions to improve the exposition and with added material making the work more complete and scholarly.

Perhaps the only feature of Chapter II that needs comment in this Preface is the degree of generality and the amount of detail in which we treat our subject. Our bias towards the subject of measurements as an end and not merely as a means is evident. By the same token we are interested in accurate measurements, or at

4 Kerns, 1).M., Plane-wave scattcring-matrix theory of antennas and antenna-antenna interactions: formulation and applications, J. Res. Nat. Bur. Stand. (U.S.), 80B, 5-51 (Jan. - Mar. 1976). 
least measurements with controlled approximations. Both generality and detajl are required to avoid over-idealization, to permit the framing of precise definitions, and to permit cognizance and the possibility of control of approximations. Less general theorems and simplified formulas might improve appearances of the pages of the monograph, but would tend to be much less convenient and useful to a measurement-oriented person interested in accurate measurements. It is much easier to reduce, than to increase, the generality of a given set of equations.

At any rate some of the generality is obtajned at relatively low, one-time cost, results in only slightly more complicated expressions, and yet yields much greater legitimate scope. In particular, this statement indicates the benefits of routinely admitting structures containing lossy, anisotropic media.

The generality of the PWSM formulation is such that we do not have to single out any particular types or classes of antennas for purposes of discussion of measurement equations. The measurement related theorems and equations are valid for arbitrary types of antennas, such as the so-called linear antennas, aperture antennas, and arrays of antenna elements. (Array theory per se is not considered.)

The author has experienced occasional difficulty in reading the literature and observed some difficulty in other people's apprehension of the content of this work attributable to contrasting basic assumptions - - or perhaps more precisely, to contrasting expectations regarding basic assumptions. For example, in the literature losslessness and reciprocity are frequently assumed 
(sometimes even tacitly), whereas in the present work media making up systems (not including transmission media) are in general lossy and non-reciprocal. Also, discussions in the literature often deal only with antenna power-pattern (a quantity proportional to the power-gain function, with no concern for the factor of proportionality); whereas (insofar as scalar description of transmitting characteristics is concerned) we are seriously interested only in power-gain functions (an absolute output/input type of quantity, in accordance with the IEEE Standard definition). Our assumptions are not tacit, but naturally in a fairly lengthy work there will be stretches without reminders or repetition. Perhaps these general remarks will result in improvement of the communication process in the present instance.

The third and last chapter is in essence a rather lengthy research paper, which, with considerable addjtional effort, could have been written as several shorter papers for separate publication. As it is written, it relies upon the first 3 of the $4 \mathrm{sec}-$ tions of chapter II for definitions, theorems, and motivation; in turn, the analytical results obtained in Chapter III support and illustrate Chapter II. Sufficient discussion of these interrelations, of the content, and of the origin of Chapter III is included in the chapter itself.

The use of examples is a feature of the exposition (38 numbered examples are included, most of them in Chapter III). As is usually the case, examples serve several purposes: The rederivation or restatement of certain well known results in the PWSM format is expected to lend perspective, establish confidence, and 
show some of the connections to existing theory. Examples are used to provide extensions of the text material not available or not readily available elsewhere, and to form part of the development of the subject. Most of the examples are suitable as material for exercises (but so also are many equations and assertions for which detailed argument is not given).

In this monograph the chapter is the main unit of composition. Each chapter has its own introduction, appendices, and literature references. Equations are numbered serially within subsections (or within sections that don't have subsections). References to equations are made by serial number alone within a given series; subsection or section and chapter numbers are supplied only as required for references going outside a given series.

\section{ACKNOWLEDGMENT}

It has been the author's good fortune to be associated for a good many years with members of strong groups (in the National Bureau of Standards) concerned with both theoretical and practical aspects of microwave and antenna measurements. The author and the monograph have benefitted immeasurably from this association. I would like to mention especially (in alphabetical order!) the following past or present associates: R.C. Baird, R.W. Beatty, E.S. Dayhoff, A.C. Newell, C.F. Stubenrauch, P.F. Wacker, and A.D. Yaghjian . 



\section{FOREWORD}

This monograph contains significant advances in antenna theory which provide new ways of analyzing antennas and antenna-antenna interactions, and which also serve as a foundation for important new antenna measurement techniques. It combines an excellent introduction to microwave network analysis (Chapter I) with a comprehensive treatment of the principles and applications of the plane-wave scattering-matrix theory of antennas (Chapters II and III). Thus, in one volume, the reader is provided with the basic information required to understand and perform a variety of accurate microwave antenna measurements (including, but not restricted to, near-field techniques) as well as the required supportive microwave circuit analyses and measurements. The materiảl presented has been developed over about the last twenty years (and much of it has been previously documented in various reports, publications, course notes, etc.). In this work the author combines the older material in a more comprehensive and polished form with significant new theoretical results (Chapter III).

The ability to perform accurate antenna measurements at reduced distances has been a long-sought goal of antenna engineers. Early attempts were at best inconclusive, generally because they were based on theoretical models applicable only to rather specialized cases or even on models insufficiently general for the experiments performed. During the late 1950's and early 1960's the author developed the first theory of sufficient scope to enable practical probe-corrected near-field measurements of broad classes of antennas. This work provides the basis for the accurate nearfield antenna measurement capabiljties developed at the National 
Burcau of Standards between about 1960 and 1970. These techniques, or variations of them, have since been adopted by several other organizations, and this monograph should hasten the due acceptance and application of these powerful new methods of measurement and analysis.

The accuracy of these new methods equals, or in some cases excecds, that achieved with the best far-field ranges, and more complete data are generally obtained. Admittedly, rather elaborate and expensive scanning facilities are required, but the costs are comparable to those incurred in the construction and equipping of far-field ranges--sometimes 1ess, sometimes more. The operational costs of a near-field facility may be less, and this is almost certainly true when detailed and extensive pattern information is required over large angular regions. Some other advantages of the near-field method are: (1) measurements can be performed in a laboratory enviromment free from the effects of weather, interference, and unwanted observation, (2) accurate absolute gain and polarization data are readily obtained, ( 3 ) near-field antenna interactions (e.g., mutual coupling) are treatable, and (4) rapid, extensive diagnostic testing of arrays and feeds is possible (a feature especially valuable during design stages) .

The development of the theory and applications described here is an excellent cxample of how research in one area can lead to unexpected, important results in another area. The plane-wave scattering-matrix (PWSM) formalism was initially developed by Drs. Dayhoff and Kerns to obtain a diffraction correction for a precise measurement of the speed of light using a microwave interferometer. 
Dr. Kerns subsequently realized that this "Diffraction Theory" constituted a generalization of existing antenna theory, including interactions, and could be used as a basis for near-field antenna measurements. The body of theory contained in this monograph and the experimental, data-processing, and computer techniques associated with planar near-field measurements are part of the outgrowth of that shift in emphasis. Others have used some of the basic concepts in developing non-planar scanning techniques, and the extrapolation method (used at NBS for high-accuracy on-axis gain and polarization measurements) is an extension of the PWSM approach. In addition, much of the PWSM theory has been reformulated for acoustics, and applications to optics and lasers have been suggested. It appears that extensions and new applications for this work will continue to be discovered and developed in several areas.

Some have expressed the view that this body of theory is too detailed and too complicated and, indeed, it may appear so when comparing these new techniques to simplified techniques that require no unfamiliar analytical tools. One should keep in mind, however, the fact that an underlying motivation of this work has been a means of performing reliable antenna measurements of specifiable and high accuracy. Such measurements can only be achieved by following careful experimental procedures that are based on a sound and complete theoretical foundation. The author has attempted to present sufficient theoretical detail and generality, coupled with the required physical insights and explanations, to enable others to apply this material to practical measurements. This "happy mediun" approach may be considered by some antemna enginecrs 
to be appallingly mathematical and rigorous, and by mathenaticians appallingly physical and non-rigorous. In any case, the book is noteworthy for its clarity of presentation and for the careful cxposition of important new concepts. For those who will expend the effort to become familiar with these new techniques, this monograph will become a valuable resource on the analysis and measurement of the function and interaction of electromagnetic radiators and transducers.

Boulder, Colorado

R.C. Baird

September, 1977 


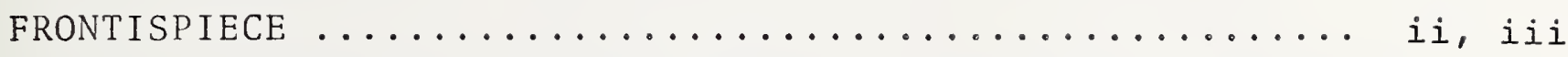

PREFACE $\ldots \ldots \ldots \ldots \ldots \ldots \ldots \ldots \ldots \ldots \ldots \ldots \ldots \ldots \ldots \ldots \ldots \ldots \ldots \ldots \ldots$

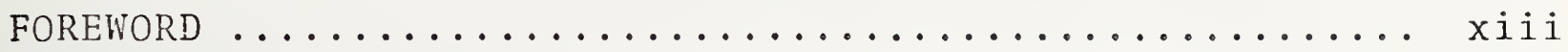

SOME CONVENTIONS AND NOTATION $\ldots \ldots \ldots \ldots \ldots \ldots \ldots \ldots \ldots \ldots \ldots \ldots \ldots \ldots$

CHAPTER I

MATRIX DESCRIPTION OF WAVEGUIDE N-PORTS AND ELEMENTARY NETWORK CALCULATIONS

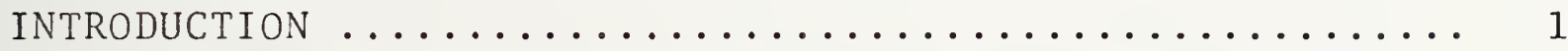

1. MAXWELL'S EQUATIONS: HARMONIC TIME DEPENDENCE, USE

of COMPLEX QUANTITIES .................. 4

2. INTRODUCTION OF HERTZ POTENTIALS: DERIVATION OF GENERAL SOLUTIONS FOR THE FIELDS IN WAVEGUIDE OF ARBITRARY CROSS SECTION ................... 9

2.1 Introduction of Hertz Potentials .......... 9

2.2 Calculation of Fields for Waveguide of Arbitrary Cross Section ................ 10

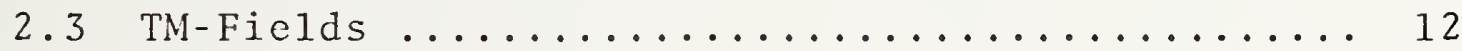

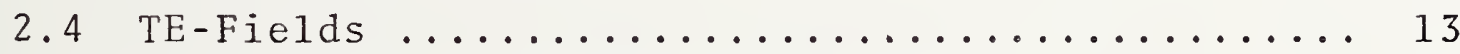

3. DisCussion OF THE EIgENVAlue PROBlems $\ldots \ldots \ldots \ldots \ldots$

3.1 General ........................... 14

3.2 Examples ........................... 15

3.3 General Properties of the Eigenvalue Problems... 18

4. DEFINITIONS OF $v$ AND $i$ FOR WAVEGUIDE MODES $\ldots \ldots \ldots 22$

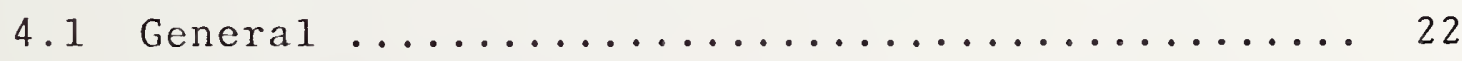

4.2 Basic Definitions and Relations .......... 23

4.3 Some Consequences of the Basic Definitions .... 27

Basis bields for the TE, mode in rectangular

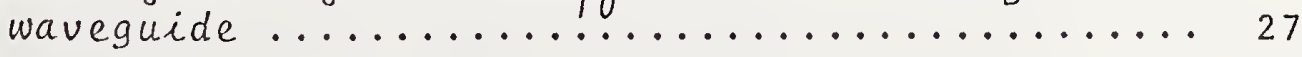

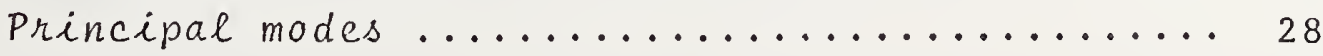


Dependence of $v_{a}, i_{a}$ upon $z \ldots \ldots \ldots \ldots . \ldots . \ldots . . .29$

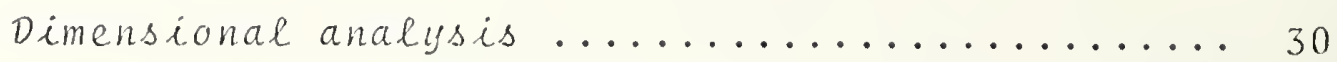

5. IMPEDANCE, ADMTTTANCF, AND SCATTERING MATRICES FOR

WAVEGUIDE JUNCTIONS .................. 32

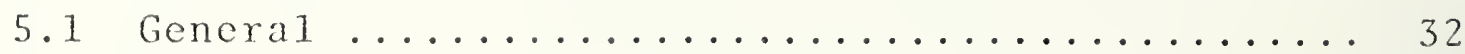

5.2 Definition of Impedance and Admittance Matrices. 32

5.3 The Scattering Natrix .............. 35

5.4 Power Re1ations ..................... 37

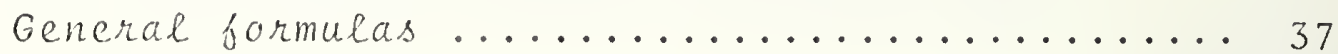

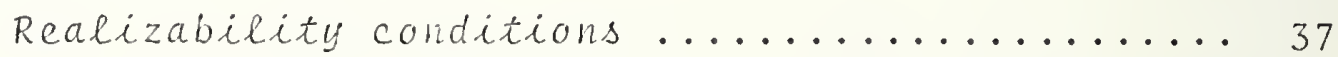

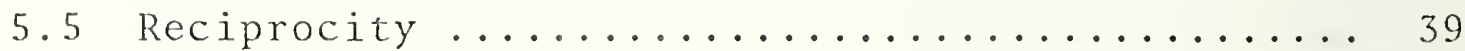

5.6 Change of Basis .................. 41

5.7 Sources; Joining Equations ............ 42

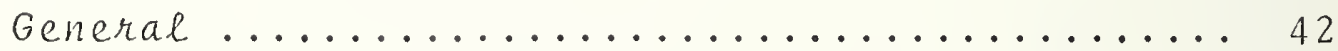

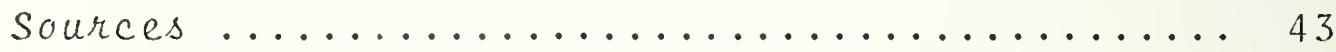

Joining equations ................... 44

6. NETWORK EQUATTONS ..................... 47

6.1 General ........................ 47

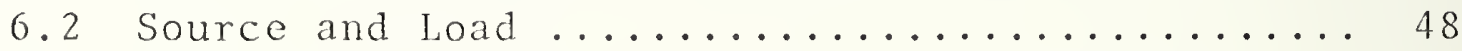

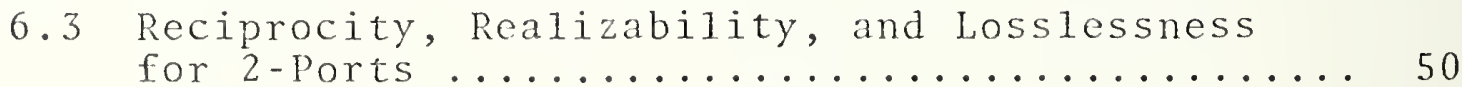

6.4 Two-Port Network Equations ............ 52

6.5 Discrete-Mode Model of Coupled Antcnnas ...... 58

7. APPENDICES TO CHAPTER I ................... 65

7.1 Appendix A: Modal Orthogonalities .......... 65

7.2 Appendix B: Characteristic Numbers of S ..... 67

7.3 Appendix C: Existence of S; Existence

Theorcms for $Z$ and $Y \ldots \ldots 68$ 
7.4 Appendix D: Formulas from Vector Analysis .... 70

7.5 Appendix E: Some Definitions and Rules

Concerning Matrices .............

7.6 Appendix F: Linear Dependence, Linear

Independence, and the Schwarz

Inequality for Complex Vectors ....

REFERENCES

\section{CHAPTER I I}

FORMULATION AND MEASUREMENT APPLICATIONS

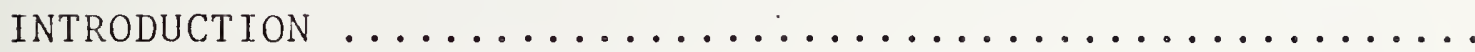

1. PLANE-WAVE SCATTERING-MATRIX FOR ANTENNAS AND

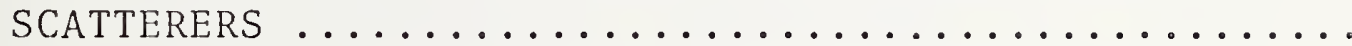

1.1 Representation of Fields on $\mathrm{S}_{0}$; Definition

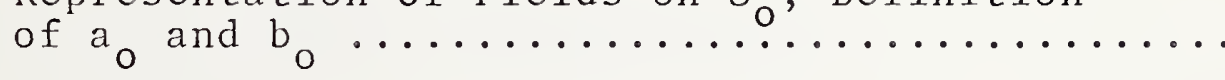

1.2 Representation of Fields in Space; Definition of $a_{q}(m, \underline{K})$ and $b_{q}(m, K) \ldots \ldots \ldots \ldots \ldots \ldots \ldots \ldots \ldots \ldots \ldots$

1.3 Definition of Scattering Matrices for

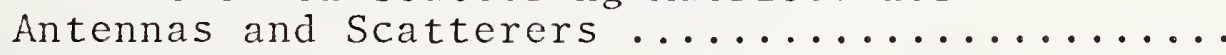

1.4 Power Expressions .................... 105

1.5 Reciprocity Relations--A Summary ........... 113

1.6 Relationships between PWSM Quantities and

Conventional Antenna Quantities .............

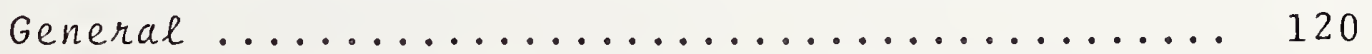

Power gain, effective area, and polarization

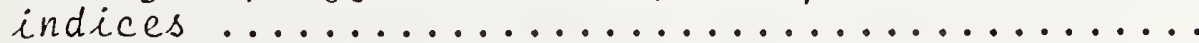

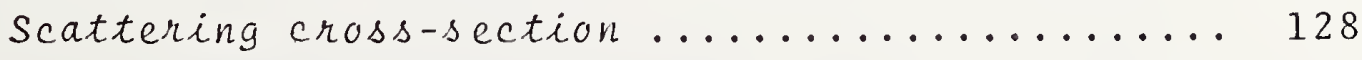

Generalized effective lengths ............... 130

2. SCATTERING-MATRIX ANALYSIS OF COUPLED ANTENNAS;

GENERAL SOLUTION FOR SYSTEM 2-PORT ............ 134

3. Determination OF COUPLing-PRODUCT VAlUES $\ldots \ldots \ldots \ldots 143$

3.1 Deconvolution of Transverse Scanning Data;

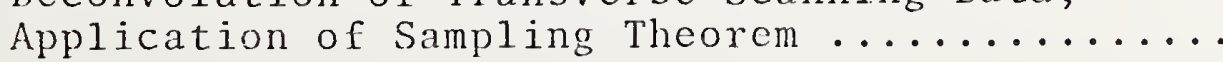

3.2 Received Signal as a Function of Distance and

the Extrapolation Technique 
4. UTILIZATION OF COUPLING-PRODUCT DATA $\ldots \ldots \ldots \ldots \ldots \ldots . .49$

4.1 One Unknown Antenna (Transmitting or Receiving). 149

4.2 Generalized Two-Identical-Antenna Techniques ... 153

4.3 Generalized Three-Antenna Techniques ........ 162

5. Appendices to CHAPter it $\ldots \ldots \ldots \ldots \ldots \ldots \ldots \ldots \ldots$

5.1 Appendix A: Reciprocity Theorems in the PWSM Context .............. 170

5.2 Appendix B: Dissipative Characteristics of Media; Comparison for Mutually Adjoint Media .............. 176

5.3 Appendix C: Generalized Joining Equations .... 179

5.4 Appendix D: Representation of Planar Scanning Equation as a Convolution ........ 182

5.5 Appendix E: Two-Dimensional, Spatial Sampling or Interpolation Theorem ........ 184

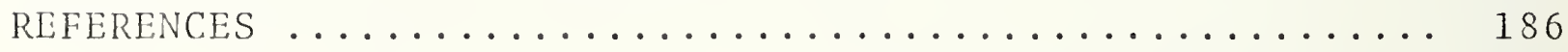

CHAPTER III

THEORETICAL DEVELOPMENT AND ANALYTICAL EXAMPLES

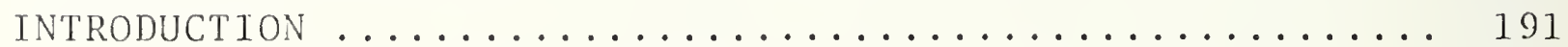

1. SYSTEMATIC INTRODUCTION OF THE VECTOR-DYADIC PWSM OF THE "SECOND KIND" .................... 194

2. SPECTRA OF ARBITRARY ANTENNAS, SCATTERERS, AND

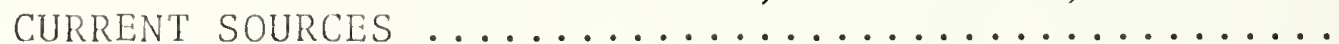

2.1 Formal Reduction of Antenna and Scattering Problems to Problems in Vacuo ............. 201

2.2 Spectra of Prescribed Currents in Vacuo ...... 203

3. SOME THEORY OF CONDITIONALLY NON-SCATTERING AND MINIMUM-SCATTERING ANTENNAS ...............

4. CLASSES AND EXAMPLES OF SOLVABLE TRANSMISSIONAND REFLECTION-SYSTEM PROBLEMS 
5. LAURENT SERIES ASSOCIATED WITH TRANSMISSION-

AND REFLECTION-INTEGRALS ................. 248

5.1 Expansion of $\exp (-i k r) \Psi_{0^{\prime} \mathrm{O}}(\underline{r})$ in Powers of $1 / \mathrm{r} \ldots 248$

5.2 Expansion of $\exp (-2 i k r) \Phi_{00}(\underline{r})$ in Powers of $1 / r \ldots 260$

6. APPENDICES TO CHAPTER III ................. 269

6.1 Appendix A: Ideal Electric-Probe Response ..... 269

6.2 Appendix B: Relation between Spectrum and

Asymptotic E .................. 271

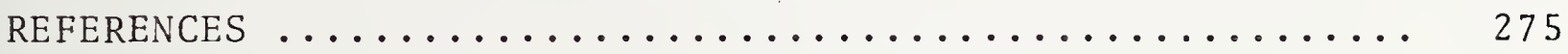





\section{(a) Conventions}

1. Complex numbers are sometimes called phasors; they are not called vectors.

2. Underlined symbols are vectors in space (ordinary space or wave-number space). Components may be complex numbers.

3. Doubly underlined symbols are tensors or dyadics.

4. Scalar and vector products of two vectors are denoted by $A \cdot B$ and $\mathrm{A} \times \underline{B}$, respectively. The scalar product of three vectors taken in the cyclic order $A, B, C$ is denoted by $[A B C]$. (A selection of formulas of vector ânalysis is given in appendix $D$ of Chapter I.

5. A superposed bar denotes the complex conjugate.

6. The magnitude of a complex number $z$ is denoted by $|z|$.

7. The squared magnitude of a vector $\underline{V}$ is defined by $\underline{V} \cdot \bar{V}$ and denoted by $|\underline{V}|^{2}$.

8. The "square" of a vector $V$ is defined by $V \cdot V$ and denoted by $\mathrm{V}^{2}$. Example: $\mathrm{k}^{2}=\omega^{2} \mu \varepsilon$ (see the list of symbols following).

9. The letter $x, y$, or $z$ used as a summation index takes on the values $x, y$, and $z$.

10. Definitions, notation, and rules for matrix algebra are given in appendix $E$ of Chapter $I$.

11. "Transverse" means perpendicular to the z-axis unless otherwise indicated.

12. "On-axis" refers to the z-axis of coordinates, not to an axis possibly suggested by antenna geometry.

13. The (suppressed) time dependence is $\exp (-i \omega t)$.

14. For the use of the superposed circumflex ("1"), see the latter part of subsection 1.3 in Chapter II.

(b) Roman Letters

$\underline{a}_{q}$ : Complete vectorial spectrum for $\underline{E}$ of incident field (p.92).

$\underline{A}_{q}$ : Transverse part of $\underline{a}_{q}(p \cdot 93)$. 
$a_{0}$ : Incident wave-amplitude in antenna feed transmission line or waveguide $(p .84)$.

$\underline{b}_{\mathrm{q}}$ : Complete vectorial spectrum for $\underline{E}$ of scattered or radiated field (p.92).

$\underline{B}_{\mathrm{q}}:$ Transverse part of $\underline{\mathrm{b}}_{\mathrm{q}}(\mathrm{p} .93)$.

$\mathrm{b}_{\mathrm{o}}$ : Emergent wave-amplitude in antenna feed transmission line or waveguide $(p .84)$.

dK: Symbolizes surface element in double integrals in $k_{x}, k_{y}$ space.

dR: Symbolizes surface element in double integrals in $x, y$ space.

dr: Symbolizes volume element in triple integrals in $x, y, z$ space.

E: "Electric field" (complex representation).

$E_{t}:$ Transverse part of $\underline{E}$.

$\underline{e}_{x},-_{y}, \underline{e}_{z}:$ Fixed, orthogonal, right-handed system of unit vectors. $\underline{e}_{\|}{ }^{\prime}{ }_{\perp}, \underline{e}_{k}:$ Orthogonal, right-handed system of unit vectors ticd to

$G_{q}(\underline{K}):$ Power-gain function evaluated in the direction of $\underline{k}$; $\mathrm{q}=1$ or 2 implies $\underline{\mathrm{k}}=\underline{\mathrm{k}}^{+}$or $\underline{\mathrm{k}}^{-}$, respectively (p. 121).

HE: "Magnetic field" (complex representation).

$\underline{H}_{t}:$ Transverse part of $\underline{H}$.

i: $\sqrt{-1}$.

$\underline{k}$ : Propagation vector; components $k_{x}, k_{y}, k_{z}$.

$\underline{k}^{ \pm}$: Propagation vector with $z$-component equal to $\pm \gamma$.

$\underline{K}$ : Transverse part of $\underline{k}$ ( $\underline{K}$ is chosen real in this work.)

$\mathrm{k}: \quad \sqrt{\underline{k} \cdot \underline{k}}=\omega \sqrt{\mu \varepsilon}$ (a real quantity in this work).

$\mathrm{K}: \quad \sqrt{\mathrm{K} \cdot \underline{\mathrm{K}}}$

$\mathrm{m}, \mathrm{n}$ : Index taking on values 1,2 and indicating association with unit vectors $\underline{\kappa}_{1}, \underline{\kappa}_{2}(p .89)$.

p,q: Index taking on values 1,2 and indicating association with regions to the "right" and to the "left" of an antenna or scatterer (p.90). 
r: Position vector (a real vector); components $x, y, z$.

$\underline{R}$ : Transverse part of $\underline{r}$.

$r$ : Magnitude of $\underline{r}$.

R: Magnitude of $\underline{R}$.

S$_{\text {oq }}:$ Transverse vectorial receiving characteristic (p.99); part of uniplanar PWSM (p.194).

$\underline{S}_{o q}:$ Complementary receiving characteristic (p.124); part of biplanar PWSM (p.195).

$\underline{S}_{p q}:$ Uniplanar dyadic scattering characteristic (pp.99,194)

$\underline{\underline{S}}_{\mathrm{pq}}$ : Biplanar dyadic scattering characteristic (p.195).

$\underline{S}_{q 0}:$ Transverse vectorial transmitting characteristic (p.99); part of uniplanar PWSM (p. 194).

$\underline{s}_{\text {qo }}$ : Complete transmitting characteristic (p. 121); part of biplanar PWSM ( $p .195)$.

$w_{q}(\underline{K})$ : Polarization index for incident plane-waves (p. 124).

$\mathrm{Y}_{0}: \sqrt{\varepsilon / \mu}$, wave admittance for simple plane-waves in medium with parameters $\varepsilon, \mu$.

\section{(c) Greek Letters}

$\gamma: \sqrt{k^{2}-k^{2}}$, taken positive when $k<k$, positive imaginary when $\mathrm{K}>\mathrm{k} ; \mathrm{k}_{\mathrm{z}}= \pm \mathrm{\gamma}(\mathrm{p} .88)$.

$\delta\left(k_{x}\right)$ : Dirac delta "function."

$\delta(\underline{K})$ : Abbreviation for $\delta\left(k_{x}\right) \delta\left(k_{y}\right)$.

$\varepsilon, \varepsilon_{0}:$ Permittivity of homogeneous, isotropic, dissipationless medium, usually space or vacuum.

$n_{0}:$ Characteristic admittance for the propagated mode in waveguide feed (p.84).

$n_{1}: \quad z$-component of wave-admittance for TM plane-waves in space; $n_{1}=\omega \varepsilon / \gamma=Y_{0} k / \gamma$, and for $0<\theta<\pi,=Y_{0} /|\cos \theta|$ (p. 89).

$n_{2}: \quad z$-component of wave-admittance for $T E$ plane-waves in space; $\eta_{2}=\gamma /(\omega \mu)=Y_{0} \gamma / k$, and for $0<\theta<\pi,=Y_{0}|\cos \theta|$ (p. 89). 
0: Polar angle in spherical polar coordinates (p. 95) in Chapters II and III.

$\underline{K}_{1}:$ Unit vector $\equiv \underline{K} / \mathrm{K}(\mathrm{pp}, 89,95)$.

$\underline{\kappa}_{2}:$ Unit vector $\equiv \underline{\mathrm{e}}_{2} \times \underline{\kappa}_{1}(\mathrm{pp} .89,95)$.

$\mu, \mu_{0}$ : Permeability of homogeneous, isotropic, dissipationless medium, usually space or vacuum.

$\rho_{0 q}$ : Polarization index for recciving characteristics (p. 125).

$\rho_{q 0}:$ Polarization index for transmitting characteristics (p. 122).

$\sigma_{\mathrm{q}}(\mathrm{K})$ : Effective area for reception (p.125); $\mathrm{q}=1$ or 2 implies $\underline{k}=\underline{k}^{-}$or $\underline{k}=\underline{k}^{+}$, respectively.

$\phi$ : Azimuthal angle of planc or spherical polar coordinates (p. 95) in Chapters II and III.

$\omega:$ Angular velocity as in the suppressed time factor $\exp (-i \omega t)$. 


\section{CHAPTER I}

\section{MATRIX DESCRIPTION OF WAVEGUIDE N-PORTS}

\section{AND ELEMENTARY NETWORK CALCULATIONS}

\section{INTRODUCTION}

As mentioned in the preface, Chapter I of this monograph offers an introduction to the impedance-, admittance-, and scattering-matrix descriptions of waveguide junctions. ${ }^{1}$ Some of the main ideas involved, with which the reader is assumed to be to some extent familiar, may be outlined as follows. A waveguide junction is defined as a linear electromagnetic system possessing ideal waveguide leads, which individually may be of arbitrary cross section. Coaxial transmission line, in particular, is considered as one form of waveguide and no restriction to microwave frequencies is implied. As special cases the concept of waveguide junction is applied to such things as straight sections of wavegide, terminations, and equivalent sources as well as to such things as directional couplers and hybrid junctions (which conform better to the connotation of the term "waveguide junction"). We regard a waveguide junction essentially as a "transducer," i.e., as a device for transferring power from a given mode in a given waveguide to a given mode in a given waveguide. In this chapter our main objective is to be able to describe a waveguide junction from this transducer point of view.

\footnotetext{
$\overline{1}$ For a supplementary discussion of a more qualitative and less general nature, see [1]. (Figures in brackets indicate the literature references at the end of this chapter.)
} 
The discussion is based directly on Maxwell's equations, and considers only the common and basic case of harmonic timedependence. The theory of ideal waveguide of arbitrary cross section is presented in concise and convenient form, and needed general properties, arising from general properties of the underlying eigenvalue problems, are summarized. "Voltage" and "current" are defined for waveguide modes. These definitions, together with the hypothesis of linearity, enable one to set up the desired descriptive scheme in terms of linear algebraic equations. The coefficients in these linear equations are the elements of the impedance, admittance, and scattering matrices of the waveguide junction.

These matrices characterize waveguide junctions for purposes of analysis or design of systems. The matrix elements themselves may become the object of experimental measurements or the subject of detailed theoretical calculations.

It will be clear that the matrix description of waveguide junctions represents a great simplification and abstraction from a detailed solution of Maxwell's equations, which indeed are al1-too-often impracticably difficult to solve. Fortunately, important information is derivable with relatively little labor from general hypotheses, such as reciprocity and realizability. A number of general relations and theorems--e.g., the realizability and reciprocity conditions for impedance, admittance, and scattering matrices--are derived briefly, with a view to bringing out the meaning of the scheme and providing reference material as well. Furthermore, basic network equations for interconnected sources, 
two-ports, and loads are presented and discussed with antenna measurement applications in mind. A discrete-mode model of coupled antennas, compatible with the PWSM formalism, is formulated and discussed.

Some features included in the present exposition are the introduction of explicit, separate, power and impedance normalizations; the observation of the occurrence of frequency-dependence in terminal basis fields (overlooked in one well-known book); the provision of a dimensional analysis (according to which, in one scheme, modal "voltage" and "current" come out in volts and amperes, respectively); and a precise classification of realizability conditions. 
1. MAXWELL'S EQUATIONS; HARMONIC TIME DEPENDENCE, USE OF COMPLEX QUANTITIES

We postulate Maxwell's equations,

$$
\begin{aligned}
& \nabla \times \underline{E}^{\prime \prime}+\frac{\partial \underline{B}^{\prime \prime}}{\partial t}=0, \\
& \nabla \times \underline{H}^{\prime \prime}-\frac{\partial \underline{D}^{\prime \prime}}{\partial t}-\underline{J}_{i}^{\prime \prime}=\underline{J}^{\prime \prime} .
\end{aligned}
$$

$\underline{E}$ " and $\underline{H}^{\prime \prime}$ are traditionally known respectively as the electric and magnetic intensities, $\underline{B}^{\prime \prime}$ is called the magnetic induction, and $\underline{D}^{\prime \prime}$ the electric displacement. $\underline{J}_{i}^{\prime \prime}$ is the induced current density, and $\underline{J}^{\prime \prime}$ is an enforced current density. Al1 these vectors are functions of position $\underline{r}$ and time t. (Rationalized MKS units are used throughout.)

We shall consider only the case in which all field quantities depend harmonically upon time, with frequency $\omega / 2 \pi$. (This case is in itself by far the most important one in the present context; moreover, arbitrary time dependence can be resolved into sinusoidal components by Fourier analysis.) This means, in the first place, that $\underline{J}^{\prime \prime}(\underline{r}, t)$ can be written

$$
\underline{J}^{\prime \prime}(\underline{r}, t)=\sum_{X}|J x(\underline{r})| \cos \left[\phi_{X}(\underline{r})-\omega t\right] \underline{e}_{X},
$$

where $x$ (or $y$ or $z$ ) used as a summation index runs over $x, y, z$ and $\underline{e}_{x}, \underline{e}_{y}$, and $\underline{e}_{z}$ are the unit vectors of a rectangular coordinate system 0xyz. Further 


$$
\begin{aligned}
\underline{J}^{\prime \prime}(\underline{r}, t) & =\operatorname{Re} \sum_{x}|J x \underline{r}(\underline{r})| e^{i \phi_{x}(\underline{r})} \underline{e}_{x} e^{-i \omega t} \\
& \equiv \operatorname{Re}\left[\underline{J}(\underline{r}) e^{-i \omega t}\right]
\end{aligned}
$$

--defining the vector $\underline{J}$, which in general has complex numbers for its $x, y, z$ components. For convenience we define $\underline{J}^{\prime}=\underline{J} \exp (-i \omega t)$. Next, assuming that all the vectors in (1) will possess harmonic time dependence, so that we may write $\underline{E}^{\prime \prime}(\underline{r}, t)=\operatorname{Re}[\underline{E}(r) \exp (-i \omega t)]=\operatorname{Re}\left[\underline{E}^{\prime}(\underline{r}, t)\right]$, etc., we consider the following equations

$$
\begin{gathered}
\nabla \times \underline{E}^{\prime}+\frac{\partial \underline{B}^{\prime}}{\partial t}=0, \\
\nabla \times \underline{H}^{\prime}-\frac{\partial \underline{D}^{\prime}}{\partial t}-\underline{J}_{i}^{\prime}=\underline{J}^{\prime} .
\end{gathered}
$$

Note that if we take real parts, (2) goes back to (1). Equation 2, which can be thought of as the superposition of (1) for prescribed current density vectors $\operatorname{Re}\left(\underline{J}^{\prime}\right)$ and $i \operatorname{Im}\left(\underline{J}^{\prime}\right)$, is introduced because it enables us to solve for time-dependence once and for all, and in fact eliminate the time factor from the equations. Thus, performing the differentiations with respect to $t$ and cancelling the common factor $\exp (-i \omega t)$, we arrive at

$$
\begin{aligned}
\nabla \times \underline{E}-i \omega \underline{B} & =0, \\
\nabla \times \underline{H}+i \omega \underline{D}-J_{i} & =\underline{J} .
\end{aligned}
$$

If $\underline{E}, \underline{B}$, etc., satisfy these equations, then $\underline{E}^{\prime}, \underline{B}^{\prime}$, etc., will satisfy (2), and $\operatorname{Re}\left(\underline{E}^{\prime}\right), \operatorname{Re}\left(\underline{B}^{\prime}\right)$, etc., will satisfy (1). 
Thus far nothing has been said about the relations between $\underline{B}$ and $\underline{H}$, between $\underline{D}$ and $\underline{E}$, and between $\underline{J}_{i}$ and $\underline{E}$. We put

$$
\begin{aligned}
& \underline{B}=\mu \underline{H}, \\
& \underline{D}=\varepsilon \underline{E}, \\
& \underline{J}_{i}=\sigma \underline{E},
\end{aligned}
$$

where $\mu, \varepsilon, \sigma$ are respectively permeability, permittivity, and conductivity. For the time being, we need explicitly consider only isotropic media, so that here $\mu, \varepsilon$, and $\sigma$ are scalars. For material media these quantities depend upon $\omega$ and in general wili depend upon $\underline{r}$. These parameters are not to depend upon

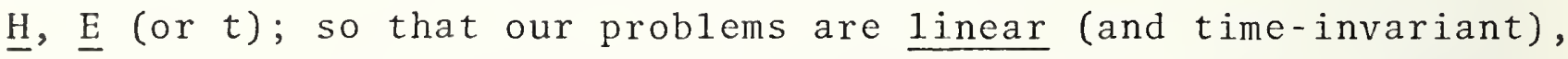
and we may assume that the impressed frequency $\omega / 2 \pi$ is the only frequency involved in the problem. Using the above "constitutive relations," we get finally,

$$
\begin{aligned}
\nabla \times \underline{E}-i \omega \mu \underline{H} & =0 \\
\nabla \times \underline{H}+i \omega \varepsilon \underline{E}-\sigma \underline{E} & =\underline{J} .
\end{aligned}
$$

This may be considered the working form of Maxwe11's equations for purposes of this chapter. As we have written the equations (with $\underline{J} \neq 0$ ) they are "inhomogeneous"; most of the time we will be using "homogeneous" equations, where $\mathrm{J}=0$ in the region under consideration.

Although $\underline{E}$ and $\underline{H}$ perhaps might be called "complex vector field amplitudes," we shall call them simply electric and magnetic fields, respectively. 
We sha11 state the most important energy relations in terms of $\underline{E}$ and $\underline{H}$.

Consider the real, instantaneous Poynting's vector:

$$
\begin{aligned}
\underline{P}(\underline{r}, t) & =\underline{E}^{\prime \prime}(r, t) \times \underline{H}^{\prime \prime}(\underline{r}, t) \\
& =\operatorname{Re}\left[\underline{E}(\underline{r}) e^{-i \omega t}\right] \times \operatorname{Re}\left[\underline{H}(\underline{r}) e^{-i \omega t}\right] \\
& =\left[\sum_{x}\left|\underline{E}_{x}(\underline{r})\right| \cos \left(\alpha_{x}-\omega t\right) \underline{e}\right) \times\left(\sum_{y}\left|H_{y}(\underline{r})\right| \cos \left(\beta_{y}-\omega t\right) \underline{e}_{y}\right) \\
& =\left[\sum_{y}\left|E_{x}(\underline{r})\right|\left|H_{y}(\underline{r})\right| \cos \left(\alpha_{x}-\omega t\right) \cos \left(\beta_{y}-\omega t\right)\left(\underline{e}_{x} \times \underline{e}_{y}\right) .\right.
\end{aligned}
$$

The time average of this quantity is seen to be

$$
<\underline{P}(\underline{r}, t)>=\frac{1}{2} \sum_{x, y}\left|E_{x}(\underline{r})\right|\left|H_{y}(\underline{r})\right| \cos \left(\alpha_{x}-\beta_{y}\right)\left(\underline{e}_{x} \times \underline{e}_{y}\right) .
$$

Here the angular parentheses indicate time-average; a superposed bar will be used to indicate complex conjugate.

The complex Poynting's vector is defined as

$$
\begin{aligned}
& \underline{S}(\underline{r})=\frac{1}{2} \underline{E}(\underline{r}) \times \underline{\bar{H}}(\underline{r}) \\
& =\frac{1}{2} \sum_{x, y}\left|E_{x}(\underline{r})\right| e^{j \alpha} x\left|H_{y}(\underline{r})\right| e^{-j \beta} y\left(\underline{e}_{x} \times \underline{e}_{y}\right) \text {. }
\end{aligned}
$$

Hence the real part of the complex Poynting's vector is equal to the time average of the real, instantaneous Poynting 's vector:

$$
\operatorname{Re}[\underline{S}(\underline{r})]=\langle\underline{P}(\underline{r}, t)\rangle \text {. }
$$

The complex Poynting's vector has further important properties. If we compute $\nabla \cdot \underline{S}(\underline{r})$, we get

$$
\nabla \cdot \underline{S}(\underline{r})=-\frac{1}{2} \sigma \underline{E} \cdot \bar{E}+i \omega\left(\frac{\mu}{2} \underline{H} \cdot \underline{H}-\frac{\varepsilon}{2} \underline{E} \cdot \underline{E}\right)
$$

in a region where $\underline{J}=0$. (Note that the scalar product of a vector with its complex conjugate is a real, non-negative 
quantity.) The instantaneous dissipation per unit volume due to the induced current $\underline{J}_{i}^{\prime \prime}(\underline{r}, t)=\sigma \underline{E}^{\prime \prime}(\underline{r}, t)$ is $\sigma\left[\underline{E}^{\prime \prime}(\underline{r}, t)\right]^{2}$; the rea1 part of the right-hand side of (6) is evidently the time average of this quantity. Instantaneous magnetic and electric energy densities are

$$
\begin{aligned}
& u_{H}(\underline{r}, t)=\frac{1}{2} \mu\left[\underline{H}^{\prime \prime}(\underline{r}, t)\right]^{2}, \\
& u_{E}(\underline{r}, t)=\frac{1}{2} \varepsilon\left[\underline{E}^{\prime \prime}(\underline{r}, t)\right]^{2} .
\end{aligned}
$$

Taking time averages yields

$$
\begin{aligned}
& \left\langle u_{H}(\underline{r}, t)\right\rangle=\frac{1}{4} \mu \underline{H}(\underline{r}) \cdot \bar{H}(\underline{r}), \\
& \left\langle u_{E}(\underline{r}, t)\right\rangle=\frac{1}{4} \varepsilon \underline{E}(\underline{r}) \cdot \bar{E}(\underline{r}) .
\end{aligned}
$$

Thus the imaginary part of the right-hand side of (6) is $2 \omega$ times $<u_{H}-u_{E}>$. 
2. INTRODUCTION OF HERTZ POTENTIALS; DERIVATION

OF GENERAL SOLUTIONS FOR THE FIELDS IN WAVEGUIDE

OF ARBITRARY CROSS SECTION

2.1 Introduction of Hertz Potentials

Waveguide fields are here conveniently derived from Hertz potentials. For our purposes a brief, self-contained treatment of the Hertz potentials is possible and will serve to introduce them.

We wish to solve Maxwell's equations in the form

$$
\begin{aligned}
& \nabla \times \underline{E}-i \omega \mu \underline{H}=0, \\
& \nabla \times \underline{H}+i \omega \varepsilon \underline{E}=0
\end{aligned}
$$

in a region in which $\mu$ and $\varepsilon$ are constant. We assert that if the vector II (a Hertz vector) satisfies the vector wave equation,

$$
\nabla^{2} \underline{I}+k^{2} \underline{I I}=0
$$

where $k^{2}=\omega^{2} \mu \varepsilon$, then the $\underline{E}$ and $\underline{H}$ given by

$$
\begin{aligned}
& \underline{E}=i \omega \mu \nabla \times \underline{I}, \\
& \underline{H}=\nabla \times \nabla \times \underline{I I}
\end{aligned}
$$

satisfy (1). It is easy to see that (1a) is satisfied. To verify (1b) we write

$$
\begin{aligned}
\nabla \times \underline{H} & =\nabla \times(\nabla \times \nabla \times \underline{I})=\nabla \times\left(\nabla \nabla \cdot \underline{I}-\nabla^{2} \underline{I}\right) \\
& =0+k^{2} \nabla \times \underline{I}=-i \omega \varepsilon \underline{E} .
\end{aligned}
$$


Moreover, another electromagnetic field is derivable from a II thru the equations

$$
\begin{aligned}
& \underline{E}=\nabla \times \nabla \times \underline{I}, \\
& \underline{H}=-i \omega \varepsilon \nabla \times \underline{I} .
\end{aligned}
$$

The verification is similar to the above and is omitted.

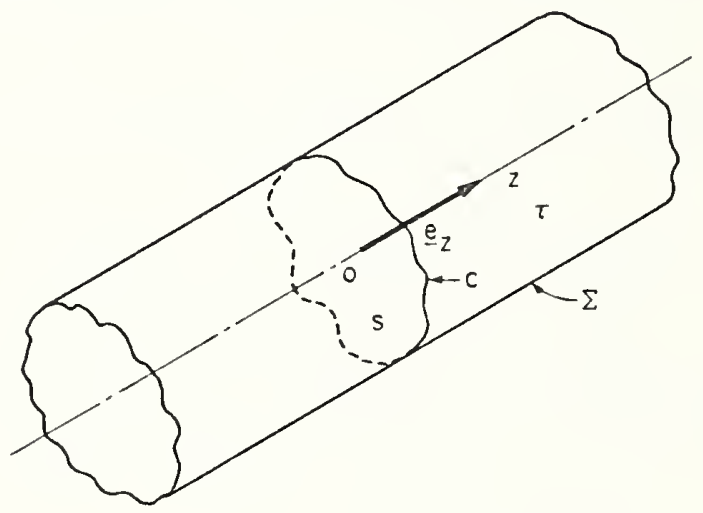

Fig. 1. Illustrating $\tau, \Sigma, S$, and $C$.

\subsection{Calculation of Fields for Waveguide of Arbitrary Cross Section}

The domain of the electromagnetic field in our waveguide is a cylindrical region $\tau$ bounded by a cylindrical surface $\Sigma$. A transverse plane (i.e., a plane perpendicular to the generators of $\Sigma$ ) cuts $\tau$ in a surface $S$ and $\Sigma$ in a curve $C$. The surface $S$, the cross-section of the waveguide, is to be a connected (but not necessarily simply-connected) region. The curve $\mathrm{C}$ forms the 
complete boundary of $\mathrm{S}$ and may consist of one or more distinct parts. (See Fig. 1.)

In $\tau$ the medium is to be homogeneous, isotropic, and (for "ideal" waveguide) non-dissipative. Conductivity in the medium, if present, may be accounted for by means of a complex $\varepsilon$. The walls of the waveguide coincide with $\Sigma$, and are to be perfectly conducting .

We take a $z$-axis parallel to the generators of $\Sigma$ and let $\underline{e}_{z}$ denote the unit vector in the $z$-direction. The transverse coordinates we leave unspecified.

We now assert that the waveguide fields may be derived from Hertz potentials of the form

$$
\underline{I}=\mathrm{f} \phi \underline{\mathrm{e}}_{\mathrm{Z}},
$$

where $f$ is a function only of the transverse coordinates and $\phi$ is a function only of $z$. (Evidence that we can get a complete set of solutions from II's of this form will appear later.) Since II must satisfy $(2.1-2), f$ and $\phi$ must satisfy

$$
-\frac{\nabla^{2} f}{f}=K^{2}=\frac{\phi^{\prime \prime}}{\phi}+k^{2} \text {, }
$$

where $\mathrm{K}^{2}$ is a separation constant. That is, we have the two differential equations

$$
\nabla^{2} f+K^{2} f=0, \phi^{\prime \prime}+\gamma^{2} \phi=0,
$$

where we have defined $\gamma^{2}=k^{2}-K^{2}$. The first is a two-dimensional scaiar wave equation (for the time-harmonic case). Its solutions are subject to boundary conditions to be determined below; at the moment we assume that $K^{2}$ will be real and non-negative. The second differential equation is a one-dimensional wave equation and its 
general solution may be written at once, viz.,

$$
\phi=A e^{i \gamma z}+B e^{-i \gamma z},
$$

where $A$ and $B$ are constants (independent of $\underline{r}$ ). If the medium is lossy, $\gamma$ will be complex and we shall put

$$
i \gamma=-\alpha+i \beta,
$$

where $\alpha$ and $\beta$ are both positive. For ideal waveguide, $\gamma$ is either pure real or pure imaginary, and we define

$$
\begin{aligned}
& r=\sqrt{k^{2}-k^{2}}=\beta, k^{2}>k^{2}, \\
& r=i \sqrt{k^{2}-k^{2}}=i \alpha, k^{2}<k^{2} .
\end{aligned}
$$

With these sign conventions, the exponentials exp( \pm irz) detcrmine fields traveling or attenuated in the $\pm z$-directions, respectively.

\subsection{TM-Fields}

With II of the above form we obtain from (2.1-4)

$$
\begin{aligned}
& \underline{E}=\phi^{\prime} \nabla f+K^{2} f \phi \underline{e}_{z}, \\
& \underline{H}=-i \omega \varepsilon \phi\left(\nabla f \times \underline{e}_{z}\right),
\end{aligned}
$$

omitting details of the calculation. Clearly, $\underline{H}^{\cdot} \underline{\mathrm{e}}_{\mathrm{z}}=0$, so that $\underline{H}$ is transverse; hence the designation "transverse magnetic" (TM). The basic boundary condition to be imposed is that the components of E tangent to $\Sigma$ vanish on $\Sigma$. This can be expressed as $\underline{\mathrm{n}} \times \underline{\mathrm{E}}=0$ on $\Sigma$, where $\underline{\mathrm{n}}$ is a normal unit vector on $\Sigma$. Two cases may be distinguished: (1) if $\mathrm{K}^{2} \neq 0$, it is necessary and sufficient that $f=0$ on the boundary $C$ of $S$ (in some cases supplomentary conditions such as single-valuedness or finiteness 
must be imposed). (2) If $K^{2}=0$, it is necessary and sufficient that $\underline{\mathrm{n}} \times \nabla f=0$ on $\mathrm{C}$.

It should be noted that non-trivial fields with $\mathrm{K}^{2}=0$, known as TEM (or principal) modes, can exist only under certain conditions. One such mode exists, for example, in the case of coaxial line, for which $S$ is an annulus (and is thus not simply connected). For finite regions, TEM modes exist if and only if the region is not simply connected (so that $f$ can have different constant values on the several parts of $\mathrm{C}$ ); the number of independent TEM modes is one less than the number of conductors. This statement applies essentially to infinite regions also, if the infinite "boundary" is counted as one conductor. As an example of an infinite region, one may consider the space outside a pair of conductors representing a two-wire transmission line, for which two TEM modes exist.

\subsection{TE-Fields}

Equation (2.1-3) yields "transverse electric" modes. For this case let us put $\underline{\underline{I}}=g \phi \underline{e}_{z}$, using "g" instead of "f." because g will be subject to different boundary conditions. From $(2.1-3)$ we then obtain

$$
\begin{aligned}
& \underline{E}=i \omega \mu \phi\left(\nabla g \times \underline{e}_{z}\right), \\
& \underline{H}=\phi^{\prime} \nabla g+K^{2} g \phi \underline{e}_{z} .
\end{aligned}
$$

The basic boundary condition, $\underline{\mathrm{n}} \times \underline{\mathrm{E}}=0$ on $\Sigma$, requires $\underline{\mathrm{n}} \cdot \nabla \mathrm{g}=0$ on $\mathrm{C}$ of $\mathrm{S}$. It can be shown that in the TE case there are no non-trivial electromagnetic fields for $K^{2}=0$. 


\section{DISCUSSION OF THE EIGENVALUE PROBLEMS}

\section{$3.1 \quad$ Genera1}

In the above expressions for the TM and TE modes the functions $f$ and $g$ (and the parameter $K^{2}$ ) are as yet undetermined. The determination of these functions and their properties is the main mathematical part of the theory of waveguides involved here.

The functions $f, g$ are subject to the 2 -dimensional waveequation and the boundary corditions found in connection with (2.3-1) and 2.4-1). In particular, the TEM f-functions are subject to

$$
\nabla^{2} f=0 \text { in } S, \underline{n} \times \nabla f=0 \text { on } C .
$$

The problem presented is essentially similar to two-dimensional problems in electrostatics or potential theory and is of a rather different character from that presented by. the other $f$ and $g$ functions .

The TM f's and the g's are subject to

$$
\begin{aligned}
& \nabla^{2} f+K^{2} f=0 \text { in } S, f=0 \text { on } C, \\
& \nabla^{2} g+K^{2} g=0 \text { in } S, \underline{n} \cdot \nabla g=0 \text { on } C .
\end{aligned}
$$

It should be observed that, since the above equations (including 1) are homogeneous, solutions are indeterminate at least to the extent of constant multipliers. "Distinct" solutions will always be understood to be linearly independent solutions. (Linear dependence and independence are defined in Appendix F.) Equations and (3) each represent what are called "eigenvalue problems," which are characterized by the following property: Non-trivial 
solutions (called eigenfunctions) exist only for certain values of the parameter $\mathrm{K}^{2}$ (called eigenvalues). (Sometimes the terms "characteristic functions" and "characteristic values" are used.) The eigenvalues as well as the eigenfunctions are in general different for (2) and (3); these are distinct and separate problems.

\subsection{Examples}

In a few cases (of which it seems that most are important) $f, g$, and $K^{2}$ can be found readily.

E.g., if $S$ is the (inside of a) rectangle bounded by the lines $x=0$, $a$ and $y=0, b$ in the coordinate system $0 x y$, the assumption $f=X(x) Y(y)$ in the TM problem leads to

$$
\begin{aligned}
& f_{m n}=\sin (m \pi x / a) \sin (n \pi y / b), \\
& K_{m n}^{2}=(m \pi / a)^{2}+(n \pi / b)^{2},
\end{aligned}
$$

where $m, n$ are positive integers. In the TE problem, the same assumption for g leads to

$$
\begin{aligned}
& g_{m n}=\cos (m \pi x / a) \cos (n \pi y / b), \\
& K_{m n}^{2}=(m \pi / a)^{2}+(n \pi / b)^{2},
\end{aligned}
$$

where $\mathrm{m}, \mathrm{n}$ are non-negative integers not both zero. The g-functions differ from the $f$-functions because the associated boundary conditions differ. For the rectangular region it happens that the eigenvalues for TE- and for TM-modes coincide for $m>1, n>1$; but this sort of wholesale coincidence does not in general occur. There is no TEM mode for the rectangle: Equation (3.1-1) has the solution $f=$ const., which is not trivial, but this leads to the trivial electromagnetic field $\underline{E}=0, \underline{H}=0$. 
Probably the case of circular waveguide is next in order of complexity. In this case, it is appropriate to take plane polar coordinates $\rho, \theta$ as the coordinates in the transverse plane. For $S$ we take the region bounded by the circle $\rho=a$. In the new coordinates the two-dimensional wave equation take the form

$$
\frac{1}{\rho} \frac{\partial}{\partial \rho}\left(\rho \frac{\partial u}{\partial \rho}\right)+\frac{1}{\rho^{2}} \frac{\partial^{2} u}{\partial \theta^{2}}+K^{2} u=0
$$

The assumption $u=R(\rho) F(\theta)$ leads to the separate ordinary equations

$$
\begin{array}{r}
F^{\prime \prime}+m^{2} F=0, \\
\rho\left(\rho R^{\prime}\right)^{\prime}+\left(K^{2} \rho^{2}-m^{2}\right) R=0,
\end{array}
$$

where $\mathrm{m}^{2}$ is the separation constant. From these equations it follows that one-valued, continuous solutions of (3) are

$$
u=J_{m}(K \rho) \frac{\sin m \theta,}{\cos m}
$$

where $m$ is an integer $(\overline{>} 0)$ and $J_{m}$ is the Bessel function of the first kind and $m^{\text {th }}$ order. (Eq. (5) represents two linearly independent solutions for each $\mathrm{m}>0$.) We obtain f-functions by imposing the boundary condition $u=0$ at $\rho=a, v i z$,

$$
f_{m n}=J_{m}\left(K_{m n} \rho\right) \underset{\cos }{\sin \theta}
$$

where $K_{m n}=x_{m n} / a$, and $x_{m n}$ is the $n^{\text {th }}$ positive root of $J_{m}(x)=0$. Similar1y, we obtain g-functions by imposing $\underline{n} \cdot \nabla g=0$ at $\rho=a$. Thus,

$$
g_{m n}=J_{m}\left(K_{m n}^{\prime} \rho\right) \underset{\cos }{\sin \theta}
$$


where $K_{m n}^{\prime}=x_{m n}^{\prime} / a$, and $x_{m n}^{\prime}$ is the $n^{\text {th }}$ positive root of $J_{m}^{\prime}(x)=0$.

In each of (6) and (7), two linearly independent eigenfunctions "belong to" any given eigenvalue with $\mathrm{m}>0$. These eigenvalues are said to be "degenerate." Degeneracy higher than twofold, when it occurs, must be regarded as "accidental"--the term being both technical and appropriate. When $m=0$, the eigenfunctions are circularly symmetric and non-degenerate (except for accidental degeneracies).

The electromagnetic fields E, $\underline{H}$ as distinguished from the functions $f, g$ ) of a TE- and a TM mode for which the respective eigenvalues happen to be equal may be thought of, and are referred to, as mutually aegenerate. (We have already noted the wholesaie degeneracy of this type in the case of a rectangular region.)

The lowest eigenvalue in the problem of circular waveguide occurs in the TE case with $m=n=1$ (see Table 1). According to (7), this $\mathrm{TE}_{11}$-eigenvalue is doubly degenerate. The accidental degeneracy of the $\mathrm{TE}_{0 \mathrm{n}}$ and the $\mathrm{TM}_{1 \mathrm{n}}$ modes is exhibited in the table for $n=1,2$.

TABLE 1 .

Some zeros of $J_{m}$ and $J_{m}^{\prime}$

$\begin{array}{lll} & \underline{\mathrm{n}=1} & \underline{\mathrm{n}=2} \\ \mathrm{~J}_{0} & 2.405 & 5.520 \\ \mathrm{~J}_{1} & 3.832 & 7.016 \\ \mathrm{~J}_{2} & 5.136 & 8.417 \\ \mathrm{~J}_{0}^{\prime} & 3.832 & 7.016 \\ \mathrm{~J}_{1}^{\prime} & 1.841 & 5.331 \\ \mathrm{~J}_{2}^{\prime} & 3.054 & 6.706\end{array}$


An important case in which a TEM mode cxists is, as already noted, that of coaxial line. We shall find the TEM $f$-function in this case. We use the same coordinates as in the preceding example and take $S$ to be the region between the circles $\rho=\mathrm{a}$ and $\rho=\mathrm{b}$. The desired function is readily found if we look for a solution that is circularly symmetric. Thus, putting $f=R(\rho)$ and using (3) (with $\left.K^{2}=0\right)$, we find

$$
\begin{aligned}
\left(\rho R^{\prime}\right)^{\prime} & =0, \\
R^{\prime} & =C_{1} / \rho,
\end{aligned}
$$

or $f=C_{1}$ ln $\rho+C_{2}$. It is easily verified that the boundary condition $\underline{n} \times \nabla f=0$ is fulfilled. This f-function leads to the familiar form of $\underline{E}$ and $\underline{H}$ in coaxial line.

There is also an infinite number of higher-mode solutions for the coaxial 1ine. These involve the Bessel's functions of the $1 \mathrm{st}$ and 2 nd kinds, and the results are somewhat more complicated than in the case of circular guide. Discussions are given in a number of texts.

\subsection{General properties of the Eigenvalue Problems}

The first two examples illustrate some of the known general properties of eigenvalue problems for finite regions (with boundaries that are sufficiently regular in the mathematical sense). For our purposes the most important properties are in fact general properties. Some of the most pertinent general properties will be stated here; the statements should be understood to apply to any one ejgenvalue problem (TM or TE). 
(a) Eigenvalues are real, discrete, non-negative, of finite degeneracy, and they form an infinite, unbounded set. (A physical corollary of the unboundedness is that at any given frequency of operation, only a finite number of waveguide modes can be above cutoff.)

(b) Eigenfunctions can be chosen real. Eigenfunctions belonging to distinct eigenvalues are mutually orthogonal; degenerate eigenfunctions can be orthogonalized (these orthogonality properties will be defined and demonstrated below). The set of all eigenfunctions of a given problem possesses the property of completeness in the sense that arbitrary functions (subject to mathematical restrictions) can be expanded in series of the eigenfunctions.

(c) An important general observation that should be made concerning our eigenvalue problems is that they depend only on the geometry of the waveguide cross-section; they do not depend upon $\omega, \mu$, and $\varepsilon$.

To establish the basic orthogonality relations we need Green's 1st and 2nd theorms in a two-dimensional form. Green's first theorem states that

$$
\int_{C} u \nabla v \cdot \underline{n d C}=\int_{S}\left(\nabla u \cdot \nabla v+u \nabla^{2} v\right) d S
$$

where $u$ and $v$ are sufficiently differentiable functions defined over a region $S$ and its boundary $C$ and $\underline{n}$ is the outward unit normal vector on C. ( $S$ and $C$ may as well be understood to pertain to the waveguide being considered.) Interchanging $u$ and $v$ and subtracting the resulting equation from (1) yields 


$$
\int_{C}(u \nabla v-v \nabla u) \cdot \underline{n} d C=\int_{S}\left(u \nabla^{2} v-v \nabla^{2} u\right) d S
$$

which is the desired form of Green's 2nd theorem.

Two functions $f_{a}, f_{b}$ are said to be (mutually) orthogonal over the region $\mathrm{S}$ if

$$
\int_{S} f_{a} f_{b} d S=0
$$

The basic orthogonality theorem, as already mentioned, is that eigenfunction belonging to distinct eigenvalues (in a given problem) are orthogonal. To prove this, say for the TM $f$-functions, we let $f_{a}, f_{b}$ be eigenfunctions belonging to distinct non-zero eigenvalues $\mathrm{K}_{\mathrm{a}}^{2}, \mathrm{~K}_{\mathrm{b}}^{2}$ in the TM problem, and put $\mathrm{u}=\mathrm{f}_{\mathrm{a}}, \mathrm{v}=\mathrm{f}_{\mathrm{b}}$ in Green's second theorem. The left side vanishes because $f_{a}=f_{b}=0$ on $C$. Since $\nabla^{2} f_{a}=-K_{a}^{2} f_{a}$ and $\nabla^{2} f_{b}=-K_{b}^{2} f_{b}$, the theorem yields

$$
0=\left(k_{a}^{2}-k_{b}^{2}\right) \int_{S} f_{a} f_{b} d S
$$

whence follows (3). (It should be observed that the left side would not necessarily vanish and the argument would not go through if one of the functions were a g-function or a TEM f-function.) The proof of orthogonality for a pair of $g$-functions belonging to distinct eigenvalues is almost identical; in this case the lefthand side of (2) vanishes because $\underline{\mathrm{n}} \cdot \nabla \mathrm{g}=0$ on $\mathrm{C}$.

For degenerate eigenfunctions the above proof fails and the functions may in fact be non-orthogonal. However, an orthogonal set can always be constructed. To illustrate, suppose that $f_{a}$, $f_{b}$ are linearly independent, real eigenfunctions belonging to the eigenvalue $\mathrm{K}^{2}=\mathrm{K}_{\mathrm{a}}^{2}=\mathrm{K}_{\mathrm{b}}^{2}$. Any linear combination 


$$
f_{1}=c_{a} f_{a}+c_{b} f_{b}
$$

is also an eigenfunction belonging to $\mathrm{K}^{2}$. We can choose $\mathrm{C}_{\mathrm{a}}$ and $c_{b}$ so that $f_{1}$ and $f_{a}$ (say) will be orthogonal. We require

$$
\int_{S} f_{1} f_{a} d S=0
$$

and this will hold provided merely

$$
c_{a}=-C_{b} \frac{\int_{S} f_{a} f_{b} d S}{\int_{S}\left(f_{a}\right)^{2} d S} .
$$

Then $f_{1}$ and $f_{a}$ form an orthogonal pair belonging to $K^{2}$.

The process of orthogonalization can be extended to the case of $\mathrm{n}$-fold degeneracy.

In what follows it will be assumed that the TM $f$-functions are all real and mutually orthogonal; similarly for the g-functions. The implications of these orthogonality relations for the electromagnetic fields $\underline{E}, \underline{H}$ of the waveguide modes will be brought out later. 


\section{DEFINITIONS OF $v$ AND i FOR WAVEGUIDE MODES}

\subsection{General}

We have outlined essentially all the basic waveguide theory that we need. Next, as an essential preliminary to setting up a "transducer" or descriptive theory of waveguide junctions, we must define and consider " $v$ " and "i" for waveguide modes. The letters $v$ and $i$ are used advisedly, for the quantities represented will be closely related to, and generalizations of, voltage and current as conventionally defined for transmission 1 ines.

The present formulation is based directly on the general description of waveguide modes furnished by (2.3-1) and (2.4-1). For our purpose it is sufficient as well as necessary to consider the transverse components of the fields, and so the $z$-components will be ignored. Considering a TM-mode, say, we observe that $\nabla f$ and $\nabla f \times \underline{e}_{z}$, which are two-component vector functions of coordinates in the transverse plane, determine the patterns of $\underline{E}$ and $H$ in the transverse plane. If the arbitrary factor implicit in $f$ is fixed, we obtain what we may call basis (or unit) field pattern functions. The scalar coefficients in the equations then specify the "complex amplitudes" of the actual fields relative to the basis fields. We can regard this as a division of the field expressions into quantitative and qualitative parts. The question of the disposition of numerical factors between the two kinds of parts necessarily arises. This is essentially a question of normalization, and the choice of normalization constants is essentially a matter of convenjence. 
A method of carrying out the above ideas might by initiated straightforwardly in the following manner. Considering a TM mode, mode "a", say, one might define $\underline{e}_{a}^{\circ}=C_{1} \nabla f_{a}, \underline{h}_{-a}^{\circ}=C_{2} \underline{e}_{z} \times \nabla f_{a}$ and write

$$
\underline{E}_{t}=v_{a} \underline{e}_{a}^{0}, \underline{H}_{t}=i_{a} \underline{h}_{a}^{0}
$$

where $\underline{E}_{t}$ and $\underline{H}_{t}$ are the transverse components of $\underline{E}, \underline{H}$ in $(2.3-1)$. (then by comparison with $(2.3-1)$, one would have $\left.v_{a}=\phi_{a}^{\prime} / C_{1}, i_{a}=i \omega \varepsilon \phi_{a} / C_{2}\right)$. These definitions, if adopted, would provide the desired and important features that the quantity $\operatorname{Re}\left(v_{\mathrm{a}} \overline{\mathrm{i}}_{\mathrm{a}}\right)$ be proportional to the energy flux along the waveguide in mode " $a$ " and that the quantity $v_{a} / i_{a}$ appear in the role of modal impedance. From these properties it would follow that the combinations $\mathrm{C}_{1} \overline{\mathrm{C}}_{2}$ and $\mathrm{C}_{2} / \mathrm{C}_{1}$ would be more significant than $\mathrm{C}_{1}$ and $\mathrm{C}_{2}$ themselves and could be regarded as new normaizing constants. This is recognized in the procedure for setting up basis fields to be adopted here: the arbitrary constant implicit in $f_{a}$ (or $g_{a}$ ) is used to normalize $\underline{e}_{\mathrm{a}}^{\circ} \times \underline{h}_{\mathrm{a}}^{\circ}$, and a separate constant is introduced to normalize $\mathrm{e}_{\mathrm{a}}^{\circ}$ and $\underline{\mathrm{h}}_{\mathrm{a}}^{\circ}$ relative to each other.

\subsection{Basic Definjtions and Relations}

The ideas discussed in the preceding two paragraphs are formalized as follows. For any one mode, mode "a", the transverse components of $\underline{E}$, $\underline{H}$ may be written

$$
\begin{aligned}
& \underline{E}_{\mathrm{t}}=\mathrm{v}_{\mathrm{a}} \mathrm{e}_{\mathrm{a}}^{0}, \\
& \underline{\mathrm{H}}_{\mathrm{t}}=\mathrm{i}_{\mathrm{a}}-_{\mathrm{a}}^{\circ},
\end{aligned}
$$


where

$$
\underline{\mathrm{e}}_{\mathrm{a}}^{\circ}= \begin{cases}\nabla \mathrm{f}_{\mathrm{a}} & \text { for TM }- \text { TEM modes } \\ \nabla \mathrm{g}_{\mathrm{a}} \times \underline{\mathrm{e}}_{\mathrm{z}} \text { for TE modes }\end{cases}
$$

and in a 11 cases

$$
\underline{h}_{\mathrm{a}}^{\circ}=\zeta_{\mathrm{a}}^{\circ} \eta_{\mathrm{a}} \underline{\mathrm{e}}_{\mathrm{z}} \times \underline{\mathrm{e}}_{\mathrm{a}}^{\circ}
$$

where $\zeta_{\mathrm{a}}^{\circ}$ is a normalization constant and $\eta_{\mathrm{a}}$ is the "wave admittance" of the mode involved [for TM modes $\eta_{a}=\omega \varepsilon / \gamma_{a}$ (reducing to $\sqrt{\varepsilon / \mu}$ for TEM modes), and for TE modes $\left.n_{a}=\gamma_{a} /(\omega \mu)\right]$. We require that $\mathrm{e}_{-}^{\circ}$ be real (in accordance with the previous statement that $f$, $g$ may be chosen real). Although $\zeta_{\mathrm{a}}^{\circ}$ is essentially arbitrary, it is convenient to at once introduce the restriction that $\zeta_{a}^{\circ}$ shall be chosen so that the product $\zeta^{\circ} n_{a}$ will be a real positive number. Then, by (3), $\underline{\mathrm{h}}_{\mathrm{a}}^{\circ}$ will be real. If we use the term "amplitude" somewhat loosely, we can say that fixing $\zeta_{a}^{\circ}$ fixes the ratio of the amplitudes of $\underline{e}_{-}^{\circ}$ and $\underline{h}_{a}^{\circ}$; the product of the amplitudes of $\underline{\mathrm{e}}_{\mathrm{a}}^{\circ}$ and $\underline{h}_{\mathrm{a}}^{\circ}$ may be fixed by the requirement that

$$
\frac{1}{2} \int_{S} \underline{e}_{\mathrm{a}}^{\circ} \times \underline{h}_{\mathrm{a}}^{\circ} \cdot \underline{\mathrm{e}}_{\mathrm{z}}^{\mathrm{d}} \mathrm{dS}=\mathrm{w}_{\mathrm{a}}^{\circ}
$$

where $w_{a}^{\circ}$ is a normalization constant, which, in view of the conditions laid down thus far, must be real and positive. It will be seen that (2), (3), and (4), plus the specification of a sign, suffice to define $\underline{\mathrm{e}}_{\mathrm{a}}^{\circ}$ and $\underline{\mathrm{h}}_{\mathrm{a}}^{\circ}$ uniquely in terms of the normalization constants.

Equations (1) serve to implicitly define the quantities $v_{a}$, $i_{a}$ relative to any given determination of the basis fields. 
We may now define the quantity

$$
v_{a} / i_{a}=\zeta_{a}
$$

as the impedance of the field of mode "a" in any given transverse surface $z$ = const. (For fixed excitation, $\zeta_{a}$ will be a function of $z$; for fixed $z, \zeta_{a}$ will depend upon the state of excitation and may take on any value, real or complex.) That particular value of $\zeta_{a}$ that corresponds to a field consisting solely of a wave traveling (or exponentially damped) in the $+z$-direction is termed the characteristic impedance of the mode being considered. The above formulation has been arranged so that the characteristic impedance of mode "a" is precisely the normalization constant $\zeta_{a}^{\circ}$, as we shall now verify. Using a superscript "+" to denote quantities associated with a + z-directed field, we put $\phi=A \exp (i \gamma z)$ and obtain from (2.3-1) or (2.4-1)

$$
\underline{H}_{t}^{+}=\eta_{a} \underline{e}_{z} \times \underline{E}_{t}^{+}
$$

Substitution of (1) and (3) into this equation yields

$$
v_{\mathrm{a}}^{+} / \mathrm{i}_{\mathrm{a}}^{+}=\zeta_{\mathrm{a}}^{\circ}
$$

as was to be shown.

What we may call the "complex energy flux" in the + z-direction across a surface at a given $z=$ const. is given by

$$
W=\frac{1}{2} \int_{S} \underline{E} \times \underline{\bar{H}} \cdot \underline{e}_{z} d S .
$$

According to (1-5), the corresponding time-average energy flux or power is $\operatorname{Re}(W)$. We shall evaluate (7) for the general case in which a number of modes (in general including TE, TM, and TEM 
modes) are simultancously present in the waveguide. The generalization of (1) is simply

$$
\begin{aligned}
& \underline{\mathrm{E}}_{\mathrm{t}}=\sum \mathrm{v}_{\mathrm{a}} \mathrm{e}_{\mathrm{a}}^{\circ}, \\
& \underline{\mathrm{H}}_{\mathrm{t}}=\sum \mathrm{j}_{\mathrm{a}} \underline{\mathrm{h}}_{\mathrm{a}}^{\circ},
\end{aligned}
$$

where the sums go over the modes present. It may be noted that a knowledge of the transverse components of $\underline{E}, \underline{H}$ (as given by (8)) is necessary and sufficient for the evaluation of (7). It will be convenient to use the notation

$$
\left[\underline{E}^{\prime}, \underline{H}^{\prime \prime}\right]=\frac{1}{2} \int_{S} \underline{E}^{\prime} \times \underline{H}^{\prime \prime} \cdot \underline{\mathrm{e}}_{\mathrm{Z}}^{\mathrm{d} S}
$$

where $\underline{E}^{\prime}$ and $\underline{H}^{\prime \prime}$ are not nccessarily components of the same electromagnetic field. In this notation,

$$
W=[\underline{E}, \underline{H}]=\sum_{a, b} v_{a} \bar{i}_{b}\left[\underline{e}_{a}^{\circ}, \underline{h}_{b}^{o}\right]
$$

Now, under the assumption that degenerate $f$ or $g$ functions are chosen properly, it can be assumed that $\left[\underline{e}_{\mathrm{a}}, \underline{\mathrm{h}}_{\mathrm{b}}^{\circ}\right]$ will vanish whenever $\mathrm{a}$ and $\mathrm{b}$ denote distinct modes. The orthogonality proofs, given in Appendix $A$ of this chapter, are carried out with the aid of Green's and Stokes' theorems. Nine cases are involved altogether, since $e_{-}^{\circ}$ may be associated with a TE, a TM, or a TEM mode, and independently ${ }_{-}^{\circ}$ may be associated with any one of the three types of modes. In virtue of the orthogonality and the normalization relation (4), we have

$$
\left[\underline{e}_{\mathrm{a}}^{\circ}, \underline{h}_{\mathrm{b}}^{\circ}\right]=\delta{ }_{\mathrm{ab}} \mathrm{w}_{\mathrm{a}}^{\circ},
$$


where $\delta_{a b}$ is the Kronecker delta, and so

$$
w=\sum \bar{i}_{a} w_{a}^{\circ} v_{a}
$$

If $w_{a}^{0}$ were chosen equal to $1 / 2$ for all modes, we see that power would be computed as if we had a number of pairs of terminals (one pair for each mode) in an alternating-current network, with peak values of voltage and current $v_{a}, i_{a}$ at the terminal pairs. We shall leave $w_{a}^{\circ}$ arbitrary in our general formulas, however, since we wish to be able to see the effects of various choices of $\mathrm{w}_{\mathrm{a}}^{\circ}$.

It should be clear that not only does (8) determine $\underline{E}_{t}, \underline{H}_{t}$ when $v_{a}, i_{a}$ are given but also it implicitly determines $v_{a}, i_{a}$ when $\underline{E}_{t}, \underline{H}_{t}$ are given. This implicit relation can readily be made explicit by using (11); thus

$$
\begin{aligned}
& v_{a}=\left[\underline{E}_{t}, \underline{h}_{a}^{\circ}\right] / w_{a}^{\circ} \\
& i_{a}=\left[\underline{e}_{a}^{\circ}, \underline{H}_{t}\right] / w_{a}^{\circ} .
\end{aligned}
$$

These formulas are similar to those for the calculation of coefficients in Fourier expansions.

\subsection{Some Consequences of the Basic Definitions}

Basis fields for the $T E_{20}$ mode in rectanguzar waveguide.

Using the notation previously used in the discussion of rectangular waveguide, we have for the $\mathrm{TE}_{10}$ mode $g=c \cos (\pi x / a)$, where $c$ is to be determined. (Mode indices, such as "a" or "1,0", will be omitted when not needed.) From (4.2-2, $-3,-4)$, it follows that 


$$
\begin{aligned}
& \underline{e}^{0}= \pm\left(\zeta^{\circ} n\right)^{-\frac{1}{2}} \cdot 2\left[w^{\circ} /(a b)\right]^{\frac{1}{2}} \sin (\pi x / a) \underline{e}_{y} \\
& \underline{h}^{\circ}=\mp\left(\zeta^{\circ} n\right)^{+\frac{1}{2}} \cdot 2\left[w^{\circ} \cdot(a b)\right]^{\frac{1}{2}} \sin (\pi x / a) \underline{e}_{x},
\end{aligned}
$$

where $\eta=\gamma /(\omega \mu)$. In these explicit expressions the roles of $\zeta^{\circ}$ and $\mathrm{w}^{\circ}$ are evident. It may be observed here, as in the general case, that the basis fields may be frequency-dependent. Thus, for frequencies above cutoff, $n$ may be written as $\sqrt{\varepsilon / \mu}\left[1-\left(\omega_{c} / \omega\right)^{2}\right]^{\frac{1}{2}}$, and the often-used normalizations $\zeta^{\circ}=1$, $w^{\circ}=1$ produce basis fields involving the quantity $\left[1-\left(\omega_{c} / \omega\right)^{2}\right]^{\frac{1}{4}}$ $\left(\omega_{c}\right.$ is $2 \pi$ times cut-off frequency). Frequency-independent basis fields can be constructed by choosing appropriate frequencydependent normalization "constants"; e.g., $w^{\circ}=1, \zeta^{\circ}=1 / \eta$.

Principar modes. For principal modes $\mathrm{v}_{\mathrm{a}}$ and $\mathrm{i}_{\mathrm{a}}$ can be made to coincide with voltage and current as defined in transmission-line theory. We shall illustrate this in the case of coaxial waveguide. Again, as in subsection 3.2, we use the coordinates $\rho, \theta$ and radii $a, b$, taking $b>a$. The normalizations $w^{\circ}=1 / 2$ and $\zeta^{\circ}=(2 \pi n)^{-1} \ln (b / a)$, where $n=\sqrt{\varepsilon / h}$, are appropriate (it will be recognized that $\zeta^{\circ}$ has been set equal to the conventional characteristic impedance in coaxial line). These normalizations lead to

$$
\begin{aligned}
& \underline{\mathrm{e}}^{0}=[\log (\mathrm{b} / \mathrm{a})]^{-1} \underline{\mathrm{e}}_{\rho} / \rho \\
& \underline{\mathrm{h}}^{\circ}=(2 \pi)^{-1} \underline{\mathrm{e}}_{\theta} / \rho,
\end{aligned}
$$

where $\underline{e}_{\rho}$ is the radial unit vector, $\underline{e}_{\theta} \equiv \underline{e}_{z} \times \underline{e}_{\rho}$, and a choice of sign has been made. Now, peak-value transmission-line voltage and current may be defined by the line integrals 


$$
V=\int_{L_{1}} E \cdot d \underline{l}, \quad I=\int_{L_{2}} \underline{H} \cdot d \underline{l},
$$

where $L_{1}$ is a path from the inner conductor to the outer, $L_{2}$ is a loop encircling the inner conductor (in the general direction of $\left.\underline{e}_{\theta}\right)$, and both paths are restricted to lie in a given transverse surface. Under this restriction these line integrals are independent of the particular paths (a consequence of the fact that $\underline{E}$, $\underline{H}$ for TEM modes are derived from solutions of a potential problem). If we now combine (2) and (3), we obtain

$$
\begin{aligned}
& V=v \int_{L_{1}} \underline{e}^{\circ} \cdot d \underline{l}=v, \\
& I=i \int_{L_{2}} \underline{h}^{\circ} \cdot d \underline{l}=i,
\end{aligned}
$$

where the right-hand equations result from the evaluation of the line integrals. We may note that (4) implies equality in units and dimensions as we11 as numerical equality.

Dependence of $v_{a}$, $i_{a}$ upon $z$. The waveguide quantities $v_{a}$, $i_{a}$, as functions of the coordinate $z$, obey equations of transmission line form. For a TM-TEM mode we compare (4.2-1) with $(2.3-1)$ and find

$$
v_{a}=\phi^{\prime}, \quad i_{a}=i \omega \varepsilon \phi /\left(\zeta_{a}^{\circ} n_{a}\right)
$$

for a TE mode we similarly find

$$
v_{a}=i \omega \mu \phi, \quad i_{a}=\phi^{\prime} /\left(\zeta_{a}^{\circ} \eta_{a}\right) .
$$

From either pair of equations we obtain after differentiating,

$$
\begin{aligned}
& \frac{d v a}{d z}=i_{\gamma_{a}} \zeta_{a}^{\circ}, \\
& \frac{d i a}{d z}=i\left(\gamma_{a} / \zeta_{a}^{\circ}\right) v_{a},
\end{aligned}
$$


using (2.2-2) and the definitions of the wave-admittance in (4.2-3). Equations (5) are the differential equations of a transmission line having series impedance $z_{\ell}=-i \gamma_{a} \zeta_{a}^{0}$ per unit length and shunt admittance $Y_{\ell}=-i \gamma_{a} / \zeta_{a}^{\circ}$ per unit length. According to transmission-line theory such a line would have propagation constant

$$
\gamma_{\ell}=\sqrt{Y_{\ell} Z_{l}}=i \gamma_{a}
$$

and characteristic impedance

$$
\zeta_{\ell}^{0}=\sqrt{Z_{\ell} T_{l}^{-}}=\zeta_{\mathrm{a}}^{0}
$$

--just the values pertaining to the actual problem, which may or may not be a transmission-line problem in the narrow sense.

It is clearly very useful to know that the waveguide $v_{a}$, $i_{a}$ for any mode do satisfy equations of transmission-line form, since it means that a considerable body of transmission-line theory is jmmediately applicable to wavegujde problems.

Dimensional analysis. In the present discussion we use the customary symbol. [A] to denote "the dimensions of A." From (1) we can conveniently extract the dimensional formulas

$$
\begin{aligned}
& {\left[\underline{\mathrm{e}}^{0}\right]=\left[\zeta^{\circ} n\right]^{-\frac{1}{2}}\left[\mathrm{w}^{\circ}\right]^{\frac{1}{2}}[\text { meter }]^{-1},} \\
& {\left[\underline{h}^{\circ}\right]=\left[\zeta^{\circ} n\right]^{+\frac{1}{2}}\left[\mathrm{w}^{\circ}\right]^{\frac{1}{2}}[\text { meter }]^{-1},}
\end{aligned}
$$

which are, of course, independent of the particular mode and waveguide considered in (1). (Note: The unit vectors $\underline{e}_{x}$ satisfy $\underline{e}_{x} \cdot \underline{e}_{-x}=1$ independent of the primary units and so are dimension10ss.] Since in the MKS system [E] $=$ [volts/meter] and [II] $=$ [amperes/meter $]$, it follows from (4.2-1) and (6) that 


$$
\begin{aligned}
& {[v]=[\operatorname{volts}]\left[\zeta^{\circ} n\right]^{+\frac{1}{2}}\left[w^{\circ}\right]^{-\frac{1}{2}},} \\
& {[i]=[\text { amperes }]\left[\zeta^{\circ} n\right]^{-\frac{1}{2}}\left[w^{\circ}\right]^{-\frac{1}{2}} .}
\end{aligned}
$$

With the additional datum $[n]=[\mathrm{ohm}]^{-1}$ these formulas enable one to calculate the dimensions and units of $v$ and $i$ for any choice of the dimensions and units of $\zeta^{\circ}$ and $w^{\circ}$. Several examples are

TABLE 2. Schemes of units and dimensions.

$\left.\begin{array}{llll}\text { Scheme No. } & {\left[\mathrm{w}^{\circ}\right]} & {\left[\zeta^{\circ}\right]} & {[\mathrm{v}]}\end{array}\right]$

\begin{tabular}{ccccc} 
& $*$ & ohms & volts & amperes \\
\hline 2 & watts & $*$ & $*$ & $*$ \\
\hline 3 & $*$ & $*$ & (watts) $^{\frac{1}{2}}$ & (watts) $^{\frac{1}{2}}$ \\
\hline
\end{tabular}

Note: (a) $*$ = dimensionless

(b) Variables a and $\mathrm{b}$, defined below in subsection 5.3, are dimensionally the same as $v$. The occurrence of the dimension [watt] $]^{\frac{1}{2}}$ does not imply that our a's and b's represent so-called power waves that have been introduced in the recent literature.

shown in Table 2. Scheme No. 1 was actually employed in the discussion of $v$ and $i$ for the principal mode in coax, and it emphasizes the close relation that exists between $\zeta^{\circ}, v$, and $i$ and the corresponding conventional quantities. Elements of all three schemes may be identified, at least partially, in the literature. 


\section{IMPEDANCE, ADMITTANCE, AND SCATTLRING \\ MATRICES FOR WAVEGUIDE JUNCTIONS}

\subsection{Genera1}

For the present purposes a waveguide junction is a linear electromagnetic system possessing ideal waveguide leads (which individually may be of arbitrary cross-section). Unless otherwise stated, a waveguide junction will be considered to be a passive system subject to excitation solely through the effects of nonattenuated waveguide modes in the waveguide leads. (Attenuatedmode interactions are excluded here mainly because they are usually avoided in practice.)

\subsection{Definition of Impedance and Admittance Matrices}

In the $m^{\text {th }}$ of the $n$ waveguide leads of a given waveguide junction we choose a transverse, cross-sectional "terminal surface" and denote it $\mathrm{S}_{\mathrm{m}}$. From section 4, we know that the transverse components of $\underline{E}$, $\underline{H}$ on $S_{m}$ can be expressed in the form

$$
\begin{aligned}
I_{m t}=\sum_{a=1}^{\nu_{m}} v_{m a-m a}, & (m=1,2, \ldots, n) \\
H_{m t} & =\sum_{a=1}^{\nu_{m}} i_{m a-m a}{ }_{m}^{\circ},
\end{aligned}
$$

where $\nu_{m}$ is the number of propagated modes supported in the $\mathrm{m}^{\text {th }}$ waveguide and $\mathrm{e}_{-\mathrm{ll}}^{\circ}, \underline{h}_{\mathrm{ma}}^{\circ}$ are real basis fields subject to (a) the "impedance normalization" 


$$
{ }_{-\mathrm{ma}}^{\circ}=\zeta_{\mathrm{ma}}^{\circ} n_{\mathrm{ma}}-\mathrm{n} \times \mathrm{e}_{\mathrm{ma}}^{\circ}
$$

and (b) the "power normalization" and orthogonality relations

$$
\frac{1}{2} \int_{S_{m}} e_{-m a}^{\circ} \times \underline{h}_{m b}^{\circ} \cdot \underline{n}_{m} d S=\delta_{a b} w_{m a}^{\circ}
$$

where ${ }_{-m}$ denotes the unit vector on $S_{m}$ directed into the junction, $n_{\mathrm{ma}}$ is the wave admittance of mode "a" in waveguide $\mathrm{m}$, and $\zeta_{\mathrm{ma}}^{\circ}$, $\mathrm{w}_{\mathrm{ma}}^{\circ}$ are real positive normalization constants.

In view of their association with the field on a terminal surface, the quantities $v_{m a}$, $i_{m a}$ in ( $I$ ) are often called terminal variables.

The total number of propagated modes involved will be denoted by $\mathrm{N}$ :

$$
N=\sum_{m=1}^{n} \nu_{m}
$$

and, incidentally, the waveguide junction may be called an "Nport." (Note that $\mathrm{N}$ is the number of pairs of terminal variables, not necessarily the number of separate waveguide leads. This usage appears to be firmly established.)

It will be convenient at a number of points in what follows to employ a single subscript to identify a given mode in a given waveguide; this presumes merely that the entire set of modes involved has been indexed in some definite manner. For immediate use we define the column matrices 


$$
v=\left(\begin{array}{c}
v_{1} \\
v_{2} \\
\vdots \\
v_{N}
\end{array}\right), \quad i=\left(\begin{array}{c}
i_{1} \\
i_{2} \\
\vdots \\
i_{N}
\end{array}\right) .
$$

The matrix $v$ determines and is determined by $\underline{E}_{t}$ on all the terminal surfaces; similarly, i determines and is determined by $\underline{H}_{t}$ on all the terminal surfaces. Now, on the basis of certain existence and uniqueness theorems of electromagnetic theory, but ignoring all mathematical refinements, we can say that the specification of either $\underline{E}_{t}$ or $\underline{H}_{t}$ on all terminal surfaces is just sufficient to determine $\underline{E}$ and $\underline{H}$ throughout the junctions. Thus if $\mathrm{V}$ is given, $\underline{\mathrm{E}}, \underline{\mathrm{H}}$, and $\mathrm{i}$ are determined. Since the system being considered is linear and passive, the existence of a linear, homogeneous relation connecting $v$ and $i$ is implied. In fact, we may write

$$
v=Z i, \text { or } i=Y v \text {, }
$$

thereby defining the impedance matrix $Z$ and the admittance matrix Y. These matrices are mutually inverse, for the relation

$$
v=Z Y V
$$

which follows directly from (5), holds for arbitrary $v$ and thus implies ${ }^{2}$

\footnotetext{
${ }^{2}$ In this discussion it is tacitly assumed that $\mathrm{v}$ and $i$ may be prescribed arbitraxily (but not simultaneously!) and that $Z$ and $Y$ both exist. In the "strictly realizable" cases (defined in subsection 5.4) these assumptions are justified by theorems proved in an appendix to this chapter, subsection 7.3. In other cases the assumed conditions may or may not hold, and the linear relationship that must exist anong the terminal variables may have to be stated in some form other than the ones assumed above.
} 


$$
Z Y=1
$$

where "l" (being used in a matrix equation) denotes the unit matrix of dimension $\mathrm{N}$.

It is important to realize that the elements $z_{p q}$ of $z$ are analogous to the open-circuit input and transfer impedances of network theory; similarly, the $\mathrm{Y}_{\mathrm{pq}}$ are analogous to the shortcircuit input and transfer admittances of network theory.

\subsection{The Scattering Matrix}

We define the quantities $a_{p}, b_{p}$ in terms of $v_{p}, i_{p}$ by means of the equations

$$
\begin{aligned}
& a_{p}+b_{p}=v_{p} \\
& a_{p}-b_{p}=\zeta_{p}^{\circ} i_{p}
\end{aligned}
$$

for $p=1,2, \ldots, N$. If $b_{p}=0$, then

$$
v_{p} / i_{p}=\zeta_{p}^{\circ}
$$

hence, from the meaning of characteristic impedance, $a_{p}$ is the amplitude (relative to $\underline{e}_{p}^{0}$ ) of the electric field of the incident progressive component of mode $p$ at its terminal surface $S_{m(p)}$. Similarly, $b_{p}$ can be seen to be the amplitude of the electric field of the emergent progressive component of mode $p$ at $S_{m(p)}$. Let

$$
\begin{aligned}
& a+b=v, \\
& a-b=\zeta_{0} i
\end{aligned}
$$


be the matrix form of (1); here $\zeta_{0}$ is the diagonal matrix of characteristic impedances,

$$
\zeta_{0}=\left(\begin{array}{llll}
\zeta_{1}^{\circ} & 0 & \ldots & 0 \\
0 & \zeta_{2}^{\circ} & & 0 \\
& & & \\
\ldots & \ldots & \ldots & \ldots \\
0 & 0 & \zeta_{N}^{\circ}
\end{array}\right) .
$$

The scattering matrix represents the transformation from a set of waves incident on a given waveguide junction to the corresponding set of waves emergent from the junction. We write this transformation as

$$
\mathrm{b}=\mathrm{Sa},
$$

thereby defining the scattering matrix $S$. The relations connecting $Z, Y$, and $S$ are derivable by elementary matrix algebra and may be written

$$
\begin{aligned}
& Z=\frac{1+S}{1-S} \zeta_{O}=Y^{-1}, \\
& \frac{Z \zeta_{O}^{-1}-1}{Z \zeta_{O}^{-1}+1}=S=\frac{1-\zeta_{O} Y}{1+\zeta_{O} Y} .
\end{aligned}
$$

The "rational fractions" written here have unambiguous meaning because of the commutation property exemplified by $(1+S)(1-S)^{-1}=(1-S)^{-1}(1+S)$. This is a property of the form of the expressions independent of any special properties of $Z, Y$, and $S$. 


\subsection{Power Relations}

General formulas. The total complex power input to the waveguide junction is, from (5.2-1) and (5.2-3),

$$
W=\frac{1}{2} \sum_{m=1}^{n} \int_{S_{m}} \underline{E} \times \underline{\bar{H}} \cdot \underline{n}_{m} \mathrm{dS}=\sum_{m=1}^{\mathrm{n}} \sum_{a=1}^{\nu_{m}} \overline{\mathrm{i}}_{\mathrm{ma}} w_{m a}^{\circ} v_{m a} \cdot
$$

Or, using the simplified indexing,

$$
W=\sum_{p=1}^{N} \bar{i}_{p} w_{p}^{o} v_{p}
$$

In matrix notation this becomes

$$
W=i *_{0} v
$$

where the "*" denotes the Hermitian conjugate and $w_{0}$ is the diagonal matrix of the power normalization constants. The additional expressions

$$
W=i * W_{0} Z i=v * Y * W_{0} v
$$

follow from (5.2-5) with the aid of the general matrix relation $(A B)^{*}=B^{*} A^{*}$; also, using $(5.3-2,-3)$ and taking the real part,

$$
\operatorname{Re}(W)=a *\left(w_{0} \zeta_{0}^{-1}-S * w_{0} \zeta_{0}^{-1} S\right) a,
$$

where (in accordance with the prescriptions in subsections 4.2 and 5.2) the diagonal elements of the diagonal matrix $w_{0} \zeta_{0}^{-1}$ are real and positive since attenuated modes have been excluded. Realizability conditions. The condition that the junction be passive means that $Z, Y$, and $S$ must be such that $\operatorname{Re}(W) \geq 0$ for arbitrary excitation. The conditions thus placed on Z, Y, and $S$ may be said to be "realizability" conditions for passive junctions. 
To illustrate the mathematical expression of these conditions, we consider the scattering matrix S. In (3) we set $\mathrm{w}_{\mathrm{O}} \zeta_{\mathrm{O}}^{-1}-\mathrm{S}^{*} \mathrm{~W}_{\mathrm{O}} \zeta_{\mathrm{O}}^{-1} \mathrm{~S}=\mathrm{H}_{\mathrm{S}}$ and note that $\mathrm{H}_{\mathrm{S}}$ is Hermitian. Since we have

$$
\operatorname{Re}(W) \equiv{ }^{*} H_{S} \mathrm{a}
$$

conditions on $\operatorname{Re}(W)$ are equivalent to conditions on the matrix of the Hermitian form a*H $\mathrm{S}^{\mathrm{a}}$. We now distinguish three cases of realizability, according to whether the dissipation of the junction is positive for every non-zero a, for only some a, or for no a. (Of course the last two conditions are "realizable" only in the technical sense in which we are using the term; a term such as "passivity" might seem more appropriate, but "realizability" has the sanction of usage.)

(a) "Strict realizability": $\operatorname{Re}(W)>0$ for every non-zero a. In this case, the Hermitian matrix $\mathrm{H}_{S}$ and the associated form are said to be "positive definite" (or, sometimes, "strictly positive"). A useful criterion for this case is: a Hermitian matrix is positive definite if and only if all its principal minors ${ }^{3}$ are positive [2].

(b) "Semi-realizabịity": $\operatorname{Re}(W) \geq 0$ for every a and $\operatorname{Re}(W)=0$ for some non-zero a. In this case $H_{S}$ and the associated form are said to be "positive semi-definite"; a criterion for this case is: a Hermitian matrix is positive semi-definite if and only if it is singular and all its principal minors are nonnegative [2].

\footnotetext{
${ }^{3} \mathrm{~A}$ "principal minor" of a matrix $\mathrm{A}$ is a minor whose diagonal is a part of the diagonal of $A$. Thus a principal minor is obtained by selecting rows and columns with the same sets of indices. Special cases of the principal minors of $\mathrm{A}$ are the diagonal elements of $\mathrm{A}$ and the determinant of $\mathrm{A}$.
} 
(c) "Losslessness": $\operatorname{Re}(W)=0$ for every a. For this case it is easily shown directly that $\mathrm{H}_{\mathrm{S}}$ must be the $(\mathrm{N} \times \mathrm{N})$ zero matrix.

For the impedance and admittance matrices $Z$ and $Y$, the Hermitian matrices corresponding to $\mathrm{H}_{\mathrm{S}}$ are $\mathrm{H}_{Z}=\left(\mathrm{w}_{0} Z+Z{ }^{*_{W_{0}}}\right) / 2$ and $\mathrm{H}_{\mathrm{Y}}=\left(\mathrm{w}_{\mathrm{O}} \mathrm{Y}+\mathrm{Y}^{*} \mathrm{~W}_{\mathrm{O}}\right) / 2$, respectively. Realizability conditions for $Z$, Y, and $S$ may be summarized as follows: The matrices $\mathrm{H}_{Z}$, $\mathrm{H}_{\mathrm{Y}}$, and $\mathrm{H}_{\mathrm{S}}$ must be positive definite, positive semi-definite, or zero, according as case (a), (b), or (c) applies.

At this point it may well be observed that certain simplifications may be obtained in the above analysis and results by suitable choices of normalization. For example, if $w_{0}$ is a scalar matrix (i.e., a scalar multiple of the unit matrix), it cancels out in the statement of realizability conditions for $Z$ and $Y$; similarly if $w_{0} \zeta_{0}^{-1}$ is a scalar matrix, it cancels out in the statements pertaining to $S$ (See Table 3 ). In the lossless case, $Z$ and $Y$ become anti-Hermitian, and $S$ becomes unitary.

\subsection{Reciprocity}

Provided that the constitutive parameters $\underline{\mu}, \underline{\underline{\varepsilon}}$ (which may be complex) are symmetric tensors (which may reduce to scalars) it can be shown that [3]

$$
\sum_{m=1}^{n} \int_{S_{m}}\left(\underline{E}^{\prime} \times \underline{H}^{\prime \prime}-\underline{E}^{\prime \prime} \times \underline{H}^{\prime}\right) \cdot \underline{\hat{n}}_{m} \mathrm{dS}=0,
$$

where $\underline{E}^{\prime}, \underline{H}^{\prime}$, and $\underline{E}^{\prime \prime}, \underline{H}^{\prime \prime}$ are any two electromagnetic fields (of the same frequency) that can exist in the given waveguide junction. 
This is a basic expression of reciprocity for waveguide junctions, and we shall use it to find the manifestation of reciprocity in Z, Y, and S. From (5.2-1) and (5.2-3) it follows that ( 1 ) is equivalent to

$$
\tilde{i} " w_{0} v '-\tilde{i} w_{0} v " \equiv 0 \text {, }
$$

where the $\sim$ denotes the transpose of a matrix.

To find the consequences of (2) for $Z$ and $Y$, we first insert $v^{\prime}=Z i^{\prime}, v^{\prime \prime}=Z i^{\prime \prime}$. After taking the transpose of the second term, we obtain

$$
\tilde{i}^{\prime \prime}\left(w_{0} z-\tilde{z}_{w_{0}}\right) i^{\prime} \equiv 0 \text {, }
$$

which implies

$$
w_{0} z-\tilde{Z}_{0}=0
$$

since $i^{\prime}, i^{\prime \prime}$ are arbitrary. Since $Z=Y^{-1}$ (and $\tilde{Z}=\tilde{Y}^{-1}$ ), the relation

$$
w_{O} Y-\tilde{Y}_{W_{O}}=0
$$

is an immediate consequence of ( $3 a)$.

To find the conditions imposed on $S$ we use (5.3-2) and find from (2)

$$
\tilde{a}^{\prime \prime} \zeta_{0}^{-1} w_{0} b^{\prime}-\tilde{a}^{\prime} \zeta_{0}^{-1} w_{0} b^{\prime \prime} \equiv 0
$$

Hence, using $\mathrm{b}=\mathrm{Sa}$, we must have

$$
\zeta_{O}^{-1} w_{O} S-\tilde{S} \zeta_{O}^{-1} w_{O}=0
$$


The statement of the reciprocity conditions may be simplified by appropriate choice of normalizations, and it happens that the appropriate choices are the same as in the case of the realizability conditions considered above. Table 3 furnishes a summary of all these relations in simplified form.

TABLE 3

Realizability and reciprocity conditions under simplifying normalizations

\begin{tabular}{cccc}
\hline & $Z$ & $Y$ & $S$ \\
& $\left(w_{0}\right.$ scalar, $\zeta_{0}$ & $\left(w_{0}\right.$ scalar, $\zeta_{0}$ & $\left(w_{0} \zeta_{0}^{-1}\right.$ scalar $)$ \\
arbitrary) & arbitrary & \\
Realizability: & $Z+Z^{*}$ PD, & $Y+Y^{*}$ PD, & $1-S^{*} S$ PD, \\
& PSD, or 0. & PSD, or 0. & PSD, or 0. \\
Reciprocity: & $\tilde{Z}=Z$ & $\tilde{Y}=Y$ & $\tilde{S}=S$ \\
\hline
\end{tabular}

Note:
(a) $\mathrm{PD}=$ positive definite, $\mathrm{PSD}=$ positive semi-definite.
(b) Reciprocity may, of course, hold simul- taneously with any case of realizability.

\subsection{Change of Basis}

In the preceding two subsections we have already noted some of the effects of impedance and power re-normalization. In this subsection we shall merely mention the main ideas underlying these and other "changes of basis." We may regard 
all the equations in this section thus far (with the exception of (5.5-1), for which the question of basis is irrelevant), as having been set up for some particular set of normalization constants; we recognize that these equations could equally well have been set up for some other set of normalization constants; and we may inquire how the quantities $v, i, z$, etc., change--or transform-- when the normalization is changed. These transformations must be such that basis-dependent equations are invariant in form and such that the physical quantities being described are invariant absolutely [4].

A rather different and more interesting type of change of basis should be mentioned. This permits one to take new terminal variables that are linear combinations of two or more of the old ones. Matters can be arranged so that neither impedance nor power normalization is affected [5].

\subsection{Sources; Joining Equations}

General. We have already noted that our basic expression for the power input contributed by one waveguide mode, $\mathrm{w}_{\mathrm{a}}=\frac{1}{2} \overline{\mathrm{i}}_{\mathrm{a}} \mathrm{v}_{\mathrm{a}}$ (for $\mathrm{w}_{\mathrm{a}}^{\circ}=\frac{1}{2}$ ), is of the same form as the expression for input power at a pair of terminals in an alternatingcurrent network. In this sub-section we shall consider two further fundamental relations that are required to establish the basis of the application of equations of the form of network equations to waveguide problems. 
Sources. For simplicity we consider a waveguide "junction" having just one waveguide lead, in which just one mode propagates. We choose a terminal surface and consider the terminal variables $v_{1}, i_{1}$. We assume that the junction is linear (from an external point of view) and not necessarily passive. Now, the most general linear relation connecting $v_{1}$ and $i_{1}$ can be written

$$
v_{1}=z_{g 1} i_{1}+v_{g 1}
$$

or

$$
i_{1}=Y_{g 1} v_{1}+i_{g 1},
$$

where $Z_{g 1}, Y_{g l}, v_{g l}, i_{g l}$ are constants, and to avoid mathematical details, we assume $Z_{g 1} \neq 0, Y_{g l} \neq 0$. The presence of sources may be manifested in these equations in two ways: in the inhomogeneity of the equations $\left(v_{g l} \neq 0, i_{g l} \neq 0\right)$ or in a violation of the realizability condition as applied to $Z_{g l}$ and $Y_{g 1}$. The latter possibility means for these $1 \times 1$ matrices $\operatorname{Re}\left(\mathrm{Z}_{\mathrm{g} I}\right)<0$ and $\operatorname{Re}\left(\mathrm{Y}_{\mathrm{g} 1}\right)<0$, as may be seen from Table 3 . It should be observed that (la) and (lb) respectively represent Thévenin's and Norton's theorems.

Alternatively, we may describe our source in terms of the terminal variables $b_{1}, a_{1}$. The most general linear relation connecting these variables may be written

$$
\mathrm{b}_{1}=\mathrm{s}_{\mathrm{g} 1} \mathrm{a}_{1}+\mathrm{b}_{\mathrm{g} 1}
$$


where $\mathrm{S}_{\mathrm{gl}}$ and $\mathrm{b}_{\mathrm{gl}}$ are constants characterizing the source. The equation states that the general emergent wave $b_{1}$ is the sum of the wave $b_{g 1}$ that would be emitted into a nonreflecting load and the reflected portion of the incident wave $a_{1}$. From Table 3 we see that violation of the realizability condition for the $1 \times 1$ scattering "matrix" $S_{g 1}$ means $\left|S_{g 1}\right|>1$.

Joining equations. We consider that a waveguide lead of one system is to be connected to a waveguide lead of another system. We assume that the terminal surfaces associated with each system have been so located that they coincide when the connection is made (See Fig. 2). (It is also assumed that no electrical discontinuity exists after the connection is made; we are not considering waveguide discontinuity problems.) The transverse components of $\underline{E}, \underline{H}$ on the common terminal surface $S$ are then given by the equations

$$
\begin{aligned}
& \underline{E}_{t}=\sum v_{a} \underline{e}_{a}^{0} \\
& \underline{H}_{t}=\sum i_{a} \underline{h}_{a}^{\circ}
\end{aligned}
$$

associated with the one system and also by the equations

$$
\begin{aligned}
& \underline{E}_{t}^{\prime}=\sum v_{\mathrm{a}}^{\prime} \underline{\mathrm{e}}_{\mathrm{a}}^{\circ} \\
& \underline{\mathrm{H}}_{\mathrm{t}}^{\prime}=\sum \mathrm{i}{ }_{\mathrm{a}}^{\prime} \mathrm{h}_{\mathrm{a}}^{\circ}
\end{aligned}
$$




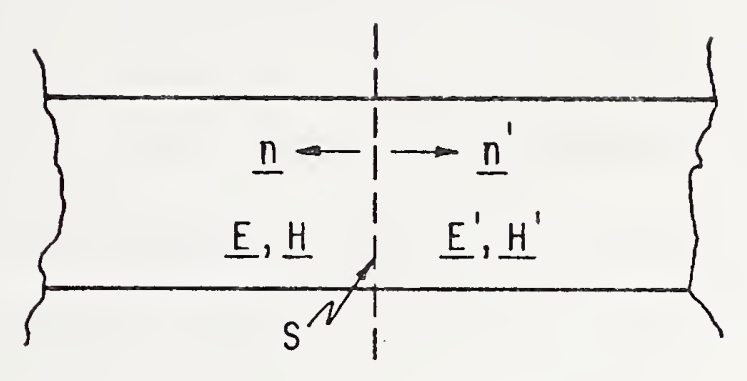

Fig. 2. Coincident terminal surfaces.

associated with the other. We may and do assume

$$
\underline{\mathrm{e}}_{\mathrm{a}}^{0}=\underline{\mathrm{e}}_{\mathrm{a}}^{0}
$$

this implies

$$
\underline{h}_{\mathrm{a}}^{0}=-\underline{h}_{\mathrm{a}}^{0}
$$

since, by $(5.2-2), \underline{n}=-\underline{n} '$. Now, for the electromagnetir.

fields corresponding to (3) to be continued properly across

$S$ it is necessary and sufficient that $\underline{E}_{t} \equiv \underline{E}_{t}^{\prime}, \underline{H}_{t} \equiv_{t}^{\prime}$.

This means that it is in turn necessary and sufficient that

$$
\begin{aligned}
& v_{a}=v_{a}^{\prime} \\
& i_{a}=-i_{a}^{\prime}
\end{aligned}
$$

for each mode involved. We call these equations joining equations. They are of exactly the same form as the equations in circuit theory that describe the joining of two pairs of terminals. To verify this, consider that the terminal pairs shown in Fig. 3 are to be joined. With the sign conventions indicated in the figure, circuit theory obviously requires 
$v=v^{\prime}, i=-i^{\prime}$. (The sign conventions are consistent with the restrictions imposed by (4) together with the choice of $\underline{n}_{m}$ as the inward normal on $S_{m}$ in $(5.2-2)$ and $\left.(5.2-3).\right)$

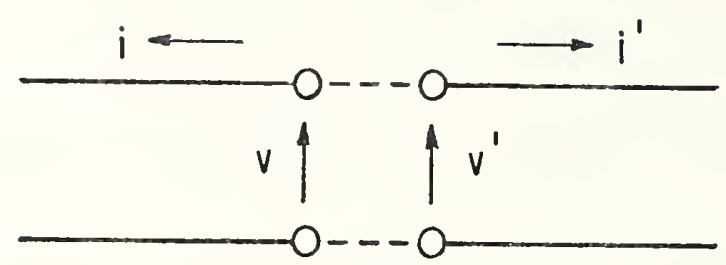

Fig. 3. An "equivalent circuit" for joining.

In the equations that characterize a waveguide junction, such as the matrix equation $v=Z i$, the number of variables is twice the number of equations. This statement applies also to $(5.3-3),(1)$, and (2). We can say that in a waveguide junction characterized by a set of $\mathrm{N}$ equations, the electromagnetic state has $\mathrm{N}$ degrees of freedom. However, if loads or sources ${ }^{4}$ are connected at all terminal surfaces and the joining equations are applied, the number of equations become equal to the number of unknowns in the system. Thus (except for special cases where the equations are not all independent) the terminal variables and hence the electromagnetic state become determinate.

${ }^{4}$ A passive waveguide junction possessing just one waveguide lead (multimode or not) is termed a "load" or a termination"; if not passive, it is termed a "source." 


\section{NETWORK EQUATIONS}

\subsection{Genera1}

We conclude this chapter with the presentation of a number of basic network equations, which will be directly useful later, and which will provide immediate (though limited) illustration of the content of the discussion thus far. For the present purpose, the use of the wave-amplitudes $a, b$, and the scattering-matrix scheme is appropriate. We shall take the power normalization constants $w_{m a}^{\circ}$ all equal to $1 / 2$, thus securing peak (rather than, e.g., root-meansquare) values for the variables $a$ and $b$. This is convenient because it agrees with the customary use of peak values for field quantities $\underline{E}$ and $\underline{H}$, which will be encountered in greater detail later. Characteristic impedances we leave arbitrary, except that we naturally assume equal characteristic impedances when applying the joining equations. Retainiag an explicit symbol for characteristic impedance (or for the corresponding admittance) is, in the first place, dimensionally convenient. The flexibility thus provided will be available for the eventual study of transient or frequencydomain response of antennas, which may be simplified by suitable choice of frequency-dependent characteristic impedances (subsection 4.3). It will be convenient (and in agreement with later usage) to use characteristic admittance $n_{0}=1 / \zeta^{\circ}$, instead of using $\zeta^{\circ} \operatorname{explicitly.~}$ 


\subsection{Source and Load}

To start with a simple and important case, we suppose that a one-port load is to be connected to a one-port source (or "generator") and that we wish to find the power delivered to the load (Fig. 4). The load and the source are character-

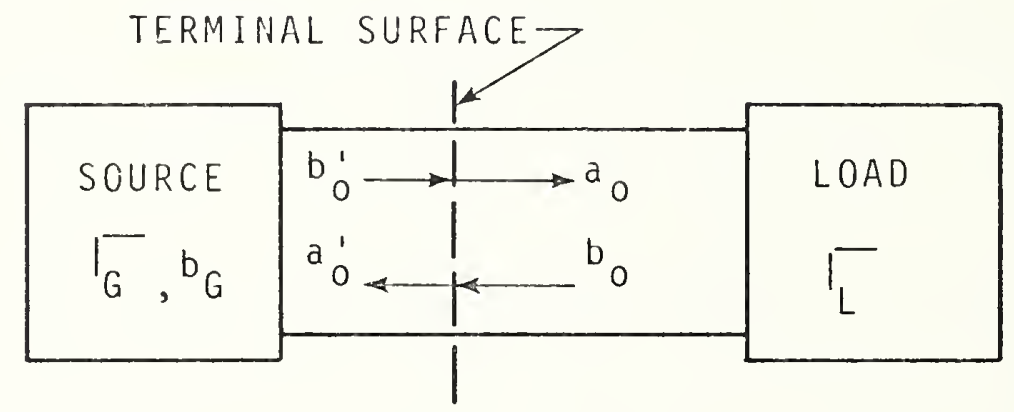

Fig. 4. Source and load.

ized by the equations

$$
b_{0}=\Gamma_{L_{0}} a_{0}, \quad b_{0}^{\prime}=\Gamma_{G} a_{0}^{\prime}+b_{G} \cdot
$$

The equation on the left is a one-dimensional version of (5.3-3), and the one on the right comes directly from (5.7-2). [We adhere to the common convention of denoting reflection coefficients of one-ports (or equivalent one-ports) by I's.] The joining equations are

$$
b_{0}^{\prime}=a_{0}, \quad a_{0}^{\prime}=b_{0}
$$

as follows from (5.7-5) and (5.3-1) (and as might be expected). Solving the system of (1) and (2) for $b_{0}^{1}$ or $a_{0}$, we obtain 


$$
a_{0}=\frac{b_{G}}{1-\Gamma_{G} \Gamma_{L}}
$$

The expression in the denominator is of a type characteristic of scattering equations: it can be interpreted in terms of a summation of waves multiply reflected between the source and the load.

From (5.4-2), applied in the present case and under the present conventions, the net (time-average) power delivered to the load is

$$
\mathrm{P}_{\mathrm{L}}=\frac{1}{2} \eta_{\mathrm{O}}\left|\mathrm{a}_{\mathrm{O}}\right|^{2}\left(1-\left|\Gamma_{L}\right|^{2}\right)=\frac{1}{2} \eta_{O}\left|\mathrm{~b}_{\mathrm{G}}\right|^{2} \frac{1-\left|\Gamma_{L}\right|^{2}}{\left|1-\Gamma_{G} \Gamma_{L}\right|^{2}} .
$$

If, in particular, the load is a conjugate match to the generator $\left(\Gamma_{L}=\bar{\Gamma}_{G}\right)$, then

$$
\mathrm{P}_{\mathrm{L}}=\mathrm{P}_{\mathrm{A}}=\frac{1}{2} n_{\mathrm{O}}\left|\mathrm{b}_{\mathrm{G}}\right|^{2} \frac{1}{1-\left|\Gamma_{\mathrm{G}}\right|^{2}} \text {, }
$$

where $\mathrm{P}_{\mathrm{A}}$ is termed the available power of the generator and is the maximum power that can be delivered by the given generator to a passive load. (Maximum power is not delivered to a reflectionless load unless the generator itself is reflectionless.) The delivered power can be expressed in terms of the available power and a mismatch factor in the following ways: 


$$
\begin{aligned}
P_{L} & =P_{A} \frac{\left(1-\left|\Gamma_{G}\right|^{2}\right)\left(1-\left|\Gamma_{L}\right|^{2}\right)}{\left|1-\Gamma_{G} \Gamma_{L}\right|^{2}} \\
& =P_{A}\left(1-\left|\frac{\Gamma_{L}-\bar{\Gamma}_{G}}{1-\Gamma_{G} \Gamma_{L}}\right|^{2}\right) .
\end{aligned}
$$

This result recurs frequently, usually in the form of (6a). The second form shows clearly the maximum property of $\mathrm{P}_{\mathrm{A}}$.

6.3 Reciprocity, Realizability, and Losslessness for 2-Ports If a 2 -port is reciprocal, then, under the normalization conventions adopted, the general reciprocity constraint given in $(5.5-4)$ requires merely that

$$
n_{01} S_{12}=n_{02} S_{21}
$$

The familiar result $S_{12}=S_{21}$ is obtained when the characteristic admittances are chosen equal.

Under the normalization conventions adopted, the "loss matrix" $\mathrm{H}_{\mathrm{S}}$ of (5.4-3) applied to 2-ports, becomes

$$
H_{S}=\frac{1}{2}\left(\begin{array}{ll}
n_{01}\left(1-\left|S_{11}\right|^{2}\right)-n_{02}\left|s_{21}\right|^{2} & -n_{01} \bar{s}_{11} s_{12}-n_{02} \bar{S}_{21} s_{22} \\
-n_{01} S_{11} \bar{S}_{12}-n_{02} s_{21} \bar{S}_{22} & n_{02}\left(1-\left|s_{22}\right|^{2}\right)-n_{01}\left|s_{12}\right|^{2}
\end{array}\right) \text {. }
$$

Strict realizability requires 


$$
\begin{aligned}
& 0<\frac{n_{02}}{n_{01}} \frac{\left|s_{21}\right|^{2}}{1-\left|s_{11}\right|^{2}}<1 \\
& 0<\frac{n_{01}}{n_{02}} \frac{\left|s_{12}\right|^{2}}{1-\left|s_{22}\right|^{2}}<1
\end{aligned}
$$

and

$$
0<\operatorname{det}\left(\mathrm{H}_{\mathrm{S}}\right)
$$

The three conditions are necessary; it is easy to see that the third together with either one of the first two is sufficient. Physical interpretations of these equations will be noted below.

In Appendix $B$ it is shown that the characteristic numbers (or eigenvalues) of a strictly realizable scattering matrix must all be less than unity in magnitude. Because of the relative complexity of (2), it is well to realize that this result is available.

Losslessness requires the expressions in (2a), (2b), and (2c) to be equal to 1,1 , and 0 , respectively. The first two conditions are

$$
\begin{aligned}
& \frac{n_{02}}{n_{01}} \frac{\left|s_{21}\right|^{2}}{1-\left|s_{11}\right|^{2}}=1, \\
& \frac{n_{01}}{n_{02}} \frac{\left|s_{12}\right|^{2}}{1-\left|s_{22}\right|^{2}}=1,
\end{aligned}
$$


and the third condition reduces to

$$
n_{01} \bar{S}_{11} S_{12}+n_{02} \bar{S}_{21} S_{22}=0
$$

- - a weighted orthogonality. In this case the three necessary conditions are also sufficient. If $\eta_{01}=\eta_{02}$, the familiar result that $S$ must be unitary is obtained, and an alternate set of necessary and sufficient conditions for a lossless 2 -port can be given as

$$
\bar{S}_{11}=S_{22} / D, \quad \bar{S}_{21}=-S_{12} / D, \quad|D|=1,
$$

where $D=\operatorname{det} S$.

6.4 Two-Port Network Equations

We consider a 2 -port connected between a source and a load (Fig. 5) and give an annotated series of equations, which should prove handy for reference.

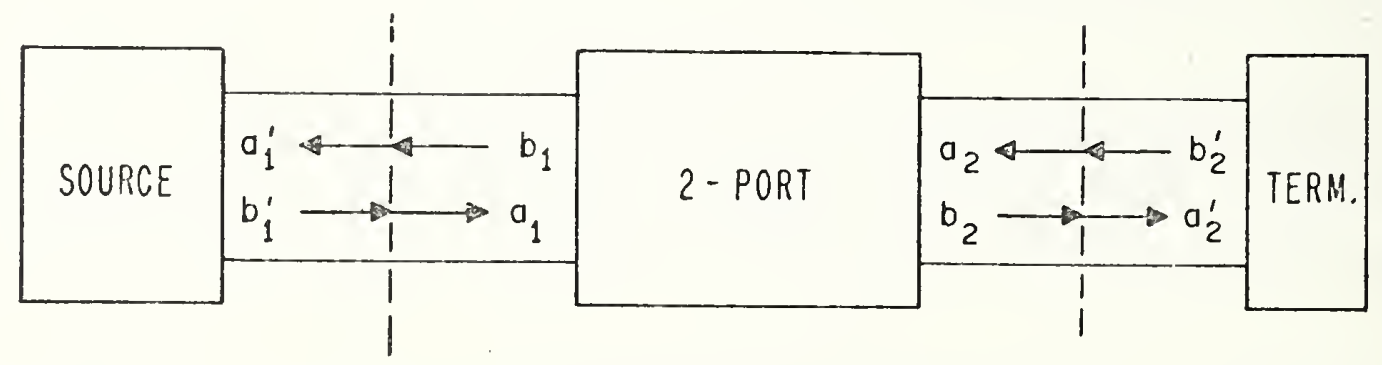

Fig. 5. Two-port connecting source and load.

The scattering equations for the 2 -port are written

$$
\begin{aligned}
& b_{1}=s_{11} a_{1}+s_{12} a_{2}, \\
& b_{2}=s_{21} a_{1}+s_{22} a_{2},
\end{aligned}
$$


and the load has reflection coefficient $\Gamma_{L}$, so that we have $a_{2}=\Gamma_{L} b_{2}$.

We first obtain the elementary but important equation

$$
\frac{\mathrm{b}_{2}}{\mathrm{a}_{1}}=\frac{\mathrm{s}_{21}}{1-\Gamma_{L} \mathrm{~s}_{22}}
$$

The occurrence of ratios of this type is a characteristic of the type of antenna measurements to be described. The quantity represented can be regarded as an "effective" value of $\mathrm{S}_{21}$ as modified by a "reflection factor" $\left(1-\Gamma_{L} S_{22}\right)^{-1}$. Next, using the last result and (1a), we find for the reflection coefficient $\Gamma_{1}$ presented by the loaded 2 -port,

$$
\begin{aligned}
\Gamma_{1} \equiv \frac{b_{1}}{a_{1}} & =S_{11}+\frac{S_{12} S_{21} \Gamma_{L}}{1-\Gamma_{L} S_{22}} \\
& =\frac{S_{11}-\Gamma_{L} \text { det } S}{1-\Gamma_{L} S_{22}},
\end{aligned}
$$

where det $S$ is the determinant of $S$.

In (3), the 2 -port can be regarded as producing a transformation of the load reflection coefficient, and the 2 -port may be present for the purpose of producing a desired transformation. In this case the 2 -port may be called a tuner, which is represented ideally and analytically by a lossless, reciprocal, adjustable 2-port. It can be shown that any $\Gamma_{L}$ inside the unit circle in the complex plane can be transformed to any other value inside rhe unit circle by such a tuner. The use of a variable length of lossless line and a variable shunt susceptance to transform a given $\Gamma_{L}$ to 0 is a frequently discussed instance of this theorem [6]. 
The efficiency of a 2-port is defined as the ratio of the net power $\mathrm{P}_{\mathrm{L}}$ delivered to the load to the net power input $\mathrm{P}_{1}$ to the 2-port. We form this ratio,

$$
\varepsilon=\frac{P_{L}}{P_{1}}=\frac{n_{02}\left|b_{2}\right|^{2}\left(1-\left|\Gamma_{L}\right|^{2}\right)}{n_{01}\left|a_{1}\right|^{2}\left(1-\left|\Gamma_{1}\right|^{2}\right)}
$$

and use (2) and (3) to obtain

$$
\varepsilon=\frac{n_{02}}{n_{01}} \cdot \frac{\left|S_{21}\right|^{2}\left(1-\left|\Gamma_{L}\right|^{2}\right)}{\left|1-\Gamma_{L} S_{22}\right|^{2}-\left|S_{11}-\Gamma_{L} \operatorname{det} S\right|^{2}}, \quad(6.4-4)
$$

It should be noted--perhaps emphasized--that this quantity is wholly independent of the characteristics of the source that may be attached. Furthermore, if a tuner (or any other lossless 2-port) is inserted between the source and the given 2 -port, the efficiency of the combination of the two 2 -ports still has the value given by (4). This follows directly from the losslessness of the interposed 2-port and is true even though power transfer may be increased (or decreased) by insertion and adjustment of the tuner.

Clearly, if a 2-port is lossless, its efficiency must be unity; and if it is strictly realizable, its efficiency must be less than unity.

If the load is non-reflecting, (4) becomes

$$
\varepsilon=\frac{n_{02}}{n_{01}} \frac{\left|s_{21}\right|^{2}}{\left(1-\frac{\left.\left.s_{11}\right|^{2}\right)}{}\right.}
$$


(In this special case the properties stated in the preceding paragraph follow directly from $(6.3-2,-3)$.$) Equation (5) is$ similar to expressions for power gain of antennas that will be obtained later. The antenna gain expression is not identically equal to unity, even for an antenna free of ohmic power loss, but it does possess the property of invariance with respect to the insertion or adjustment of a lossless tuner. Insufficient appreciation of this invariance property has been a source of confusion in discussions of antenna gain measurements.

We next calculate net power delivered to the load. For this we need $b_{2}$; with the aid of $(6.2-3),(2)$, and (3) we obtain

$$
\mathrm{b}_{2}=\mathrm{b}_{\mathrm{G}} \mathrm{S}_{21} / \mathrm{D}
$$

where $\mathrm{D} \equiv\left(1-\Gamma_{G} S_{11}\right)\left(1-\Gamma_{L} S_{22}\right)-\Gamma_{G} \Gamma_{L} S_{21} S_{12}$. Hence

$$
\mathrm{P}_{1}=\frac{\dot{1}}{2} n_{02}\left|\mathrm{~b}_{\mathrm{G}} \mathrm{S}_{21}\right|^{2}\left(1-\left|\Gamma_{\mathrm{L}}\right|^{2}\right) /|\mathrm{D}|^{2} \cdot(6.4-7)
$$

This quantity evidently depends in general upon all parameters of source, 2-port, and load involved.

The power delivered by a given 2-port connected between a given source and a given load may be described analytically or determined experimentally by comparison with the power delivered by another (known or specified) reference 2-port connected between the same generator and load. If $\mathrm{P}_{\mathrm{L}}^{\prime}$ is the net power delivered by the reference 2 -port and $P_{L}$ is the net power delivered with the 2 -port of interest substituted for 
the reference 2-port, the ratio $\mathrm{L}_{\mathrm{S}}=\mathrm{P}_{\mathrm{L}}^{\prime} / \mathrm{P}_{\mathrm{L}}$ is called the substitution loss ratio (and is frequently expressed in dB). This is the general concept of substitution loss. If the reference 2port amounts to a direct connection, $\mathrm{P}_{L}^{\prime}$ is given by $(6.2-4)$, and the corresponding $\mathrm{L}_{S}$ is called the insertion loss ratio; if the reference 2 -port is a tuner providing a conjugate match between source and load, $\mathrm{P}_{\mathrm{L}}^{\prime}$ is given by $(6.2-5)$, and $\mathrm{L}_{\mathrm{S}}$ is called the transducer power-loss ratio. Expressions for these and other loss ratios may be derived, as needed, from formulas given above. Given: Two 2-ports, described by the scattering equations

$$
\begin{aligned}
& \mathrm{b}_{1}=\mathrm{s}_{11} \mathrm{a}_{1}+\mathrm{s}_{12} \mathrm{a}_{2}, \\
& \mathrm{~b}_{2}=\mathrm{s}_{21} \mathrm{a}_{1}+\mathrm{s}_{22} \mathrm{a}_{2}
\end{aligned}
$$

and

$$
\begin{aligned}
& \mathrm{b}_{1}^{\prime}=\mathrm{T}_{11} \mathrm{a}_{1}^{\prime}+\mathrm{T}_{12} \mathrm{a}_{2}^{\prime}, \\
& \mathrm{b}_{2}^{\prime}=\mathrm{T}_{21} \mathrm{a}_{1}^{\prime}+\mathrm{T}_{22} \mathrm{a}_{2}^{\prime}
\end{aligned}
$$

connected so that $b_{2}^{\prime}=a_{1}$ and $a_{2}^{\prime}=b_{1}$, as indicated in Fig. 6 .

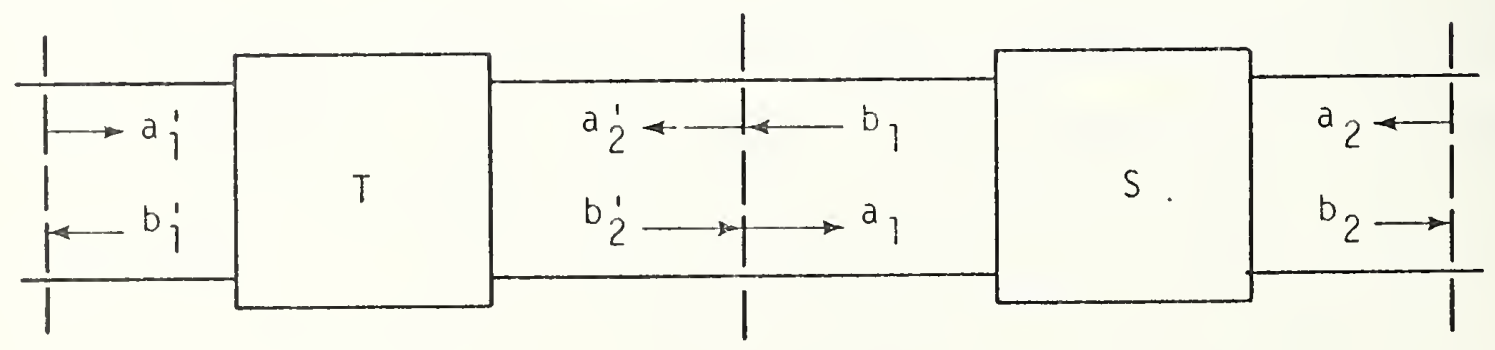

Fig. 6. Coupled 2-ports. 
To find: The scattering matrix of the composite 2-port. (To treat this type of problem more systematically, one would use cascading or transfer matrices instead of the scattering matrices.) Eliminating the intermediate variables, we obtain

$$
\begin{aligned}
& \mathrm{b}_{1}^{\prime}=\left(\mathrm{T}_{11}+\frac{\mathrm{T}_{12} \mathrm{~T}_{21} \mathrm{~S}_{11}}{1-\mathrm{S}_{11} \mathrm{~T}_{22}}\right) \mathrm{a}_{1}^{\prime}+\frac{\mathrm{T}_{12} \mathrm{~S}_{12}}{1-\mathrm{S}_{11} \mathrm{~T}_{22}} \mathrm{a}_{2} \\
& \mathrm{~b}_{2}=\frac{\mathrm{T}_{21} \mathrm{~S}_{21}}{1-\mathrm{S}_{11} \mathrm{~T}_{22}} \mathrm{a}_{1}^{\prime}+\left(\frac{\mathrm{T}_{22} \mathrm{~S}_{21} \mathrm{~S}_{12}}{1-\mathrm{S}_{11} \mathrm{~T}_{22}}+\mathrm{S}_{22}\right) \mathrm{a}_{2}
\end{aligned}
$$

A simple and useful example of this result is obtained if we take $T$ to represent the properties of a single mode in a section of uniform lossless waveguide of length $\ell$. Then

$$
T=\left(\begin{array}{cc}
0 & e^{i \beta \ell} \\
e^{i \beta \ell} & 0
\end{array}\right),
$$

where $B$ is the appropriate phase constant, and the composite matrix becomes

$$
\left(\begin{array}{ll}
s_{11} e^{2 i \beta l} & s_{12} e^{i \beta l} \\
s_{21} e^{i \beta l} & s_{22}
\end{array}\right)
$$

This also expresses the effect of a translation of the "lefthand" terminal surface of the 2 -port represented by $S$. In this application $\&$ may be either positive or negative. 


\subsection{Discrete-Mode Model of Coupled Antennas}

We may obtain a realistic discrete-mode model of two coupled antennas by imagining (or considering) them to be mounted inside a large, infinitely long, perfectly conducting waveguide, as suggested in Fig. 7. The realism is such that a complete formulation would anticipate much of that given in subsequent chapters for the free-space case. Here we shall be more brief. Where appropriate, notation will be chosen to anticipate that used later.

\section{LARGE ENCLOSING WAVEGUIDE-}

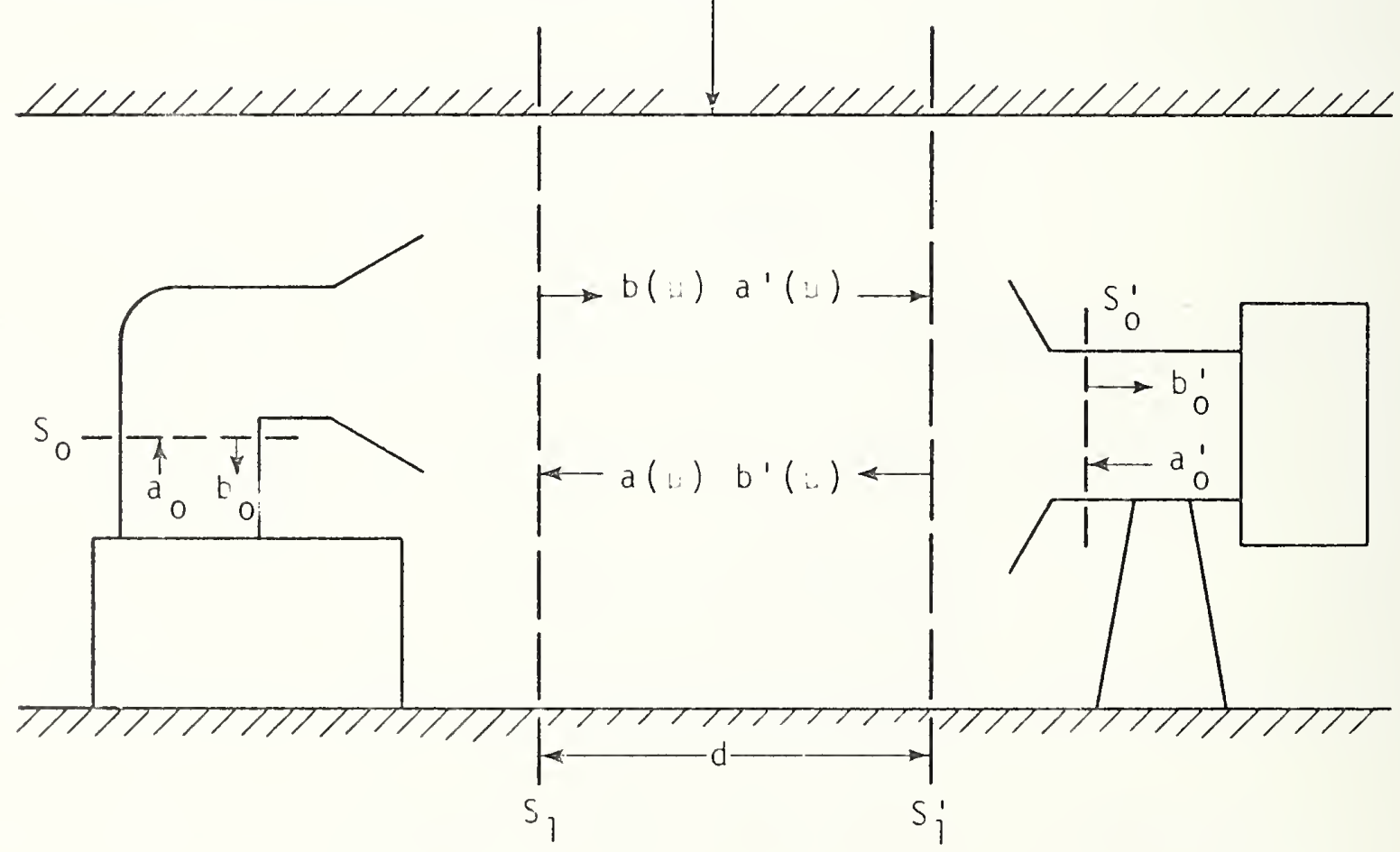

Fig. 7. Discrete-mode model of coupled antennas.

Although primarily intended as an introduction to the antenna scattering-matrix descriptions formulated later, in 
algebra are required, the discrete-mode model may have heuristic or analytical value of its own. The larger the enclosing waveguide, the more exact will be the discrete-mode model. This suggests a formulation of the real antenna problem by a limiting process. The is not recommended as a method of derivation, but is discussed below for the insight it may provide. (See end of this subsection.) The concept involved has been used in several analytical and numerical studies $[7,8]$.

The scattering matrix for each antenna is defined with the antenna in place in the enclosing waveguide, independent of the other antenna (or in the absence of the other antenna). For the antenna on the left in Fig. 7 we write

$$
\begin{gathered}
\mathrm{b}_{0}=\mathrm{S}_{000^{\mathrm{a}}}+\sum_{\mu^{\prime}=1}^{\mathrm{N}} \mathrm{S}_{01}(\mu) \mathrm{a}(\mu) \\
\mathrm{b}(\mu)=\mathrm{S}_{10}(\mu) \mathrm{a}_{0}+\sum_{\mu^{\prime}=1}^{N} \mathrm{~S}_{11}\left(\mu, \mu^{\prime}\right) \mathrm{a}\left(\mu^{\prime}\right)
\end{gathered}
$$

Here the subscripts " 0 " and " 1 " refer to the terminal surfaces $\mathrm{S}_{0}$ in the antenna feed and $\mathrm{S}_{1}$ in the large waveguide; we have assumed one propagated mode in the feed, $N$ propagated modes in the large waveguide; and $\mu$ is an index for the modes in the large waveguide. For simplicity, we do not here consider the possibility of significant higher-mode interaction between antennas.

The above description is complete for the present purposes: there are to be no sources to the left of the antenna, and 
whatever radiation to the left occurs is manifested in the loss characteristics of the scattering matrix as defined. For some purposes one might need to consider incident and emergent modes on an additional terminal surface to the left of the structure. This is done later for the "real" antenna problem .

We next introduce the $\mathrm{N}$-dimensional column matrices

$$
\hat{\mathrm{b}}=\left(\begin{array}{c}
\mathrm{b}(1) \\
\vdots \\
\mathrm{b}(\mathrm{N})
\end{array}\right), \quad \hat{\mathrm{a}}=\left(\begin{array}{c}
\mathrm{a}(1) \\
\vdots \\
\mathrm{a}(\mathrm{N})
\end{array}\right),
$$

and write (1) in the partitioned matrix form

$$
\left(\begin{array}{l}
\mathrm{b}_{0} \\
\hat{\mathrm{b}}
\end{array}\right)=\left(\begin{array}{ll}
\mathrm{s}_{00} & \hat{\mathrm{s}}_{01} \\
\hat{\mathrm{s}}_{10} & \hat{\mathrm{s}}_{11}
\end{array}\right)\left(\begin{array}{l}
\mathrm{a}_{0} \\
\hat{\mathrm{a}}
\end{array}\right),
$$

or equivalently,

$$
\begin{aligned}
b_{0} & =S_{00} a_{0}+\hat{S}_{01} \hat{a}, \\
b & =\hat{S}_{10} a_{0}+\hat{S}_{11} \hat{a} .
\end{aligned}
$$

Here the $1 \times 1$ "matrix". $S_{00}$ is the antenna feed reflection coefficient; the $\mathrm{N}$-dimensional row matrix $\hat{\mathrm{S}}_{01}$ has the elements $\mathrm{S}_{01}(\mu)$ and represents the receiving characteristic of the antenna; the $\mathrm{N}$-dimensional column matrix $\hat{\mathrm{S}}_{10}$ has the elements $\mathrm{S}_{10}(\mu)$ and represents the transmitting characteristic; and, finally, the $N \times N$ matrix $\hat{S}_{11}$ with elements $S_{11}\left(\mu, \mu^{\prime}\right)$ describes the "back-scattering" of waves incident from the right. 
Sec. 6.5]

The scattering matrix for the antenna on the right is defined similarly. The scattering equaions are written

$$
\begin{aligned}
& \mathrm{b}_{0}^{\prime}=\mathrm{S}_{00}^{\prime} \mathrm{a}_{0}^{\prime}+\hat{\mathrm{S}}_{01}^{\prime} \hat{\mathrm{a}}^{\prime}, \\
& \hat{\mathrm{b}}^{\prime}=\hat{\mathrm{S}}_{10}^{\prime} \mathrm{a}_{0}^{\prime}+\hat{\mathrm{S}}_{11}^{\prime} \hat{\mathrm{a}}^{\prime}
\end{aligned}
$$

and are defined with respect to the terminal surfaces $S_{0}^{\prime}$ and $S_{1}^{\prime}$. Primes are used here (and later) to distinguish quantities associated with the antenna on the right in a transmission system.

Let the distance between the terminal surfaces $S_{1}$ and $S_{1}^{\prime}$ in the enclosing waveguide be $d$, and let the propagation constant for mode $\mu$ in this waveguide be denoted $\gamma_{\mu}$. Then the characteristics of the enclosing waveguide and joining ("modematching") are accounted for in the following equations.

$$
\begin{aligned}
a^{\prime}(\mu) & =b(\mu) \exp \left(i \gamma_{\mu} d\right), \\
a(\mu) & =b^{\prime}(\mu) \exp \left(i \gamma_{\mu} d\right) .
\end{aligned}
$$

To express these equations in matrix form, we define the diagonal matrix

$$
\hat{\mathrm{T}}=\left(\begin{array}{ccc}
\exp \left(i \gamma_{1} \mathrm{~d}\right) & \cdots & 0 \\
0 & \cdot & \exp \left(i \gamma_{N} \mathrm{~d}\right)
\end{array}\right)
$$

and thus obtain

$$
\hat{a}^{\prime}=\hat{T} \hat{b}, \quad \hat{a}=\hat{T} \hat{b}^{\prime}
$$


Cone may observe that the matrix $\left(\begin{array}{ll}0 & \hat{T} \\ \hat{T} & 0\end{array}\right)$ is the multimode generalization of the special matrix $\mathrm{T}$ in (6.4-11).)

We can now obtain complete solutions for the behavior of the system under consideration. That is, for any given sources and loads on the terminals at $S_{0}$ and at $S_{0}^{\prime}$, we can determine any of the variables, $b_{0}, a_{0}, \hat{b}$, etc. In particular, we can obtain the scattering matrix of the system 2-port, which is defined by the equations

$$
\left.\begin{array}{l}
b_{0}=M_{11} a_{0}+M_{12} a_{0}^{\prime} \\
b_{0}^{\prime}=M_{21} a_{0}+M_{22} a_{0}^{\prime}
\end{array}\right\}
$$

These equations are formally the same, and the procedure for calculation of the matrix elements is just the same, as the equations and procedure used later in the "real" antenna coupling problem in section 2 of Chapter II. For example

$$
\begin{aligned}
& M_{11}=S_{00}+\hat{S}_{01} \hat{R}^{\prime}\left(1-\hat{S}_{11} \hat{R}^{\prime}\right)^{-1} \hat{S}_{10}, \\
& M_{21}=\hat{S}_{01}^{\prime} \hat{T}\left(1-\hat{S}_{11} \hat{R}^{\prime}\right)^{-1} \hat{S}_{10},
\end{aligned}
$$

where $\hat{\mathrm{R}}^{\prime}=\hat{\mathrm{TS}}_{11}^{\prime} \hat{\mathrm{T}}$. The derivation of these cquations is suggested as an exercise for the reader.

If the enclosing waveguide of this discussion is rectangular, of dimensions $A$ and $B$, then (according to subsection 3.2) the 
Sec. 6.5]

modal field-components will be functions of the transverse $(x, y)$ coordinates of the forms

$$
\sin k_{x} x \sin k_{y} y
$$

The boundary conditions at the waveguide walls require that $\mathrm{k}_{\mathrm{x}}$ and $k_{y}$ have only the discrete values

$$
\mathrm{k}_{\mathrm{x}}=\mathrm{m} \pi / \mathrm{A}, \quad \mathrm{k}_{\mathrm{y}}=\mathrm{n} \pi / \mathrm{B},
$$

where $m$ and $n$ are integers (which may be taken non-negative). The modal propagation constants are

$$
\begin{aligned}
\gamma(m, n) & =\sqrt{k^{2}-k_{x}^{2}-k_{y}^{2}} \\
& =\sqrt{k^{2}-(m \pi / A)^{2}-(n \pi / B)^{2}},
\end{aligned}
$$

where $k^{2}=\omega^{2} \mu \varepsilon$. For any given waveguide dimensions (and frequency), we see that $\gamma(m, n)$ must eventually become imaginary as $m$ and $n$ increase (so that there can be only a finite number of modes above cutoff); we also observe that the larger the waveguide dimensions, the smaller the steps in the variation of $k_{x}$ and $k_{y}$ with $m$ and $n$. Both of these features are general properties of waveguides of finite dimensions (cf. subsection 3.3). The second feature points to the occurrence of the continuous spectrum in the free-space case.

In the free-space case, boundary conditions present no problem (since there are no boundaries), and we may choose transverse condinates according to other considerations. For 
general purposes, it is convenient to use rectangular coordinates, and it is also convenient to use the products of exponentials $\exp \left( \pm i k_{x} x\right) \operatorname{ext}\left( \pm i k_{y} y\right)$ (rather than the products of sines and cosines in (8)) as modal functions.

The discrete-mode model illustrates and suggests important features of the free-space case. However, it is not we11adapted to the derivation or even illustration of far-field (asymptotic) properties of individual antennas or of transmission systems. These properties, which are the essence of conventional antenna theory, are given later and are derived from the properties of the continuous spectrum. 


\section{APPENDICES TO CHAPTER I}

7.1 Appendix A: Modäl Orthogonalities

The derivations of the orthogonality relations used in subsection 4.2 are outlined here. We consider the nine cases in three groups, beginning with the simplest.

In the TE vS TE one finds

$$
\left[\underline{\mathrm{e}}_{\mathrm{a}, \mathrm{TE}}^{\circ}, \underline{\mathrm{h}}_{\mathrm{b}, \mathrm{TE}}^{\circ}\right]=\frac{1}{2} \zeta_{\mathrm{b}}^{\circ} \eta_{\mathrm{b}} \int_{\mathrm{S}} \nabla \mathrm{g}_{\mathrm{a}} \cdot \nabla \mathrm{g}_{\mathrm{b}} \mathrm{dS}
$$

Using Green's first theorem, and observing the boundary conditions and the differential equation satisfied by the g-functions, we transform the integral on the right and obtain

$$
\left[\underline{\mathrm{e}}_{\mathrm{a}, \mathrm{TE}}^{\circ}, \underline{\mathrm{h}}_{\mathrm{b}}^{\circ}, \mathrm{TE}\right]=\frac{1}{2} \zeta_{\mathrm{b}}^{\circ} \eta_{\mathrm{b}} \mathrm{K}_{\mathrm{b}}^{2} \int_{\mathrm{S}} \mathrm{g}_{\mathrm{a}} \mathrm{g}_{\mathrm{b}} \mathrm{dS}
$$

Thus the orthogonality (and the normalization) of the TE basisfields is referred to that of the corresponding scalar eigenfunctions, for which the orthogonality properties are already known (subsection 3.3 ).

For the four cases of mixed TE and TM-TEM basis fields we can write

$$
\begin{aligned}
\left(\zeta_{\mathrm{b}}^{\circ} n_{\mathrm{b}}\right)^{-1}\left[\underline{\mathrm{e}}_{\left.\mathrm{a}, \mathrm{TM}-\mathrm{TEM}, \underline{\mathrm{h}}_{\mathrm{b}, \mathrm{TE}}^{\circ}\right]}\right. & =\left(\zeta_{\mathrm{a}}^{\circ} \mathrm{n}_{\mathrm{a}}\right)^{-1}\left[\underline{\mathrm{e}}_{\mathrm{b}, \mathrm{TE}}^{\circ} \underline{\mathrm{h}}_{\mathrm{a}, \mathrm{TM}-\mathrm{TEM}]}\right] \\
& =\frac{1}{2} \int_{S}\left(\nabla f_{\mathrm{a}} \times \nabla g_{\mathrm{b}}\right) \cdot \underline{\mathrm{e}}_{\mathrm{z}} \mathrm{dS} .
\end{aligned}
$$


To transform this integral we use the vector identity

$$
\nabla \times\left(g_{b} \nabla f_{a}\right)=\nabla g_{b} \times \nabla f_{a}
$$

and apply Stokes' theorem to obtain

$$
\int_{S} \nabla \times\left(g_{b} \nabla f_{a}\right) \cdot \underline{e}_{z} d S=\int_{C} g_{b} \nabla f_{a} \cdot \underline{d} d C
$$

where I is the unit tangent vector on $C$. The last integral vanishes for both TM and TEM $f$-functions, since for both, $\nabla f_{a} \cdot \underline{\tau}=0$ on $\mathrm{C}$. Thus orthogonality obtains in all these mixed cases.

In the four TM-TEM vs TM-TEM cases we obtain first

$$
\left[\underline{e}_{\mathrm{a}}^{\circ}, \mathrm{TM}-\mathrm{TEM}, \underline{\mathrm{h}}_{\mathrm{b}}^{\circ}, \mathrm{TM}-\mathrm{TEM}\right]=\frac{1}{2} \zeta_{\mathrm{b}}^{\circ} \eta_{\mathrm{b}} \int_{\mathrm{S}} \nabla \mathrm{f}_{\mathrm{a}} \cdot \nabla \mathrm{f}_{\mathrm{b}} \mathrm{dS} .
$$

We denote the integral on the right by $\mathrm{I}_{\mathrm{ab}}$; Green's first theorem gives

$$
I_{a b}=\int_{C} f_{a} \nabla f_{b} \cdot \underline{n} d C-\int_{S} f_{a} \nabla^{2} f_{b} d S .
$$

If modes "a" and "b" are both TM, we thus have

$$
\left[\underline{e}_{\mathrm{a}}^{\circ}, \mathrm{TM}, \underline{\mathrm{h}}_{\mathrm{b}, \mathrm{TM}}^{\circ}\right]=\frac{1}{2} \zeta_{\mathrm{b}}^{\circ} n_{\mathrm{b}} \mathrm{K}_{\mathrm{b}}^{2} \int_{\mathrm{S}} \mathrm{f}_{\mathrm{a}} \mathrm{f}_{\mathrm{b}} \mathrm{dS}
$$

and the orthogonality (and the normalization) of the TM basisfields is referred to that of the TM f-functions.

If "a" denotes a TM-mode and "b" a TEM, Green's theorem gives $\mathrm{I}_{\mathrm{ab}}=0$ without reference to the value of $\int_{S} f_{a} f_{b}$ dS. Similarly, if "a" denotes a TEM-mode and "b" a TM, we inter- 
change subscripts in Green's theoren and again set $I_{a b}=0$. Thus orthogonality obtains in all these mixed cases.

If modes "a" and "b" are both TEM, we cannot determine orthogonality from the general formulas. But we know that if a multiplicity of linearly independent TEM $f$-functions exists, linear combinations can be formed such that $\mathrm{I}_{\mathrm{ab}}$ will vanish whenever $\mathrm{a}$ and $\mathrm{b}$ denote distinct modes. (Note that in the TEM VS TEM case there is no apparent simple relation between $I_{a b}$ and $\left.\int_{S} f_{a} f_{b} d S.\right)$

7.2 Appendix B: Characteristic Numbers of $S$

The main purpose of this appendix is to establish the theorem that if a waveguide junction satisfies the condition of strict realizability, then the characteristic numbers (or eigenvalues) of its $S$ matrix must all be less than unity in magnitude. At the same time it is easily shown that if the waveguide junction is lossless, the characteristic numbers of $S$ must all be equal to unity in magnitude.

Strict realizability requires $\operatorname{Re}(W)>0$ for any non-trivial state of exitation of the junction; by (5.4-3) this is equivalent to

$$
a^{*}\left(w_{0} \zeta_{0}^{-1}-S^{*} w_{0} \zeta_{0}^{-1} S\right) a>0
$$

for any $a \neq 0$. Let $\lambda_{i}$ denote a characteristic number of $S$, and let $x_{i}$ denote the corresponding characteristic vector [9]. Then (by definition) $s x_{i}=\lambda_{i} x_{i}$, and putting $a=x_{i}$ in (1) yields 


$$
\left(x_{i}^{*} w_{0} \zeta_{0}^{-1} x_{i}\right)\left(1-\left|\lambda_{i}^{2}\right|\right)>0 .
$$

Since the factor in parenthesis on the left is a positive real number $\left(w_{0} \zeta_{0}^{-1}\right.$ being hermitian), it follows that $\left|\lambda_{i}\right|<1$.

In the semirealizable case, for which $\operatorname{Re}(W) \geq 0$, we can conclude from the above argument only that $\left|\lambda_{i}\right| \leq 1$.

In the lossless case, for which $\operatorname{Re}(W) \equiv 0$, the argument leads to $\left|\lambda_{j}\right|=1$.

It is interesting that these results are independent of the particular choice of the (positive) impedance and power normalization constants. We have already noted that with a scalar $\mathrm{w}_{0} \mathrm{\zeta}_{0}^{-1}$, the scattering matrix is unitary in the lossless case. Thus the third result above confirms the we11-known result that the eigenvalues of a unitary matrix are equal to unity in magnitude.

7.3 Appendix C: Existence of $S$; Existence Theorems for $Z$ and $Y$

We should note that $Z$ or $Y$ or both may fail to exist for a lossless waveguide junction (which itself exists only as an approximation). For a lossless uniform waveguide terminated by a perfectly conducting, wall at a distance $\&$ from the reference surface, we have $Z_{11}=\left(Y_{11}\right)^{-1}=-i \zeta^{\circ} \tan \beta \ell$, where $\beta$ is the phase constant: $Z_{11}$ and $Y_{11}$ fail to exist only for isolated values of length or frequency. For an "ideal transformer," defined by the equations $v_{1}=r v_{2}, i_{1}=\frac{1}{r} \dot{i}_{2}$, where $r$ is a real constant, $Z$ and $Y$ do not exist at a11. Likewise, for a "canonical minimum scattering antenna," [10], non-existence of $Z$ and $Y$ is guaranteed by the definition of the device. 
In all these cases the scattering matrix affords a suitable alternative mode of description. It is in fact reasonable to postulate the existence of $\mathrm{S}$ for any (linear) waveguide junction. (It is not implied that $S$ must be non-singular; $S$ could even be the $\mathrm{N}$-dimensional zero matrix.)

If we further make the basic assumption that the elements of the column matrix a of incident wave amplitudes can take on or can be assigned arbitrary values, then, in the case of strict realizability, we can rigorously justify $(5.2-5),(5.2-6),(5.3-4)$, and (5.3-5) of the text. We first observe that in the strictly realizable case the theorem of the preceding subsection implies that the matrices $1+S$ and $1-S$ are both non-singular, for the non-singularity of these matrices is equivalent to the statement that -1 and +1 , respectively, are not characteristic numbers of $S$ [9]. We now show that:

(a) The elements of the column matrices $v$ and $i$ can take on arbitrary values. From (5.3-2, -3), which furnish the definitions of $a, b$, and $s$, we have

$$
\begin{aligned}
v & =a+b=(1+S) a \\
\zeta_{0} i & =a-b=(1-S) a .
\end{aligned}
$$

Since $1+S$ and $1-S$ are non-singular, the arbitrariness of $v$ and $i$ follows from that of a.

(b) The matrices $Z$ and $Y$ both exist. This follows from (5.3-4), which relates $Z$ and $Y$ to $S$ and shows that $Z$ and $Y$ both exist when $1+S$ and $1-S$ are both non-singular. 
7.4 Appendix D: Formulas from Vector Analysis

$$
\begin{aligned}
& \underline{A} \cdot \underline{B}=A_{x} B_{x}+A_{y} B_{y}+A_{z} B_{z} \\
& \underline{A} \times \underline{B}=\left|\begin{array}{lll}
\underline{e}_{x} & \underline{e} & \underline{e} \\
A_{x} & A_{y} & A_{z} \\
B_{x} & B_{y} & B_{z}
\end{array}\right| \\
& \underline{A} \times(\underline{B} \times \underline{C})=\underline{B}(\underline{A} \cdot \underline{C})-\underline{C}(\underline{A} \cdot \underline{B}) \\
& \underline{A} \cdot(\underline{B} \times \underline{C})=(\underline{A} \times \underline{B}) \cdot \underline{C}=\underline{C} \cdot(\underline{A} \times \underline{B}), e t c . \\
& (\underline{A} \times \underline{B}) \cdot(\underline{C} \times \underline{D})=|\underline{A} \cdot \underline{C} \underline{A} \cdot \underline{D}| \underline{B} \underline{B} \cdot \underline{D} \mid
\end{aligned}
$$

$$
\begin{aligned}
& \nabla u=\operatorname{grad} u ; \nabla \cdot \underline{F}=\operatorname{div} \underline{F} ; \nabla \times \underline{F}=\operatorname{cur} \underline{F} \\
& \begin{aligned}
\nabla(u v)=u \nabla v+v \nabla u ; \nabla \cdot(\underline{u} \underline{A})=(\nabla u) \cdot \underline{A}+u \nabla \cdot \underline{A} \\
\nabla(\underline{A} \cdot \underline{B})=(\nabla \underline{A}) \cdot \underline{B}+(\nabla \underline{B}) \cdot \underline{A} \\
=(\underline{A} \cdot \nabla) \underline{B}+(\underline{B} \cdot \nabla) \underline{A}+\underline{A} \times(\nabla \times \underline{B})+\underline{B} \times(\nabla \times \underline{A})
\end{aligned}
\end{aligned}
$$$$
\nabla \times(u \underline{A})=(\nabla u) \times \underline{A}+u \nabla \times \underline{A}
$$$$
\nabla \cdot(\underline{A} \times \underline{B})=\underline{B} \cdot(\nabla \times \underline{A})-\underline{A} \cdot(\nabla \times \underline{B})
$$$$
\nabla \cdot(\nabla \times \underline{F})=0 ; \nabla \times(\nabla u)=0 ; \nabla \cdot(\nabla u)=\nabla^{2} u
$$$$
\nabla \times(\nabla \times \underline{F})=\nabla(\nabla \cdot \underline{F})-\nabla^{2} \underline{F}
$$$$
\nabla \times(\underline{A} \times \underline{B})=\underline{A} \nabla \cdot \underline{B}-\underline{B} \nabla \cdot \underline{A}+(\underline{B} \cdot \nabla) \underline{A}-(\underline{A} \cdot \nabla) \underline{B}
$$ 
7.5 Appendix E: Some Definitions and Rules Concerning Matrices

$\underline{\text { MATRIX }}$

A matrix is a rectangular array of quantities. For example,

$$
A=\left(\begin{array}{lll}
A_{11} & A_{12} & A_{13} \\
A_{21} & A_{22} & A_{23} \\
A_{31} & A_{32} & A_{33}
\end{array}\right) .
$$

When elements are identified by subscript indices, as in this example, the first subscript identifies the row and the second identifies the column.

EQUAL MATRICES

Each element of one matrix equals corresponding element of other matrix.

ADDITION

Add each element of lst matrix to corresponding element of 2nd matrix.

Example:

$A+B=\left(\begin{array}{ll}A_{11} & A_{12} \\ A_{21} & A_{22}\end{array}\right)+\left(\begin{array}{ll}B_{11} & B_{12} \\ B_{21} & B_{22}\end{array}\right)=\left(\begin{array}{ll}\left(A_{11}+B_{11}\right) & \left(A_{12}+B_{12}\right) \\ \left(A_{21}+B_{21}\right) & \left(A_{22}+B_{22}\right)\end{array}\right)=B+A$

MULTIPLICATION

Two matrices A, B may be "multiplied" to form a "product," $A B$, provided that they are "conformable." (For conformability, the number of columns in the first matrix must equal the number of rows in the second.) The element in the $\mathrm{m}^{\text {th }}$ row and the $\mathrm{n}^{\text {th }}$ column of the product is formed by taking the "product-sum" of 
the elements in the $m^{\text {th }}$ row of the lst matrix and the elements of the $n^{\text {th }}$ column of the 2nd matrix. Thus,

$$
(A B)_{m n}=\sum_{k} A_{m k} B_{k n} .
$$

Exainple:

$A B=\left(\begin{array}{ll}A_{11} & A_{12} \\ A_{21} & A_{22}\end{array}\right)\left(\begin{array}{ll}B_{11} & B_{12} \\ B_{21} & B_{22}\end{array}\right)=\left(\begin{array}{ll}A_{11} B_{11}+A_{12} B_{21} & A_{11} B_{12}+A_{12} B_{22} \\ A_{21} B_{11}+A_{22} B_{21} & A_{21} B_{12}+A_{22} B_{22}\end{array}\right)$.

Matrix multiplication is not in general commutative, i.e., $A B \neq B A$, in general (even if both products are possible ones); but it has the very important property of being associative, $(A B) C=A(B C)$.

MULTIPLICATION BY A SCAI,AR

Multiply each element of the matrix by the scalar.

Examp1e:

$$
\lambda A=\left(\begin{array}{ll}
\lambda A_{11} & \lambda A_{12} \\
\lambda A_{21} & \lambda A_{22}
\end{array}\right) .
$$

ZERO MATRIX

All elements are zero.

DIAGONAL MATRIX

All elements are zero except elements on principal diagonal (slanting downward from left to right).

UNIT MATRIX

Diagonal matrix with diagonal elements equal to unity. 
Sec. 7.5]

Example:

$$
I=\left(\begin{array}{lll}
1 & 0 & 0 \\
0 & 1 & 0 \\
0 & 0 & 1
\end{array}\right)
$$

SCALAR MATRIX

A scalar multiple of the unit matrix.

ADJUGATE

Matrix formed by replacing each element by its cofactor, then transposing.

Examp1e:

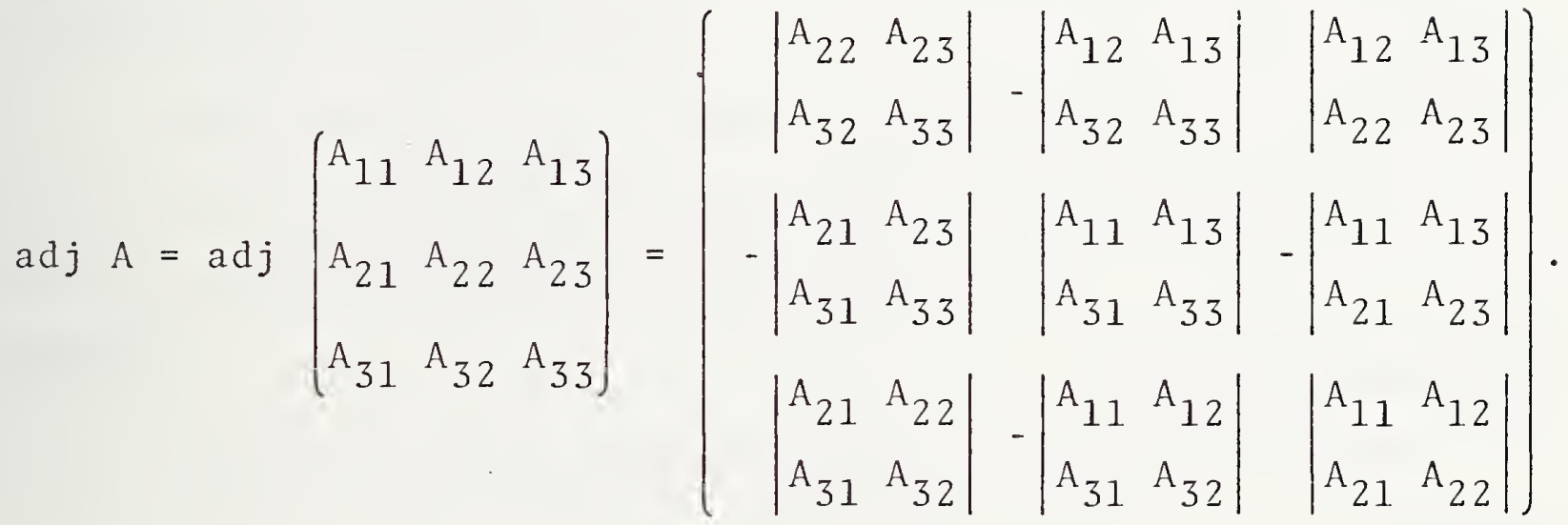

INVERSE MATRIX

If $A$ is a nonsingular matrix (i.e., if det $A \neq 0$ ), there exists a matrix $\mathrm{R}$ such that $\mathrm{RA}=\mathrm{AR}=\mathrm{I}$. The matrix $\mathrm{R}$ is termed the inverse of $\mathrm{A}$ and is denoted by $\mathrm{A}^{-1}$. The inverse is equal to the adjugate divided by the determinant of the matrix:

$$
A^{-1}=\frac{\operatorname{adj} A}{\operatorname{det} A} \quad(\operatorname{det} A \neq 0)
$$

In matrix algebra one does not speak of division by a matrix, and the notation $A / B$ is not ordinarily used (because it is ordinarily ambiguous). Instead, onc multiplies by the inverse, writing $\mathrm{B}^{-1} \mathrm{~A}$ or $\mathrm{AB}^{-1}$, whichever is wanted. 


\section{TRANSPOSE}

Matrix (denoted by tilde) formed by rearranging original matrix so that element $\Lambda_{\mathrm{mn}}$ becomes element $\tilde{\Lambda}_{\mathrm{nm}}$ (rows become columns, and vice-versa).

Example:

$$
\text { If } A=\left(\begin{array}{ll}
A_{11} & A_{12} \\
A_{21} & A_{22}
\end{array}\right), \quad \text { then } \tilde{A}=\left(\begin{array}{ll}
A_{11} & A_{21} \\
A_{12} & A_{22}
\end{array}\right) \text {. }
$$

\section{COMPLEX CONJUGATE}

Complex conjugate of each element.

Example:

$$
\text { If } A=\left(\begin{array}{ll}
A_{11} & A_{12} \\
A_{21} & A_{22}
\end{array}\right) \text {, then } \bar{A}=\left(\begin{array}{ll}
\bar{A}_{11} & \bar{A}_{12} \\
\bar{A}_{21} & \bar{A}_{22}
\end{array}\right) \text {. }
$$

HERMTTIAN CONJUGATE

Complex conjugate of transpose (denoted by asterisk). Example:

$$
\text { If } A=\left(\begin{array}{ll}
A_{11} & A_{12} \\
A_{21} & A_{22}
\end{array}\right) \text {, then } A^{*}=\left(\begin{array}{ll}
\bar{A}_{11} & \bar{A}_{21} \\
\bar{A}_{12} & \bar{A}_{22}
\end{array}\right) \text {. }
$$

SYMMETRIC MATRIX

Unaltered by transposition. $(\tilde{A}=A)$.

SKEW- OR ANTI-SYMMETRIC MATRIX

Equal to negative of its transpose $(\tilde{A}=-A)$. (Elements on principal diagonal are zero.) 
Examp1e:

$$
\left(\begin{array}{rr}
0 & 3 \\
-3 & 0
\end{array}\right)=-\left(\begin{array}{rr}
0 & -3 \\
3 & 0
\end{array}\right)
$$

HERMITIAN MATRIX

Unaltered by taking Hermitian conjugate $\left(A^{*}=A\right)$. ANTI- OR SKEW-HERMITIAN MATRIX

Unaltered by taking negative of Hermitian conjugate. $\left(A^{*}=-A\right)$.

SYMMETRIC AND ANTI-SYMMETRIC COMPONENTS OF A SQUARE MATRIX

A square matrix may be expressed as the sum of two square matrices, one symmetric and the other skew symmetric; or one Hermitian and the other anti-Hermitian.

Example:

$$
\begin{aligned}
A & =\frac{1}{2}(A+\tilde{A})+\frac{1}{2}(A-\tilde{A}) \\
\text { or } A & -\frac{1}{2}(A+A)+\frac{1}{2}\left(A-A^{*}\right) .
\end{aligned}
$$

VARIOUS RULES

$$
\begin{aligned}
& A+B=B+A \\
& A+(B+C)=(A+B)+C \\
& A(B+C)=A B+A C \\
& (A+B) C=A C+B C \\
& A(B C)=\tilde{\sim}(A B) C \\
& \tilde{(A)} \sim \tilde{A}=A \\
& (A B)^{\sim}=\tilde{B A},(A B C)^{\sim}=\tilde{C} \tilde{B A}, \text { etc. }
\end{aligned}
$$




$$
\begin{aligned}
& \overline{(\bar{A})}=A \\
& \overline{A B}=(\bar{A})(\bar{B}) \\
& \tilde{A}=\overline{\widetilde{A}}=A^{*} \\
& \left(A^{-1}\right)^{-1}=A \\
& (A B)^{-1}=B^{-1} A^{-1},(A B C)^{-1}=C^{-1} B^{-1} A^{-1} \text {, etc. } \\
& A^{n} A^{m}=A^{n+m}, n, m=\text { integers; } A^{0} \equiv I .
\end{aligned}
$$

ORTHOGONAL MATRIX

$$
\tilde{\mathrm{A}}=\mathrm{A}^{-1} \text {, or } \tilde{\mathrm{AA}}=\tilde{\mathrm{AA}}=\mathrm{I}
$$

UNITARY MATRIX

$$
A^{-1}=A^{*} \text {, or } A^{*} A=A A^{*}=I \text {. }
$$

(A real unitary matrix is orthogonal.)

DETERMINANT OF A PRODUCT

For square matrices, $\operatorname{det}(A B)=(\operatorname{det} A)(\operatorname{det} B)$.

\section{COLUMN MATRIX}

A matrix with one column, such as

$$
i=\left(\begin{array}{l}
i_{1} \\
i_{2} \\
i_{3}
\end{array}\right)
$$

\section{ROW MATRIX}

A matrix with one row, such as $i^{*}=\left(\begin{array}{lll}\bar{i}_{1} & \bar{i}_{2} & \bar{i}_{3}\end{array}\right)$.

BI LINEAR FORM

$x$ A $y$

$x$ and $y$ are column matrices.

$A$ is a square matrix. 
QUADRATIC FORM

$\tilde{\mathrm{x}} \mathrm{A} x$, where $\tilde{\mathrm{A}}=\mathrm{A}($ symmetric $)$.

HERMITIAN FORM

$x^{*} A x$, where $A^{*}=A$ (Hermitian)

(A Hermitian form is a real number.)

7.6 Appendix F: Linear Dependence, L inear Independence, and the Schwarz Inequality for Complex Vectors

The vectors $A_{1}, \ldots, A_{k}$ are linearly dependent if there exist scalars $c_{1}, \ldots, c_{k}$, not all zero, such that

$$
c_{1-1}+\cdots+c_{k-k} \underline{A}_{k}=0
$$

In the contrary case, i.e., when this equation implies $c_{1}=\cdots=$ $c_{k}=0$, the vectors $\underline{A}_{1}, \cdots, \underline{A}_{k}$ are linearly independent.

The Schwarz inequality for complex vectors states that

$$
|\underline{A} \cdot \underline{B}|^{2} \leq|\underline{A}|^{2}|\underline{B}|^{2}
$$

with equality holding if and only if $\underline{A}$ and $\underline{\bar{B}}$ are linearly dependent (note the occurrence of the superposed bar, indicating the complex conjugate). Of course, the vectors may in particular be real. 


\section{REFERENCES}

[1] Kerns, D. M., Definitions of $v$, i, Z, Y, a, b, $\Gamma$, and S, Proc. IEEE 55, 892-900 (1967).

[2] For a proof of this theorem see Mirsky, L., An Introduction to Linear Algebra, p. 400 ff. (Oxford Universjty Press, 1955).

[3] See, e.g., Kerns, D. M., Basis of the application of network equations to waveguide problems, Nat. Bur. Stand. (U.S.), 42, 515-540 (1949). A more general type of reciprocity theorem (which could also be stated for $N$-ports) will be stated for antennas and scatterers in Chapter II.

[4] Details nay be found in Kerns, D. M., and Beatty, R. W., Basic Theory of Waveguide Junctions and Introductory Microwave Network Analysis, p. 29 ff. (Pergamon, 1967).

[5] Kerns, D. M., Analysis of symmetrical waveguide junctions, J. Res. Nat. Bur. Stand. (U.S.), $267-282$ (1951).

[6] Slater, J. C., Microwave Transmission, p. 66 (McGraw-Hil1, 1942).

[7] Mittra, R., and Richardson, J. L., A numerical technique for solving a class of open-region radiation and scattering problems (Letter), Proc. IEEE 58, $276-279$ (1970).

[8] Yaghjian, A. D., and Kormhauser, E. T., A modal analysis of the dielectric rod antenna excited by the $H_{11}$ mode, IEEE Trans. Antennas Prop. Ap 20, 122-128 (1972).

[9] Mirsky, op. cit.

[10] Kahn, W. K., and Kurss, H., Minimun scattering antennas, IFEE Trans. Antennas and Prop. AP 13, 671-676 (1965). The theory of minimum scattering antennas is discussed briefly in Chapter II of this monograph. 


\section{CHAPTER II}

\section{FORMULATION AND MEASUREMENT APPLICATIONS}

\section{INTRODUCTION}

As already noted in the Preface, this chapter contains all the material in Kerns $[54]^{1}$, with revisions to improve the exposition and with added material making the work more complete and scholarly. In general, references will be made to earlier sources, such as $[1,2]$, rather than to [54].

The plane-wave scattering-matrix formulation is put forth as a good basis for advanced antenna theory, especially such theory related to antenna measurement techniques. While the results given in this chapter may be persuasive, certain theoretical developments and numerous analytical examples that extend and illustrate the theory have been deferred to Chapter III. Apart from the appendices, relatively little theory is developed in this chapter. However, logical interrelations are indicated, and more detail is given when it is a question of relating new concepts to more familiar concepts and to practicable antenna measurement techniques. See, in particular, subsection 1.6 and section 4 of this chapter.

An "acoustics translation" of much of the work of this chapter has been published [3]. Because of the relative simplicity of the acoustic wave-fields involved, the acoustics version may be found helpful in illuminating the electromagnetic case.

Figures in brackets indicate the literature references at the end of this chapter. 
In section 1 we give, quite thoroughly as far as definitions and notation are concerned, a formulation of the plane-wave scattering-matrix (PWSM) for antennas and scatterers. ${ }^{2}$ The complete, "two-sided" formulation, first given explicitly in [34] and [54], is restated in somewhat generalized form.

The scattering matrix formulation properly includes basic expressions for power transfer and for reciprocity.

The reciprocity relations are stated in a generalized form, using the concept of mutually adjoint antennas. The concept of generalized reciprocity is not in itself new $[4,5]$, but apparently it had not previously been formulated for antennas and scatterers (see, however, remark following (1.6-21)). The generalized or adjoint reciprocity relations have found substantial application in research establishing the foundations of the extrapolation technique $[6,7]$. They are used in this chapter in the formulation of possible new antenna measurement techniques, predicated upon the physical realization of mutually adjoint antennas.

In section 2 the plane-wave scattering matrix approach is used to obtain a complete and general solution to the problem of coupled antennas. In spite of their formal appearance, the general results obtained represent the heart of the present theory, and provide a fruitful and reliable basis for additional results (including those reported here). Two of these results are the deconvolution and the extrapolation techniques outlined in section 3. These techniques basically determine values of the scalar product, called the

\footnotetext{
${ }^{2}$ Since an antenna is in general a scattering object, and a passive antenna externally is merely a scattering object; the consideration of scattering is included in the full consideration of antennas and need not always be mentioned explicitly.
} 
coupling product, of two two-component vectors characteristic of the two antennas involved. (This statement also applies to the conventional far-field antenna measurement methods, provided due attention is paid to polarization characteristics.)

Utilization of coupling-product data is discussed as a separate topic. Here the analytical problem is primarily geometric and algebraic, with the exact form depending upon what is considered known a priori and what information is sought. In section 4 formulas for the utilization of coupling-product data, taking full account of polarization characteristics and not requiring reciprocal antennas, are given for (a) one-unknown antenna, (b) generalized two-identical antenna, and (c) generalized threeantenna measurement techniques. The order of 1 isting and discussion is roughly that of decreasing a priori information and increasing complexity.

An increasing body of experimental results involving various combinations of the several techniques identified above may be found in the Iiterature $[8-11,55]$. Moreover, the error analysis required for rational design of facilities and for determination of accuracy in concrete measurement situations is approaching completion (Kanda [12], Yaghjian [13], Newel1 [14]).

The combination of the deconvolution technique with that labelled (a) above amounts to a technique for correction of nearfield antenna measurements made with an arbitrary but known measuring antenna. The ability to obtain the true radiated spectrum of an unknown antenna, fully corrected for the effects of the measuring antenna, incidentally implies the ability to obtain corresponding true values of $\underline{E}$ and $\underline{H}$ at arbitrary distances 
in front of a radiator, similarly fully corrected. Frequently, and in particular in the following paragraphs, the measuring antenna will be referred to as a "probe."

The general subject of detcrmination of far-ficld antenna patterns from near-fjeld data is surveyed and an extensive bibliography is given in a recent paper by Johnson ct a1. [15]. This paper should be consulted for an overvicw of the subject. Here we mention specifically only certain earlier work in which the "probe-correction problem" was considercd or which represented steps leading to the evcntual simple, rigorous, and general deconvolution solution of the problem.

Woonton, in 1953 [16], obtaincd an integral expression for the near-field response of a linear (= thin wire) antenna and discussed probe effects qualitatively. Woonton stated that the problem had not been critically discussed previously. Dayhoff (1956) [17], using scalar waves, plane-wave spectrum analysis, and reciprocity, introduced a version of the very important transmission integral. (Dayhoff used the transmission integral to obtain an approximate solution of a diffraction correction problem in microwave interfcrometry. A rigorous and more gencral version of this solution was prescnted by kerns in 1957 [18].) Brown [19] (1958), using planc-wave spectrum analysis and reciprocity [20], obtained a version of the transmission integral and used it to give an approximatc analysis of probc effects, assuming simple, known data for both antennas involved. Morc work along this 1 inc (1imited to two-dimensjons) was done by Ju]1 [21,22]. In 1.961 Brown and Jull [23] gave a rigorous and general solution to the probe correction problem for the two-dimensional case 
using cylindrical wave functions. The use of two-dimensional solutions in certain three-dimensional problems has been suggested and was studied experimentally by Martin [24], but is not valid for any three-dimensional problems. The proper extension to three dimensions in spherical or cylindrical coordinates is far from trivial. See Jensen [25], Leach and paris [26], and Wacker [27]. The key to the present solution to the probe-correction problem is the use of plane-wave analysis and rectangular coordinates and the recognition that planar scanning would permit solution of the integral equation presented by the transmission integral by Fourier inversion ${ }^{3}$ (better called deconvolution in the existing context). This solution was presented in 1963 by Kerns [28], and again, including application of a two-dimensional, spatial sampling theorem, in 1967, at a University of Colorado Advanced Electromagnetic Theory Summer Course. Archival publication, accompanied by substantial experimental application and verification by Baird et a1. [8], was accomplished in 1970 [2]. The transmission integral used, though similar to that derived by Brown, was actually obtained by methods used in Kerns and Jayhoff [1], where it was derived without recourse to reciprocity and was explicitly recognized as the first term in an infinite series of interaction terms. (See the remarks at the end of Appendix A, below.)

The Kerns and Dayhoff paper, not originally considered to be in the domain of antenna theory by its authors, has served as an important base for much of the material embodied in the present monograph and in other work as yet unpublished.

These matters are adequately discussed later in this chapter. 
1. PLANE-WAVE SCATTERING-MATRIX FOR ANTENNAS NND SCATTERERS

1.1 Representation of Fields on $\mathrm{S}_{\mathrm{O}}$, Definition of $\mathrm{a}_{\mathrm{o}}$ and $\mathrm{b}_{\mathrm{O}}$

Let us consider the antenna system shown schematically in figure 1. We choose a (mathematical) terminal surface $\mathrm{S}_{\mathrm{O}}$ in the waveguide feed and define a supplementary surface $\mathrm{S}_{a}$, such that $\mathrm{S}_{\mathrm{a}}+\mathrm{S}_{\mathrm{o}}$ forms a closed surface enclosing the source or detector associated with the antenna. The surface $S_{a}$ coincides with shielding, which is required to make the problem well defined (both experimentally and theoretically!). As an important measure of simplicity, we consider only the case of a single waveguide feed, supporting just one propagated mode. We employ conventional phasor wave amplitudes $a_{0}$ and $b_{0}$ for the incident and emergent traveling wave components at $S_{0}$. These wave amplitudes are fully defined by the following four equations.

The tangential (= transverse) components of $\underline{\mathrm{E}}$ and $\underline{\mathrm{H}}$ on $\mathrm{S}_{\mathrm{O}}$ are given by

$$
\begin{aligned}
& \underline{E}_{0 t}=\left(a_{0}+b_{0}\right) \underline{e}_{0}(\underline{r}), \\
& \underline{H}_{0 t}=n_{0}\left(a_{0}-b_{0}\right) \underline{h}_{0}(\underline{r}),
\end{aligned}
$$

where $\underline{e}_{0}(\underline{r})$ and $\underline{h}_{0}(\underline{r})$ are real basis fields for the mode involved, subject to the impedance normalization

$$
\underline{h}_{0}(\underline{r})=\eta_{0}^{-1} \eta_{w} n_{0} \times \underline{e}_{0}(\underline{r})
$$

and to the power normalization

$$
\int_{S_{0}}\left[\underline{e}_{0} \underline{h}_{-} \underline{n}_{0}\right] \quad d S=1
$$




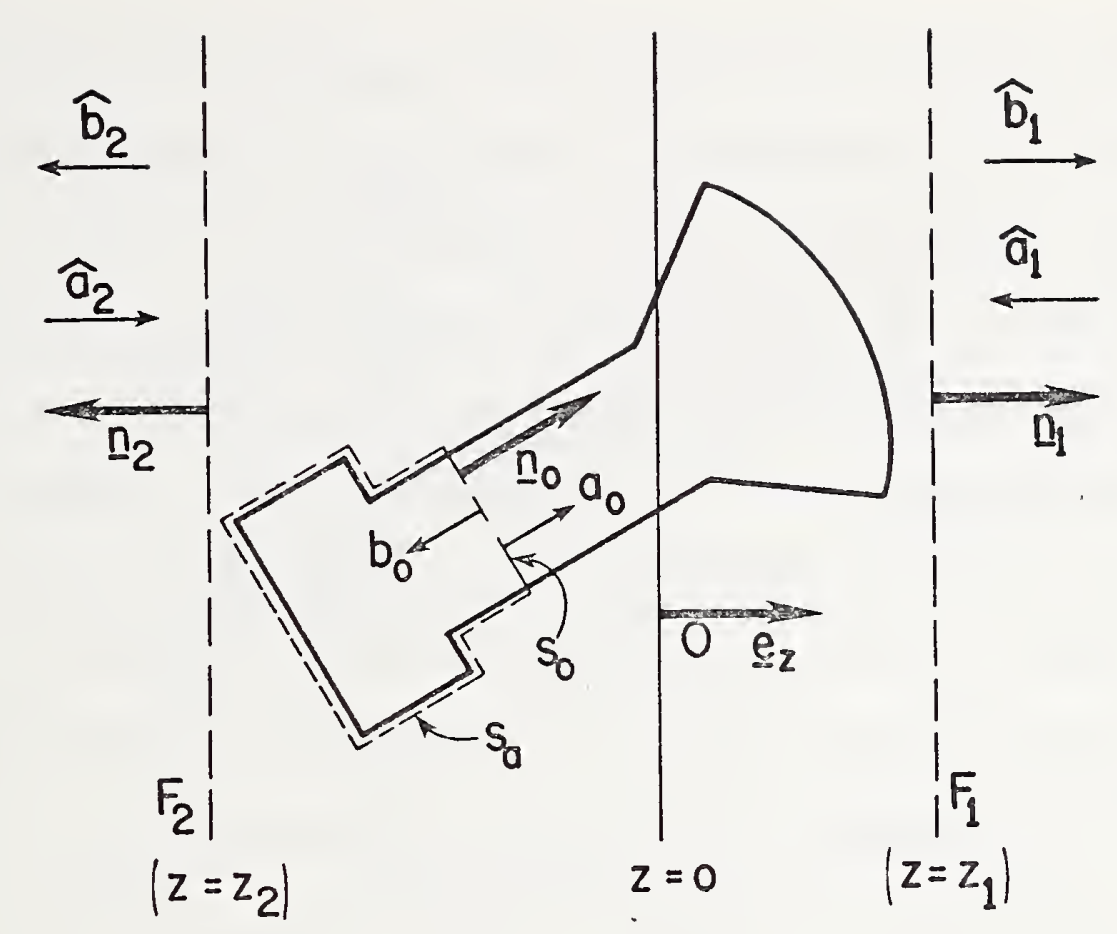

Figure 1. Some notation for plane-wave scattering-matrix description of antennas.

Arrows indicate the association of a's and b's with incident and emergent waves respectively. Antenna-system representation is symbolic: no particular size, shape, symmetry, type, orientation, or position is implied. In any concrete case, position and orientation of antenna relative to cocrdinate system must be established, as with the aid of fiducial marks on the antenna.

Here the integrand is the scalar triple product; $\underline{n}_{0}$ is the unit normal vector, drawn inward with respect to the antenna; $n_{0}$ is the characteristic admittance, and $n_{w}$ is the wave admittance for the mode involved. Equation.(3) establishes peak-value normalization for $a_{0}$ and $b_{0}$, so that net time-average power input to the antenna at $S_{0}$ is given by

$$
P_{0}=\frac{1}{2} \operatorname{Re} \int_{S_{0}}\left[\underline{E}_{0 t} \underline{\bar{H}}_{0 t} \underline{n}_{0}\right] d S=\frac{1}{2} n_{0}\left(\left|a_{0}\right|^{2}-\left|b_{0}\right|^{2}\right),
$$

where Re denotes that the real part is to be taken, the superposed bar denotes the complex conjugate, and the vertical bars denote absolute values. Remarks: (a) The impedance normalization shown above is more flexible than that used previously [1,2]. It allows 
one to choose whatever characteristic impedance or admittance is deemed familiar or convenient for purposes of measurement-related calculations. Examples are, for waveguide, $n_{0}=1$ or $n_{0}=n_{w}$; and, for coaxial line, the conventional characteristic admittance $c=2 \pi \sqrt{\varepsilon / \mu} / \ln (\mathrm{b} / \mathrm{a})$ in conventional notation). (b) Power normalization differs slightly from that used previously: Powers of the factor $2 \pi$ have been redistributed. (c) A detailed discussion of the definitions in this subsection and an introduction to the microwave network theory needed in measurement-related calculations has, it will be recalled, been given in Chapter $\mathrm{I}$.

1.2 Representation of Fields in Space; Definition of $a_{q}(m, K)$ and $\mathrm{b}_{\mathrm{q}}(\mathrm{m}, \underline{\mathrm{K}})$

We choose a rectangular coordinate system oxyz (with unit vectors $\underline{e}_{x}, \underline{e}_{y}$ and $\underline{e}_{z}$ ) so that the considered antenna system may be confined entirely to the space between the (mathematical) surfaces $F_{1}$ and $F_{2}$ at $z=z_{1}$ and at $z=z_{2}$ (where $z_{1}>z_{2}$ ) as suggested in figure 1. The electromagnetic fields in the regions to the "right" and to the "left" are to be represented as superpositions of plane-wave solutions of Maxwell's equations. This type of representation is well known (see e.g., [29], p. $361 \mathrm{ff}$ ), at least for solutions of the scalar Helmholtz equation; an appropriate generalization to the (vector) electromagnetic field, though often shunned, offers no particular difficulty. 


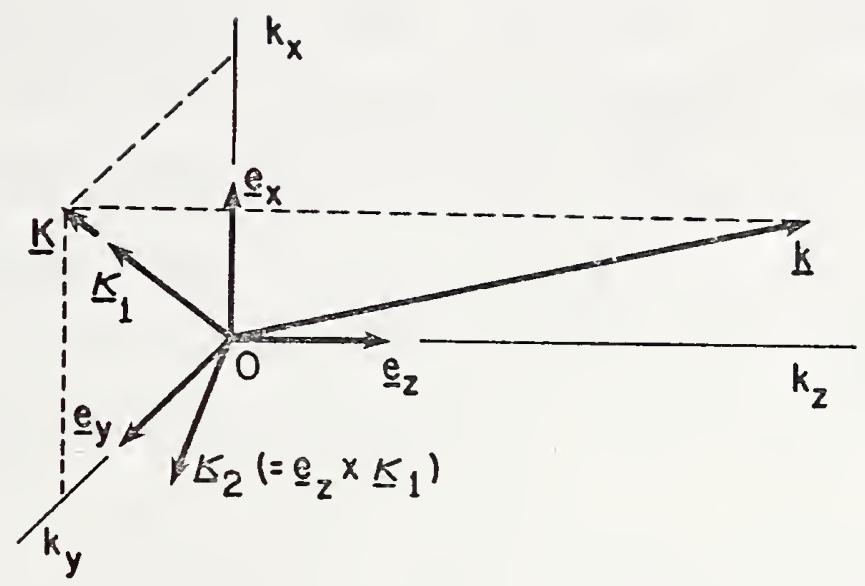

Figure 2. Illustrating $\underline{k}, \underline{K}, \underline{\kappa}_{1}$, and $\underline{\kappa}_{2}$.

The electromagnetic field in the regions under consideration satisfies Maxwell's equations in the form

$$
\nabla \times \underline{E}=i \omega \mu \underline{H}, \quad \nabla \times \underline{H}=-i \omega \varepsilon \underline{E},
$$

where $\mu, \varepsilon$ are constant real scalars representing respectively the

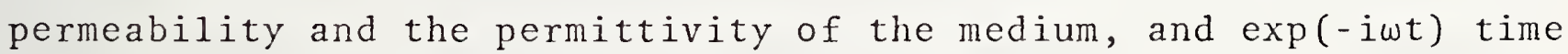
dependence is assumed. We derive our basis fields from the general plane wave

$$
\left.\begin{array}{l}
\underline{E}=\underline{T} \exp (i \underline{k} \cdot \underline{r}), \\
\underline{H}=(\omega \mu)^{-1} \underline{k} \times \underline{T} \exp (\underline{i k} \cdot \underline{r}),
\end{array}\right\}
$$

which is a solution of (1) for any propagation vector $\underline{k}$ such that $\mathrm{k}^{2} \equiv \underline{\mathrm{k}} \cdot \underline{\mathrm{k}}=\omega^{2} \mu \varepsilon$ and any vector $\underline{\mathrm{T}}$ (independent of position $\underline{\mathrm{r}}$ ) satisfying the transversality relation

$$
\underline{k} \cdot \underline{T}=0 .
$$


In spite of this occurrence of "transversality," in what follows the term "transverse" will mean transverse with respect to the z-direction unless otherwise indicated.

The propagation vector will be regarded as a function of its transverse components $k_{x}, k_{y}$ (which are chosen real); the $z-$ component is thus

$$
k_{z}= \pm \gamma
$$

where $r^{2}=k^{2}-k_{x}^{2}-k_{y}^{2}$. It will be convenient to denote the transverse part of the propagation vector by $\underline{K}$, so that $\underline{K}=k_{x} e_{x}+k_{y}-y$ and

$$
\gamma=\left(k^{2}-K^{2}\right)^{\frac{1}{2}}
$$

Since $k_{x}, k_{y}$ must be allowed to vary independently in the range $(-\infty, \infty)$, real and imaginary values of $\gamma$ will occur. $\gamma$ will be taken positive for $K<k$, positive imaginary for $K>k$. Superscripts "+" and "-" will be used on $\underline{k}$ when it is desirea to indicate the choice of $\operatorname{sign}$ associated with $k_{z}$. When $\gamma$ is real, the exponentials $\exp \left(\mathrm{ik}^{+} \cdot \underline{r}\right)$ and $\exp \left(i \underline{\mathrm{k}}^{-} \cdot \underline{\mathrm{r}}\right)$ respectively represent simple plane waves traveling into the $+z$ and $-z$ hemispheres. When $\gamma$ is imaginary, the exponentials represent inhomogeneous plane waves with propagation of phase in the transverse directions and exponential attenuation of amplitude ("evanescence") in the + and - z-directions, respectively.

In virtue of the relation $\underline{k} \cdot \underline{T}=0$, (2a) yields just two lincarly independent fields, hence just two basis fields, for any given $\underline{k}$. The appropriate polarizations for the basis fields are those with the electric vectors parallel or perpendicular to the plane of $\underline{k}$ and $\underline{e}_{z}$, which is the plane of incidence for a wave 
incident on any plane $z=$ const. This choice of polarizations yields "transverse magnetic" and "transverse electric" waves; the same choice of polarizations, usually labelled "E $\mid$ " and "E $\perp$ ", simplifies the derivation of Fresnel's equations in optics or electromagnetic theory.

In order to set up the basis fields in the desired form, we require the transverse unit vectors

$$
\underline{\kappa}_{1}=\underline{K} / K, \quad \underline{\kappa}_{2}=\underline{e}_{2} \times \underline{\kappa}_{1},
$$

which are respectively in and perpendicular to the plane of $\underline{k}$ and $\underline{e}_{z}$. This part of the notation is illustrated in figure $2 ; \underline{\kappa}_{1}$ and $\underline{\kappa}_{2}$ may be identified as radial and tangential unit vectors, as often associated with polar coordinates in a plane. As a temporary abreviation ${ }^{4}$ we put $u^{ \pm}=\exp \left(i \underline{k}^{ \pm} \cdot \underline{r}\right) /(2 \pi)$. For the $E / \mid$ (or TM) components we put $\underline{T}=\underline{\kappa}_{1} \mp \mathrm{Kr}^{-1} \underline{e}_{z}$ and obtain from (2a)

$$
\left.\begin{array}{l}
\underline{E}_{1}^{ \pm}=\left[\underline{\underline{k}}_{1} \mp \mathrm{K \gamma}^{-1} \underline{\mathrm{e}}_{2}\right] \mathrm{u}^{ \pm} \\
\underline{H}_{1}^{ \pm}= \pm n_{1} \underline{\mathrm{e}}_{2} \times \underline{\mathrm{k}}_{1} \mathrm{u}^{ \pm}
\end{array}\right\}
$$

where $n_{1}=\omega \varepsilon / \gamma$. For the $E_{\perp}$ (or TE) components we take $\underline{T}=\underline{\kappa}_{2}$ and obtain from ( $2 a)$

$$
\left.\begin{array}{l}
\underline{\mathrm{E}}_{2}^{ \pm}=\underline{\mathrm{k}}_{2} \mathrm{u}^{ \pm}, \\
\underline{\mathrm{H}}_{2}^{ \pm}=\left[ \pm \eta_{2} \underline{\mathrm{e}}_{\mathrm{z}} \times \underline{\mathrm{k}}_{2}+\mathrm{K}(\omega \mu)^{-1} \underline{\mathrm{e}}_{\mathrm{z}}\right] \mathrm{u}^{ \pm},
\end{array}\right\}
$$

where $n_{2}=\gamma /(\omega \mu)$. Among other similarities it may be observed that $n_{1}, n_{2}$ are wave-admittances that correspond closely to the The factor $1 /(2 \pi)$ in the definition of $u$ represents a change in normalization
consistent with that noted for $a_{0}$ and $b_{0}$ in the preceding subsection. 
wave-admittances encountered in the theory of rectangular waveguide. Equations (5) and (6) furnish the desired basis fields; somewhat arbitrarily, we have chosen to make the expressions for transverse E as simple as possible. The normalization and orthogonality properties of the basis fields are of course implicit in the expressions themselves. (This is an interesting contrast to (1.1-1), where the field patterns are implicit and the normalizations explicit.)

Let us now examine the plane-wave representations of electromagnetic fields in the regions $z \bar{\gamma} z_{1}$ and $z \overline{<} z_{2}$. We write

$\underline{E}_{q}(\underline{r})=\int_{-\infty}^{\infty} \int_{m=1}^{2}\left[b_{c_{i}}(m, \underline{K}) \underline{E}_{m}^{ \pm}(\underline{K}, \underline{r})+a_{q}(m, \underline{K}) \underline{E}_{m}^{+}(\underline{K}, \underline{r})\right] d k_{x} d k_{y},(1.2-7 a)$ $\underline{H}_{q}(\underline{r})=\int_{-\infty}^{\infty} \int_{m=1}^{2}\left[b_{q}(m, \underline{K}) \underline{H}_{m}^{ \pm}(\underline{K}, \underline{r})+a_{q}(m, \underline{K}) \underline{H}_{-m}^{\mp}(\underline{K}, \underline{r})\right] d k_{x} d k_{y} \cdot(1.2-7 b)$ Here the index q takes on the values 1 and 2 and identifies quantities respectively associated with the regions to the "right" and to the "left" of the system considered; the upper and the lower superscript signs are associated with $q=1$ and $q=2$, respectively; and $\mathrm{b}_{\mathrm{q}}(\mathrm{m}, \underline{\mathrm{K}})$ and $\mathrm{a}_{\mathrm{q}}(\mathrm{m}, \underline{\mathrm{K}})$ are scalar spectral-density functions for outgoing and incoming waves, respectively. The electromagnetic fields given by (7) will satisfy Maxwell's equations provided that the necessary differentiations can be taken under the integral signs. Now, as will be shown in a moment, a knowledge of the transverse components of $\underline{E}_{q}$ and $\underline{H}_{q}$ (in a single plane, in fact) is sufficient to determine $\mathrm{a}_{\mathrm{q}}(\mathrm{m}, \mathrm{K})$ and $\mathrm{b}_{\mathrm{q}}(\mathrm{m}, \underline{\mathrm{K}})$; and hence, by (7), the entire electromagnetic field in each of the regions considered. The z-components of the fields in (7) are, strictly speaking, redundant. The transverse components are both necessary and suf- 
ficient for the expression of normal energy-flux and continuity conditions across a transverse plane. In the course of this monograph we shall find, in fact, that the inclusion of $z$-components is sometimes convenient, sometimes inconvenient, and often immaterial.

For the transverse components of $\underline{E}$ and $\underline{H}$ in the regions $z \geq z_{1}$ and $z \leq z_{2}$, we find from (5), (6), and (7) the Fourier transform representations

$$
\begin{array}{r}
\underline{E}_{q t}(\underline{r})=\frac{1}{2 \pi} \int \sum_{m}\left[b_{q}(m, \underline{K}) e^{ \pm i \gamma z}+a_{q}(m, \underline{K}) e^{\mp i \gamma z}\right] \\
\underline{K}_{m} e^{i \underline{K} \cdot \underline{R}} d \underline{K}, \\
\underline{H}_{q t}(\underline{r})=\frac{1}{2 \pi} \int \sum_{m}\left[b_{q}(m, \underline{K}) e^{ \pm i \gamma z}-a_{q}(m, \underline{K}) e^{\mp i \gamma z}\right] \\
\underline{n}_{q} \times \underline{\kappa}_{m} \eta_{m}(K) e^{i \underline{K} \cdot \underline{R}} d \underline{K},
\end{array}
$$

where $\eta_{1}=\omega \varepsilon / \gamma, \eta_{2}=\gamma /(\omega \mu)$, and $\underline{r}=\underline{R}+z \underline{e}_{z}$. Here and in subsequent expressions. of this type summation over the values 1 and 2 of the polarization index $\mathrm{m}$ and integration over the infinite $k_{x}, k_{y}$ plane is to be understood. The role of the index $q$ is as described under (7); $\underline{n}_{\mathrm{q}}$ is the outward normal unit vector on $\mathrm{F}_{\mathrm{q}}$ : $\underline{\mathrm{n}}_{1}=\underline{\mathrm{e}}_{2}, \underline{\mathrm{n}}_{2}=-\underline{\mathrm{e}}_{2}$ (i.e., "outward" is defined with respect to the slab between the surfaces $F_{1}$ and $\left.F_{2}\right)$. The functions $a_{q}(m, K)$ and $b_{q}(m, K)$ may be regarded as modal terminal variables for the continuous spectrum; a mode is identified by a triplet of values $\left(\mathrm{m}, \mathrm{k}_{\mathrm{x}}, \mathrm{k}_{\mathrm{y}}\right.$ ) and a direction (rightward or leftward).

Explicit expressions for the spectral density functions $b_{q}(m, K)$ and $a_{q}(m, \underline{K})$ may be found from the Fourier inversion of the above equations: 
$b_{q}(m, \underline{K})=\frac{e^{\mp i \gamma z}}{4 \pi} \underline{k}_{m} \cdot \int\left[\underline{E}_{q}(\underline{R}, z)+n_{m}^{-1} \underline{H}_{q}(\underline{R}, z) \times \underline{n}_{-q}\right] e^{-i \underline{K} \cdot \underline{R}} d \underline{R}$,

$a_{q}(m, \underline{K})=\frac{e^{ \pm i \gamma z}}{4 \pi} \underline{k}_{m} \cdot \int\left[\underline{E}_{q}(\underline{R}, z)-\eta_{m}^{-1} \underline{H}_{q}(\underline{R}, z) \times \underline{n}_{q}\right] e^{-i \underline{K} \cdot \underline{R}} d \underline{R}$.

$\left(\eta_{1 \pi}^{-1}\right.$, which is independent of the space coordinates, is written inside the integral signs merely to simplify the typography.)

Here the integrations are to be taken over the entire $x, y$ plane-as required by the Fourier inversion--for any suitable fixed value of $z$ (for $q=1, z \geq z_{1}$; for $q=2, z \leq z_{2}$ ). The spectral functions $b_{q}(m, \underline{K})$ and $a_{q}(m, \underline{K})$ are independent of $z$, although this may not be immediately apparent in (9) .

It is convenicnt at this point to introduce a number of definitions for future reference when and as needed.

We observe that (7a) can be written

$$
\underline{E}_{q}(\underline{r})=\frac{1}{2 \pi} \int\left[\underline{b}_{q}(\underline{K}) e^{ \pm i \gamma z}+\underline{a}_{q}(\underline{K}) e^{\mp i \gamma z}\right] e^{i \underline{K} \cdot \underline{R}} d \underline{K} .
$$

Here $\underline{b}_{q}$ and $\underline{a}_{q}$, the "complete vectorial spectra" for the outgoing and incoming plane-wave components of $\underline{E}_{q}$, respectively, are given by

$$
\begin{aligned}
& \underline{b}_{q}(\underline{K})=b_{q}(1, \underline{K})\left(\underline{k}_{1} \mp K \gamma^{-1} \underline{e}_{z}\right)+b_{q}(2, \underline{K}) \underline{\kappa}_{2}, \\
& \underline{a}_{q}(\underline{K})=a_{q}(1, \underline{K})\left(\underline{\kappa}_{1} \pm K \gamma^{-1} \underline{e}_{z}\right)+a_{q}(2, \underline{K}) \underline{\kappa}_{2} .
\end{aligned}
$$

(The association of upper and lower signs with $q=1$ and $q=2$, respectively, originating in (7), will continue, explicitly or implicitly, throughout this monograph.) Alternatively, $\underline{b}_{q}$ and a may be expressed as follows: 
Sec. 1.2]

$$
\begin{aligned}
& \underline{b}_{q}(\underline{K})=b_{q}(1, \underline{K})\left[(-)^{q-1} k / \gamma\right] \underline{e}||(\underline{k})+b_{q}(2, \underline{K}) e \underline{l}(\underline{K}), \\
& \underline{a}_{q}(\underline{K})=a_{q}(1, \underline{K})\left[(-)^{q} k / \gamma\right] \underline{e}||(\underline{k})+a_{q}(2, \underline{K}) \underline{e} \underline{l}(\underline{K}),
\end{aligned}
$$

where $\underline{e} \|(\underline{k})$ is defined as $\underline{k}_{2} \times \underline{k} / k$, and $\underline{e} \underline{l}(\underline{K})$ is a suggestive alternative notation for $\underline{\kappa}_{2}$. (The factors in brackets in (12) are introduced as a convenient, explicit way of helping to keep signs straight.) The complete vectorial spectra are of interest to us primarily in the propagating regime, where $\underline{e}||$, as well as $\underline{e} \perp$, is real.

All the unit vectors associated with $\underline{k}$ and $\underline{K}$ are pictured in figure 3 and fully identified and related in table 1.

The complete vectorial spectra must and do satisfy the transversality relations

$$
\underline{\mathrm{k}}^{ \pm} \cdot \underline{\mathrm{b}}_{\mathrm{q}}=0, \quad \underline{\mathrm{k}}^{\mp} \cdot \underline{\mathrm{a}}_{\mathrm{q}}=0
$$

which indeed are expressions of the transversaiity of the basis fields (5) and (6).

We next observe that (8a) can be written

$$
\underline{E}_{q t}(\underline{r})=\frac{1}{2 \pi} \int\left[\underline{B}_{q}(\underline{K}) e^{\underline{i} i \gamma z}+\underline{A}_{q}(\underline{K}) e^{\mp i \gamma z}\right] e^{i \underline{K} \cdot \underline{R}} d \underline{K},
$$

where $\underline{B}_{q}$ and $\underline{A}_{q}$, the "transverse vectorial spectra" for the outgoing and incoming plane-wave components of $\underline{E}_{q t}$, respectively, are given by

$$
\begin{aligned}
& \underline{B}_{q}(\underline{K})=\sum_{m} b_{q}(m, \underline{K}) \underline{\kappa}_{m}, \\
& \underline{A}_{q}(\underline{K})=\sum_{m} a_{q}(m, \underline{K}) \underline{\kappa}_{m} .
\end{aligned}
$$


Further we note that, given (15), z-components can be recovered with the aid of the transyersality relations (13) or simply by inspection of (11).

The use of the lower case letters $\underline{a}$ and $\underline{b}$ to denote complete spectra and the use of the capitals $\underline{A}$ and $\underline{B}$ to denote the corresponding transverse parts may be noted. This parallels our use of the letters $\underline{\mathrm{r}}, \underline{\mathrm{k}}$ and $\underline{\mathrm{R}}, \underline{\mathrm{K}}$.

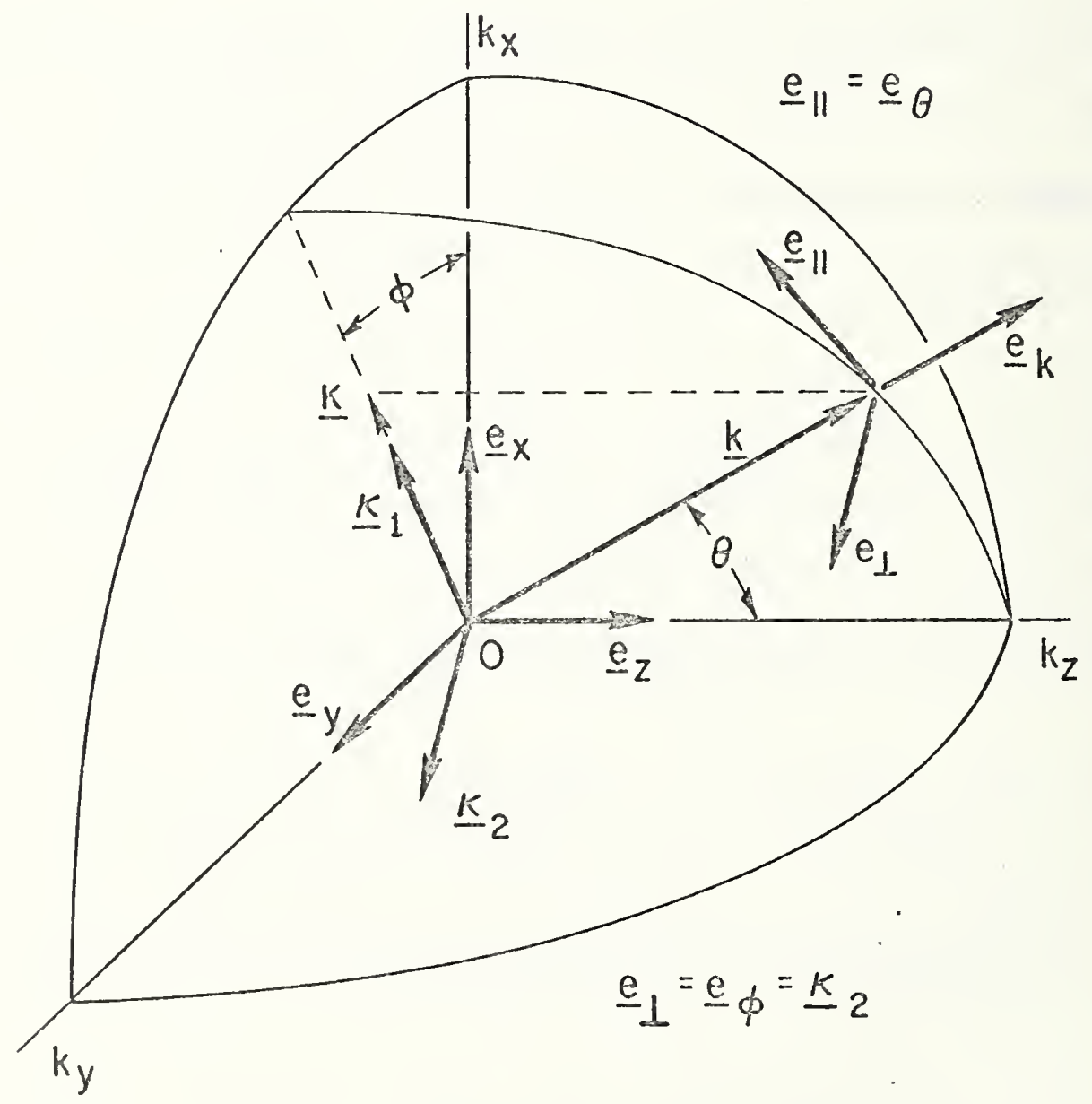

Figure 3. Unit vectors associated with $\underline{k}$ and $\underline{k}$ (for real $\underline{k}$ ). See also Table 1 . 
Table 1. Unit vectors associated with $\underline{K}$ and $\underline{k}$

$$
\underline{K}:\left(k_{x}, k_{y}\right)=(K \cos \phi, K \sin \phi)
$$

$$
\begin{gathered}
\underline{\kappa}_{1} \equiv \underline{K} / K=\left(k_{x} \underline{e}_{x}+k_{y} \underline{e}_{y}\right) / K=\cos \phi \underline{e}_{x}+\sin \phi \underline{e}_{y} \\
\underline{\kappa}_{2} \equiv \underline{e}_{z} \times \underline{\kappa}_{1}=\left(-k_{y} \underline{e}_{x}+k_{x} \underline{e}_{y}\right) / K=-\sin \phi \underline{e}_{x}+\cos \phi \underline{e}_{y} \\
{\left[\underline{\kappa}_{1} \underline{\kappa}_{2} \underline{e}_{z}\right]=1, \quad \underline{\kappa}_{m}(-\underline{K})=-\underline{k}_{m}(\underline{K})}
\end{gathered}
$$

$\underline{k}:\left(k_{x}, k_{y}, k_{z}\right)=(k \sin \theta \cos \phi, k \sin \theta \sin \phi, k \cos \theta)$

$$
\begin{aligned}
& \underline{\mathrm{e}}_{\mathrm{k}} \equiv \underline{\mathrm{k}} / \mathrm{k}=\left(\mathrm{k} \underline{\kappa}_{1}+\mathrm{k}_{\mathrm{z}} \underline{\mathrm{e}}_{z}\right) / \mathrm{k}=\sin \theta \underline{\kappa}_{1}+\cos \theta \underline{\mathrm{e}}_{z} \\
& \underline{e}_{1} \equiv \underline{\kappa}_{2} \times \underline{e}_{k}=\left(k_{z} \underline{\kappa}_{1}-K \underline{e}_{z}\right) / k=\cos \theta \underline{\kappa}_{1}-\sin \theta \underline{e}_{z} \\
& \underline{e} \perp \equiv \underline{\kappa}_{2} \\
& {\left[\underline{e}_{\|} \mid \underline{e}_{1} \underline{e}_{k}\right]=1, \quad \underline{e}\|(-\underline{k})=\underline{e}\|(\underline{k}), \quad k_{z}= \pm \gamma}
\end{aligned}
$$

Notes:

(a) $\underline{K}_{1}$ and $\underline{K}_{2}$ are respectively "radial" and "tangential" unit vectors associated with the plane polar coordinates for $\underline{K}$. These unit vectors are always rea1.

(b) In the propagating regime, where $\underline{e} \|$ as well as $\underline{e} 1$ is real, $\underline{e} \|$

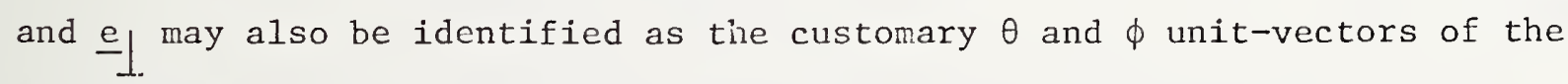
spherical polar coordinates for $k$. The "middle" forms in the table help show what happens when $\mathrm{k}>\mathrm{k}: \underline{e}_{\|}$and $\underline{e}_{\mathrm{k}}$ become complex, but remain unit vectors in the sense $\underline{e}\|\cdot \underline{e}\|=\underline{e}_{k} \cdot \underline{e}_{k}=1$.

(c) The unit vectors $\underline{\kappa}_{1}, \underline{\kappa}_{2}$, and $\underline{e} \mid$ | are not defined by the equations in the table at the singular points $K=0$ and $\theta=0, \pi$. For $\underline{k}=k \underline{e}_{z}$, one may e.g., determine a consistent set by choosing $\underline{k}_{1}=\underline{e}_{x}$. (cf. . use of $(4 \cdot 1-4)$ ). 
The representations (7) and (8) afford a similar and essential resolution of any electromagnetic field into two major parts: that represented by incoming waves and that represented by outgoing waves. The role of the former part is identified variously by terms such as incident, exciting, primary, or incoming; the latter, by terms such as induced, secondary, radiated, reradiated, scattered, or outgoing. The scattering matrix, defined in the next section, will be seen to be a way of specifying the linear transformation from the first part of the field to the second.

The asymptotic relation between the far-field values of a scattered or radiated field and its spectrum is of essential interest and importance. This relation reveals the result of the interference among the waves of the continuous spectrum at large distances in any chosen direction of observation. Normally this interference results (remarkably) in the we11-known $\exp (\mathrm{ikr}) / \mathrm{r}$ variation with distance. This normal result does not apply to the spectrum of a plane wave, and it might not apply to the radiation or reradiation from a source of infinite size in one or more dimensions. For an antenna of finite size radiating into 3-dimensional space, we do have the asymptotic relations (see Appendix $B$, Chapter III)

$$
\begin{aligned}
& \underline{E}_{q t}^{r}(\underline{r}) \sim-i k|\cos \theta| \underline{B}_{q}(\underline{R k} / r) e^{i k r} / r, \\
& \underline{E}_{q}^{r}(\underline{r}) \sim-i k|\cos \theta| \underline{b}_{q}(\underline{R k / r}) e^{i k r} / r .
\end{aligned}
$$

Here the superscript "r" refers to the radiated or reradiated component of the field and $\theta$ is the polar angle of $\underline{r}$ with respect to 
the $z$-axis. The first of the two equations relates to (14) and the second to (10); they differ only in the presence or absence of the $z$-component. Both equations are valid whether or not incident waves are also present in the field. Note that we have written $\underline{R} k / r$ for $\underline{K}$ as the argument of the spectral functions. This expresses the fact that the vectors $\underline{r}$ and $\underline{k}$ involved must be parallel. In fact if we introduce spherical coordinates for $\underline{r}$ such that $x=r \sin \theta \cos \phi, y=r \sin \theta \sin \phi, z=r \cos \theta$, we see that $\underline{b}_{-q}$ and $\underline{B}_{q}$ are expressed as functions of the angular coordinates of $\underline{r}$. Furthermore we note that here only real directions of propagation come into consideration, so that $\gamma$ is real and $\gamma=k|\cos \theta|$ is valid.

To conclude this subsection, we give a useful, non-physical, special result, which offers a sharp contrast to the type of results given just above. Namely, if an electromagnetic field is everywhere the (simple or evanescent) plane wave with $\underline{E}=$ $\underline{a} \exp \left(i \underline{k}_{0}^{-} \cdot \underline{r}\right) / 2 \pi$, then from (9) the corresponding spectral functions are found to be

$$
\left.\begin{array}{l}
a_{1}(m, \underline{K})=b_{2}(m, \underline{K})=a_{m} \delta\left(\underline{K}-\underline{K}_{O}\right), \\
b_{1}(m, \underline{K})=a_{2}(m, \underline{K})=0,
\end{array}\right\}
$$

where $\underline{a}_{m} \equiv \underline{k}_{m} \cdot \underline{a}$ and $\delta\left(\underline{K}-\underline{K}_{0}\right)$ is an abbreviation for the deltafunction product $\delta\left(k_{x}-k_{o x}\right) \delta\left(k_{y}-k_{o y}\right)$. The vector

$$
\underline{A} \equiv a_{1} \underline{K}_{1}+a_{2} \underline{K}_{2}
$$


will be known as the transverse spectral-amplitude vector associated with the considered plane wave. We must, of course, distinguish between spectral amplitudes such as $a_{m}$ and spectral densities such as $a_{1}(\mathrm{~m}, \underline{K})$.

\subsection{Definition of Scattering Matrices for Antennas and Scatterers}

Let us first consider that a passive material structure, exhibiting linear electromagnetic behavior, is present in the region $z_{1}<z<z_{2}$. The scattering equations are written

$$
b_{q}(m, \underline{K})=\sum_{p} \int_{\underline{L}} \sum_{n} S_{q p}(m, \underline{K} ; n, \underline{L}) a_{p}(n, \underline{L}) d \underline{L}, \quad(q=1,2) \quad(1.3-1)
$$

where, in addition to the summation and integration conventions noted following (1.2-8), we have summation over the index $p$, giving the contributions from the waves incident both from the "right" and from the "left." The processes described by the functions $\mathrm{S}_{11}$ and $\mathrm{S}_{22}$ will be called cis-scattering; those described by $\mathrm{S}_{12}$ and $\mathrm{S}_{21}$ will be called trans-scattering. (Regarding the choice of terms: compare, e.g., with cis- and trans-montane, respectively.) Essentially the same definitions and an example of (1) may be found in [1].

If the scattering object is also an antenna, the scattering matrix must include the transmitting and receiving characteristics, and the scattering equations are written

$$
\begin{aligned}
& \mathrm{b}_{\mathrm{o}}=\mathrm{S}_{\mathrm{Oo}} \mathrm{a}_{\mathrm{o}}+\sum_{\mathrm{p}} \int_{\underline{L}} \sum_{n} \mathrm{~S}_{\mathrm{op}}(\mathrm{n}, \underline{\mathrm{L}}) \mathrm{a}_{\mathrm{p}}(\mathrm{n}, \underline{\mathrm{L}}) \mathrm{d} \underline{\mathrm{L}}, \quad(1 \cdot 3-2 \mathrm{a}) \\
& \mathrm{b}_{\mathrm{q}}(\mathrm{m}, \underline{K})=\mathrm{S}_{\mathrm{qO}}(\mathrm{m}, \underline{K}) \mathrm{a}_{\mathrm{o}}+\sum_{\mathrm{p}} \int_{\underline{L}} \sum_{\mathrm{n}} \mathrm{S}_{\mathrm{qp}}(\mathrm{m}, \underline{K} ; \mathrm{n}, \underline{\mathrm{L}}) \mathrm{a}_{\mathrm{p}}(\mathrm{n}, \underline{\mathrm{L}}) \mathrm{d} \underline{\underline{L}} \cdot(1.3-2 \mathrm{~b})
\end{aligned}
$$


Here $\mathrm{q}=1,2$ and we have made use of the quantities $\mathrm{b}_{0}$ and $\mathrm{a}_{0}$, defined in (1.1-1). The quantity $\mathrm{S}_{\text {oo }}$ represents "backscattering" observed at $\mathrm{S}_{\mathrm{O}}$ in the feed waveguide, and the functions $\mathrm{S}_{\mathrm{oq}}(\mathrm{m}, \underline{K})$ and $S_{\text {qo }}(\mathrm{m}, K)$ respectively represent the receiving and the transmitting characteristics of the antenna. (The quantities bearing the subscripts $q$ or $p=2$ in (2) represent the desired generalization of the antenna scattering matrix originally defined by Kerns and Dayhoff [1].)

The definition of the antenna scattering matrix is now literally complete. We do not wish to belabor the generality of the definition, but we do call attention to the absence of restrictive assumptions. At best, its full significance can be made apparent only gradually.

It will be advantageous to have the basic scattering equations expressed in vector-dyadic form. In addition to the transverse vectors $\underline{B}_{q}$ and $\underline{A}_{q}$ defined in $(1.2-15)$, we introduce the vectorial transmitting characteristic

$$
\underline{\mathrm{S}}_{\mathrm{qo}}(\underline{K})=\sum_{\mathrm{m}} \mathrm{S}_{\mathrm{qO}}(\mathrm{m}, \underline{\mathrm{K}}) \underline{\mathrm{K}}_{\mathrm{m}},
$$

the vectorial receiving characteristic

$$
\underline{S}_{o q}(\underline{K})=\sum_{m} S_{o q}(m, \underline{K}) \underline{K}_{m},
$$

and the dyadic scattering characteristic

$$
\underline{\underline{S}}_{q p}(\underline{K}, \underline{L})=\sum_{m, n} S_{q p}(m, \underline{K} ; n, \underline{L}) \underline{\kappa}_{m} \underline{\lambda}_{n},
$$

where the $\underline{\kappa}_{m}$ and $\underline{\lambda}_{n}$ are the unit vectors associated respectively with $\underline{K}$ and $\underline{L}$. The scattering equations become 


$$
\begin{gathered}
b_{0}=S_{o o} a_{0}+\sum_{p} \int \underline{S}_{o p}(\underline{K}) \cdot \underline{A}_{p}(\underline{K}) d \underline{K}, \\
\underline{B}_{q}(\underline{K})=\underline{S}_{q 0}(\underline{K}) a_{0}+\sum_{p} \int \underline{S}_{q p}(\underline{K}, \underline{L}) \cdot \underline{A}_{p}(\underline{L}) d \underline{L} .
\end{gathered}
$$

The equations are now invariant with respect to choice of coordinates in the transverse plane. This facilitates discussion of "real world" cases, in which it is neither expedient nor necessary always to observe or produce pure $\underline{\kappa}_{1}$ or $\underline{\kappa}_{2}$ field components.

Example 1.3-1: Do a dimensional analysis of the quantities in the antenna scattering-equations to find $\left[a_{0}\right],\left[S_{q o}\right],\left[a_{q}(m, \underline{K})\right]$, etc. Preferably make use of the dimensions of appropriate derived units such as volts, rather than using exclusively dimensions of primary quantities such as mass, length, and time. Will the units of $a_{0}$ be volts? Does it matter? (Here, as in subsection 4.3 of Chapter I, we use the symbol [A] to mean "the dimensions of A.")

One may obtain an analysis of the scattering equations by considering the simplest modes of excitation: by a wave represented by a alone, and by individual spatial plane waves, represented by delta-function spectra. This procedure leads essentially to re-expressions of the basic definitions contained in the scattering equations. The circumstances of the resulting definitions or expressions are simple enough to suggest several more or less direct and conventional methods of measurement for the scattering-matrix elements. Let us in particular consider the definitions of $\underline{S}_{q o}(\underline{K})$ and $\underline{S}_{\text {oq }}(\underline{K})$ - - the scattering-matrix quantities that will receive the most attention in our work. Let us consider an antenna operating in its transmitting mode; that is, an antenna excited only by an incident wave in its 
waveguide feed and radiating into empty space. This elementary pattern of excitation is represented by $a_{0} \neq 0, a_{q}(m, K) \equiv 0$. Under these conditions, the spectra radiated (to the left and to the right), normalized to unit $a_{0}$, characterize the transmitting properties of the antenna; indeed, from (4b)

$$
\underline{S}_{q 0}(\underline{K})=\frac{\underline{B}_{q}(\underline{K})}{a_{0}} \text {. }
$$

Incident spatial waves being absent, (1.2-9a) and (1.2-9b) together imply that $\underline{B}_{q}$ may be related either to $\underline{E}$ or to $\underline{H}$; in terms of $\underline{E}$ we have

$$
\underline{S}_{q 0}(\underline{K})=\frac{e^{\mp i \gamma z}}{2 \pi a_{0}} \int \underline{E}_{q t}(\underline{R}, z) e^{-i \underline{K} \cdot \underline{R}} d \underline{R},
$$

where $z>z_{1}$ or $z<z_{2}$ must be in force. This gives us a Fourier transform definition of $\underline{S}_{q 0}$, and, to the extent that $\underline{E}_{q t} / a_{0}$ is known or measurable, a means of calculating $\underline{S}_{q_{0}}$. The asymptotic relation (1.2-16a), applied in the present circumstances, may be written

$$
\underline{E}_{q t}(\underline{r}) \sim-i r \underline{S}_{q 0}(\underline{R k / r}) a_{0} e^{i k r} / r
$$

Clearly this relation may be regarded as a formula for determining $\underline{S}_{q 0}(K)$ in terms of observed asymptotic $\underline{E}_{-q t} / a_{0}$. It in fact represents the basis of so-called direct methods of measurement of transmitting characteristics.

The combination of (6) and (7) incidentally furnishes a rigorous, vectorial form of the Fourier-transform relation between far- and near-fields. Approximate forms of this relation are frequently found in the literature.

Next, consider an antenna excited solely by a spatial plane wave, incident on side $q$, say, and having $\underline{E}=\underline{\alpha} \exp (i \underline{k} \cdot \underline{r}) / 2 \pi$. 
The pattern of excitation is accordingly represented by $a_{0}=0$ and $a_{q^{\prime}}\left(m, \underline{K}^{\prime}\right)=a_{m} \delta q^{\prime} q^{\delta}\left(\underline{K}^{\prime}-\underline{K}\right)$, where $a_{m} \equiv \underline{K}_{m} \cdot \underline{a}$ and the spectrum of the incident wave is found just as in (1.2-17). The scattering equations (2a) or (4a) now yield

$$
\begin{aligned}
b_{0} & =S_{o q}(1, \underline{K}) a_{1}+S_{o q}(2, \underline{K}) a_{2}, \\
& =\underline{S}_{O q}(\underline{K}) \cdot \underline{A} .
\end{aligned}
$$

In other words, $\mathrm{S}_{\mathrm{oq}}(\mathrm{m}, \underline{\mathrm{K}})$ denotes the receiving sensitivity--or receptivity-of the antenna to the $\underline{\kappa}_{m}$-component of polarization of a plane wave incident on the antenna with direction of incidence specified by $\mathrm{q}$ and $\underline{K}$. The normalization is to unit $a_{\mathrm{m}}$ (which means unit delta-function spectral density).

Equation (8) immediately suggests basic equations for direct measurement of receiving characteristics. Viz.,

$$
\begin{aligned}
& b_{O}^{\prime}=\underline{S}_{O q}(\underline{K}) \cdot \underline{A}^{\prime}, \\
& b_{O}^{\prime \prime}=\underline{S}_{O q}(\underline{K}) \cdot \underline{A}^{\prime \prime},
\end{aligned}
$$

where $b_{0}^{\prime}, b_{0}^{\prime \prime}$ are observed anć $\underline{A}^{\prime}, \underline{A}^{\prime \prime}$ are known and linearly independent. The equations can, of course, be solved for $\underline{S}_{o q}$; an algebraically identical problem is encountered in subsection 4.1.

The following compact notation for the scattering equations, to be secured by introducing function vectors and making more use of matrix-algebraic concepts, provides a perspective quite different from that of the discussion just preceding. We first define the column matrices 


$$
\left(\begin{array}{l}
a_{0} \\
\hat{a}_{1} \\
\hat{a}_{2}
\end{array}\right),\left(\begin{array}{l}
b_{0} \\
\hat{b}_{1} \\
\hat{b}_{2}
\end{array}\right)
$$

in which $\hat{a}_{q}$ and $\hat{b}_{q}$ may themselves be regarded as column matrices representing the functions $a_{q}(m, \underline{K})$ and $b_{q}(m, K)$. That is to say, the elements of these column matrices are labelled or indexed according to the values of $\mathrm{m}$ and $\underline{K}$, and have the values $a_{q}(\mathrm{~m}, \underline{K})$ and $b_{q}(m, \underline{K})$, respectively. The transformation from the entire set of incident waves to the entire set of emergent waves is now written

$$
\left(\begin{array}{l}
b_{0} \\
\hat{b}_{1} \\
\hat{b}_{2}
\end{array}\right)=\left(\begin{array}{lll}
s_{00} & \hat{s}_{01} & \hat{s}_{02} \\
\hat{s}_{10} & \hat{s}_{11} & \hat{s}_{12} \\
\hat{s}_{20} & \hat{s}_{21} & \hat{s}_{22}
\end{array}\right)\left(\begin{array}{l}
a_{0} \\
\hat{a}_{1} \\
\hat{a}_{2}
\end{array}\right)
$$

or, equivalently, after performing the indicated matrix multiplication,

$$
\begin{aligned}
& b_{0}=\hat{s}_{00} a_{0}+\hat{s}_{01} \hat{a}_{1}+\hat{s}_{o 2} \hat{a}_{2}, \\
& \hat{b}_{1} \hat{s}_{10} a_{0}+\hat{s}_{11} \hat{a}_{1}+\hat{s}_{12} \hat{a}_{2}, \\
& \hat{b}_{2}=\hat{s}_{20} a_{0}+\hat{s}_{21} \hat{a}_{1}+\hat{s}_{22} \hat{a}_{2} .
\end{aligned}
$$

Here the four kinds of products involved are defined by comparison with (2) or (4). This compact notation, in which the subscripts identify the three input-output reference surfaces, makes the overall structure of the equations more apparent and is a practical necessity for general and formal calculations such as those in the 
next section and in section III-4. The rules of matrix algebra apply: the $\hat{\mathrm{S}}_{\mathrm{qo}}$ (as well as $\hat{\mathrm{a}}_{q}$ and $\hat{\mathrm{b}}_{\mathrm{q}}$ ) correspond to column matrices; the $\hat{\mathrm{S}}_{\mathrm{oq}}$, to row matrices; and the $\hat{\mathrm{S}}_{\mathrm{pq}}$, to square matrices.

We should notice the form that the scattering equations take in the absence of any scattering object (or conceivably in the presence of a non-scattering object). The free passage of waves is expressed by

$$
\mathrm{b}_{1}(\mathrm{~m}, \underline{\mathrm{K}}) \equiv \mathrm{a}_{2}(\mathrm{~m}, \underline{\mathrm{K}}), \quad \mathrm{b}_{2}(\mathrm{~m}, \underline{\mathrm{K}}) \equiv \mathrm{a}_{1}(\mathrm{~m}, \underline{\mathrm{K}}) .
$$

(No propagation factors of the form $\exp ( \pm i \gamma d)$ appear here because the phase reference surfaces were chosen coincident at $z=0$ ). The pertinent sub-matrix in (11) is

$$
\left(\begin{array}{ll}
\hat{S}_{11} & \hat{\mathrm{S}}_{12} \\
\hat{\mathrm{S}}_{21} & \hat{\mathrm{S}}_{22}
\end{array}\right)=\left(\begin{array}{ll}
\hat{0} & \hat{1} \\
\hat{1} & \hat{0}
\end{array}\right) .
$$

Here $\hat{1}$ denotes the identity transformation with elements $\delta_{m n} \delta\left(k_{x}-l_{x}\right) \delta\left(k_{y}-l_{y}\right)$ and $\hat{0}$ denotes the zero transformation. One may regard a scattering object as producing a perturbation of the properties of free-space described in (13) or (14). From this viewpoint, what we may call true or bona fide trans-scattering is described by the operators $\hat{S}_{12}-\hat{1}$ and $\hat{S}_{21}-\hat{I}$. One may compare (I-6.4-11), (I-6.5-4), and the above equation. In the order mentioned, these equations exhibit scattering matrices for free transmission of 1 mode, $\mathrm{N}$ modes, and continua of modes, respectively. 
Example 1.3-2: A 2-port described by the scattering equations

$$
\begin{aligned}
& \mathrm{b}_{0}^{\prime}=\mathrm{T}_{00} \mathrm{a}_{0}^{\prime}+\mathrm{T}_{01} \mathrm{a}_{1}, \\
& \mathrm{~b}_{1}=\mathrm{T}_{10} \mathrm{a}_{0}^{\prime}+\mathrm{T}_{11} \mathrm{a}_{1},
\end{aligned}
$$

is connected to an antenna described by the scattering equations (12) and the combination is considered as a new antenna. The joining equations are $a_{1}=b_{0}, b_{1}=a_{0}$. For simplicity take $\hat{a}_{2} \equiv 0$. Then scattering equations for $b_{0}^{\prime}$ and $\hat{b}_{1}$ are

$$
\begin{aligned}
& b_{0}^{\prime}=\left(\mathrm{T}_{00}+\frac{\mathrm{T}_{01} \mathrm{~T}_{10} \mathrm{~S}_{00}}{1-\mathrm{T}_{11} \mathrm{~S}_{00}}\right) \mathrm{a}_{0}^{\prime}+\frac{\mathrm{T}_{01} \hat{\mathrm{S}}_{01}}{1-\mathrm{T}_{11} \mathrm{~S}_{00}} \hat{\mathrm{a}}_{1} \\
& \hat{b}_{1}=\frac{\mathrm{T}_{10} \hat{\mathrm{S}}_{10}}{1-\mathrm{T}_{11} \mathrm{~S}_{00}} \mathrm{a}_{0}^{\prime}+\left(\hat{\mathrm{S}}_{11}+\frac{\mathrm{T}_{11} \hat{\mathrm{S}}_{10} \hat{\mathrm{S}}_{01}}{1-\mathrm{T}_{11} \mathrm{~S}_{00}}\right) \hat{\mathrm{a}}_{1}
\end{aligned}
$$

Note that in the product $\hat{\mathrm{S}}_{10} \hat{\mathrm{S}}_{01}$ the order of the factors is material.

\subsection{Power Expressions}

The requisite expression for one-mode power transfer in the antenna feed waveguide was given in (1.1-4). Here we shali give the corresponding expressions for power transfer across the surfaces $\mathrm{F}_{1}$ and $\mathrm{F}_{2}$.

The net time-average energy flux across the surface $F_{q}$ in the outward direction (the direction of $\underline{n}_{q}$ ) is given by

$$
\mathrm{P}_{\mathrm{q}}=\frac{1}{2} \operatorname{Re} \int_{\mathrm{F}}[\underline{E \bar{H} \mathrm{n}} \mathrm{q}] \mathrm{d} \underline{\mathrm{R}},
$$

where the integrand is the scalar triple product and the superposed bar denotes the complex conjugate. We wish to evaluate $\mathrm{P}_{\mathrm{q}}$ 
in terms of the spectral density functions $a_{q}(m, \underline{K})$ and $b_{q}(m, \underline{K})$. The calculation is facilitated by the use of the temporary abbreviations

$$
\begin{aligned}
& \underline{V}_{q}(\underline{K})=\sum_{m}\left[b_{q}(m, \underline{K}) e^{ \pm i \gamma z}+a_{q}(m, \underline{K}) e^{\mp i \gamma z}\right] \underline{\kappa}_{m}, \\
& \underline{I}_{q}(\underline{K})=\sum_{m}\left[b_{q}(m, \underline{K}) e^{ \pm i \gamma z}-a_{q}(m, \underline{K}) e^{\mp i \gamma z}\right] \underline{n}_{q} \times \underline{\kappa}_{m} \eta_{m}(K),
\end{aligned}
$$

which enable us to write (1.2-8) in the form

$$
\begin{aligned}
& \underline{E}_{\mathrm{q} t}(\underline{r})=\frac{1}{2 \pi} \int \underline{V}_{\mathrm{q}}(\underline{K}) \mathrm{e}^{i \underline{K} \cdot \underline{R}} \mathrm{dK}, \\
& \underline{H}_{\mathrm{q} t}(\underline{r})=\frac{1}{2 \pi} \int \underline{I}_{\mathrm{q}}(\underline{K}) \mathrm{e}^{i \underline{K} \cdot \underline{R}} \mathrm{~d} \underline{K} .
\end{aligned}
$$

These equations represent $\underline{E}_{q t}$ and $\underline{H}_{q t}$ in terms of vectorial "voltage" and "current" spectral densities and the scalar waves $\exp (\underline{i} \cdot \underline{R}) /(2 \pi)$. The power orthogonality and normalization of these waves are exhibited in the expression

$$
\frac{1}{(2 \pi)^{2}} \int e^{i\left(\underline{K}-\underline{K}^{\prime}\right) \cdot \underline{R}} d \underline{R}=\delta\left(\underline{K}-\underline{K}^{\prime}\right) \text {. }
$$

(This is also a key relation in the derivation of the reciprocity lemma (A-7) in Appendix A.) Hence

$$
P_{q}=\frac{1}{2} \operatorname{Re} \int\left[\underline{V}_{q}(\underline{K}) \bar{I} \underline{I}_{q}(\underline{K}) \underline{n}_{q}\right] d \underline{K} .
$$

Next, using the vector relation $\underline{\kappa}_{m} \times\left(\underline{n}_{q} \times{ }_{-n}\right) \cdot \underline{n}_{q}=\delta_{m n}$ and the notation $\gamma=i \alpha+\beta$ (as in subsection 2.2 of Chapter I) we obtain

$$
\begin{aligned}
P_{q}=\frac{1}{2} \int \sum_{m} & {\left[\left(\left|b_{q}\right|^{2} e^{\mp 2 \alpha z}-\left|a_{q}\right|^{2} e^{ \pm 2 \alpha z}\right) \operatorname{Re}\left(\eta_{m}\right)\right.} \\
& \left.+2 \operatorname{Im}\left(5_{q} a_{q} e^{\mp 2 i \beta_{z}}\right) \operatorname{Im}\left(\eta_{m}\right)\right] d \underline{K} .
\end{aligned}
$$


Here $\mathrm{a}_{\mathrm{q}}$ and $\mathrm{b}_{\mathrm{q}}$ are understood to be functions of $\mathrm{m}$ and $\underline{\mathrm{K}}$. As written, (7) is valid for a lossy (or "gainy") medium for which both $\gamma$ and $n_{m}$ would have both real and imagirary parts for all $\underline{K}$. Perhaps the worst feature of the expression is that then $\mathrm{P}_{\mathrm{q}}$ would depend upon $z$. However, we do not wish to enter into the consideration of lossy media at this point. For a lossless medium, $\gamma$ and $n_{m}$ are both real in the propagating region $(K<k)$ and both imaginary in the evanescent region $(K>k)$, and (7) may accordingly be written

$$
\begin{aligned}
P_{q}=\frac{1}{2} \underset{K<k}{ } \sum_{m}\left[\left|b_{q}(m, \underline{K})\right|^{2}-\right. & \left.\left|a_{q}(m, \underline{K})\right|^{2}\right] n_{m}(K) d \underline{K}+ \\
& -\int_{K>k} \sum_{m} \operatorname{Re} \overline{\left[b_{q}(m, \underline{K}) a_{q}(m, \underline{K}) n_{m}(K)\right] d \underline{K}} .
\end{aligned}
$$

Although the $z$-dependence is no longer present, we see that even in the lossless medium power may be transferred by coupling of incoming and outgoing evanescent modes having the same $m$ and $\underline{k}$. (This is the essential mechanism of power transfer in, e.g., a waveguide-below-cutoff attenuator.) We expect and assume that ordinarily this interaction will be neglibible, as in the case of coupling of ordinary waveguide junctions; the separation required is measured in wavelengths, not Rayleigh distances. Thus the basic expression ( 8 ) may ordinarily be abbreviated to

$$
\mathrm{P}_{\mathrm{q}}=\frac{1}{2} \int_{\mathrm{K}<\mathrm{k}} \sum_{\mathrm{m}}\left[\left|\mathrm{b}_{\mathrm{q}}(\mathrm{m}, \underline{\mathrm{K}})\right|^{2}-\left|\mathrm{a}_{\mathrm{q}}(\mathrm{m}, \underline{\mathrm{K}})\right|^{2}\right] \eta_{\mathrm{m}}(\mathrm{K}) \mathrm{d} \underline{\mathrm{K}} .
$$

The terms in (8) and (9) may often be conveniently abbreviated by using a notation for Hermitian and anti-Hermitian scalar products 


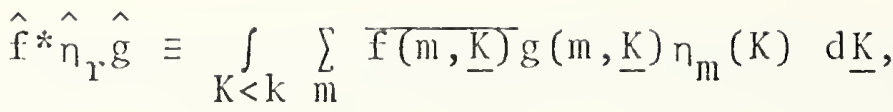

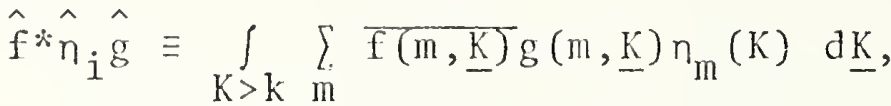

where $f$ and $g$ may be any two scalar functions of $m$ and $k$. We have chosen this matrix style of notation in accordance with the concept of $\hat{\mathrm{a}}_{\mathrm{q}}$ and $\hat{\mathrm{b}}_{\mathrm{q}}$ as column function-vectors introduced in subsection 1.3. In the expressions displayed immediately above, the distinct ranges of integration involved are indicated by the use of the symbols $\hat{n}_{r}$ and $\hat{n}_{i}$, which represent diagonal transformations (or weight functions). $\hat{n}_{\mathrm{r}}$ symbolizes the real (and positive) values of $\eta_{m}$ occurring in (10a), whereas $\hat{\eta}_{i}$ symbolizes the pure imaginary values of $\eta_{m}$ that occur in $(10 \mathrm{~b})$.

Example 1.4-1: Show that the $\mathrm{P}_{\mathrm{q}}$ of (9) are also given by

$$
\mathrm{P}_{\mathrm{q}}=\frac{\mathrm{Y}_{\mathrm{O}}}{2 \mathrm{k}} \int_{\mathrm{K}<\mathrm{k}}\left[\left|\underline{\mathrm{b}}_{\mathrm{q}}(\underline{\mathrm{K}})\right|^{2}-\left[\left.\underline{\mathrm{a}}_{\mathrm{q}}(\underline{\mathrm{K}})\right|^{2}\right] \gamma(\mathrm{K}) \mathrm{dK},\right.
$$

where $\underline{b}_{q}(\underline{K})$ and $\underline{a}_{q}(\underline{K})$ are the "complete" spectral vectors defined in $(1.2-12)$. This form is more convenient in some cases.

Example 1.4-2: Consider an antenna operating in a transmitting mode, no spatial waves being incident. Show that time-average power radiated per

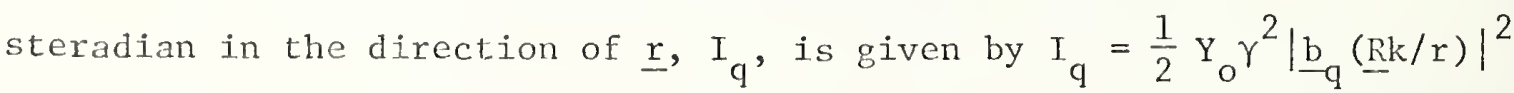
(properly normalized, this expression leads directly to the power-gain formula (1.6-6)). Total time-average power radiated into a hemisphere, ${ }_{\mathrm{q}}$, is given by $\frac{1}{2} \hat{\mathrm{b}} \hat{\mathrm{n}}_{\mathrm{q}} \hat{\mathrm{b}}_{\mathrm{q}}$. Show that

$$
\int \mathrm{I}_{\mathrm{q}} \mathrm{d} \Omega=\mathrm{P}_{\mathrm{q}},
$$


where $\mathrm{d} \Omega$ is the differential of solid angle and the integration goes over the appropriate hemisphere. The integration here is sometimes called "pattern integration" although $I_{q}$ is not merely a pattern function.

Total radiated power, $\mathrm{P}_{\text {rad }}=\mathrm{P}_{1}+\mathrm{P}_{2}$, is involved in the definitions of directivity

$$
\mathrm{D}_{\mathrm{q}} \equiv \mathrm{I}_{\mathrm{q}} / \mathrm{P}_{\mathrm{rad}}
$$

and antenna efficiency

$$
\mathrm{h} \equiv \mathrm{P}_{\mathrm{rad}} / \mathrm{P}_{\mathrm{O}}
$$

where $P_{0}$ is net input power to the antenna at $S_{0}$. For a lossless antenna $h=1$ and (12) becomes a conservation relation which can be written

$$
\sum_{q} \int_{K<k} \sum_{m}\left|S_{q o}(m, \underline{K})\right|^{2} \eta_{m}(K) d \underline{K}=\eta_{o}\left(1-\left|S_{o o}\right|^{2}\right)
$$

Consequences of this constraint on the parameters of a lossless antenna will be noted in Chapter III.

More complete consideration of energy distribution, as for example in the "optical theorem" and in the theory of minimum scattering antennas, as well as in concepts of scattering crosssections, necessarily involves one with the concept of "scattered power." This we define and discuss as follows.

Consider a scattering object with a field (from remote sources) incident from the left and with no field incident from the right; i.e., $\hat{a}_{2} \neq 0, \hat{a}_{1}=0$. The concept of scattered power in the circumstances considered and in our notation is expressed by

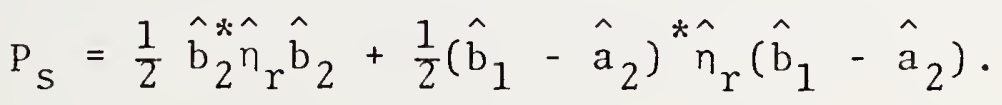


The first term on the right gives the cis-scattered power and is unexceptionable, because of the complete power orthogonality between incoming propagated waves and outgoing waves. The second term is a power expression associated with what we have called bona-fide trans-scattering (into the positive hemisphere). Essentially the expression (14) is often used as a definition of scattered power without critical comment. Thus, Stratton [29], p. 568, states "... [the] term obviously measures the outward flow of the secondary or scattered energy from the diffracting [object]...". Nevertheless, critical comment is called for. Thus, Silver [45], p. 43, says in essence, "Although electromagnetic fields in space are additive, the energies of [coherent] fields are not additive. Consequently, energy flux calculated for the scattered waves regarded as isolated from the incident waves, does not necessarily represent energy removed from the latter and scattered in all directions." Our comment is that, as long as the definition is used consistently, one may expect consistent mathematical results, but the physical interpretation may occasion some difficulties [31]. We adopt the conventional terminology, and will, without further comment, use the phrases "scattered power" and "scattered power density" as names for integrals of the type involved in (14) and for the corresponding Poynting's vector, respectively.

Cross-terms of the type ignored in connection with (14) have a crucial role in the derivation of a theorem relating forwardscattered wave-amplitude to total power scattered and absorbed by a finite scattering object. The theorem in question is well known in optics ([31], p. 653) and has been stated several times 
in electromagnetics; the analogous theorem in quantum mechanics, though of considerable earlier origin, is now sometimes known as the "optical theorem" ([32], p. 866). In our context the scattering object may of course be a passive antenna; if so, the power absorbed by the attached receiver is simply part of the total power absorbed.

Example 1.4-3: The "optical theorem." In the situation prescribed for (14), total power absorbed, $\mathrm{P}_{\mathrm{a}}$, is given without ambiguity by

$$
\begin{aligned}
P_{a} & =-\left(P_{1}+P_{2}\right) \\
& =\frac{1}{2} \hat{a_{2}^{*} \hat{\eta_{r}} \hat{a}_{2}}-\frac{1}{2} \hat{b}_{2}^{* \hat{n}} \hat{b}_{2}-\frac{1}{2} \hat{b_{1}^{*} \hat{\eta_{r}}} \hat{b}_{1} .
\end{aligned}
$$

The sum of the power scattered and the power absorbed, called the "extinction power," is given by

$$
\begin{aligned}
P_{x} & =P_{s}+P_{a} \\
& =\hat{a}_{2}^{* \hat{n}_{r}} \hat{a}_{2}-\frac{1}{2} \hat{b}_{1}^{* \hat{n}} \hat{n}_{r} \hat{a}_{2}-\frac{1}{2} \hat{a}_{2}^{* \hat{n}_{r}} \hat{b}_{1} .
\end{aligned}
$$

From the scattering equations we have $\hat{b}_{1}=\hat{s}_{12} \hat{a}_{2}$; hence

$$
\begin{aligned}
P_{x} & =\frac{1}{2} \hat{a}_{2}^{*}\left(2 \hat{n}_{r}-\hat{s}_{12}^{*} \hat{\eta}_{r}-\hat{n}_{r} \hat{s}_{12}\right) \hat{a}_{2} \\
& =-\operatorname{Re}\left[\hat{a}_{2}^{*} \hat{\eta}_{r}\left(\hat{s}_{12}-\hat{1}\right) \hat{a}_{2}\right]
\end{aligned}
$$

(If the scattering object is a passive antenna, we assume for simplicity that the attached load is non-reflecting.) Note that $\hat{\mathrm{S}}_{12}-\hat{1}$ represents the appropriate bona fide trans-scattering characteristic (cf. remarks following (1.3-14)). We now let the incident electric field be the plane wave $\underline{E}=\underline{E}_{0} \exp (i k z)$, as a locally good approximation to a well-collimated beam. Then the corresponding spectrun is

$$
\sum_{\mathrm{m}} a_{2}(\mathrm{~m}, \underline{\mathrm{K}}) \underline{\underline{K}_{\mathrm{m}}}=2 \pi \underline{E}_{\mathrm{O}} \delta(\underline{\mathrm{K}})
$$


and the extinction power becomes

$$
\mathrm{P}_{\mathrm{X}}=-4 \pi^{2} \mathrm{Y}_{\mathrm{O}} \operatorname{Re}\left\{\overline{\mathrm{E}}_{\mathrm{O}} \cdot\left[\underline{\underline{S}}_{12}(0,0)-\underline{\underline{1}}\right] \cdot \underline{\mathrm{E}}_{\mathrm{O}}\right\}
$$

The scattered electric field $\underline{E}_{S}$ evaluated asymptotically on the z-axis at distance $z$ and the corresponding radiation vector $\underline{F}_{S}$ are related to $\underline{S}_{12}(0,0)$ by

$$
e^{-i k z} \underline{Z E}_{S}=\underline{F}_{S}=-2 \pi i k\left[\underline{\underline{S}}_{12}(0,0)-\underline{\underline{I}}\right] \cdot \underline{E}_{0}
$$

Substitution into the above expression for $\mathrm{p}_{\mathrm{x}}$ leads to the expression of the desired theorem:

$$
\mathrm{P}_{\mathrm{x}}=\frac{2 \pi P}{\mathrm{k}} \mathrm{O} \operatorname{Im}\left(\overline{\mathrm{E}}_{\mathrm{O}} \cdot \underline{\mathrm{F}}_{\mathrm{S}}\right)
$$

Example 1.4-4: For an elementary electric-dipole antenna considered lossless and reciprocal, endowed with minimum scattering properties, and provided with a reflectionless load, the following properties hold. 5 The polarizability of the antenna as a scattering object is

$$
\underline{\underline{\alpha}}=\frac{3 \pi i \varepsilon}{k^{3}} \underline{e}-{ }^{e}-p^{3}
$$

where $e_{-p}$ is a real unit vector in the direction of the dipole. The radiation vector of the forward-scattered electric field is thus

$$
\underline{F}_{S}=\frac{3 i}{4 k} \stackrel{e}{-p t}-\underline{p} \cdot \underline{E}_{0}
$$

where $e_{p t}$ is the transverse part of $\underline{e}_{p}$. Power absorbed is just that delivered to the receiver, and power absorbed is equal to power scattered:

$$
\mathrm{P}_{\mathrm{a}}=\mathrm{P}_{\mathrm{S}} \text {. }
$$
5 These and other properties of elementary dipole antennas are derived using
PWSM methods in Chapter III. 
Further, in this case

$$
P_{a}=\frac{3 \lambda^{2}}{8 \pi} \cdot \frac{Y_{O}}{2}\left|E_{-0} \cdot-p\right|^{2}
$$

Show that these properties and the theorem of example 3 are consistent.

Example 1.4-5: Verify the theorem of example 3 for scattering by a sma11 dielectric sphere. Warning: One needs a more precise result for polarizability than the first approximation usually given. Data needed may be found in Van de Hulst [33], p. 144.

\subsection{Reciprocity Relations - A Summary}

In this section we briefly state recently derived [34] generalized or adjoint reciprocity relations for antennas and scatterers. (The derivation is reproduced in Appendix A.) These relations very readily adapt to the expression of ordinary reciprocity as a special case. We shall also comment on the question of "realizability" of mutually adjoint systems. First we must define mutually adjoint media and systems.

We describe the distribution of material media making up an antenna or scattering structure by means of the constitutive equations.

$$
\underline{D}=\underline{\underline{\varepsilon}} \cdot \underline{E}+\underline{\tau} \cdot \underline{H}, \quad \underline{B}=\underline{\underline{v}} \cdot \underline{E}+\underline{\mu} \cdot \underline{H} \cdot
$$


Here the tensors $\underline{\varepsilon}$ and $\underline{\underline{\mu}}$ have their usual roles; $\underline{\underline{\tau}}$ and $\underline{\underline{v}}$ allow for the description of possible magnetoelectric properties of the medium [35]. 6 (This last bit of generality may provide future benefits and does not appreciably complicate the discussion.) The tensor parameters will of course in general depend upon position within the region of the antenna or scatterer considered; outside this region the set of parameters must reduce nominally to vacuum values: $\underline{\underline{\varepsilon}}=\varepsilon_{0}, \underline{\underline{\mu}}=\mu_{0}, \underline{\underline{\tau}}=\underline{\underline{\nu}}=0$.

In addition to a "given" or "original" system, described by the above equations, we must consider the adjoint system, which is described by the constitutive equations for the adjoint mediun, 7

$$
\underline{D}=\underline{\underline{\tilde{\varepsilon}}} \cdot \underline{E}-\underline{\underline{\tilde{v}}} \cdot \underline{H}, \quad \underline{B}=-\underline{\underline{\tilde{\tau}}} \cdot \underline{E}+\underline{\underline{\tilde{I}}} \cdot \underline{H}, \quad(1.5-2)
$$

where the superposed tilde denotes the transpose, and the tensors $\underline{\underline{\varepsilon}}, \underline{\underline{\tau}}, \underline{\underline{\mu}}$, and $\underline{\underline{\nu}}$ are those of the original system. As the equations show, "adjointness" is a mutual relationship: the adjoint of the adjoint system is the original system.

${ }^{6}$ In some of the recent literature, modia described by equations of the form of (1) are called "bianisotropic." However, optically active media, which have bcen studicd for many ycars [36], satisfy cquations of the above form with scalar parameters and arc not anisotropic at a11. Optically active media incidcntally are reciprocal, and thus could form part of a reciprocal antenua or other devicc.

7Media related by (1) and (2) are called "complementary" media by Kong and cheng [37]. The adjective "adjoint" seems more appropriate both nontcchnica11y and technically: Maxwell's equations for the adjoint system can be written as the mathomatical adjoint of Maxwell's cquations for the original systcm. See Appendix $\Lambda$. 
Since the tensor parameters are essentially arbitrary, we should realize that the "original" system is in no way a theoretically preferred system; the designation is arbitrary but useful.

The concept that a medium may be lossy, lossless, or even "gainy" is familiar. In an inhomogeneous medium these "dissipative properties" will in general change from point to point. In Appendix $B$ dissipative properties are more precisely defined and it is shown that these properties are point-wise identical for mutually adjoint media. In view of the usual connection between dissipative properties and the concept of "realizability," we may say that mutually adjoint media are equally realizable.

Nonreciprocal antennas are most commonly (if not invariably) so because of the use of ferrites subjected to a static magnetic biasing field. In such cases the adjoint antenna can in principle be produced by reversal of the bias field. (We say "in principle" because in general no provision is made for conveniently or precisely accomplishing the bias field reversal.)

The scattering matrix for the adjoint antenna may be and is assumed to be defined with the same basis fields, the same reference surfaces, and altogether in the same way as that for the original antenna. Then, as shown in Appendix A, the following generalized or adjoint reciprocity relations hold between the characteristics of mutually adjoint antennas: For the antennafeed reflection coefficients,

$$
\mathrm{S}_{00}=\mathrm{S}_{00}^{\mathrm{a}}
$$


for the transmitting and the receiving characteristics,

$$
\begin{aligned}
& \eta_{0} S_{o q}^{a}(m, \underline{K})=-\eta_{m}(K) S_{q 0}(m,-\underline{K}), \quad(q=1,2) \\
& \eta_{0} S_{o q}(m, \underline{K})=-\eta_{m}(K) S_{q 0}^{a}(m,-\underline{K}) ; \quad(q=1,2)
\end{aligned}
$$

and for the scattering characteristics,

$\eta_{m}(K) S_{p q}(m, \underline{K} ; n, \underline{L})=\eta_{n}(L) S_{p q}^{a}(n,-\underline{L} ; m,-\underline{K}) . \quad(p=1,2 ; q=1,2)(1.5-5)$

The superscript "a" distinguishes quantities associated with the adjoint antenna. We observe that all the equations hold with $\mathrm{S}$ and $\mathrm{S}^{\mathrm{a}}$ interchanged.

Scattering-matrix elements, such as $\mathrm{S}_{10}(\mathrm{~m}, \underline{K})$ and $\mathrm{S}_{11}(\mathrm{~m}, \underline{\mathrm{K}}, \mathrm{n}, \underline{\mathrm{L}})$, characterize processes associated with a pair of directions: ${ }^{8}$ the direction of an incident wave and a direction of "observation." For the functions mentioned, the direction-pairs are $\underline{n}_{0}, \underline{e}_{k}$ and $\underline{e}_{\ell}, \underline{e}_{k}$, respectively. Reciprocity relates processes associated with two pairs of directions, ${ }^{7}$ obtained by reversing and interchanging the direction of incidence and the direction of observation. See figures 4 and 5 .

8 This is in marked contrast to what is involved when spherical or other nonplanar waves are used to represent the ficlds in space. 
If the constitutive tensors obey the symmetry relations

$$
\underline{\underline{\varepsilon}}=\underline{\underline{\tilde{\varepsilon}}}, \underline{\underline{\mu}}=\underline{\underline{\tilde{\mu}}} \text {, and } \underline{\underline{\tau}}=-\underline{\underline{\underline{\tilde{V}}}} \text {, }
$$

then, as may be seen directly from (1) and (2), the adjoint antenna and the original antenna are identical. In this case,
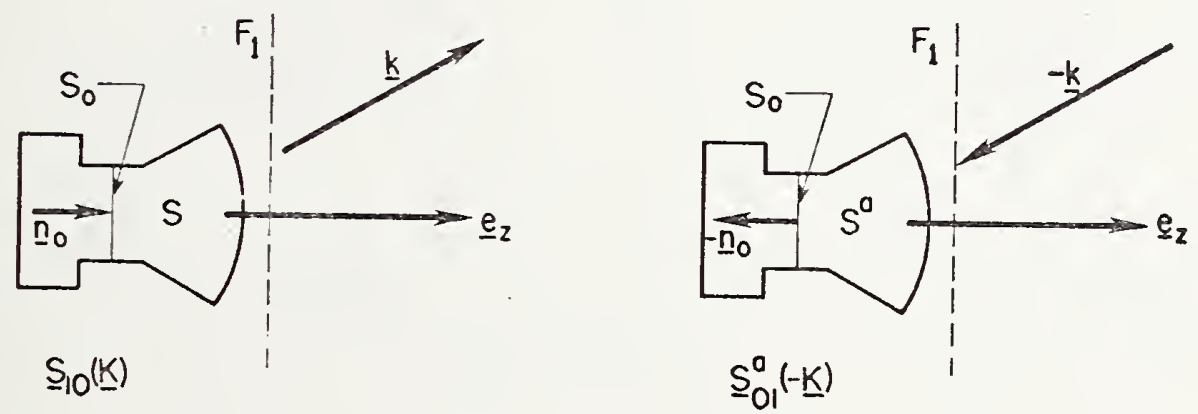

Figure 4. Reciprocity direction-diagram for $\underline{S}_{10}$ and $\underline{S}_{01}^{\text {a }}$.

if we use conventional terminology, we say that the original antenna is reciprocal; in the present context, a term such as self-reciprocal or self-adjoint would be less liable to ambiguity. In the selfadjoint case the superscript "a" is without force and may be eliminated; (3), (4), and (5) become expressions of properties of one and the same antenna (and reduce to results given with $p=q=1$ in [1]). 

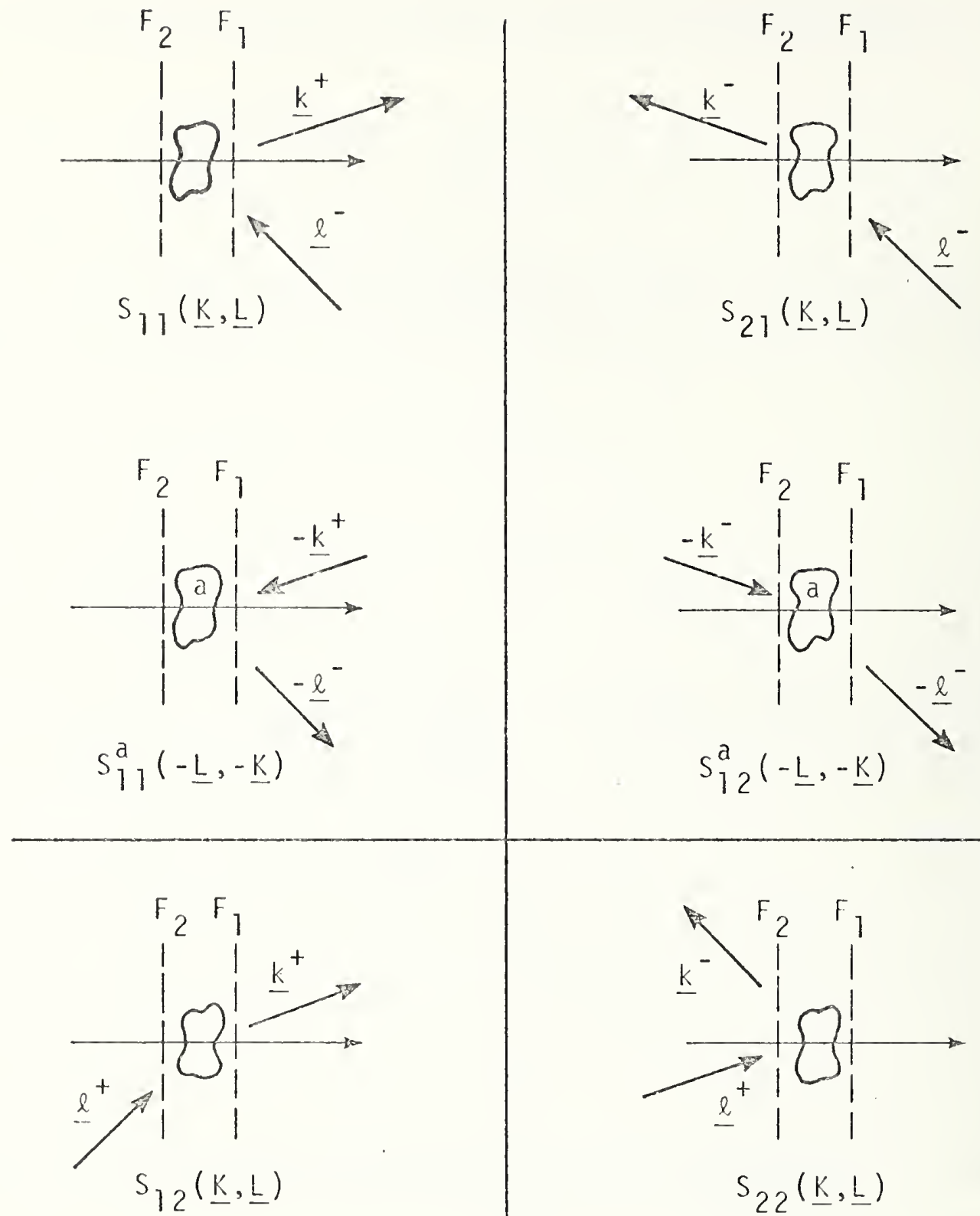

$S_{22}(\underline{K}, \underline{L})$
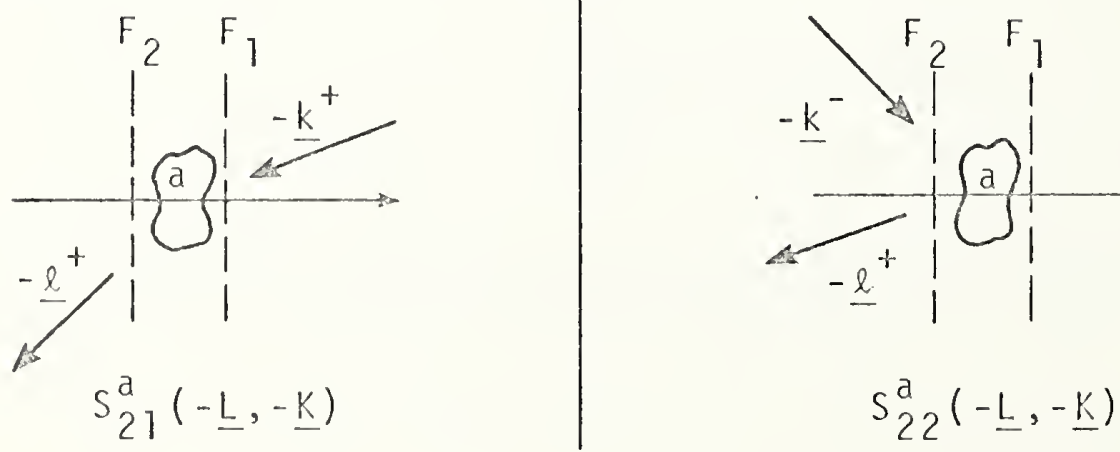

$S_{21}^{a}(-\underline{L},-\underline{K})$

Figure 5. Dircction-diagrams for the scattering reciprocity relations. 
Equations (3), (4), and (5) are our basic expressions of reciprocity and adjoint reciprocity. As mentioned in the Introduction, these relations have found substantial application in research establishing the foundations of the extrapolation technique $[6,7]$. More immediate consequences are brought out in the next subsection and in section 4 . 


\subsection{Relationships between PWSM Quantities and}

Conventional Antenna Quantities

General. Our primary purpose in this subsection is to exhibit some of the content and applicability of the PWSM formalism by defining and expressing some of the more familiar and conventional quantities of antenna theory in terms of antenna scattering-matrix quantities. Our definitions of power gain and effective area (in particular) are consistent with the essential content of the corresponding IEEE Standard definitions. However, our definitions of transmitting and receiving quantitites are (in a11 cases) formed wholly independently of each other--in contrast to a tactic used to some extent in the IEEE Standard definitions and elsewhere. Reciprocity relations then appear only later in a more logical and more substantive role as theorems.

Al1 the quantities to be defined are functions of direction and all are meaningful and applicable in dealing with coupling or scattering at large distances, the context of conventional antenna theory. Although all the quantities to be defined are expressible uniquely in terms of the PWSM quantitics, the converse is true only for the gencralized effective lengths (vectorial quantities) and not for the scalar quantities (such as power gain and polarization indices). The generalized cffective lengths are in fact together almost cquivalent to the PWSM transmitting and receiving characteristics and thus have greater significance than heretofore gencrally recognized. But the scalar quantities (taken singly or in combination) are not adequate characterizations of antenna propertics for the type of theory of essential interest in this monograph. 
Sec. 1.6]

Power gain, effective area, and polarization indices. The key relation appropriate for the present consideration of transmitting characteristics is the asymptotic relation

$$
\underline{E}_{q}(\underline{r}) \sim-i \gamma \underline{s}_{q o}(\underline{R k / r}) a_{0} e^{i k r} / r
$$

Here

$$
\underline{s}_{q 0}(\underline{K}) a_{0}=\underline{b}_{q}(\underline{K})
$$

where $\underline{b}_{q}$ is the complete radiated spectrum in the sense of $(1.2-12 a)$ and $\underline{s}_{q o}$ is the corresponding "complete" transmitting characteristic given in terms of components of $\underline{s}_{q 0}$ by

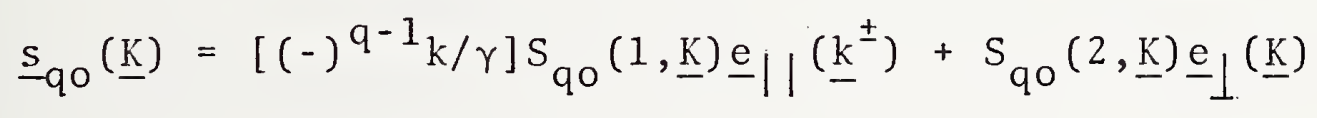

The following relations are noted:

$$
\underline{\mathrm{k}}^{ \pm} \cdot \underline{\mathrm{s}}_{\mathrm{qo}}(\underline{\mathrm{K}})=0, \quad \underline{\mathrm{s}}_{\mathrm{qo}}(0)=\underline{\mathrm{s}}_{\mathrm{qo}_{0}}(0),
$$

and $\underline{S}_{q 0}(\underline{K})$ is the projection of $\underline{s}_{q 0}(\underline{K})$ on the $k_{x}, k_{y}$ plane.

From (1) one easily finds for the power radiated per unit solid angle at large distances (the so-called radiation intensity)

$$
I_{q}(\underline{K})=\left.\left.\frac{1}{2} Y_{0} \gamma^{2}\right|_{q_{0}}(\underline{K}) a_{0}\right|^{2}
$$

The $Y_{0}$ appearing here is the value of the admittance $\left(\varepsilon_{0} / \mu_{0}\right)^{1 / 2}$ for the ambient medium. The function $\mathrm{I}_{\mathrm{q}}$ is a "power pattern" for the considered antenna; generally any function proportional to $\mathrm{I}_{\mathrm{q}}$, whether or not the factor of proportionality is known, is called a power pattern.

The power gain (function) of an antenna is defined by

$$
G_{q}(\underline{K})=4 \pi I_{q} / P_{0},
$$


where $P_{0}$ is the net input power to the antenna. It follows from $(1.1-4)$ and (4) that

$$
G_{q}(K)=\frac{4 \pi Y_{0} \gamma^{2}\left|\underline{s}_{q O}(K)\right|^{2}}{n_{0}\left(1-\left|S_{0 O}\right|^{2}\right)} .
$$

It should be noted that the power gain is a characteristic of the antenna under consideration, independent of the source used to excite the antenna. Furthermore, the value of $\mathrm{G}_{\mathrm{q}}(\mathrm{K})$ is independent of the inscrtion or adjustment of a lossless tuner in the feed waveguide, whether or not this tuner is counted as part of the antema (cf. discussion of $(6.4-4)$ in Chapter 1 ).

Using the components shown in (3), wo define the polarization index

$$
\rho_{q 0}(K)=\frac{S_{q 0}(2, \underline{K})}{S_{q O}(1, \underline{K})(-)^{q-1} k / \gamma}
$$

associated with the transmitting characteristics of an antenna. This spectral polarization index is definable more physically and more conventionally in terms of the components of the corresponding asymptotic E; by (1), it is just the ratio of the $\perp$ and $\mid$ (or $\phi$ and $\theta$ ) components of this $E$ in the direction of obscrvation. polarization characteristics are conveniently and fully described by the single complex number $\rho_{\text {qo }}$; other polarization parameters, such as axial ratio and orientation of the associated polarization cllipse, can be determined, if desired, from $\rho_{q 0}$.

For an antenna functioning in a receiving mode, the counterpart of the power gain is the effective area or cffective receiving cross-section, $\sigma_{q}(K)$. Like the gain, this quantity is a scalar 
function of direction and involves a far-field concept--in this case that of an incident plane wave. It is here defined by

$$
\mathrm{P}_{\mathrm{A}, \max }=\sigma_{\mathrm{q}}(\underline{\mathrm{K}}) \mathrm{S}_{\mathrm{Poy}},
$$

where $P_{A, \max }$ is the available power at the antenna terminal and $S_{\text {Poy }}$ is the magnitude of the Poynting's vector of a plane wave arriving at the antenna from a given direction and providing a polarization match to the antenna receiving characteristic. The derivation of an expression for $\sigma_{\mathrm{q}}$ involves a number of intermediate results that are at least as important as $\sigma_{q}$ itself.

Let the electric field of the wave incident on side $q$ of the antenna be $\underline{E}=\underline{a} \exp (i k \cdot \underline{r}) / 2 \pi$; then from $(1.3-2 a)$ or $(1.3-4 a)$, the emergent wave-amplitude at the antenna terminal is

$$
b_{0}=S_{0 o} a_{0}+\underline{S}_{O q}(\underline{K}) \cdot \underline{A} .
$$

(This expression differs from (1.3-8) only in that here we have not assumed $\left.a_{0}=0.\right)$ Thus, from (9), we see that the antenna, as excited by the incident spatial wave, appears at $S_{0}$ as a source having a reflection coefficient $S_{0 o}$ and a generated wave $\mathrm{b}_{\mathrm{G}}=\underline{\mathrm{S}}_{\mathrm{oq}}(\underline{\mathrm{K}}) \cdot \underline{A}$. By ordinary (microwave) circuit calculation the corresponding received power is found to be

$$
\mathrm{P}_{\text {rec }}=\frac{1}{2} n_{\mathrm{O}} \frac{\left(1-\left|\Gamma_{L}\right|^{2}\right)\left|\underline{S}_{O Q}(\underline{K}) \cdot \underline{A}\right|^{2}}{\left|1-\Gamma_{L} S_{O O}\right|^{2}},
$$

where $\Gamma_{L}$ is the reflection coefficient of the passive termination at $S_{0}$. By setting $\Gamma_{L}=\bar{S}_{00}$, we get for the available power

$$
\mathrm{p}_{\mathrm{A}}=\frac{1}{2} \eta_{0} \frac{\left|\underline{S}_{\mathrm{OQ}}(\underline{K}) \cdot \underline{A}\right|^{2}}{1-\left|\mathrm{S}_{\mathrm{O} O}\right|^{2}}
$$


To aid in the consideration of the polarization-related parts of the problem, we display the transverse spectral-amplitude vector

$$
\underline{A}=a_{1-1} \underline{K}_{1}+a_{2} \underline{K}_{2}
$$

used here and originally defined in (1.2-18); we introduce the complete spectral-amplitude vector for the incident wave in the form

$$
\underline{a}=\left[(-)^{\left.q_{k} / \gamma\right] a_{1}} \underline{e} \mid \underline{(k}^{\bar{\mp}}\right)+a_{2} \underline{e} \underline{L}(\underline{K})
$$

and we define the polarization index

$$
w_{q}(\underline{K}) \equiv \frac{a_{2}}{a_{1}(-)^{q_{k / \gamma}}}
$$

for the incident wave.

Further, it is convenient to define a receiving characteristic, soq, complementary to the complete transmitting characteristic $\underline{s}_{q o}$. The single essential requirement is that

$$
\underline{s}_{o q}(\underline{K}) \cdot \underline{a}=\underline{S}_{o q}(\underline{K}) \cdot \underline{A}
$$

be an identity in $\underline{A}$. This leaves a possible $\underline{e}_{k}$-component of $\underline{s}_{o q}$ undefined; we are in fact free to require

$$
\underline{\mathrm{k}}^{\mp} \cdot \underline{\mathrm{s}}_{\mathrm{oq}}(\underline{\mathrm{K}}) \equiv 0
$$

(Here, as before, the upper sign goes with $q=1$, the lower, with $q=2$.$) Equations (14) and (12) imply$

$$
\underline{s}_{o q}(\underline{K})=\left[(-)^{q} \gamma / k\right] S_{o q}(1, \underline{K}) \underline{e} \|\left(\underline{k}^{\mp}\right)+s_{o q}(2, \underline{K}) \underline{e} \perp(\underline{K}),(1.6-15)
$$

One should note particularly that $\gamma / \mathrm{k}$ appears here whereas $\mathrm{k} / \gamma$ appears in the expression for $\underline{s}_{q 0}$; $\underline{s}_{o q}$ is in no way a "complete" vector of which $\underline{S}_{\mathrm{oq}}$ is a part. However, the relation 
Sec. 1.6]

$$
\underline{s}_{o q}(0)=\underline{s}_{o q}(0)
$$

does hold.

Using the components shown in (15), we define a polarization index for the receiving characteristics

$$
\rho_{o q}(\underline{K}) \equiv \frac{s_{o q}(2, \underline{K})}{s_{o q}(1, \underline{K})(-)^{q_{\gamma / k}}} .
$$

This parameter relates to the properties of a passive material structure; it does not directly characterize the elliptical path of a time-varying vector. It is a ratio of receptivities to components of polarization in an incident plane wave under the specified conditions.

Observing that $|\underline{a}|^{2}=8 \pi^{2} \mathrm{~S}_{\mathrm{Poy}} / Y_{\mathrm{O}}$, we can now combine (11), (14a), (13), and (16) to obtain

$$
P_{a}=\left(\frac{\left|1+w_{q} \rho_{o q}\right|^{2}}{\left(1+\left|w_{q}\right|^{2}\right)\left(1+\left|\rho_{o q}\right|^{2}\right)}\right) \frac{4 \pi^{2} n_{o}\left|\underline{s}_{o q}\right|^{2} S_{P_{o y}}}{Y_{o}\left(1-\left|S_{o o}\right|^{2}\right)} .
$$

The quantity in brackets is a "polarization mismatch" factor, which according to the Schwarz inequality for complex vectors, attain its maximum value of unity for

$$
w_{q}(\underline{K})=\overline{\rho_{o q}(\underline{K})}
$$

Thus the condition for polarization match is expressed as a conjugate match of polarization indices. When (18) holds, we find from (17) and (8)

$$
\sigma_{q}(\underline{K})=\frac{4 \pi^{2} n_{O}\left|\underline{s}_{O q}(\underline{K})\right|^{2}}{Y_{O}\left(1-\left|S_{O O}\right|^{2}\right)}
$$

as the desired expression for the effective area. 
The minimum value of $\mathrm{P}_{\mathrm{A}}$, incidentally, is zero: for the given antenna and for any given direction of the incident wave, there is always a wave-polarization, $\mathrm{w}_{\mathrm{q}}=-1 / \mathrm{\rho}_{\mathrm{oq}}$, that is not received at a11. The polarizations best received and not received are mutual1y orthogonal (in the power or Hermitian sense).

The complete transmitting characteristic $\underline{s}_{q o}$ and the complementary receiving characteristic $\underline{s}_{0 q}$ for a reciprocal antenna satisfy the reciprocity relation

$$
\eta_{0} k \underline{s}_{o q}(\underline{K})=Y_{0} \gamma \underline{s}_{q o}(-\underline{K})
$$

which follows from the basic relations (1.5-4). (In applying (1.5-4) it may be convenient to use the forms $\eta_{1}=Y_{0} k / \gamma$ and $\eta_{2}=Y_{0} \gamma / k$ for the n's, which were originally defined in $(1.2-5,-6)$.$) For mutually$ adjoint antennas, the relations corresponding to (20a) are

$$
\eta_{0} k \underline{s}_{O q}^{a}(\underline{K})=Y_{0}^{\gamma} \underline{s}_{q o}(-\underline{K}), \quad \eta_{0} k \underline{s}_{o q}(\underline{K})=Y_{0}^{\gamma} \underline{s}_{q o}^{a}(-\underline{K}) \cdot(1.6-20 b)
$$

The power-gain and effective-area functions for a reciprccal antenna satisfy the well-known reciprocity relation

$$
\sigma_{q}(\underline{K})=\frac{\lambda^{2}}{4 \pi} G_{q}(-\underline{K})
$$

where $\lambda$ is the wavelength defined by $2 \pi / k$. In contrast to derivations by conventional means (Kraus [56], p. 53; Ho11 is et a1. [40]), no specific instance is required to establish the value of the proportionality constant relating $\sigma_{q}$ and $G_{q}$. A similar result, sharing this feature, was derived (apparently for lossless antennas with linearly polarized radiating characteristics) by Brown [20]. For mutually adjoint antennas the corresponding relations are 


$$
\sigma_{q}^{a}(\underline{K})=\frac{\lambda^{2}}{4 \pi} G_{q}(-\underline{K}), \quad \sigma_{q}(\underline{K})=\frac{\lambda^{2}}{4 \pi} G_{q}^{a}(-\underline{K}) .
$$

The results in this set are conveniently found as corollaries of (20). Relations of the type (21b) were noted by Harrington and Villeneuve for antennas containing gyrotropic media [38].

The reciprocity constraint for the transmitting and receiving polarization characteristics of a reciprocal antenna reads

$$
\rho_{\mathrm{oq}}(-\underline{K})=-\rho_{\mathrm{qo}}(\underline{K}) .
$$

The corresponding relations for mutually adjoint antennas are

$$
\rho_{\mathrm{oq}}^{\mathrm{a}}(-\underline{K})=-\rho_{\mathrm{qo}}(\underline{K}), \quad \rho_{\mathrm{oq}}(-\underline{K})=-\rho_{\mathrm{qo}}^{\mathrm{a}}(\underline{K}),
$$

which follow as further consequences of (1.5-4) or (20).

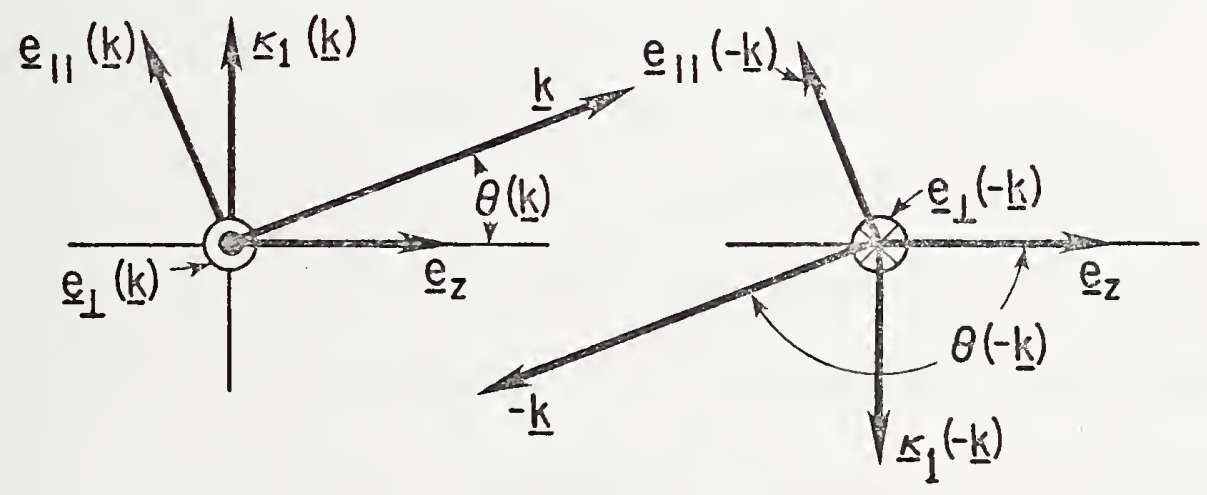

Figure 6. Unit vectors in and perpendicular to the plane of $\underline{\mathrm{e}}_{z}$ and $\underline{k}$ for $\pm \underline{k}, \underline{k}$ reai.

An interesting corollary of the polarization matching and reciprocity theorems is that if the radiation from a reciprocal antenna in a certain direction is circularly polarized, the wave best received from that direction is circularly polarized in the same screw sense. A verification of this, though basicaily simple, involves a few key elements. The polarization-index definitions (7) and (13) are so fashioned that $\rho_{q o}(\underline{K})=i$ and $w_{q}(\underline{K})=i$ both 
represent righthanded circular polarizations ${ }^{9}$ (for $q=1,2$ and for al1 $\mathrm{K}<\mathrm{k}$ ). Here the behavior, and in particular the parity, of the unit vectors $\underline{e} \|$ and $e_{\perp}$ as functions of $\underline{k}$ is directly involved (refer to table 1 and especially to figure 6). Note that $\underline{e}_{1}$, e and $\underline{e}_{k}$ form a right handed system congruent to $\underline{e}_{x}, \underline{e}_{y}$, and $\underline{e}_{z}$. The stated corollary follows upon setting $\rho_{\mathrm{qo}}(\underline{K})= \pm i$ and applying (22a) and (18).

scattering cross-section. The scattering properties of a passive antenna or other scattering object are often conveniently (but not fully) described with the aid of a scalar parameter, $\sigma_{p q}(\underline{K}, \underline{L})$, called briefly "the scattering cross-section." This quantity may be more fully identified as the differential, bistatic, radar scattering cross-section and is defined as follows. Let a plane wave travelling in the direction of the (rea1) propagation vector $\underline{\ell}$ be incident on the scattering object. Let $I_{p}(\underline{K})$ denote the radiation intensity (power radiated per steradian) of the scattered field observed at a large distance in the direction of the propagation vector $\underline{k}$. Then $\sigma_{p q}(\underline{K}, \underline{L})$ is defined by

$$
\sigma_{p q}(\underline{K}, \underline{L}) \equiv 4 \pi \frac{I_{p}(\underline{K})}{S_{\text {Poy }}},
$$

where $S_{\text {Poy }}$ is the magnitude of the Poynting's vector of the incident wave. ${ }^{10}$ This scattering cross-section may of course be expressed in terms of the scattering-matrix elements $\underline{\underline{S}}_{\mathrm{p} q}(\underline{K}, \underline{L})$. The general bistatic $\sigma_{\mathrm{pq}}$ is, however, much more conveniently expressed in terms

${ }^{9}$ See, e.g., Beckmann [39] or Hollis et al. [40]. For optical terminology (which differs) see, e.g., Born and Wolf [31, p. 27].

${ }^{10}$ Equation (23) represents what might be called an "unrationalized" definition, since it contains an effectively superfluous $4 \pi$ (as does the antenna gain definition). This is in accordance with IEEE Standards. A "rationalized" definition, not containing the extraneous $4 \pi$, is often used for scattering processes in physics (including electrodynamics; see, e.g., Jackson [56], p. 489). 
of the biplanar scattering characteristics $t_{p q}$ defined in subsection III-2.2, and the general expression is in fact given in example III-2.2-6. In the paragraph immediate1y following, we give brief consideration to the frequently occurring case of (strict) backscattering, taken on-axis for simplicity.

Example 1.6-1: The on-axis backscattering cross-section, $\sigma_{22}(0,0)$, of a large, perfectly-conducting, rectangular plate of dimensions a $\mathrm{x} b$, set normal to the $z$-axis, is in the physical optics approximation found to be $4 \pi(a b / \lambda)^{2}$.

Methods used earlier in this subsection for the calculation of radiated and incident power densities may be adapted to the evaluation of scattering cross-section. One finds that the definition (23) implies the expression

$$
\sigma_{22}(0,0)=16 \pi^{3} k^{2}\left|\underline{S}{ }_{22}(0,0) \cdot \underline{\alpha}\right|^{2} /|\underline{\alpha}|^{2},
$$

where $\underline{\alpha}$ is the spectral amplitude vector of the incident plane wave. Evident1y scattering cross-section depends upon the polarization of the incident wave as well as upon the properties of the scatterer itself. For a given, fixed scatterer and arbitrarily variable polarization, the range of variation of the scattering cross-section is indicated by the following inequalities.

$$
0<\Lambda_{\min }<\left|\underline{S}_{22}(0,0) \cdot \underline{\alpha}\right|^{2} /|\underline{\alpha}|^{2}<\Lambda_{\max }<M \cdot(1.6-25)
$$

Here $\Lambda_{\min }$ and $\Lambda_{\max }$ are respectively the smaller and the larger of the two eigenvalues of the positive-definite or positive-semidefinite Hermitian dyadic $\underline{\underline{S}}_{22}^{*}(0,0) \cdot \underline{\underline{S}}_{22}(0,0)$ and $M \equiv \sum_{m, n}\left|S_{22}(0, m ; 0, n)\right|^{2}$. 
The inner inequalities in (25) are well known in matrix theory (see e.g., Mirsky [52], p. 388). In the semidefinite case we have

$$
\operatorname{det}\left(\underline{S}_{22}(0,0)\right)=0, \quad \Lambda_{\min }=0, \quad \Lambda_{\max }=M .
$$

Any one of these equalities implies the other two.

Generalized ejfective lengths. The generalized effective length, $\underline{h}_{t}$, for the transmitting mode of an antenna is defined by

$$
\underline{h}_{t}(\underline{k})=\frac{4 \pi i Y_{0}}{k I_{0}} \lim _{r \rightarrow \infty}\left[r e^{-i k r} \underline{E}_{q}(\underline{r})\right]
$$

where $\underline{k}=\underline{r k} / \mathrm{r}$ and $I_{0}$ is the modal "current" in the feed waveguide related to $a_{0}$ and $b_{0} b y$

$$
I_{0}=n_{0}\left(a_{0}-b_{0}\right)=n_{0} a_{0}\left(1-s_{00}\right) .
$$

Equation (27) represents essentially the most general definition of effective length in the literature; cf. Collin and Zucker [57], p. 105, for a brief discussion and a number of further references. By comparison of (27) and (1) we find

$$
\underline{h}_{t}(\underline{k})=\frac{4 \pi Y_{0} \gamma}{\left(1-S_{0 O}\right) n_{0} k} \underline{s}_{q 0}(\underline{K}) .
$$

This equation shows that $\underline{h}_{t}(\underline{k}) / \gamma$ (not $\underline{h}_{t}$ itself) is essentially equivalent to $\underline{s}_{q o}(\underline{K})$. Both $(28)$ and $(27 \mathrm{a})$ require that

$$
\underline{h}_{t}(\underline{k}) \cdot \underline{k}=0
$$


Certainly equation (27a) does not define $\underline{h}_{t}$ when $K>k$, but (28) may be taken to provide an extension of the basic definition to complex $\underline{\mathrm{k}}$.

The generalized effective length, $\underline{h}_{r}$, for the receiving mode of an antenna is defined to have the basic property

$$
V_{\text {oc }} \equiv \underline{h}_{r}(\underline{k}) \cdot \underline{E}_{q} \text {, }
$$

where $V_{o c}$ is the modal "open-circuit voltage" produced in the feed waveguide by an arbitrary incident plane wave with propagation vector $\underline{k}$ and electric-field vector $\underline{E}_{q}$ (evanescent waves included); $V_{O C}$ is given by $V_{O C}=a_{0}+b_{0}$ with $a_{0}=b_{0}$. Equation (30a) leaves a possible $\underline{e}_{k}$-component of $\underline{h}_{r}(\underline{k})$ undefined; to complete the definition we may and do require

$$
\underline{\mathrm{h}}_{\mathrm{r}}(\underline{\mathrm{k}}) \cdot \underline{\mathrm{k}} \equiv 0 \text {. }
$$

To express $\underline{h}_{\mathrm{r}}$ in terms of $\underline{\mathrm{s}}_{\mathrm{oq}}$, we first rewrite (9) using the identity (14a), obtaining

$$
b_{0}=S_{00} a_{0}+\underline{s}_{0 q}(\underline{K}) \cdot \underline{a} .
$$

For a plane wave we have $\underline{a}=2 \pi \underline{E}_{q}$ (as noted earlier); hence we obtain from (31) the expression

$$
V_{O C}=\frac{4 \pi}{1-S_{O O}} \underline{s}_{O q}(\underline{K}) \cdot \underline{E}_{q} \cdot
$$


Comparison of this equation and (30) yields

$$
\underline{h}_{r}(\underline{k})=\frac{4 \pi}{1-S_{O O}} \underline{s}_{O q}(\underline{K})
$$

In this case, in contrast to (28), we do have simple proportionality.

The expression of adjoint reciprocity for $\underline{h}_{t}(\underline{k})$ and $\underline{h}_{r}(\underline{k})$ follows directly from that for $\underline{s}_{q o}$ and $\underline{s}_{o q}$, shown in (20). In fact, considering (33) and (28) for mutually adjoint antennas, we find

$$
\underline{h}_{t}(\underline{k})=\underline{h}_{r}^{a}(-\underline{k}) \text {. }
$$

Note that this equality could not be asserted if we had not imposed (30b). Equation (34) may be taken to hold for $K>k$, consistent with the extended definition of $\underline{h}_{t}$ provided by $(28)$. For a single reciprocal antenna (34) reduces to the simple relation $\underline{h}_{t}(\underline{k})=\underline{h}_{r}(-\underline{k})$ for the effective lengths of the antenna considered.

Example 1.6-2: Generalized effective length $h_{t}$ for a $\lambda / 2$-dipole. From example 2.2-3 in Chapter III we obtain after normalizing with respect to $a_{0}$,

$$
\underline{s}_{q 0}(\underline{K})=\frac{I_{c}}{2 \pi k^{2} Y_{0} a_{o} \gamma} \frac{\cos \left(\pi e_{k} \cdot e_{k} / 2\right)}{I-\left(\underline{e}_{k} \cdot \underline{e}_{p}\right)^{2}} \underline{k} \times\left(\underline{k} \times \underline{e}_{p}\right) .
$$


To simplify this expression we choose $\underline{e}_{z}$ in the direction of $\underline{e}_{p}$, introduce the "polar angle" $\theta$ of $\underline{k}$ with respect to $\underline{e}_{z}$, and use the relation

$$
\underline{k} \times\left(\underline{k} \times \underline{e}_{p}\right)=k^{2} \sin \theta \underline{e} \|(\underline{k}),
$$

which follows from data given in Table 1, page 95. In example 2.2-3, I c represents current at the center of the dipole. If we assume a directlyconnected, zero-length feed transmission-line, we have $I_{c}=I_{0}=n_{o}\left(1-S_{o o}\right) a_{0}$. With the above data, the formula (28) yields the well-known result

$$
\underline{h}_{t}(\underline{k})=\frac{2}{k} \frac{\cos (\pi \cos \theta / 2)}{\sin \theta} \underline{e}_{\|}^{(k)} .
$$

The maximum value of $\left|\underline{h}_{\mathrm{t}}\right|$ for real $\theta$ occurs for $\theta=\pi / 2$ and is equal to $2 \mathrm{~L} / \pi$, where $\mathrm{L}$ is the length of the dipole. 


\section{SCATTERING-MATRIX ANALYSIS OF COUPLED ANTENNAS;} GENERAL SOLUTION FOR SYSTEM 2-PORT

We consider a system consisting of a pair of antenna systems operating in a homogeneous, isotropic, dissipationless medium, as shown in the highly schematic figure 7 . We are primarily interested in this system as a transmission system, with one antenna transmitting and the other receiving. The complete treatment of a transmission system must include effects of scattering by both antennas, and thus automatically includes treatment of reflection systems, in which one antenna functions in both transmitting and receiving modes and the other antenna represents an arbitrary passive (linear) scattering object.

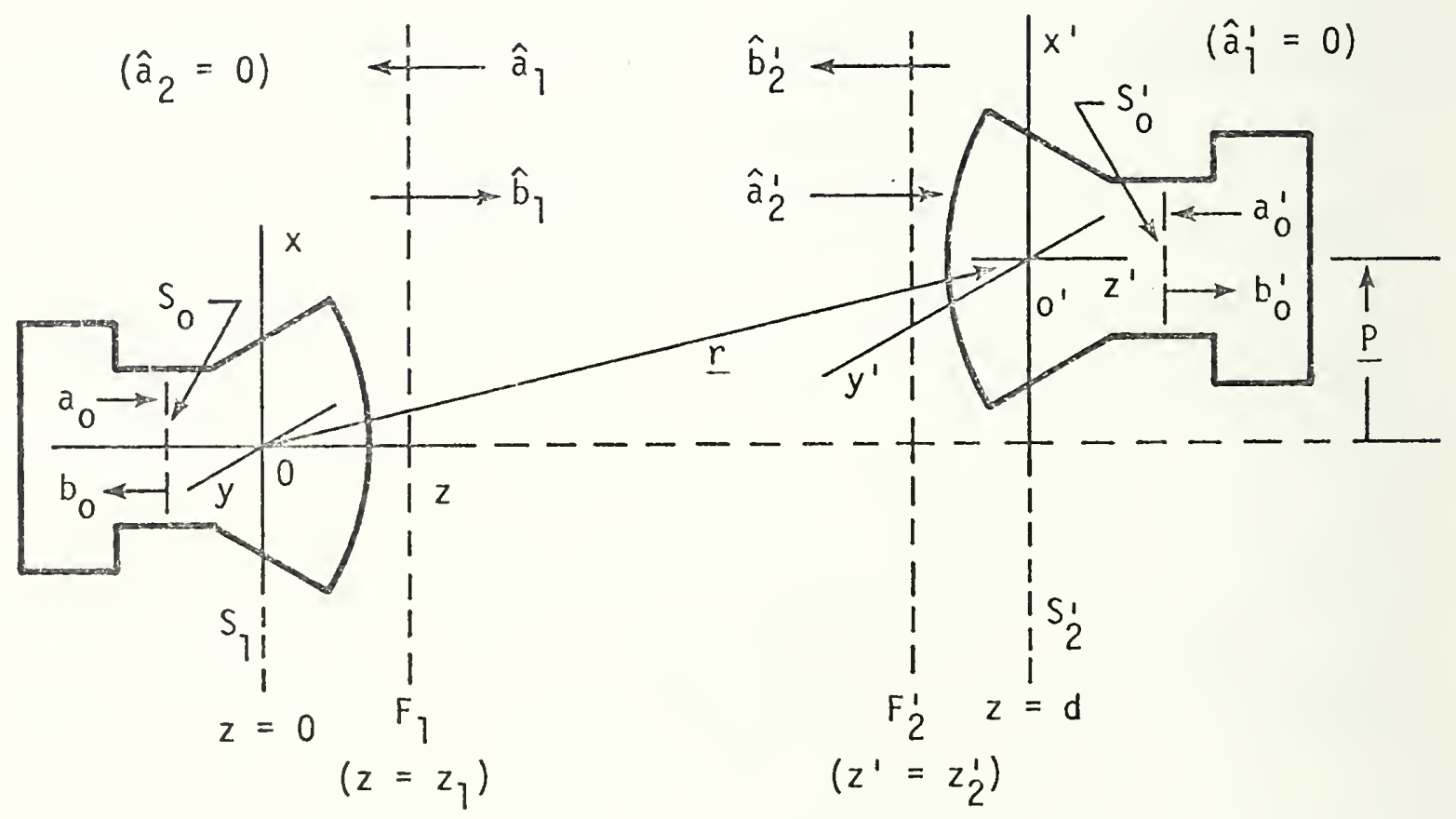

Figure 7. Transmission system--general schematic.

For transverse scanning (subsection 3.1), $d$ is fixed and $\underline{p}$ is variable; in the extrapolation technique (subsection 3.2), $\underline{P}=0$ and $d$ is variable. 
For the description of the antenna on the left in figure 7 , we apply $(1.3-12)$. In the problem of interest there are no waves incident from the left $\left(\hat{a}_{2}=0\right)$; the spectrum of waves going to the $1 \mathrm{eft}, \hat{b}_{2}$, is not involved in the process of solving the problem (but it is obtainable as a part of the solution). Thus (1.3-12) reduces essentially to

$$
\begin{aligned}
& \mathrm{b}_{0}=\mathrm{S}_{00} \mathrm{a}_{0}+\hat{\mathrm{S}}_{01} \hat{a}_{1}, \\
& \hat{b}_{1}=\hat{S}_{10} \mathrm{a}_{0}+\hat{S}_{11} \hat{a}_{1} .
\end{aligned}
$$

These equations are set up with reference to the terminal surface $S_{0}$ and the coordinate system Oxyz. For the description of the antenna on the right, we again apply (1.3-12), using primes to distinguish quantities associated with this antenna. In the problem of interest there are no waves incident on the right side of this antenna $\left(\hat{a}_{i}=0\right)$ and the spectrum of waves going to the right, $\hat{b}_{i}$, is obtainable as a part of the solution. The needed scattering equations thus are

$$
\begin{aligned}
& b_{0}^{\prime}=S_{00}^{1} a_{0}^{\prime}+\hat{S}_{02}^{\prime} \hat{a}_{2}^{\prime}, \\
& \hat{b}_{2}^{\prime}=\hat{S}_{20}^{1} a_{0}^{\prime}+\hat{S}_{22}^{\prime} \hat{a}_{2}^{\prime} .
\end{aligned}
$$

These equations are set up with reference to the terminal surface $S_{0}^{\prime}$ and the coordinate system $0^{\prime} x^{\prime} y^{\prime} z^{\prime}$, which is parallel to the unprimed system and displaced from it by the vector $\underline{r}=\underline{p}+\underline{d e}_{z}$, $\mathrm{d}>0$. Having chosen $\mathrm{d}>0$, we must in fact have $\mathrm{d}>\mathrm{z}_{1}-\mathrm{z}_{2}^{\prime}$, for otherwise the problem is outside the intended scope of our formulation (see subsection 1.2 and especially subsection III-2.2). This constraint we call the "no encroachment" restriction. 
The displacement of the reference coordinate systems for the two antennas implies the joining equations

$$
a_{2}^{\prime}(m, \underline{K})=b_{1}(m, \underline{K}) e^{i \underline{k} \cdot \underline{r}}, \quad a_{1}(m, \underline{K})=b_{2}^{\prime}(m, \underline{K}) e^{-i \underline{k}}-\underline{r},
$$

as shown in appendix $C$. Now, with respect to the transmission path as an element of the system, the set of incident waves is represented by $\hat{b}_{1}, \hat{b}_{2}^{\prime}$ and the set of emergent waves by $\hat{a}_{1}, \hat{a}_{2}^{\prime}$. Thus from (3) the scattering-matrix description of this element is

$$
\left(\begin{array}{c}
\hat{a}_{1} \\
\hat{a}_{2}^{\prime}
\end{array}\right)=\left(\begin{array}{ll}
\hat{0} & \hat{\mathrm{T}}_{12} \\
\hat{\mathrm{T}}_{21} & \hat{0}
\end{array}\right)\left(\begin{array}{l}
\hat{\mathrm{b}}_{1} \\
\hat{\mathrm{b}}_{2}^{\prime}
\end{array}\right)
$$

where the elements of $\hat{\mathrm{T}}_{12}$ and $\hat{\mathrm{T}}_{21}$ are $\mathrm{T}_{12}(\mathrm{~m}, \underline{\mathrm{K}} ; \underline{\mathrm{n}}, \underline{\mathrm{L}})=$ $\delta_{m n} \delta(\underline{K}-\underline{L}) \exp \left(-i \underline{k}^{-} \cdot \underline{r}\right)$ and $\mathrm{T}_{21}(\mathrm{~m}, \underline{K} ; \mathrm{n}, \underline{\mathrm{L}})=\delta_{\mathrm{mn}} \delta(\underline{K}-\underline{\mathrm{L}}) \exp \left(i \underline{\mathrm{k}}{ }^{+} \cdot \underline{\mathrm{r}}\right)$. Equation 4 is obviously equivalent to the two transformations

$$
\hat{a}_{1}=\hat{T}_{12} \hat{b}_{2}^{\prime}, \quad \hat{a}_{2}^{\prime}=\hat{T}_{21} \hat{b}_{1} \text {. }
$$

We are now in a position to obtain a complete formal solution for the behavior of the transmission system under consideration. That is, we can obtain expressions for both $b_{0}$ and $b_{o}^{\prime}$, valid at arbitrary distances and including the effects of multipie reflections. (We can also formally determine the field in the transmission path.) We first consider transmission from left to right, assuming that the receiving antenna is terminated with a passive, reflectionless load. Using (2) and (5), we find

$$
\hat{a}_{1}=\hat{T}_{12} \hat{b}_{2}^{\prime}=\hat{T}_{12} \hat{S}_{22}^{\prime} \hat{a}_{2}^{\prime}=\hat{T}_{12} \hat{S}_{22}^{\prime} \hat{T}_{21} \hat{b}_{1} \equiv \hat{R}^{\prime} \hat{b}_{1} \text {. }
$$


The operator $\hat{\mathrm{R}}^{\prime}=\hat{\mathrm{T}}_{12} \hat{\mathrm{S}}_{22}^{\prime} \hat{\mathrm{T}}_{21}$ defined here is the description of the receiving system, as a passive scattering object, transformed to the reference coordinate system of the transmitting antenna. Substituting (6) in (1) we obtain

$$
\hat{\mathrm{b}}_{1}=\hat{\mathrm{S}}_{10} \mathrm{a}_{0}+\hat{\mathrm{S}}_{11} \hat{\mathrm{R}}^{\prime} \hat{\mathrm{b}}_{1}
$$

which (at least when written out more fully) is seen to be an integral equation determining $\hat{b}_{1}$. (It may be identified as an inhomogeneous, linear integral equation of the second kind.) The solution may be indicated formally by writing

$$
\hat{b}_{1}=\left(\hat{1}-\hat{S}_{11} \hat{R}^{\prime}\right)^{-1} \hat{S}_{10} a_{0}
$$

This gives us the spectrum of outgoing waves in the transmission path; it includes both the simple plane waves and the evanescent waves. $\left(\hat{a}_{1}\right.$ is now determined by $(6) ; \underline{E}_{1 t}(\underline{r})$ and $\underline{H}_{1 t}(\underline{r})$ are determined by (1.2-8).) We may obtain a more explicit but still formal solution to the basic integral equation by the Liouville-Neumann method of successive substitutions. This leads to a representation of the inverse operator in (8) in a series of iterated operators, ${ }^{11}$ so that

$$
\hat{b}_{1}=\left[\hat{1}+\hat{S}_{11} \hat{R}^{\prime}+\left(\hat{S}_{11} \hat{R}^{\prime}\right)^{2}+\cdots\right] \hat{S}_{10} a_{0} \text {. }
$$

The special virtue of this form is that the successive terms in the series correspond to successive round-trip multiple reflections between the transmitting and the receiving antennas. Of course, $\overline{11}$ The operator expansion in (9) is analogous to the finite-dimensional matrix expansion $(1-A)^{-1}=1+A+A^{2}+A^{3}+\ldots$, which is valid if all the eigenvalues of $A$ are less than unity in magnitude. 
(9) is meaningful as an infinite series only if it converges in some useful sense. The domain of convergence will depend upon the "smallness" of the product $\hat{S}_{11} \hat{R}$ ", and it is worth noting that this product depends upon both $\hat{S}_{11}$ and $\hat{S}_{22}^{\prime}$ (as well as upon the distance between the transducers).

We complete this analysis by calculating the scattering matrix of the "system 2-port," which has its terminals at $S_{0}$ and $S_{0}^{\prime}$ and is defined by the equations

$$
\begin{aligned}
& b_{0}=M_{0 O} a_{0}+M_{00^{\prime}} a_{0}^{\prime}, \\
& b_{0}^{\prime}=M_{0} 0_{0}^{a}+M_{0} 0^{\prime} a_{0}^{\prime} .
\end{aligned}
$$

(The properties of a transmission system are often conveniently embodied in this form.) Inasmuch as we have made $a_{0}^{\prime}=0$, solving for $b_{0} / a_{0}$ and for $b_{0}^{1 / a_{0}}$ yields directly

$$
\begin{aligned}
& M_{00}=S_{00}+\hat{S}_{01} \hat{R}^{\prime}\left(1-\hat{S}_{11} \hat{R}^{\prime}\right)^{-1} \hat{S}_{10}, \\
& M_{O^{\prime} O}=\hat{S}_{02}^{\prime} \hat{T}_{21}\left(1-\hat{S}_{11} \hat{R}^{\prime}\right)^{-1} \hat{S}_{10} .
\end{aligned}
$$

A similar alternative solution with $a_{0}=0$ and $a_{0}^{\prime} \neq 0$ yields

$$
\begin{aligned}
& M_{O^{\prime} O^{\prime}}=S_{O O}^{\prime}+\hat{S}_{O 2}^{\prime} \hat{R}_{\left(1-\hat{S}_{22}^{\prime}\right.} \hat{R}^{-1} \hat{S}_{20}^{\prime} \\
& M_{O O^{\prime}}=\hat{S}_{01} \hat{T}_{12}\left(1-\hat{S}_{22}^{\prime} \hat{R}^{-1} \hat{S}_{20}^{\prime},\right.
\end{aligned}
$$

where $\hat{\mathrm{R}}=\hat{\mathrm{T}}_{21} \hat{\mathrm{S}}_{11} \hat{\mathrm{T}}_{12}$. Formulas of this type were first given in [4]; formaly identical expressions are obtained in the electroacoustics case [3]. Their general significance was mentioned in the Introduction. Complete analytical solutions can be found in a highly specialized and idealized (but nevertheless interesting) class of 
problems; in one such solution the associated Liouville-Neumann series is incidentally found to converge or diverge according as $k d$ is greater or less than $0.87993310 \ldots$ (see example III-4-4).

In the measurement technique to be described in subsection 3.1 (but not that in 3.2) we assume that the effects of reflections between antennas have been minimized and may be neglected. When such reflections are omitted, (12) and (13) become $\mathrm{M}_{0^{\prime} \mathrm{O}}=\hat{\mathrm{S}}_{022_{21}^{\prime}} \hat{\mathrm{S}}_{10}$ and $\mathrm{M}_{0^{\prime} \mathrm{O}^{\prime}}=\mathrm{S}_{\mathrm{OO}^{\prime}}^{\prime}$, respectively. If the (passive) termination on the receiving antenna has reflection coefficient $\Gamma_{L}$, we obtain from (10)

$$
b_{0}^{\prime}=F^{\prime} \hat{S}_{02}^{\prime} \hat{T}_{21} \hat{S}_{10} a_{0}^{\prime}
$$

where $F^{\prime}=\left(1-\Gamma_{L} S_{o o}^{\prime}\right)^{-1}$. More explicitly, we have

$$
b_{0}^{\prime}=F^{\prime} a_{0} \int \sum S_{02}^{\prime}(m, \underline{K}) S_{10}(m, \underline{K}) e^{i \underline{k} \cdot \underline{r}} d \underline{K} .
$$

With $\underline{\mathrm{p}}=0$ (15) is essentially a basic and simple case of (43) or (46) in [1].) The integral appearing in this equation is the transmission integral (for transmission from left to right), and the scalar product in the integrand,

$$
\sum_{\mathrm{m}} \mathrm{S}_{\mathrm{o} 2}^{\prime}(\mathrm{m}, \underline{\mathrm{K}}) \mathrm{S}_{10}(\mathrm{~m}, \underline{\mathrm{K}}) \equiv \underline{S}_{02}^{\prime}(\underline{K}) \cdot \underline{S}_{10}(\underline{K})
$$

is called a coupling product. This marks the emergence of the central quantities involved in the antenna measurement techniques described here. 
Examp1e 2-1: Antennas $\mathrm{A}$ and $\mathrm{A}^{\prime}$ are described by the scattering matrices $S$ and $S^{\prime}$, respectively, referred to the fiducial coordinate systems oxyz and $0^{\prime} x^{\prime} y^{\prime} z^{\prime}$ as in figure 7. Transmission integrals for transmission between the two antennas in opposite directions are

$$
\begin{aligned}
& \Psi_{0^{\prime} 0}(\underline{r})=\hat{S}_{02}^{1_{2}} \hat{\mathrm{T}}_{21} \hat{\mathrm{S}}_{10}=\int \underline{S}_{02}^{\prime}(\underline{\mathrm{K}}) \cdot \underline{\mathrm{S}}_{10}(\underline{\mathrm{K}}) \mathrm{e}^{i \underline{\underline{k}} \cdot \underline{\underline{r}} \mathrm{dK}}, \\
& \Psi_{00^{\prime}}(\underline{r})=\hat{S}_{01} \hat{\mathrm{T}}_{12} \hat{\mathrm{S}}_{20}^{\prime}=\int \underline{\mathrm{S}}_{\mathrm{O} 1}(\underline{\mathrm{K}}) \cdot \underline{S}_{20}^{\prime}(\underline{\mathrm{K}}) \mathrm{e}^{-\mathrm{i} \underline{\underline{k}}-\underline{\underline{r}} \mathrm{dK}} .
\end{aligned}
$$

Show that if each antenna is reciprocal, the basic reciprocity relations imply the reciprocity relation

$$
n_{0} \Psi_{00^{\prime}}(\underline{r})=\eta_{0}^{\prime} \psi_{0}^{\prime}(\underline{r})
$$

for the transmission integrals.

In order to establish some of the content of (15) (but not for present applications) we note that the well-known Friis transmission formula can be derived from the asymptotic form of (15). The derivation is slightly simplified if we assume that the fiducial or reference coordinate systems have been chosen so that both antennas are "on axis." Then $\underline{\mathrm{p}}=0, \underline{r}=\underline{\mathrm{d}}_{\underline{z}}$, and the asymptotic form in question is

$$
b_{0}^{\prime} \sim-2 \pi i k F^{\prime} \underline{S}_{02}^{\prime}(0) \cdot \underline{S}_{10}(0) a_{0} e^{i k d} / d
$$

(Thjs is analytically a version of (1.2-16a) or (1.2-16b) evaluated on-axis.) If we now calculate the ratio of the available power at the receiving antenna terminals to the net power input at the transmitting antenna terminals and use the Schwarz inequality, we obtain

$$
\frac{P_{A}^{\prime}}{P_{0}}<\frac{G_{1}(0) \sigma_{2}^{\prime}(0)}{4 \pi d^{2}},
$$


where $G_{1}(0)$ pertains to the transmitting antenna, $\sigma_{0}^{\prime}(0)$ pertains to the receiving antenna, and we have used $(1.6-6,-19)$. Equality in (18) holds for polarization match.

We discuss very briefly one more important result, contained in (11). The first iterated integral in the Liouville-Neumann series for $M_{00}$ is the reflection integral,

$$
\Phi_{00}(\underline{r})=\int d \underline{K} e^{-i \underline{k}-\underline{r}} \underline{S}_{01}(\underline{K}) \cdot \int \underline{\underline{S}}_{22}^{\prime}(\underline{K}, \underline{L}) \cdot \underline{S}_{10}(\underline{L}) e^{-i \underline{\ell} \cdot \underline{r}} \mathrm{~d} \underline{L} \cdot
$$

This is the simplest form of integral involving a scattering or reflecting process; it gives the modification of the input reflection coefficient of an antenna due to the presence of a scattering object when multiple reflections are negligible. The scattering object may, in particular, be an infinite plane reflecting surface, as in the case of an antenna over a flat, homogeneous earth. If the reflecting surface is perfectly reflecting (and perpendicular to the $z$-axis $)$, then $\underline{\underline{S}}_{22}^{\prime}(\underline{K}, \underline{L})=\left(\underline{e}_{z} \underline{e}_{z}-\underline{\underline{1}}\right) \delta(\underline{K}-\underline{L})$, where $\underline{\underline{1}}$ denotes the unit dyadic, and one obtains the interferometer reflection-integral used in [1]. With a scattering object of finite dimensions, (19) is a basic monostatic radar equation, which is (apart from multiple reflections between target and transceiver) valid at arbitrary distances.

Example 2-2: Using the expressions for plane-wave basis fields set up in subsection 1.2, derive Snell's Iaws and the Fresnel coefficients for reflection and transmission at a plane interface $(z=0$, say) between homogeneous isotropic media. The Fresnel reflection coefficients are, of course, the elements of $\underline{S}_{22}^{1}$ appropriate to the antenna-over-flat-earth problem mentioned above. The expressions are simpler and more immediately useful if one does not introduce the conventional (real or complex) angle $\theta$. 
When the scattering object is of finite size, the reflection integral implies the classical radar equation in much the same way as the transmission integral implies the Friis formula. In fact, if we choose the reference coordinate systems as in (15) and set $\underline{r}=\mathrm{de}_{2}$, the asymptotic form of (19) for large d is

$$
\Phi_{0 O}\left(\underline{d e}_{z}\right) \sim-\left(\frac{2 \pi k}{d}\right)^{2} \underline{S}_{01}(0) \cdot \underline{S}_{2}^{\prime}(0,0) \cdot \underline{S}_{10}(0) e^{2 i k d}
$$

This result is contained in (III-5.2-2). Noting that $\mathrm{b}_{\mathrm{O}}=\mathrm{S}_{\mathrm{OO}_{0}} \mathrm{a}_{\mathrm{O}}$ $+\Phi$ oo $\left(\mathrm{de}_{\mathrm{z}}\right) \mathrm{a}_{\mathrm{O}}$ to the approximation being considered, one finds for the available power returned at the antenna terminal

$$
\mathrm{P}_{\mathrm{A}, \text { ret }}=\frac{\mathrm{J}}{2} \frac{n_{\mathrm{O}}}{1-\left|\mathrm{S}_{\mathrm{O} O}\right|^{2}}\left(\frac{2 \pi \mathrm{k}}{\mathrm{d}}\right)^{4}\left|\underline{\mathrm{S}}_{\mathrm{O} 1}(0) \cdot \underline{\underline{S}}_{22}^{1}(0,0) \cdot \underline{\mathrm{S}}_{1 \mathrm{O}}(0)\right|^{2}\left|\mathrm{a}_{\mathrm{O}}\right|^{2}
$$

Splitting the coupling product between the factors $\underline{S}_{01}$ and $\underline{S}_{2} 2$ and using the Schwarz inequality, we next obtain

$$
P_{A, r e t}<\frac{1}{2} \frac{\eta_{0}}{1-\left|S_{0 O}\right|^{2}}\left(\frac{2 \pi k}{d}\right)^{4}\left|\underline{S}_{01}(0)\right|^{2}\left|\underline{S}_{2} i_{2}(0,0) \cdot \underline{S}_{10}(0)\right|^{2}\left|a_{0}\right|^{2}(2-22)
$$

as an upper bound for (21). Now, the pertinent value of the differential cross-section for on-axis backscattering is obtained from (1.6-24) by replacing $\underline{\alpha}$ in that equation by $\underline{S}_{10}(0)$ (and $\underline{S}_{22}$ by $\underline{S}_{22}^{\prime}$ ). Combining the resulting expression with (22), $(1.6-6),(1.6-19)$ and $(1.1-4)$, we obtain the radar "equation" (actually an inequality) in the form

$$
\frac{P_{A, \text { ret }}}{P_{0}}<\frac{\sigma_{1}(0) \sigma_{22}^{\prime}(0,0) G_{1}(0)}{(4 \pi)^{2} d^{4}}
$$

Equality could be restored by the use of a polarization mismatch factor like that in $(1.6-17)$. 


\section{DETERMINATION OF COUPLING-PRODUCT VALUES}

\subsection{Deconvolution of Transverse Scanning Data; Application} of Sampling Theorem

We can now quite easily give the analytical basis for determining coupling-product values from transmission data taken in a transverse plane. The required relative transverse displacement between the transmitting and the receiving antennas is denoted by a transverse displacement $\underline{p}=x_{-x}+y_{-y}$ of the receiving antenna, as shown in figure 7 . Equation $(2-15)$ is directly applicable; we choose to write the phase $\underline{k} \cdot \underline{r}$ in the form $\underline{K} \cdot \underline{P}+i \gamma d$ and thus have

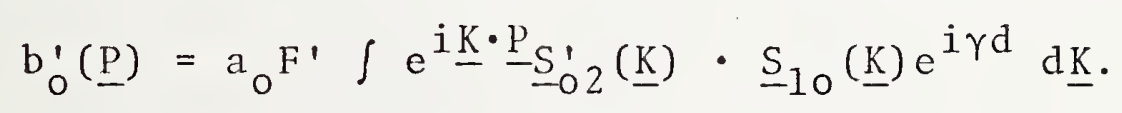

Note that the quantity $b_{0}^{\prime}(\underline{p})$ is what is observed in the measurement process; it will not in general be simply related to the $\underline{E}$ of the transmitting antenna at the point $(\underline{P}, d)$. Indeed, this functional relation may involve derivatives of the components of $\underline{E}$, depending upon the multipole equivalent of the characteristics of the receiving antenna. Thus, in particular, an ideal magnetic dipole (approximated by a loop) receiving antenna would involve those derivatives making up the curl of $\underline{E}$.

Inasmuch as (1) represents a Fourier transformation, its inversion is immediate: We write

$$
\underline{S}_{0}^{\prime}(\underline{K}) \cdot \underline{S}_{10}(\underline{K})=D(\underline{K}),
$$

where $D(\underline{K})$ is here an abbreviation for the determinate function of $\underline{K}$ given by

$$
D(\underline{K}) \equiv \frac{e^{-i \gamma d}}{4 \pi^{2} F^{\prime} a_{0}} \int b_{0}^{\prime}(\underline{P}) e^{-i K} \cdot \underline{P} d \underline{P} .
$$


The inversion of (1) is appropriately termed doconvolution (an explicit convolution form of (1) is shown in Appendix D). The term distinguishes the inversion of (1) from the inversion of the simpler equation (1.3-6) representing the Fourier-transform definition of $\underline{\mathrm{S}}_{\mathrm{qo}}$.

The right-hand side of (3) is in fact determined (up to a phase factor) by the following measurable quantities: the distance d between the reference planes $S_{1}$ and $S_{2}^{\prime}$; the reflection coefficients $\Gamma_{L}$ and $S_{O O}^{\prime}$; and the magnitude and relative phase of $b_{0}^{\prime}(\underline{P}) / a_{0}$ as a function of $\underline{P}$. (For convenience in measurement $b_{0}^{\prime}(\underline{P})$ may be normalized in two stages, indicated by the expression

$$
\frac{b_{0}^{\prime}(\underline{p})}{a_{0}}=\frac{b_{0}^{\prime}\left(\underline{P}_{0}\right)}{a_{0}} \cdot \frac{b_{0}^{\prime}(\underline{p})}{b_{0}^{\prime}\left(\underline{P}_{0}\right)} \text {, }
$$

where $\underline{\mathrm{p}}_{\mathrm{O}}$ is a selected fixed point.)

An instructive hypothetical case in which our analysis correctly shows that no probe correction, other than a known constant multiplicr, would be needed occurs when the receiving antenna is considered to be an ideal electric-field probe. Such a probe can be described and treated analytically as an elementary electric dipole antenna, assumed lossless and reciprocal. For such a probe it can be shown that the recciving response is $b_{0}^{\prime}(\underline{p})=C \underline{E}(\underline{P}) \cdot \underline{e} p$, where $e_{p}$ is a unit vector giving the orientation of the dipole and the absolute value of the constant $C$ can be calculated (see example 2.2-8(e) in Chapter III). Evidently the assumed use of an idcal probe oricnted in the $x$-direction, say, in (3), would effectively reduce (3) to the $\underline{e}_{x}$-component of $(1.3-6)$.

As (1) shows, planar scanning (in the abscnce of multiple reflections) can be interpreted rigorously as a spatially invariant, lincar, filtering process in the two-dimensional wavenumber domain 
(for filtering concepts see e.g., [53]). The concept of the action of a receiving antenna as a filter has been noted, e.g., by Brown [19], apparently with non-planar scanning in mind. The processes are substantially different in the planar and the non-planar cases, as becomes clearly evident upon consideration of the action of a highly directive receiving antenna. Nevertheless, the ability to account fully for the probe characteristics in the planar case suggests the use of a well-characterized "large" probe to reduce data taking and processing effort. Measurements at NBS using such a probe have produced the expected beneficial results. The filtering process has also been studied by Joy and Paris [10] using digital filtering (after data taking) to simulate probe characteristics.

Clearly the utility of the result (3) depends upon one's ability to evaluate the transform of the empirically observed $b_{0}^{\prime}(\underline{p})$. Both least-square fitting and a two-dimensional form of the sampling theorem have been successfully used to evaluate the required transform of $b_{o}^{\prime}(\underline{P})$ from data taken at the points of a rectangular lattice in the measurement plane $[2,8]$. The application of the sampling theorem has become the method of choice, mainly because of the greater ease of computation, and will be described very briefly here.

The essential requirement of the sampling theorem is that the function to be sampled be representable as the Fourier transform of a band-limited function. That $b_{0}^{\prime}(\underline{P})$ virtually fulfills this condition may be seen from (1): a band-1imit $K_{B}$ somewhat greater than $k$, and a distance $d$, may be chosen so that evanescent waves for all $\mathrm{K}>\mathrm{K}_{\mathrm{B}}$ are assuredly virtually zero in the measurement plane (e.g., with $\mathrm{K}_{\mathrm{B}}=1.05 \mathrm{k}$ and $\mathrm{d}=15 \lambda$, attenuation at the band 
limit is approximately $260 \mathrm{~dB}$ ). Bandlimiting within a smaller spectral region may result from the behavior of the product $\underline{S}_{0}^{\prime}(\underline{K}) \cdot \underline{S}_{10}(\underline{K})$ in individual cases (an early study is reported in [8]). If we assign band limits $k_{1} \equiv \pm 2 \pi / \lambda_{1}$ and $k_{2} \equiv \pm 2 \pi / \lambda_{2}$ for $k_{x}$ and $k_{y}$, respectively, a straightforward generalization of the usual one-dimensional theory, given in Appendix 'C, leads to

$$
D(\underline{K})=\frac{e^{-i \gamma d}}{4 k_{1} k_{2} F^{\prime} a_{0}} \sum_{r, s} b_{o}^{\prime}\left(\underline{P}_{r s}\right) e^{-i K} \cdot \underline{P}-r s .
$$

The vectors $\underline{\mathrm{p}}_{\mathrm{rs}}=\frac{1}{2} \mathrm{r} \lambda_{1} \underline{\mathrm{e}}_{\mathrm{x}}+\frac{1}{2} \mathrm{~s} \lambda_{2} \underline{\mathrm{e}}_{\mathrm{y}}$ (with $\mathrm{r}, \mathrm{s}=\ldots-1,0,1$, $2, \ldots)$ define the measurement lattice, the quantitites $b_{0}^{\prime}\left(\underline{P}_{r s}\right)$ are the (complex) values of probe output directly observed at the points of the lattice, and the summation goes over the points of the lattice. According to the sampling theorem, (4) is mathematically exact; that is, if the data [the $\left.b_{0}^{\prime}\left(\underline{P}_{r s}\right)\right]$ were complete and exact, the result would be exact. (Although (4) is exact, it is not the "best possible" result; more advanced theory [42] shows that a rhomboidal lattice would be somewhat more efficient than the rectangular lattice.) The theorem requires an infinite sum, but in the applications thus far we have found that rot even all values measurable above noise are needed.

An important feature of (4) is that the highly efficient algorithm known as the "fast Fourier transform" is rigorously applicable to evaluate the sum.

\subsection{Received Signal as a Function of Distance and the Extrapolation Technique}

The extrapolation technique was introduced by Wacker and Bowman, has been described very briefly by Newell and Kerns [9], and more fully, in an experimentally oriented paper, by Newe11 et a1. 
The theory and numerical techniques were developed by Wacker [6]. The papers mentioned should be consulted for details; in the following paragraphs we give only a brief account of the main ideas and equations involved.

In the extrapolation technique, antenna fiducial coordinates are chosen so that with the antennas in the desired alignment the on-axis $(\underline{p}=0)$ equations apply. Then one observes $b_{0}^{1} / a_{0}$ as a function of antenna separation distance $d$, which is precisely defined by the choice of reference surfaces $S_{1}$ and $S_{2}^{\prime}$ associated with the respective antennas (figure 7 ).

From (2-10) for the system 2-port, we obtain the expression

$$
b_{0}^{\prime}=a_{0} M_{0} O_{0} /\left(1-M_{0} O^{\prime} \Gamma_{L}\right)
$$

which is a precise and complete version of (2-15). Expressions for the elements $M_{O^{\prime}} O_{\text {and }} \mathrm{M}_{O^{\prime} O^{\prime}}$ are given in $(2-12,-13)$, from which, by a rather lengthy process, one finds for (1) as a function of d a series representation of the form

$$
b_{0}^{\prime}=a_{0} F^{\prime} \sum_{p=0}^{\infty} \frac{\exp [i(2 p+1) k d]}{d^{2 p+1}} \sum_{q=0}^{\infty} A_{p q} d^{-q}
$$

Here $F^{\prime}=\left(1-S_{O O}^{\prime} \Gamma_{L}\right)$, and it should be noted that the $A_{p q}$ other than $A_{00}$ will depend on the product $S_{O O}^{\prime} \Gamma_{L}$. We observe that the subseries of terms with a given $\mathrm{p}$ can be interpreted as the contribution of a wave which has experienced $2 \mathrm{p}$ reflections or made $2 p+1$ transits between antennas. In particular, the subseries with $\mathrm{p}=0$ involves no reflections and is the expansion of the transmission integral (2-15): 


$$
\Psi_{O}^{\prime}{ }_{O}(d)=\frac{e^{i k d}}{d}\left(A_{O O}+A_{O L} d^{-1}+A_{O 2} d^{-2}+\ldots\right)
$$

It is of considerable analytical interest that this subseries is not merely asymptotic but actually convergent for sufficiently large d, under the main hypothesis that the two antennas involved be of finite size. If d is measured between centers of spheres, of radii $r$ and $r^{\prime}$, each circumscribing one of the antennas, then $d>d_{0}=r+r^{\prime}$ is sufficient. A sharper, but more complicated, prescription for $\mathrm{d}_{0}$ can be given. See [6], [7], and for more general results, Chapter III of this monograph.

By comparison of (3) and $(2-17)$ we see that

$$
A_{00}=-2 \pi i k \underline{S}_{02}^{\prime}(0) \cdot \underline{S}_{10}(0)
$$

Hence determination of the leading coefficient in (2) is tantamount to the determination of the on-axis value of the spectral coupling product. The basic idea of what we may call the conventional measurement method is simply to have d large enough to make other terms negligible compared to the leading term of the series. The basic idea of the extrapolation technique is to observe $b_{0}^{\prime}$ as a function of $d$ and to fit this function with as many terms of (2) as may be significant, and so to determine a good value for $A_{0 o}$ in particular. This enables one to cope with proximity effects and with multiple reflections between antennas. 


\section{UTILIZATION OF COUPLING-PRODUCT DATA}

\subsection{One Unknown Antenna (Transmitting or Receiving)}

In this subsection we provide basic equations for the use of coupling-product data in a basic antenna measurement situation: the measurement of an unknown antenna (transmitting or receiving) with the requisite known antennas or antenna. Some of the concepts and notation established will be used in the next two subsections (which provide a partial answer to the question, "How does one obtain the first known antenna?").

In this subsection "nothing more" than the algebra of two (complex) linear equations in two (complex) unknowns is involved. The only likely case involving non-uniqueness and compatibility conditions is discussed. (In the following subsections the linear equations have to be reinterpreted as quadratics.)

It will suffice to consider only the case in which the unknown antenna is transmitting; the discussion of the other case would, of course, be analytically very similar.

We do not make simplifying a priori assumptions concerning symmetry or polarization (or other) characteristics of the antenna to be measured. Quite generally, then, we require measurements to be made with (at least in effect) two receiving antennas, $A$ and $B$, having suitable known receiving characteristics, $\underline{A}_{\mathrm{O} 2}(\underline{K})$ and $\underline{B}_{02}(\underline{K})$. From such measurements, the values of the coupling products

$$
\begin{aligned}
& \underline{A}_{02}(\underline{K}) \cdot \underline{S}_{10}(\underline{K})=D_{A}(\underline{K}), \\
& \underline{B}_{02}(\underline{K}) \cdot \underline{S}_{10}(\underline{K})=D_{B}(\underline{K})
\end{aligned}
$$


are to be determined for the desired values of $\underline{K}$. For each chosen, fixed value of the parameter $\underline{K}$, we have two (complex) equations for the two (complex) components of $\underline{S}_{10}(\underline{K})$.

Complete solvability of (1) requires that the vectors $A_{0} 2$ and $\underline{B}_{02}$ be linearly independent at the value of $\underline{K}$ considered. A measure of the linear independence of the two vectors is given by the following expression for the normalized squared-magnitude of the determinant of $(1)$ :

$$
\frac{|\Delta|^{2}}{|\underline{A}|^{2}|\underline{B}|^{2}}=1-\frac{|\underline{A} \cdot \underline{\bar{B}}|^{2}}{|\underline{A}|^{2}|\underline{B}|^{2}}
$$

As the Schwarz inequality shows, this quantity ranges from the value zero when $\underline{B}_{02}$ is proportional to $\underline{A}_{02}$ to a maximum of unity when $\bar{B}_{02} \cdot A_{0} 2$ vanishes. In other words, power orthogonality represents the extreme case of linear independence. Examples are linear polarizations at right angles and left-and right-circular polarizations. An explicit, coordinate-free solution of (1) may be obtained with the use of the set of vectors reciprocal to $\underline{A}_{02}$ and $\underline{B}_{02}$. The reciprocal set $\underline{\alpha}, \underline{\beta}$ is defined by

$$
\underline{\alpha} \cdot \underline{A}_{02}=1, \quad \underline{\alpha} \cdot \underline{B}_{02}=\underline{B} \cdot \underline{A}_{02}=0, \quad \underline{B} \cdot \underline{B}_{02}=1
$$

(assuming the required linear independence), and

$$
\underline{S}_{10}=D_{A} \underline{\alpha}+D_{B} \underline{B}
$$

as is easily verified. The algebra is summed up in the statement that $\mathrm{D}_{\mathrm{A}}$ and $\mathrm{D}_{\mathrm{B}}$ are the covariant components of $\underline{S}_{-10}$ with respect to $\underline{A}_{02}$ and $\underline{B}_{02}$ as base vectors. 
In much of what follows the use of $x, y$ components relative to the fixed basis $\underline{e}_{x}, e_{-}-$- rather than the 1,2 components relative to the variable unit vectors $\underline{\kappa}_{1}, \underline{\kappa}_{2}-$ is indicated. The consideration of geometric rotations and symmetries is appreciably complicated by the dependence of the $\underline{k}$ 's upon $\underline{K}$. Also, the use of $\underline{e}_{x}$, $\underline{e}_{y}$ automatically takes care of the matter of defining the $\underline{k}$ 's on-axis. The required coordinate transformations will be governed by the relationship of the unit vectors

$$
\underline{\kappa}_{1}=\underline{c}_{x}+\underline{s e}_{-}, \quad \underline{\kappa}_{2}=-\underline{s}_{x}+c \underline{e}_{y}
$$

where $c \equiv \cos \phi=\mathrm{k}_{\mathrm{x}} / \mathrm{K}$ and $\mathrm{s} \equiv \sin \phi=\mathrm{k}_{\mathrm{y}} / \mathrm{K}$.

We introduce the abbreviations

$$
A_{x}=A_{02 x}(\underline{K}), \quad B_{x}=B_{02 x}(\underline{K}), \quad S_{x}=S_{10 x}(\underline{K}), \quad(x=x, y)
$$

and write (1) in component form

$$
\begin{aligned}
& A_{x} S_{x}+A_{y} S_{y}=D_{A}, \\
& B_{x} S_{x}+B_{y} S_{y}=D_{B} .
\end{aligned}
$$

Instead of using two intrinsically different antenras $A$ and $B$, in many cases it may be possible and convenient to use one antenna in two orientations, differing by rotation around the $z$-axis by 90 degrees, say in the direction of $x$ to $y$. If antenna $A$ is so used and so rotated, we obtain for the equivalent of antenna $B$

$$
\begin{aligned}
& B_{02 x}\left(k_{x}, k_{y}\right)=-A_{02 y}\left(k_{y},-k_{x}\right) \\
& B_{02 y}\left(k_{x}, k_{y}\right)=A_{02 x}\left(k_{y},-k_{x}\right) .
\end{aligned}
$$


These equations express the rotation of the vector field $\underline{A}_{02}$ corresponding to the rotation of the antenna that it describes. ${ }^{12}$ They lead to a modified version of (6), which we shall discuss in the particularly interesting case of evaluation on-axis $(\underline{K}=0)$. If we let $A_{x}^{O}=A_{o 2 x}(0), S_{x}^{O}=S_{10 x}(0)$, etc., the modified form of $(6)$ is

$$
\begin{aligned}
A_{x}^{O} S_{x}^{O}+A_{y}^{O} S_{y}^{O} & =D_{A}(0), \\
-A_{y}^{O} S_{x}^{O}+A_{x}^{O} S_{y}^{O} & =D_{B}(0) .
\end{aligned}
$$

The determinant of the system (8) vanishes if and only if $A_{x}^{O}= \pm i A_{y}^{O}$; that is, the determinant vanishes if and only if the response characteristic $\underline{A}_{02}$ is "circularly polarized" at the point $\underline{K}=0$. This gives us a hint as to the special advantages of the use of circular polarization components for equations of the above form. We introduce circular polarization components for on-axis quantities in the following manner: For the transmitting characteristic

$$
\underline{S}_{10}(0)=S_{+}^{0} \underline{e}_{+}+S_{-}^{O} \underline{e}
$$

and for the receiving characteristic

$$
\underline{A}_{02}(0)=A_{+}^{O} \underline{\bar{e}}_{+}+A_{-}^{O} \underline{\underline{e}}_{-},
$$

where the superposed bar denotes the complex conjugate (as usual) and

$$
\underline{\mathrm{e}}_{+} \equiv\left(\underline{\mathrm{e}}_{\mathrm{x}}+\mathrm{ie}_{\mathrm{y}}\right) / \sqrt{2}, \quad \underline{\mathrm{e}}_{-} \equiv\left(\underline{\mathrm{e}}_{\mathrm{x}}-\underline{\mathrm{i}}_{\mathrm{e}}\right) / \sqrt{2}
$$


(The ordered pair of vectors $\underline{\bar{e}}_{+}, \underline{\bar{e}}_{-}$in $(9 b)$ is reciprocal to the ordered pair $\underline{e}_{+}$, $\underline{e}_{-}$in $\left.(9 a).\right)$ The formulas for transformation to circular components are thus determined as

$$
\begin{array}{ll}
S_{x}^{0}=\left(S_{+}^{0}+S_{-}^{0}\right) / \sqrt{2}, & S_{y}^{0}=i\left(S_{+}^{0}-S_{-}^{0}\right) / \sqrt{2}, \\
A_{x}^{0}=\left(A_{+}^{0}+A_{-}^{0}\right) / \sqrt{2}, & A_{y}^{0}=-i\left(A_{+}^{0}-A_{-}^{0}\right) / \sqrt{2} .
\end{array}
$$

The scalar products in (8) become

$$
\begin{aligned}
& A_{+}^{O} S_{+}^{O}+A_{-}^{O} S_{-}^{O}=D_{A}(0) \\
& i A_{+}^{O} S_{+}^{O}-i A_{-}^{O} S_{-}^{O}=D_{B}(0)
\end{aligned}
$$

As is apparent, we have chosen the notation so that $\mathrm{A}_{+}$and $\mathrm{A}_{-}$ represent receptivities to the correspondingly labelled circular components of $\underline{S}_{10}$. The coupling is of course consistent with the polarization matching theorem (1.6-18). Equations (11) show clearly that if the receiving antenna were to respond to only one circular component of polarization, then that component, but not the other, could still be measured using one or the other of the two equations. If both equations were to be used, the compatibility condition (required by the vanishing of the determinant) should be satisfied within experimental error.

\subsection{Generalized Two-Identical-Antenna Techniques}

The technique to be described is formulated for non-reciprocal antennas, assuming that one possesses the adjoint of the antenna to be measured. If the antenna to be measured is reciprocal ( $\equiv$ selfadjoint), then the assumption is that one possesses duplicate 
antennas. $^{13}$ This is no doubt the more likely case but the more general formulation can be given with essentially no extra algebraic complication. Under the main assumption we are assured that the receiving characteristic of one antenna will be related to the transmitting characteristic of the other by reciprocity relations whether or not the antennas are individually reciprocal.

Granted the assumption of duplicate or mutually adjoint antemas, no additional assumptions are required to permit formulation and solution of equations for on-axis gain and polarization characteristics of both antennas. Additional a priori information, ordinarily qualitative, is required only for resolution of squareroot sign ambiguities.

Certain commonly occurring types of symmetry permit one additionally to obtain solutions for off-axis values of gain and polarization.

For definiteness we assume transmission from left to right (as usual); limit the discussion to the determination of the right-side characteristics of the two (in general distinct) antennas labelled $S$ and $S^{a}$, say; and choose to formulate equations for the direct determination of transmitting characteristics, leaving receiving characteristics to be determined by reciprocity. Under these ground rules, the remaining problem consists of at most two parts:

(a) Transmit from $\mathrm{S}$ to $\mathrm{S}^{\mathrm{a}}$; formulate equations for $\underline{\mathrm{S}}_{10}$, find $\underline{\mathrm{S}}_{\mathrm{O} 1}^{\mathrm{a}}$ by reciprocity;

13 The idea of using a reflecting surface or mirror to produce an image antenna is not fully applicable, even if the antenna is reciprocal: Coupling-product data can be obtained by extrapolation or by conventional techniques, but not by transverse scanning. The coupling-product equations can be formulated and solved for on-axis values provided on-axis polarization is known and not circular. It should be noted that the image antemn, being a mirror image, cannot in general be considered an identical antenna even with a perfectly reflecting surface of infinite area. 
(b) Transmit from $S^{a}$ to $S$; formulate equations for $\underline{S}_{10}^{a}$, find $\underline{S}_{01}$ by reciprocity.

If reciprocity (in the ordinary sense) applies, the superscript "a" is without effect and may be ommitted; the two cases reduce to one.

Inasmuch as the algebraic problem is in all cases substantially identical, it will be sufficient to consider only case (a) explicitly.

Both antennas are initially to be described in the same orientation and position relative to the fixed coordinate system Oxyz. When one of the antennas is placed and oriented to serve in reception, its description relative to fixed coordinates will be changed accordingly. Indeed, the phase factor $\exp (1 \gamma d)$ introduced by the axial translation can be regarded either as a modification of the receiving characteristic of the receiving antenna or as a property of the transmission path. We make the latter point of view explicit by referring the description of the receiving antenna to the shifted coordinate system $0^{\prime} x y z^{\prime}$, where $0^{\prime}$ is at point $(0,0, d)$ in the original system Oxyz (figure 7).

We shall need to consider the receiving antenna in two receiving orientations, differing by a 90 degree rotation around the z-axis. The operative characteristics of the receiving antenna in these two orientations will be distinguished by single and double primes.

Let the adjoint antenna be rotated into the first receiving orientation. This requires 180 degrees rotation around a transverse axis, say the $y$-axis. The operative receiving characteristic of the rotated antenna is then

$$
\underline{s}_{02}^{a^{\prime}}(\underline{K})=P_{y 2} \underline{S}_{01}^{a}(\underline{K})
$$


where $\mathrm{P}_{y_{2}}$ is a notation for the transformation (of $\underline{S}_{-1}^{\mathrm{a}}$ ) produced by the prescribed rotation. Now the transformed function $\mathrm{P}_{\mathrm{y} 2} \underline{\mathrm{S}}_{\mathrm{O}}^{\mathrm{a}}$ is related by reciprocity, $(1.5-4)$, to the similarly transformed function $\mathrm{P}_{y 2} \mathrm{~S}_{10}$. Thus, if the reciprocity relation is written in vector form, we have

$$
\eta_{0} \underline{S}_{02}^{a^{\prime}}(\underline{K})=\underline{\underline{n}} \cdot P_{y 2} \underline{S}_{10}(-\underline{K})
$$

and if it is written in $x, y$ component form we have

$$
n_{0}\left(\begin{array}{l}
s_{02 x}^{a^{\prime}}(\underline{K}) \\
S_{02 y}^{a^{\prime}}(\underline{K})
\end{array}\right)=\left(\begin{array}{cc}
n_{1} c^{2}+n_{2} s^{2} & \left(n_{1}-n_{2}\right) c s \\
\left(n_{1}-n_{2}\right) c s & n_{1} s^{2}+n_{2} c^{2}
\end{array}\right)\left(\begin{array}{c}
-s_{10 x}\left(k_{x},-k_{y}\right) \\
s_{10 y}\left(k_{x},-k_{y}\right)
\end{array}\right)
$$

Here the square matrix is determined by (4.1-4) and the column matrix on the right contains the $x, y$ components of $\mathrm{P}_{y} 2 \underline{S}_{10}(-\underline{K})$. We may now evaluate the coupling product $\underline{S}_{10}(\underline{K}) \cdot \underline{S}_{02}^{a}(\underline{K}) \equiv D^{\prime}(\underline{K})$. Some degree of abbreviation is indispensable; we use

$S_{x}=S_{10 x}(\underline{K}), S_{y}=S_{10 y}(\underline{K}), S_{x}^{V}=S_{10 x}\left(k_{x},-k_{y}\right), S_{y}^{V}=S_{10 y}\left(k_{x},-k_{y}\right)$.

and obtain

$$
\begin{aligned}
& -\left(n_{1} c^{2}+n_{2} s^{2}\right) S_{x} S_{x}^{V}+\left(n_{1}-n_{2}\right) \operatorname{scs} S_{x} S_{y}^{V}+ \\
& -\left(n_{1}-n_{2}\right) s_{y} S_{y} S_{x}^{V}+\left(n_{1} s^{2}+n_{2} c^{2}\right) S_{y} S_{y}^{v}=n_{0} D^{\prime}(\underline{K}) .
\end{aligned}
$$

This is the first of the desired "measurement equations" relating the mathematical expression of the coupling product to its empirically determined values. It is interesting that the expression is invariant with respect to the interchange of $k_{y}$ and $-k_{y}$; the empirical D' (K) should also have thịs symmetry. Further, since $D^{\prime}(\underline{K})$ is related to $b_{0}^{\prime}(\underline{P})$ by $(3.1-1)$, the $b_{0}^{\prime}(\underline{P})$ data should have 
the corresponding property of invariance with respect to the interchange of $y$ and $-y$. These general constraints should be experimentally useful.

To obtain the second measurement equation, we rotate the receiving antenna, as described by (1), 90 degrees around the $z$ axis in the direction $x$ to $y$ (cf. $(4.1-7))$. Using the notation $\mathrm{P}_{24}$ for this rotation and applying the reciprocity relation, as in (2), we obtain

$$
\eta_{0} \underline{S}_{02}^{a^{\prime \prime}}(\underline{K})=\underline{\underline{n}} \cdot P_{24} P_{y 2} \underline{S}_{10}(-\underline{K}) .
$$

In $x, y$ component form this is

$n_{0}\left(\begin{array}{l}s_{02 x}^{a^{\prime \prime}}(\underline{K}) \\ s_{02 y}^{a^{\prime \prime}}(\underline{K})\end{array}\right)=\left(\begin{array}{ll}n_{1} c^{2}+n_{2} s^{2} & \left(n_{1}-n_{2}\right) s c \\ \left(n_{1}-n_{2}\right) s c & n_{1} s^{2}+n_{2} c^{2}\end{array}\right)\left(\begin{array}{l}-s_{10 y}\left(k_{y}, k_{x}\right) \\ -s_{10 x}\left(k_{y}, k_{x}\right)\end{array}\right)$.

Using the abbreviations

$$
s_{x}^{d}=s_{10 x}\left(k_{y}, k_{x}\right), \quad s_{y}^{d}=s_{10 y}\left(k_{y}, k_{x}\right)
$$

as well as $S_{x}$ and $S_{y}$ in (4), we find for the coupling product $\underline{S}_{10}(\underline{K}) \cdot \underline{S}_{02}^{a \prime \prime}(K) \equiv D^{\prime \prime}(\underline{K})$ the expression

$$
\begin{aligned}
& -\left(n_{1} c^{2}+n_{2} s^{2}\right) s_{x} s_{y}^{d}-\left(n_{1}-n_{2}\right) s c s_{x} s_{x}^{d}+ \\
& -\left(n_{1}-n_{2}\right) s c s_{y} s_{y}^{d}-\left(n_{1} s^{2}+n_{2} c^{2}\right) s_{y} s_{x}^{d}=n_{0} D^{\prime \prime}(\underline{K}) .
\end{aligned}
$$

In this case the coupling product is invariant with respect to the interchange of $k_{x}$ and $k_{y}$, and again this constraint should be experimentally useful.

In the remainder of this subsection we discuss briefly some conditions and methods for determining components of $\underline{S}_{10}$ from (5) 
and (9), assuming that the D's are given for the value of $\underline{K}$ of interest. When evaluated for $\underline{\mathrm{K}} \neq 0$, the $\operatorname{six}$ quantities $\mathrm{S}_{x}, \mathrm{~S}_{\mathbf{y}}, \mathrm{S}_{\mathrm{x}}^{\mathrm{V}}$, $S_{y}^{V}, S_{x}^{d}$, and $S_{y}^{d}$ appearing in these two equations are in general distinct and unknown; obviously, some specialization or additional data are required. We consider two cases: evaluation on-axis, and a simple type of symmetry.

(i) Evaluation on axis - Evaluated on-axis, the six unknowns reduce to two

$$
S_{x}^{O}=S_{10 x}(0), \quad S_{y}^{0}=S_{10 y}(0),
$$

and coupling-product equations reduce to

$$
\begin{aligned}
-\left(S_{x}^{O}\right)^{2}+\left(S_{y}^{O}\right)^{2} & =D^{\prime}(0) \eta_{0} / Y_{O}, \\
-2 S_{x}^{O} S_{y}^{O} & =D^{\prime \prime}(0) \eta_{0} / Y_{0} .
\end{aligned}
$$

(The key to this reduction is the observation that $Y_{O}$ is the common value of $\eta_{1}$ and $\eta_{2}$ on-axis.) Sub-cases under this case occur if the polarization on-axis is considered known. One or the other of (11) will suffice, no matter what that polarization may be.

Suppose, for example, that $\rho=S_{y}^{0} / S_{x}^{0}$ is considered known and not equal to \pm 1 ; then from (11a) we may obtain

$$
S_{X}^{O}=\left(\frac{\eta_{0} D^{\prime}(0)}{Y_{0}\left(\rho^{2}-1\right)}\right)^{1 / 2}
$$

This, together with $S_{y}^{O}=\rho S_{x}^{O}$, gives us the on-axis pattern vector in terms of $D^{\prime}(0)$ (up to a sign). From the expression (1.6-6) for power gain we find

$$
G_{1}(0)=\frac{4 \pi k^{2}\left(|\rho|^{2}+1\right)\left|D^{\prime}(0)\right|}{\left(1-\left|S_{00}\right|^{2}\right)\left|\rho^{2}-1\right|} .
$$


We can obtain an interesting form for this result by expressing $D^{\prime}(0)$ in terms of the integral of $b_{0}^{\prime}(\underline{P})$, as in the deconvolution relation, (3.1-3). Thus,

$$
G_{1}(0)=\frac{k^{2}}{\pi} \frac{\left|1-\Gamma_{L}^{\prime} S_{O O}^{\prime}\right|}{1-\left|S_{O O}\right|^{2}} \frac{|\rho|^{2}+1}{\left|\rho^{2}-1\right|} \frac{1}{\left|a_{O}\right|}\left|\int b_{O}^{\prime}(\underline{P}) d \underline{P}\right| .
$$

(This result, as well as the version of it with $\rho=0$, has been presented previously $[41,44]$.) The essential simplicity of the result is somewhat obscured by the presence of the mismatch factors. If we assume a polarization match $(\rho=0, \pm i$, or $\infty)$, a conjugate impedance match $\left(\Gamma_{L}^{\prime}=\bar{S}_{O O}^{\prime}\right)$, and $S_{O O}=S_{O O}^{\prime}$ (as appropriate for mutually adjoint or for identical antennas), we have

$$
G_{1}(0)=\frac{k^{2}}{\pi} \cdot \frac{1}{\left|a_{0}\right|}\left|\int b_{0}^{\prime}(\underline{P}) d \underline{p}\right| .
$$

For the effective area, using (1.6-21b), we obtain the remarkably simple expression

$$
\sigma_{1}^{\mathrm{a}}(0)=\frac{1}{\left|\mathrm{a}_{0}\right|}\left|\int \mathrm{b}_{\mathrm{o}}^{\prime}(\underline{\mathrm{p}}) \mathrm{d} \underline{\mathrm{p}}\right|
$$

Equation (13) has been successfully applied experimentally [8], and (15) has been tested analytically in a special case (see example III-2.2-12). In the analytical test the two identical antennas were taken to be $x$-oriented elementary electric-dipole antennas, assumed lossless and reciprocal. In this case one does indeed obtain the expected result $\sigma_{1}(0)=3 \lambda^{2} /(8 \pi)$.

More generally, the polarization is not known and it is necessary to solve (11) as simultaneous quadratics. A solution in terms of circular polarization components is convenient and useful. Using the definitions in (4.1-9a), one obtains 


$$
\begin{aligned}
& \left(S_{+}^{O}\right)^{2}=-\frac{\eta_{O}}{2 Y_{O}}\left[D^{\prime}(0)-i D^{\prime \prime}(0)\right], \\
& \left(S_{-}^{O}\right)^{2}=-\frac{\eta_{O}}{2 Y_{O}}\left[D^{\prime}(0)+i D^{\prime \prime}(0)\right] .
\end{aligned}
$$

From these equations we obtain four pairs of values for the $x$, y components:

$$
\begin{aligned}
& S_{x}^{0}= \pm \frac{i}{2} \sqrt{\frac{\eta_{O}}{Y_{0}}}\left[\sqrt{D^{\prime}(0)+i D^{\prime \prime}(0)} \pm \sqrt{1)^{\prime}(0)-i D^{\prime \prime}(0)}\right] \\
& S_{y}^{0}= \pm \frac{1}{2} \sqrt{\frac{\eta_{0}}{Y_{0}}}\left[\sqrt{D^{\prime}(0)+i D^{\prime \prime}(0)}+\sqrt{D^{\prime}(0)-i D^{\prime \prime}(0)}\right] .
\end{aligned}
$$

(The double signs are correlated vertically but not horizontally.) Which solution-pair pertains to a given measurement cannot be determined from the equations alone. It would seem that ordinarily the overall plus-minus sjgns should be of no significance. The remaining sign-chojce does affect the determination of the polarization index $\rho=S_{y}^{o} / S_{x}^{o}$. If we write $\rho^{(u)}$ and $\rho^{(l)}$ for the values associated with the upper and the lower signs, respectively, we find

$$
\rho^{(u)}(l)=-1 \text {. }
$$

With the aid of this equation a modicum of a priori information about the magnitude and/or the phase of $\rho$ should ordinarily be sufficient to resolve the ambiguity. For example, if the antenna is known to be approximately linearly polarized (on-axis) in a certain direction (we may choose the $y$-axis in that direction), then $|\rho|$ is distinctly greater than unity and one would choose $\rho^{(u)}$ or $\rho^{(l)}$ accordingly. However, if the polarization is nearly circular, the difference between the two indices becomes relatively small and the choice could be difficult. 
The squared magnitude $\left|S_{x}^{O}\right|^{2}+\left|S_{y}^{0}\right|^{2}$, which determines the onaxis power gain, is unambiguous. In fact, for this gain we find

$$
G_{1}(0)=\frac{2 \pi k^{2}\left[\left|D^{\prime}(0)+i D^{\prime \prime}(0)\right|+\left|D^{\prime}(0)-i D^{\prime \prime}(0)\right|\right]}{1-\left|S_{0 O}\right|^{2}} .
$$

(ii) Additional solutions permitted by symmetry. Certain types of symmetry enable one to determine certain off-axis values of the unknown functions. We consider one type of symmetry of frequent occurrence, exemplified by the fields of (a) rectangular waveguide open-ended or with a pyramidal horn, fed by the $\mathrm{TE}_{10}$ mode in the waveguide; (b) circular waveguide open-ended or with a circularly symetric horn, fed by the $\mathrm{TE}_{11}$ mode in the waveguide; and (c) a transverse electric dipole. In these examples the symmetry may be analytically specified in terms of spectral components by

$$
\begin{aligned}
& S_{10 x}\left(-k_{x}, k_{y}\right)=S_{10 x}\left(k_{x},-k_{y}\right)=-S_{10 x}\left(k_{x}, k_{y}\right) \\
& S_{10 y}\left(-k_{x}, k_{y}\right)=S_{10 y}\left(k_{x},-k_{y}\right)=S_{10 y}\left(k_{x}, k_{y}\right)
\end{aligned}
$$

(Here a specific orientation of structures and fields has of course been assumed.) We note in particular that $\mathrm{S}_{10 x}$ must vanish on the coordinate axes. Hence, the four unknown functions involved in (5) are reduced to the single one, $S_{10 y}\left(k_{x}, 0\right)$ on the line $k_{y}=0$, and to the single function $\mathrm{S}_{10 y}\left(0, \mathrm{k}_{\mathrm{y}}\right)$ on the 1 ine $\mathrm{k}_{\mathrm{x}}=0$. From (5) we immediately obtain the separate equations determining these two functions,

$$
\begin{aligned}
& n_{2}\left[s_{10 y}\left(k_{x}, 0\right)\right]^{2}=n_{0} D^{\prime}\left(k_{x}, 0\right), \\
& n_{1}\left[s_{10 y}\left(0, k_{y}\right)\right]^{2}=n_{0} D^{\prime}\left(0, k_{y}\right) .
\end{aligned}
$$


Next we notice that on the diagonal line $k_{y}=k_{x}$, we have $S_{x}^{d} \equiv S_{x}$ and $S_{y}^{d} \equiv S_{y}$. Additionally, the symmetry furnishes $S_{x}=-S_{x}^{v}$ and $S_{y}=S_{y}^{v}$. Hence, of the six unknown functions involved in (5) and (9) essentially only two distinct ones survive,

$$
u_{x} \equiv s_{10 x}\left(k_{x}, k_{x}\right), \quad u_{y} \equiv S_{10 y}\left(k_{x}, k_{x}\right)
$$

say. Further, the two quadratics can easily be solved for these unknowns, as the following intermediate results show:

$$
\begin{aligned}
& \left(u_{x}-u_{y}\right)^{2} n_{2}=n_{0}\left[D^{\prime}\left(k_{x}, k_{x}\right)+D^{\prime \prime}\left(k_{x}, k_{x}\right)\right], \\
& \left(u_{x}+u_{y}\right)^{2} n_{1}=n_{0}\left[D^{\prime}\left(k_{x}, k_{x}\right)-D^{\prime \prime}\left(k_{x}, k_{x}\right)\right] .
\end{aligned}
$$

Hence in this case we can obtain solutions on the four lines, $k_{x}=0, k_{y}=0$, and $k_{y}= \pm k_{x}$.

Equations (5) and (9) may of course be expressed in radial $\left(\underline{k}_{1}\right)$ and tangential $\left(\underline{\kappa}_{2}\right)$ components. The resulting forms are simpler in appearance and may in some cases (e.g., consideration of circular symetry) be more convenient than the forms (5) and (9). Consequently we conclude this subsection by recording the following alternative forms for (5) and (9).

$-n_{1} S_{10}\left(1 ; k_{x},-k_{y}\right) S_{10}(1, \underline{K})+n_{2} S_{10}\left(2 ; k_{x},-k_{y}\right) S_{10}(2, \underline{K})=n_{0} D^{\prime}(\underline{K})$,

$-n_{1} S_{10}\left(1 ; k_{y}, k_{x}\right) S_{10}(1, \underline{K})+n_{2} S_{10}\left(2 ; k_{y}, k_{x}\right) S_{10}(2, \underline{K})=\eta_{0} D^{\prime \prime}(\underline{K})$.

\subsection{Generalized Three-Antenna Techniques}

In this subsection we discuss the analysis involved in techniques for determination of both power gain and polarization using three unknown (dissimilar) antennas. We require an antenna $T$, to 
Sec. 4.3]

be used only in transmitting; an antenna $R$, to be used only in reception; and an antenna $S$ to be used in both receiving and transmitting modes. We do not need to inquire whether either of the antennas $T$ and $R$ is reciprocal or even capable of operating in a "reversed" mode. We do require either that antenna $S$ be reciprocal or, if not reciprocal, capable of being "switched" to become its own adjoint $S^{a}$.

It is interesting and important that some kind of reciprocity ${ }^{14}$ is indispensably required a priori information. This requirement cannot be avoided by increasing the number of antennas involved-even to the extent of using all possible combinations of $n$ transmitting and $m$ receiving antennas. But when the reciprocity requirement is met, as with a reciprocal or switchable antenna $S, 3$ antennas are sufficient. (Note that in the switchable case, $S$ and its adjoint do not coexist. Actual simultaneous possession of both $S$ and $S^{a}$ would distinctly change the character of the measurement problem: in this case one could use the generalized 2-identical-antenna technique of the preceding subsection.)

of course the use of 3 antennas in a measurement scheme is not in itself new. The use of 3 antennas in the roles of $T, R$, and $S$ (S being reciprocal) is recognizable in a discussion in Vol. 12 of the MIT Radiation Laboratory Series-material originating in the early 1940's [45, p. $582 \mathrm{ff}]$. However, in that discussion simplifying assumptions regarding polarization were made and the analytical problem was reduced to the use of scalar equations ${ }_{14}$ Conceivably some linear relation, other than that provided by ordinary or
adjoint reciprocity, between receiving and transmitting characteristics
might be known a priori. This would not in general lead to the relatively
simple equations that are obtained under the assumption made. 
involving gain only. Analogous schemes using three electroacoustic transducers coupled by a flujd medium are well known [46]. The application of the requisite electroacoustic reciprocity relations was introduced by Maclean [47] and by Cook [48] in 1940 and 1941 , respectively.

In this discussion we consider only on-axis values of antenna characteristics and refer to these quantities as the antenna characteristics. Determination of off-axis characteristics by threeantenna techniques has not yet been seriously considered, to our knowledge. (The corresponding electroacoustics problem, which is much simpler, has recently been completely solved under minimum assumptions in work as yet unpublished.)

When antenna polarization characteristics are fully taken into account, as in the present discussion, three-antenna techniques for the determination of on-axis characteristics require less a priori information than any other technique. Thus versions of the threeantenna technique have been the method of choice in several critical applications $[\mathrm{e} . \mathrm{g} ., 9,11]$.

The problem now posed is taken to be the determination of the receiving characteristic $\underline{R}_{02}(0)$ of the antenna $R$, the transmitting characteristjc $\mathrm{T}_{-10}(0)$ of the antenna $\mathrm{T}$, and the four "right-side" characteristics $\underline{S}_{10}(0), \underline{S}_{01}(0), \underline{S}_{10}^{a}(0), \underline{S}_{01}^{a}(0)$ of the antennas $S$ and $S^{a}$. We further choose to eliminate the receiving characteristics of $S$ and $S^{a}$ by means of the reciprocity relations, which for $\underline{K}=0$ are simply

$$
\eta_{0}-_{01}(0)=Y_{0} \underline{S}_{10}^{\mathrm{a}}(0), \quad \eta_{0} \underline{S}_{01}^{\mathrm{a}}(0)=Y_{0} \underline{S}_{10}(0) .
$$


In this discussion we use the notation

$$
R_{x}=R_{02 x}(0), S_{x}=S_{10 x}(0), S_{x}^{a}=S_{10 x}^{a}(0), T_{x}=T_{10 x}(0) \quad(x=x, y)
$$

for the components of the four remaining vector unknowns. Certainly, to determine these 8 (complex) quantities we need a system of 8 (complex) equations; the "core" of the problem, however, turns out to be the solution of six simultaneous quadratic equations for six of the unknowns. (This is the complete solution if antenna $S$ is reciprocal.) The remainder of the problem requires only the solution of two linear equations in two unknowns; this part will be called the "supplementary" problem.

The complete problem requires coupling-product data derived from transmission between antennas paired as shown in table 2 . Transmission is from left to right (as already implied in the notation), and for each antenna-pair considered, the receiving antenna is used in two orientations differing by 90 degree rotation around the z-axis. The first two columns in the table indicate two ways of utilizing the same data and are related by the interchange of the roles of $S$ and $S^{a}$. If the antenna $S$ is reciprocal $\left(S=S^{a}\right)$, the first two columns coincide and reduce to the third; only the core problem remains. It will be sufficient to formulate and solve the algebraic problem represented in the first column of the table; the corresponding formulations and solutions may be obtained for the second column by interchanging $S$ and $S^{a}$ and for the case of reciprocal S by eliminating the superscript "a" (and with it the equations thus rendered superfluous). 
Table 2. Antenna pairings.

\begin{tabular}{c|c|c|c}
\cline { 2 - 3 } & \multicolumn{2}{|c|}{$S \neq S^{\mathrm{a}}$} & $\mathrm{S}=\mathrm{S}^{\mathrm{a}}$ \\
\hline For "core" problem: & $\mathrm{T} \rightarrow \mathrm{R}$ & $\mathrm{T} \rightarrow \mathrm{R}$ & $\mathrm{T} \rightarrow \mathrm{R}$ \\
& $\mathrm{S} \rightarrow \mathrm{R}$ & $\mathrm{S}^{\mathrm{a}} \rightarrow \mathrm{R}$ & $\mathrm{S} \rightarrow \mathrm{R}$ \\
$\mathrm{T} \rightarrow \mathrm{S}^{\mathrm{a}}$ & $\mathrm{T} \rightarrow \mathrm{S}$ & $\mathrm{T} \rightarrow \mathrm{S}$ \\
\hline For "supplementary" & $\mathrm{S}^{\mathrm{a}} \rightarrow \mathrm{R}$ & $\mathrm{S} \rightarrow \mathrm{R}$ & $\cdots \cdots$ \\
\hline
\end{tabular}

For the transmission from $\mathrm{T}$ to $\mathrm{R}$, we have

$$
\mathrm{T}_{x} \mathrm{R}_{x}+\mathrm{T}_{y} \mathrm{R}_{y}=\mathrm{D}_{\mathrm{RT}}^{\prime}, \quad-\mathrm{T}_{x} \mathrm{R}_{y}+\mathrm{T}_{y} \mathrm{R}_{x}=\mathrm{D}_{\mathrm{RT}}^{\prime \prime} \cdot
$$

These equations are an instance of (4.1-8) (except that here chacteristics of both antennas are unknown). For the transmission from $S$ to $R$, we have

$$
S_{x} R_{x}+S_{y} R_{y}=D_{R S}^{\prime}, \quad-S_{x} R_{y}+S_{y} R_{x}=D_{R S}^{\prime \prime} \cdot
$$

These equations are similarly an instance of (4.1-8). For the transmission from $\mathrm{T}$ to $\mathrm{S}^{\mathrm{a}}$, we have

$$
-\mathrm{T}_{x} \mathrm{~S}_{x}+\mathrm{T}_{y} \mathrm{~S}_{y}=n_{0} \mathrm{D}_{\mathrm{ST}}^{1} / \mathrm{Y}_{0}, \quad-\mathrm{T}_{x} \mathrm{~S}_{y}-\mathrm{T}_{y} \mathrm{~S}_{\mathrm{x}}=\eta_{\mathrm{O}} \mathrm{D}_{\mathrm{ST}}^{\prime \prime} / \mathrm{Y}_{0} \cdot
$$

The receiving antenna, $\mathrm{S}^{\mathrm{a}}$, is here treated in the same way as was the receiving antenna in the two-antenna technique described in the preceding subsection (cf. (4.2-10)). This completes the formulation of the "core" problem. The solution is conveniently accomplished with the aid of circular polarization components, which are defined as in (4.1-9). Equations (3), (4), and (5) transform pair by pair to 


$$
\left.\left.\begin{array}{c}
T_{-} R_{-}=\frac{1}{2}\left(D_{R T}^{\prime}+i D_{R T}^{\prime \prime}\right) \equiv \Sigma_{R T}, \\
T_{+} R_{+}=\frac{1}{2}\left(D_{R T}^{\prime}-i D_{R T}^{\prime \prime}\right) \equiv \Delta_{R T} ;
\end{array}\right\} \begin{array}{c}
S_{-} R_{-}=\frac{1}{2}\left(D_{R S}^{\prime}+i D_{R S}^{\prime \prime}\right) \equiv \Sigma_{R S}, \\
S_{+} R_{+}=\frac{1}{2}\left(D_{R S}^{\prime}-i D_{R S}^{\prime \prime}\right) \equiv \Delta_{R S} ;
\end{array}\right\}
$$

For the squares of the individual circular polarization components one finds without difficulty (but note that neither this set of equations nor the preceding sets can be generated by straightforward advancement of subscripts)

$$
\begin{aligned}
& \left.\begin{array}{ll}
\mathrm{R}_{-}^{2}=-\frac{\mathrm{Y}_{\mathrm{O}}}{\eta_{\mathrm{O}}} \frac{\sum_{\mathrm{RT}} \sum_{\mathrm{RS}}}{\sum_{\mathrm{ST}}}, & \left(\mathrm{T}_{-} \mathrm{S}_{-} \neq 0\right) \\
\mathrm{R}_{+}^{2}=-\frac{\mathrm{Y}_{\mathrm{O}}}{n_{\mathrm{O}}} \frac{\Delta_{\mathrm{RT}} \Delta_{\mathrm{RS}}}{\Delta_{\mathrm{ST}}} & \left(\mathrm{T}_{+} \mathrm{S}_{+} \neq 0\right)
\end{array}\right\} \\
& \left.\begin{array}{ll}
S_{+}^{2}=-\frac{\eta_{0}}{Y_{0}} \frac{\Delta_{R S} \Delta_{S T}}{\Delta_{R T}}, & \left(T_{+} R_{+} \neq 0\right) \\
S_{-}^{2}=-\frac{\eta_{0}}{Y_{0}} \frac{\sum_{R S} \sum_{S T}}{\sum_{R T}} ; & \left(T_{-} R_{-} \neq 0\right)
\end{array}\right\} \\
& \left.\begin{array}{ll}
\mathrm{T}_{+}^{2}=-\frac{n_{\mathrm{O}}}{\mathrm{Y}_{\mathrm{O}}} \frac{\Delta_{\mathrm{RT}} \Delta_{\mathrm{ST}}}{\Delta_{\mathrm{RS}}}, & \left(\mathrm{S}_{+} \mathrm{R}_{+} \neq 0\right) \\
\mathrm{T}_{-}^{2}=-\frac{\eta_{\mathrm{O}}}{\mathrm{Y}_{\mathrm{O}}} \frac{\sum_{\mathrm{RT}}\left[\Gamma_{\mathrm{ST}}\right.}{\sum_{\mathrm{RS}}} . & \left(\mathrm{S}_{-} \mathrm{R}_{-} \neq 0\right)
\end{array}\right\}
\end{aligned}
$$


The inequalities shown in parentheses (which in effect prohibit circular polarizations) must be in force if indeterminacy in the respective associated equations is to be avoided. If circular polarizations do occur, then obviously many special cases are possible. For example, if the transmitting characteristic of antemna $S$ is (very nearly) right circularly polarized $\left(S_{y}=\right.$ is $x$ ), then (very nearly) $S_{-}=0, \sum_{R S}=0, \sum_{S T}=0$, and $R_{-}$and $T_{-}$become experimentally indeterminate. However, the determination of the other circular components of $\mathrm{R}$ and $\mathrm{T}$, and the determination of the transmitting characteristics of antenna $S$ itself, would present no special difficulty. In practice, if a partial solution such as this is not sufficient, one might include additional antennas in the scheme. Antennas capable of being switched between right and left circular polarizations have been used for similar reasons, usually in less complicated circumstances.

It remains to dispose of what we called the supplementary problem earlier in this discussion. If antenna $S$ is reciprocal, there is no supplementary problem; if $S$ is not reciprocal, it remains to determine $\underline{S}_{10}^{\mathrm{a}}(0)$. As suggested in the first column of table 2, this can be done by measurement with the receiving antenna $\mathrm{R}$, for which calibration is presumptively available as a result of the core problem. Equations (4.1-11) are applicable; in notation adapted to the present context those equations become

$$
\begin{gathered}
R_{+} S_{+}^{a}+R_{-} S_{-}^{a}=D_{R S}^{\prime}{ }^{a} \\
i R_{+} S_{+}^{a}-i R_{-} S_{-}^{a}=D_{R S^{\prime \prime}}^{a} .
\end{gathered}
$$

Here complete solvability requires $R_{+} R_{-} \neq 0$. 
Effective areas and power gains for antennas $R, S, S^{a}$, and $T$ (as pertinent) are readily expressed in terms of circular polarization components, which by reference to (4.1-9), are seen to be normalized so that

$$
\left|A_{x}\right|^{2}+\left|A_{y}\right|^{2}=\left|A_{+}\right|^{2}+\left|A_{-}\right|^{2}
$$

Thus, for example, we obtain for antenna $S$ from (10) and (1.6-6)

$$
\left.\mathrm{G}_{1}(0)=\frac{4 \pi \mathrm{k}^{2}}{1-\left|\mathrm{S}_{\mathrm{OO}}\right|^{2}}\left(\left|\frac{\Delta_{\mathrm{RS}} \Delta_{\mathrm{ST}}}{\Delta_{\mathrm{RT}}}\right|\right)+\left|\frac{\sum_{\mathrm{RS}} \sum_{\mathrm{ST}}}{\sum_{\mathrm{RT}}}\right|\right) \text {. }
$$

The corresponding effective area is given by $(1.6-21 a)$ or $(1.6-21 b)$, as appropriate.

Algebraic values for the individual linear polarization components may be obtained from (9), (10), or (11), as desired. For example, for antenna $S$

$$
\begin{aligned}
& S_{x}= \pm i \sqrt{\frac{\eta_{0}}{Y_{0}}}\left(\sqrt{\frac{\sum_{R S} \sum_{S T}}{\sum_{R T}}} \pm \sqrt{\frac{\Delta_{R S} \Delta_{S T}}{\Delta_{R T}}}\right), \\
& S_{y}= \pm \sqrt{\frac{n_{0}}{Y_{0}}}\left(\sqrt{\frac{\sum_{R S} S_{S T}}{\sum_{R T}}} \mp \sqrt{\frac{\Delta_{R S} \Delta_{S T}}{\Delta_{R T}}}\right),
\end{aligned}
$$

where the double signs are correlated vertically but not horizontally. As far as sign ambiguities are concerned, these equations have the same algebraic structure as (4.2-17) and the earlier discussion is again applicable. 


\section{APPENDICES TO CHAPTER I I}

\subsection{Appendix A: Reciprocity Theorems in the PWSM Context}

The domain of the electromagnetic fields under consideration is the source-free region $V$ bounded externally by the surfaces $F_{1}$ and $\mathrm{F}_{2}$ and internally by the closed surface $\mathrm{S}_{0}+\mathrm{S}_{\mathrm{a}}$, which encloses the source or detector associated with the antenna considered. (See subsection 1.1 and figure 1.) A passive antenna is a scatterer (sometimes called a "loaded scatterer"); if the structure is merely a scatterer, the surface $S_{0}+S_{a}$ is irrelevant and may be disregarded.

We shall write Maxwell's equations in a form especially suited to the purposes of the present discussion. We associate with the given system, described by the constitutive equations (1.5-1), the "Maxwellian" operator

$$
M=\left(\begin{array}{cc}
i \omega \underline{\underline{\varepsilon}}^{\cdot} & i \omega \underline{\underline{\tau}} \cdot+\nabla x \\
-i \omega \underline{\underline{\underline{v}}} \cdot+\nabla x & -i \omega \underline{\underline{\mu}} \cdot
\end{array}\right\}
$$

and place the vectors $\underline{E}$ and $\underline{H}$ in the column matrix

$$
\Phi=\left(\begin{array}{l}
\underline{E} \\
\underline{H}
\end{array}\right),
$$

so that Maxwell's equations for a possible field in the system are expressed by

$$
\mathrm{M} \Phi=0 .
$$

Using the constitutive equations (1.5-2) we similarly obtain for the Maxwellian operator associated with the adjoint system

$$
\tilde{M}=\left(\begin{array}{cc}
i \omega \underline{\underline{\tilde{\varepsilon}}} \cdot & -i \omega \underline{\underline{\tilde{v}}} \cdot+\nabla \times \\
i \omega \underline{\underline{\tilde{\tau}}} \cdot+\nabla \times & -i \omega \underline{\underline{\underline{\mu}}} \cdot
\end{array}\right),
$$


where $\tilde{M}$ is in a natural sense the transpose of the operator $M$. Maxwell's equations for the fields in the adjoint system take the form

$$
\tilde{M} \Phi=0 \text {. }
$$

The adjoint system represents a possible physical embodiment of the mathematical concept of adjoint differential expressions, which may be defined as follows. Let

$$
\Phi^{\prime}=\left(\begin{array}{l}
\underline{F}^{\prime} \\
\underline{G}^{\prime}
\end{array}\right), \quad \Phi^{\prime \prime}=\left(\begin{array}{l}
\underline{F}^{\prime \prime} \\
\underline{G}^{\prime \prime}
\end{array}\right),
$$

where $\underline{F}^{\prime}, \underline{G}^{\prime}, \underline{F}^{\prime \prime}$, and $\underline{G}^{\prime \prime}$ are arbitrary differentiable vector functions defined in $V$. Then we can associate a unique adjoint differential expression $M^{\mathrm{a}}$ with $M$ by requiring that

$$
\tilde{\Phi}^{\prime \prime} \cdot M \Phi^{\prime}-\tilde{\Phi}^{\prime} \cdot M^{\mathrm{a}} \Phi^{\prime \prime} \equiv D,
$$

where D must be a divergence expression, be an identity in $\Phi^{\prime}$ and $\Phi^{\prime \prime}$. This leads to

$$
M^{\mathrm{a}}=\tilde{M}
$$

and

$$
D=\nabla \cdot\left(\underline{F}^{\prime} \times \underline{G}^{\prime \prime}-\underline{F}^{\prime \prime} \times \underline{G}^{\prime}\right) .
$$

Thus the adjoint operator turns out to be the transpose. Al

$\overline{\mathrm{Al}}$ To avoid possible confusion, we emphasize that the adjoint arising here is definable essentially by transposition (without complex conjugation); it is not the Hermitian adjoint that is frequently useful in other physical problems. For discussion of the theory of adjoint differential expressions see especially Lanczos [49] or (for ordinary differential equations) CourantHilbert [50]. Equation (5a) incidentally contains the nontrivial result that the operator $\nabla \times$ is self-adjoint. 
We may now easily obtain the basic theorem for our purposes. If we replace $\underline{F}^{\prime}, \underline{G}^{\prime}$ and $\underline{F}^{\prime \prime}, \underline{G}^{\prime \prime}$ by $\underline{E}^{\prime}, \underline{H}^{\prime}$ and $\underline{E}^{\prime \prime}, \underline{H}^{\prime \prime}$, which satisfy (1) and (2), respectively, we have

$$
\nabla \cdot\left(\underline{E}^{\prime} \times \underline{H}^{\prime \prime}-\underline{E}^{\prime \prime} \times \underline{H}^{\prime}\right)=0
$$

throughout the region $V$. This is a generalization of the wellknown Lorentz relation. It may very easily be modified to include the effects of both electric and magnetic current sources embedded in $V$ [37]. Here, however, we are interested in excitation of the systems by means of jncident waves (in space or waveguide). With or without current-source terms, (6) characterizes what may properly be called adjoint reciprocity.

If the constitutive tensors obey the symmetry relations

$$
\underline{\underline{\varepsilon}}=\underline{\underline{\tilde{\varepsilon}}}, \underline{\underline{\mu}}=\underline{\underline{\tilde{\mu}}} \text {, and } \underline{\underline{\underline{\tau}}}=-\underline{\underline{\tilde{v}}}
$$

then (as may be seen from (1.5-1, -2)) the adjoint system and the original system are identical (and $\mathrm{M}^{\mathrm{a}}=\tilde{\mathrm{M}}=\mathrm{M}$ ). In this case we say that the linear differential operators and the systems are self-adjoint--and ordinary reciprocity obtains. Equation 6 still holds; $\underline{E}^{\prime}, \underline{H}^{\prime}$ and $\underline{E}^{\prime \prime}, \underline{H}^{\prime \prime}$ may be interpreted as distinct electromagnetic fields in one and the same system.

To apply the generalized Lorentz relation, we first take the volume integral of the expression over the region $V$ and use the divergence theorem. This yields

$$
\int_{S_{0}} \underline{L} \cdot \underline{n}_{0} d S=\sum_{q} \int_{F_{q}} \underline{L} \cdot \underline{n}_{q} d \underline{R}
$$


where $\underline{L}=\underline{E}^{\prime} \times \underline{H}^{\prime \prime}-\underline{E}^{\prime \prime} \times \underline{H}^{\prime}$ and the unit normals are inward on $\mathrm{S}_{\mathrm{O}}$ and outward on $\mathrm{F}_{q}$ (as prescribed in subsections 1.1 and 1.2). Next we substitute the modal representations of the fields $\underline{E}^{\prime}, \underline{H}^{\prime}$ and $\underline{E}^{\prime \prime}, \underline{H}^{\prime \prime}$ on $\mathrm{S}_{\mathrm{O}}, \mathrm{F}_{1}$, and $\mathrm{F}_{2}$, using single and double primes to distinguish the spectral variables associated with the respective fields. One obtains after some analysis the generalized reciprocity lemma

$$
\begin{aligned}
& n_{0}\left(a_{0}^{\prime \prime} b_{0}^{\prime}-a_{0}^{\prime} b_{0}^{\prime \prime}\right)= \\
& \sum_{p} \int_{L} \sum_{n}\left[a_{p}^{\prime \prime}(n,-\underline{L}) b_{p}^{\prime}(n, \underline{L})-a_{p}^{\prime}(n, \underline{L}) b_{p}^{\prime \prime}(n,-\underline{L})\right] n_{n}(L) d \underline{L} .
\end{aligned}
$$

From this lemma, by making suitable special choices of excitation, we shall obtain the desired set of relations between the elements of the scattering matrix of the original system and the corresponding elements of the scattering matrix of the adjoint system.

For immediate reference we write down the scattering equations for the original system

$$
\begin{aligned}
& b_{0}^{\prime}=S_{o o} a_{0}^{\prime}+\sum_{p} \int_{\underline{L}} \sum_{n} S_{o p}(n, \underline{L}) a_{p}^{\prime}(n, \underline{L}) d \underline{L}, \\
& b_{q}^{\prime}(m, \underline{K})=S_{q o}\left(m, \underline{K} a_{o}^{\prime}+\sum_{p} \int_{L} \sum_{n} S_{q p}(m, \underline{K} ; n, \underline{L}) a_{p}^{\prime}(n, \underline{L}) d \underline{L} .\right.
\end{aligned}
$$

and those for the adjoint system

$$
\begin{aligned}
b_{0}^{\prime \prime} & =S_{o 0}^{a} a_{0}^{\prime \prime}+\sum_{p} \int_{\underline{L}} \sum_{n} S_{o p}^{a}(n, \underline{L}) a_{p}^{\prime \prime}(n, \underline{L}) d \underline{L}, \\
b_{q}^{\prime \prime}(m, \underline{K}) & =S_{q 0}^{a}(m, \underline{K}) a_{o}^{\prime \prime}+\sum_{p} \int_{L} \sum_{n} S_{q p}^{a}(m, \underline{K} ; n, \underline{L}) a_{p}^{\prime \prime}(n, \underline{L}) d \underline{L} .
\end{aligned}
$$

The superscript "a" distinguishes quantities characteristic of the adjoint system. The above sets of equations are instances of 
(1.3-2); each set is defined relative to the same basis field and reference surfaces.

To find the relationships between transmitting and receiving characteristics, we let $\underline{E}^{\prime}, \underline{H}^{\prime}$ and $\underline{E}^{\prime \prime}, \underline{H}^{\prime \prime}$ be the fields corresponding to excitation of the respective systems by the incident waves represented by the following set of spectral variables:

$$
\begin{array}{ll}
a_{0}^{\prime}=1, & a_{p}^{\prime}(n, \underline{L}) \equiv 0, \\
a_{0}^{\prime \prime}=0, & a_{p}^{\prime \prime}(n, \underline{L})=\delta_{p q} \delta_{n m} \delta(\underline{L}-\underline{K}) .
\end{array}
$$

From (9) we find

$$
-b_{0}^{\prime \prime} n_{0}=b_{q}^{\prime}(m,-\underline{K}) n_{m}(K)
$$

and from (10) and (11)

$$
b_{q}^{\prime}(m, \underline{K})=S_{q 0}(m, \underline{K}), \quad b_{0}^{\prime \prime}=S_{o q}^{a}(m, \underline{K}) .
$$

Hence [observing that $\eta_{m}(-K)=\eta_{m}(K)$ ] we obtain

$$
n_{\mathrm{o}} \mathrm{s}_{\mathrm{oq}}^{\mathrm{a}}(\mathrm{m}, \underline{\mathrm{K}})=-n_{\mathrm{m}}(\mathrm{K}) \mathrm{s}_{\mathrm{qo}}(\mathrm{m},-\underline{\mathrm{K}}) .
$$

Similarly, by interchanging the patterns of excitation we obtain

$$
n_{\mathrm{o}} \mathrm{s}_{\mathrm{oq}}(\mathrm{m}, \underline{\mathrm{K}})=-\eta_{\mathrm{m}}(\mathrm{K}) \mathrm{s}_{\mathrm{qo}}^{\mathrm{a}}(\mathrm{m}, \underline{-}-\underline{\mathrm{K}}) .
$$

To obtain the scattering reciprocity relations, we consider each system to be excited by an incident plane wave, as represented in the following scheme.

$$
\begin{aligned}
& a_{0}^{\prime}=0, \quad a_{p}^{\prime}(n, \underline{L})=\delta_{p p} \delta_{n n^{\prime}} \delta\left(\underline{L}-\underline{L}^{\prime}\right) ; \\
& a_{0}^{\prime \prime}=0, \quad a_{p}^{\prime \prime}(n, \underline{L})=\delta_{p p}{ }^{\prime \prime} \delta_{n n^{\prime \prime}} \delta\left(\underline{L}-\underline{L}^{\prime \prime}\right) .
\end{aligned}
$$


Fron (9) we find

$$
b_{p^{\prime \prime}}^{\prime}\left(n^{\prime \prime},-\underline{L}^{\prime \prime}\right) \eta_{n^{\prime \prime}}\left(L^{\prime \prime}\right)=b_{p}^{\prime \prime} ;\left(n^{\prime},-\underline{L}^{\prime}\right) \eta_{n}{ }^{\prime}\left(I^{\prime}\right)
$$

and from (10) and (11)

$$
b_{q}^{\prime}(m, \underline{K})=S_{q p^{\prime}}\left(m, \underline{K} ; n^{\prime}, \underline{L}^{\prime}\right), \quad b_{q}^{\prime \prime}(m, \underline{K})=S_{q p^{\prime \prime}}\left(m, \underline{K} ; n^{\prime \prime}, \underline{L}^{\prime \prime}\right) .
$$

Hence (after changing variables to get rid of the primes), we obtain the set of scattering reciprocity relations

$$
\eta_{m}(K) S_{p q}(m, \underline{K} ; n, \underline{L})=\eta_{n}(L) S_{q p}^{a}(n,-\underline{L} ; m,-\underline{K}) .
$$

In this case interchanging $S$ and $S^{a}$ yields no further information.

If the reader has followed through any one of the above exercises, he will have no trouble in showing

$$
S_{00}=S_{00}^{a}
$$

If we introduce the reciprocity dyadic

$$
\underline{\underline{n}}=\eta_{1} \underline{\kappa}_{1} \underline{\kappa}_{1}+\eta_{2} \underline{\kappa}_{2} \underline{\kappa}_{2}
$$

and use the definitions (1.3-3), then (for example) (12b) becomes

$$
\eta_{O} \underline{S}_{O q}(\underline{K})=\underline{\eta} \cdot \underline{S}_{q 0}^{\mathrm{a}}(-\underline{K})
$$

and (13) is summed up in

$$
\underline{\underline{n}}(\underline{K}) \cdot \underline{\underline{S}}_{\mathrm{pq}}(\underline{K}, \underline{L})=\underline{\underline{\tilde{S}}}_{q p}^{\mathrm{a}}(-\underline{\mathrm{L}},-\underline{K}) \cdot \underline{\underline{n}}(\underline{\mathrm{L}}),
$$

where $\underline{\underline{\eta}}(\underline{K})$ denotes $\underline{\underline{n}}$ as a function of $\underline{K}$ and the superposed " $"$ " denotes the transposed dyadic (obtained by transposing the elementary dyads involved). It is worth noting that the minus sign appearing between the members of (12b) does not appear in (16). The reciprocity dyadic is manifestly diagonal. in the $\underline{\kappa}_{1}$, $\underline{\kappa}_{2}$ basis; $(4.2-3)$ shows what happens when $\underline{n}$ is presented in the $\frac{e}{-x}, \frac{e}{y}$ basis. 
The Lorentz reciprocity relation seems to have been the quite generally preferred basis for the derivation of transmission or coupling equations (similar, at least in function, to our (2-15)) $[19,21,25,26]$. There is, however, no good reason why reciprocity should be invoked for that purpose: receiving characteristics can be defined analytically and operationally, independent of transmitting characteristics (as is done in this monograph). Nevertheless, it is of some interest that the generalized Lorentz relation (8), or, more conveniently in the plane-wave framework, the lemma (9), can be used to derive transmission equations without invoking (ordinary) reciprocity.

\subsection{Appendix B: Dissipative Characteristics of Media;}

\section{Comparison for Mutually Adjoint Media}

The concept that a medium may be "lossy," "lossless," or even "gainy" at a given point is familiar. We first need to give the mathematical expression of these qualitative properties of media. Then it will be easy to show that these properties are point-wise identical for mutually adjoint media.

According to properties of the complex Poynting's vector, dissipation or power loss per unit volume, $q(\underline{r})$, is given by

$$
q(\underline{r})=-\frac{1}{2} \operatorname{Re} \nabla \cdot(\underline{E} \times \underline{\bar{H}}) .
$$

In the medium described by (1.5-1), Maxwel1's equations require

$$
\begin{aligned}
& \nabla \times \underline{E}=i \omega(\underline{\mu} \cdot \underline{H}+\underline{\underline{\nu}} \cdot \underline{E}), \\
& \nabla \times \underline{H}=-i \omega(\underline{\underline{\varepsilon}} \cdot \underline{E}+\underline{\underline{\tau}} \cdot \underline{H}) .
\end{aligned}
$$


(Note that these equations are source-free in the sense that they are satisfied by $\underline{E} \equiv \underline{H} \equiv 0$.$) Using a standard vector identity:$ we combine (1) and (2) to obtain

$$
q(\underline{r})=-\frac{1}{2} \operatorname{Re}\left\{i \omega\left[\underline{\bar{H}} \cdot \underline{\underline{\mu}} \cdot \underline{H}-\underline{E} \cdot \underline{\underline{\varepsilon}} \cdot \underline{\bar{E}}+\underline{\bar{H}} \cdot\left(\underline{\underline{\nu}}-\underline{\underline{\tau}}^{*}\right) \cdot \underline{E}\right]\right\}, \quad(B-3)
$$

where the superscript "*" denotes the Hermitian conjugate. For our purpose it is convenient to rearrange this expression to read

$$
q(\underline{r})=\frac{\omega}{4}[\underline{\bar{E}}-i \underline{\bar{H}}] \cdot\left(\begin{array}{ll}
i\left(\underline{\underline{\varepsilon}}^{*}-\underline{\underline{\varepsilon}}\right) & \underline{\underline{\nu}} *-\underline{\underline{\tau}} \\
\underline{\underline{\nu}}-\underline{\underline{\tau}}^{*} & i(\underline{\underline{\mu}} *-\underline{\underline{\mu}})
\end{array}\right) \cdot\left(\begin{array}{c}
\underline{E} \\
i \underline{H}
\end{array}\right) .
$$

The dissipative properties of the medium are thus seen to be determined by properties of the Hermitian matrix

$$
\Lambda(\underline{r})=\left(\begin{array}{cc}
i(\underline{\underline{\varepsilon}} * \underline{\underline{\varepsilon}}) & \underline{\underline{\nu}} *-\underline{\underline{\tau}} \\
\underline{\underline{\nu}}-\underline{\underline{\tau}} * & i\left(\underline{\underline{\mu}}^{*}-\underline{\underline{\mu}}\right)
\end{array}\right),
$$

which we call the loss matrix for the medium. For the present purpose the characteristics of the medium at a given point are appropriately classified according to the values assumable by $\mathrm{q}(\underline{\mathrm{r}})$ as $\underline{E}$ and $\underline{H}$, considered independent and arbitrary, vary. possibilities are 1 isted and named in table B-1. 
Table B-1. Classification of Dissipative Characteristics.

\begin{tabular}{|c|c|c|}
\hline $\begin{array}{l}\text { Values assumed } \\
\text { by } \mathrm{q}(\underline{\mathrm{r}}) \\
\underline{\mathrm{r}} \mathrm{fixed}\end{array}$ & $\begin{array}{l}\text { Value class of } \\
\text { loss matrix at point } \underline{r}\end{array}$ & $\begin{array}{c}\text { Dissipative characteristics } \\
\text { of medium at point } \underline{r}\end{array}$ \\
\hline$>0$ & Positive definite & Unconditionally $\mid \begin{array}{l}\text { lossy or } \\
\text { passive }\end{array}$ \\
\hline$>0$ & Positive semidefinite & Conditionally \\
\hline$\equiv 0$ & Zero & Lossless, gainless or neutral \\
\hline$\overline{<} 0$ & Negative semidefinite & $\begin{array}{l}\text { Conditionally } \\
\text { gainy or } \\
\text { active }\end{array}$ \\
\hline$<0$ & Negative definite & Unconditionally \\
\hline$\frac{>}{<} 0$ & Indefinite & Indefinite \\
\hline
\end{tabular}

Note: In the semidefinite cases both the zero and the non-zero values are to be assumable, and in the indefinite case both positive and negative values are to be assumable.

There is no well-established terminology precisely fitting the physical properties being discussed. Terms generally chosen, and indeed the term "loss matrix," are biased by the tacit assumption that a medium norma11y is lossy. Thus, if the 1oss matrix happens to be the zero matrix, we would ordinarily say that the medium is lossless at the point in question. But the medium would also be gainless at the same point. Neither physical nor technological restrictions prevent the occurrence of negative energy loss, which might be called gain (cf. Brand [51], p. 150).

Mathematical criteria for deciding the value class of Hermitian matrices are given by Mirsky [52], Chapter 13. In the lossless case, which is physically important but not counted as defining a value class by Mirsky, the loss matrix must be the zero matrix; this in turn requires $\underline{\underline{\varepsilon}}=\underline{\underline{\varepsilon}} * \underline{\underline{\tau}}=\underline{\underline{\nu}} *$, and $\underline{\underline{\mu}}=\underline{\underline{\mu}}^{*}$. 
The medium adjoint to that described by (1.5-1) is defined and described by (1.5-2), according to which the adjoint expressions can be obtained by the replacements $\underline{\underline{\varepsilon}} \rightarrow \underline{\underline{\tilde{\varepsilon}}}, \underline{\underline{\tau}} \rightarrow-\underline{\underline{\underline{v}}}, \underline{\underline{\nu}} \rightarrow-\underline{\underline{\tau}}$, and $\underline{\underline{\mu}} \rightarrow \underline{\underline{\underline{\mu}}}$. For the loss matrix this yields

$$
\Lambda^{\mathrm{a}}(\underline{r})=\tilde{\Lambda}(\underline{r})
$$

i.e., $\Lambda^{\mathrm{a}}(\underline{r})$ is the transpose of $\Lambda(\underline{r})$. This relation is sufficiert to insure that the corresponding loss functions, $q(\underline{r})$ and $q^{a}(\underline{r})$, belong to the same value class (this can be seen, e.g., from the criteria given in Mirsky). We may say that the dissipative characteristics of a medium and its adjoint are the same, point by point. Whatever distribution of characteristics, including regions of active media (as in some antennas), is realized in one system will be realized in the adjoint system.

\subsection{Apperdix C: Generalized Joining Equations}

In the solution of the antenna-antenna interaction problems considered in section 2 we employed the relations connecting $\hat{b}_{1}$ and $\hat{a}_{1}$ of one antenna with $\hat{b}_{2}^{\prime}$ and $\hat{a}_{2}^{\prime}$ of the other. Our purpose in this appendix is to give a straightforward analytical derivation of these relations. They are derivable from the identity of the separate representations of the unique electromagnetic field (in the transmission path) asssociated with the separate antennas. (Essential notation is shown in figure 8.) The argument makes it clear that a reference plane is not sufficient--a reference point is required. This feature is unnoticeable in the discussion of the analogous problem of joining for waveguides (cf. (5.7-4) and 
(6.5-5) in Chapter I) and may account for some insecurity in intuitive carryover of waveguide concepts to the relations under discussion .

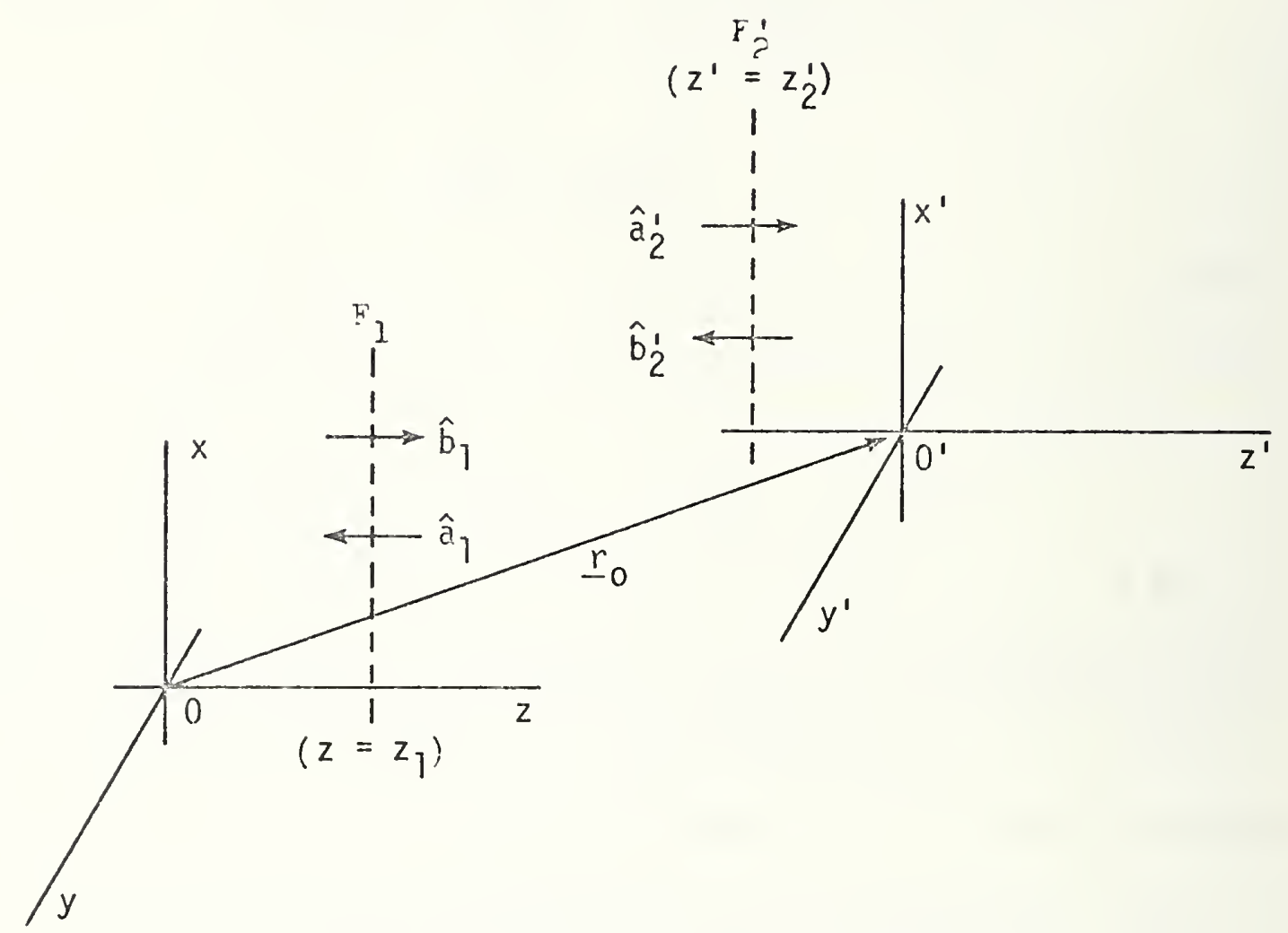

Figure 8. Parallel displacement of reference coordinate systems.

The spectral functions $\hat{a}_{1}, \hat{b}_{1}$ and the antenna scattering matrix $\hat{\mathrm{S}}_{\hat{\mathrm{S}}}$ are referred to the coordinate system $0 x y z ; \hat{a}_{2}^{\prime}, \hat{b}_{2}^{\prime}$, and $\hat{S}^{\prime}$ are referred to $0^{\prime} x^{\prime} y^{\prime} z^{\prime}$. Axes of Oxyz and $0^{\prime} x^{\prime} y^{\prime} z^{\prime}$ are respectively parallel; origin $0^{\prime}$ is at the point $\underline{r}_{0}$ in $0 x y z, \underline{r}_{0}=\underline{R}_{0}+z_{0} e_{z}, z_{0}>0$.

For convenience in the following application of $(1.2-9)$ we set

$$
\underline{E}_{q}(\underline{R}, z)+\underline{\underline{n}}^{-1} \cdot \underline{H}_{q}(\underline{R}, z) \times \underline{e}_{z}=4 \pi \underline{F}_{q}\left(\underline{R}, z, \underline{e}_{z}\right) .
$$

From (1.2-9) we then have 
Sec. 5.3

$$
\begin{aligned}
& b_{1}(m, \underline{K})=e^{-i \gamma z_{\underline{K}}} \cdot \int \underline{F}_{1}\left(\underline{R}, z, \underline{e}_{z}\right) e^{-i \underline{K} \cdot \underline{R}} d \underline{R} \\
& a_{2}^{\prime}(m, \underline{K})=e^{-i \gamma z^{\prime}} \underline{K}_{m} \cdot \int \underline{F} \underline{i}^{\prime}\left(\underline{R}^{\prime}, z^{\prime}, \underline{e}_{z}\right) e^{-i \underline{K} \cdot \underline{R}^{\prime}} d \underline{R}^{\prime}
\end{aligned}
$$

Now, when $\underline{R}, z$ and $\underline{R}^{\prime}, z^{\prime}$ represent the same physical point in space (between $z=z_{1}$ and $z^{\prime}=z_{2}^{\prime}$ ), then

$$
\begin{aligned}
\underline{F}_{1}\left(\underline{R}, z, \underline{e}_{z}\right) & \equiv \underline{F}_{2}^{\prime}\left(\underline{R}^{\prime}, z^{\prime}, \underline{e}_{z}\right), \\
\underline{R}^{\prime} & =\underline{R}-\underline{R}_{0}, \\
z^{\prime} & =z-z_{0} .
\end{aligned}
$$

Upon substituting all these relations in (3) and comparing the result with (2), we obtain

$$
a_{2}^{\prime}(m, \underline{K})=e^{i \underline{k}} \cdot \underline{r}_{O} b_{1}(m, \underline{K}),
$$

which is the first of the desired relations.

Again referring to (1) and $(1.2-9)$, we see that

$$
\begin{aligned}
& b_{2}^{\prime}(m, \underline{K})=e^{i \gamma z^{\prime}}{ }_{\underline{K}} \cdot \int \underline{F} \underline{2}^{\prime}\left(\underline{R}^{\prime}, z^{\prime},-\underline{e}-e^{-i K} \cdot \underline{R}^{\prime} d \underline{R}^{\prime}\right. \\
& a_{1}(m, \underline{K})=e^{i \gamma z_{\underline{K}}} \cdot \int \underline{F}_{1}\left(\underline{R}, z,-\underline{e}_{z}\right) e^{-i \underline{K} \cdot \underline{R}} d \underline{R}
\end{aligned}
$$

Corresponding to the first of (4) we now have

$$
\underline{F}_{1}\left(\underline{R}, z,-\underline{e}_{z}\right) \equiv \underline{F}_{2}^{\prime}\left(\underline{R}^{\prime}, z^{\prime},-\underline{e}_{z}\right)
$$

and of course we still have $\underline{R}^{\prime}=\underline{R}-\underline{R}_{O}$, etc. Substituting in (6) (say) and comparing the result with (7), we find

$$
a_{1}(m, \underline{K})=e^{-i \underline{k}-\underline{r}_{0}} b_{2}^{\prime}(m, \underline{K}),
$$

the second of the desired relations. 


\subsection{Appendix D: Representation of Planar Scanning Equation as a Convolution}

The transmission integral for planar scanning is, from (3.1-1),

$$
\Psi(\underline{P})=\int e^{i \underline{K} \cdot \underline{P}_{02}^{\prime}} S_{\underline{K}}^{\prime} \cdot \underline{S}_{10}(\underline{K}) e^{i \gamma d} d \underline{K} \cdot
$$

We should recall that here the characteristics of the transmitting and receiving antennas are referred to origins 0 and $0^{\prime}$, respectively, where $0^{\prime}$ is at the point $\underline{P}+\mathrm{de}_{-z}$ in the system Oxyz. For $\underline{S}_{10}$ we have, directly from $(1.3-6)$ with $q=1$, the Fourier transform representation

$$
\underline{S}_{10}(\underline{K})=\frac{e^{-i \gamma z_{1}}}{2 \pi a_{0}} \int \underline{E}_{1 t}\left(\underline{R}, z_{1}\right) e^{-i \underline{K} \cdot \underline{R}} d \underline{R} .
$$

We also need a suitable Fourier transform representation for $\underline{S}_{0}^{\prime}$. This result, which is of some interest in itself, is a lemma insofar as the main result of this appendix is concerned.

In connection with $(1.3-6)$ it was noted that we could have written Fourier transform representations in terms of the magnetic field rather than the electric field. We now write down a Fourier transform representation for the left-side transmitting characteristic of the antenna adjoint ${ }^{\mathrm{Dl}}$ to the given receiving antenna, choosing to exercise the option just mentioned.

$$
S_{20}^{a}(m, \underline{K})=\frac{e^{i \gamma z \dot{2}}}{2 \pi a_{0}^{\prime} \eta_{m}} \quad \frac{\kappa}{m} \cdot \int \underline{H}_{2}^{\prime a}\left(\underline{R}^{\prime}, z_{2}^{\prime}\right) \times \underline{n}_{2} e^{-i \underline{K} \cdot \underline{R}^{\prime}} d \underline{R}^{\prime} .
$$

Here $\underline{\mathrm{n}}_{2}=-\underline{\mathrm{e}}_{2}$, and $\underline{\mathrm{H}}_{2}^{\mathrm{a}}$ is the magnetic field produced on the plane $z^{\prime}=z_{2}^{\prime}$ by an incident wave of amplitude $a_{0}^{1}$ in the feed waveguide $\overline{D l}$ Of course, the receiving antenna may be reciprocal (=self-adjoint). 
Sec. 5.4

of the adjoint antenna. The adjoint reciprocity relation (1.5-4b), which reads

$$
\eta_{o} S_{o q}(m, K)=-\eta_{m}(K) S_{q o}^{a}(m,-\underline{K})
$$

enables us to eliminate the $\underline{K}$-dependent wave admittances and thus to write

$$
\underline{S}_{02}^{\prime}(\underline{K})=-\frac{e^{-i \gamma z i_{2}^{\prime}}}{2 \pi a_{0}^{\prime} n_{0}^{\prime}} \int \underline{H}_{2}^{\prime a}\left(\underline{R}^{\prime}, z_{2}^{\prime}\right) \times \underline{e}_{z} e^{+i K} \underline{R}^{\prime} d \underline{R}^{\prime} .
$$

This is the desired representation of $\underline{S}_{0}^{\prime} 2 \cdot$

We now let $z_{1}$ and $z_{2}^{\prime}$ represent the same plane (fjg. 9), so that we have

$$
z_{2}^{\prime}=z_{1}-d
$$

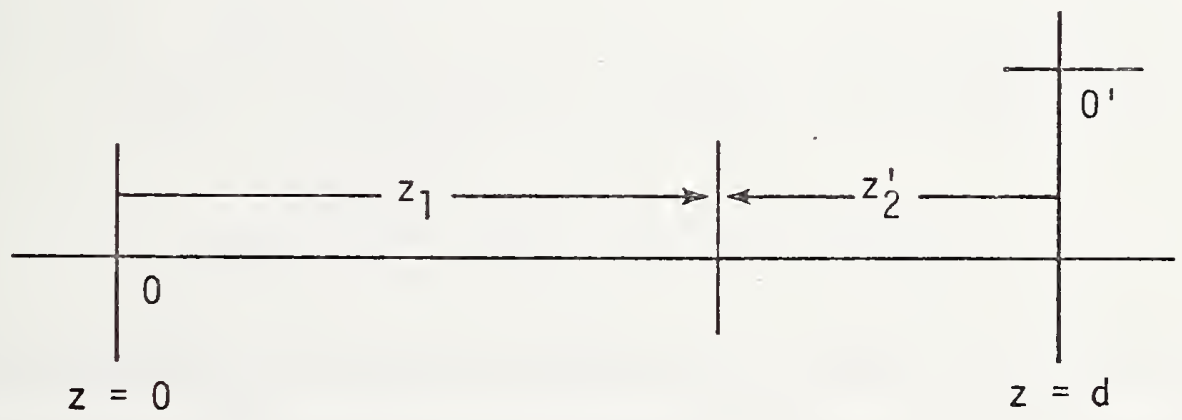

Figure 9. Relationship of $z_{1}, z_{2}^{\prime}$, and $d$.

Substituting (2) and (4) into (1) and using (5), we see that the exponentials containing $z_{1}$ and d cancel nicely. After a formally routine calculation, assisted (if need be) by (1.4-5), we arrive at the convolution integral

$$
\Psi(\underline{p})=\frac{-1}{a_{0}^{a} n_{0}^{\prime} n_{0}^{\prime}} \int\left[\underline{E}\left(\underline{R}, z_{1}\right) \underline{H}_{2}^{\prime a}\left(\underline{R}-\underline{p}, z_{1}-d\right) \underline{e}_{2}\right] d \underline{R} .
$$


It is interesting that the value of the integral is independent of the choice of the plane $z=z_{1}$ between the antennas. We know that this is so because the transmission integral in the original form (1) does not involve $z_{1}$ at all.

\subsection{Appendix E: Two-Dimensiona1, Spatial Sampling or Interpolation Theorem}

A variety of two-dimensional sampling theorems may be found in the literature $[42,53]$. The methods of derivation often used are unnecessarily complicated for our immediate purpose. For the convenience of the reader we sketch a simple derivation of the simple result that is obtained when perfect bandlimiting is assumed. For more general and more useful results see Petersen and Middleton [42].

With the abbreviation $\underline{S}_{0}^{\prime}(\underline{K}) \cdot \underline{S}_{10}(\underline{K}) \exp (i \gamma d)=f(\underline{K})$, the equation under discussion, $(3.1-1)$, becomes

$$
b_{0}^{\prime}(\underline{P})=a_{0} F^{\prime} \int f(\underline{K}) e^{i K} \cdot \underline{P} d \underline{K} .
$$

We have already noted that the mathematical requirement that $f(K)$ be "band limited" may be fulfilled extraordinarily and almost arbitrarily we1l. Let us therefore assume that nonzero values of $f(K)$ occur only in a finite region $K$ of wavenumber space. For simplicity, we take $K$ to be rectangular, bounded by the lines $k_{x}= \pm k_{1}, k_{y}= \pm k_{2}$. Then (1) may be written

$$
b_{0}^{\prime}(\underline{P})=a_{0} F^{\prime} \int_{-k_{2}}^{k_{2}} \int_{-k_{1}}^{k_{1}} f(K) e^{i K} \cdot \underline{p} d k_{x} d k_{y} \text {. }
$$


Sec. 5.5]

Further, $f(\underline{K})$ presumptively can be represented in $K$ by a double Fourier series, with periods $2 \mathrm{k}_{1}$ and $2 \mathrm{k}_{2}$ :

$$
f(\underline{K})=\sum_{r=-\infty}^{\infty} \sum_{s=-\infty}^{\infty} C_{r s} e^{-i \underline{K} \cdot \underline{P}} r s
$$

where the coefficients are given by the usual formula

$$
C_{r s}=\frac{1}{4 k_{1} k_{2}} \int_{-k_{2}}^{k_{2}} \int_{-k_{1}}^{k_{1}} f(\underline{K}) e^{i \underline{K} \cdot \underline{P}-r s} d k_{x} d k_{y}
$$

with

$$
\underline{\mathrm{p}}_{\mathrm{rs}}=\frac{\mathrm{r} \pi}{\mathrm{k}_{1}} \underline{\mathrm{e}}_{\mathrm{x}}+\frac{\mathrm{s} \pi}{\mathrm{k}_{2}} \underline{\mathrm{e}}_{\mathrm{y}} .
$$

Comparing (4) and (2), we see that the $C_{r s}$ are proportional to $\mathrm{b}_{\mathrm{o}}(\underline{\mathrm{P}})$ evaluated for $\underline{\mathrm{P}}=\underline{\mathrm{P}}_{\mathrm{rS}}$, so that (3) may be written

$$
f(\underline{K})=\frac{1}{4 k_{1} k_{2} a_{0} F^{\prime}} \sum_{r, s} b_{o}^{\prime}\left(\underline{P}_{r s}\right) e^{-\underline{i}-\underline{P}-r s} .
$$

Thus $f(\underline{K})$ is completely determined by the sampling of data at the discrete points $\underline{\mathrm{P}}_{\mathrm{rs}}$. This is the result used in (3.1-4).

The usual objective in sampling theory is the reconstruction of the sampled function. In the present instance this is easily accomplished by substituting (6) in (2) and integrating. The result is an expression involving cardinal functions and expressing $\mathrm{b}_{\mathrm{o}}^{\prime}($ P) at all points in terms of its values at the lattice points.

One should observe the reciprocal relation between the size of the region $K$ in wavenumber space and the size of the elementary cell in $x, y$ space. The smaller the former, the larger the latter-and the smaller the required density of sampling points in $x, y$ space. 


\section{REFERENCES}

[1] Kerns, D. M., and Dayhoff, E. S., Theory of diffraction in microwave interferometry, J. Res. Nat. Bur. Stds. (U.S.), 64B, 1-13 (Jan.-Mar. 1960).

[2] Kerns, D. M., Correction of near-field antenna measurements made with an arbitrary but known measuring antenna, Electron. Lett. 6, 346-347 (May 1970).

[3] Kerns, D. M., Scattering-matrix description and near-field measurements of electroacoustic transducers, J. Acoust. Soc. Am. 57, 497-507 (Feb. 1975).

[4] Cohen, M. H., Reciprocity theorem for anisotropic media, Proc. IRE (Correspondence) 43, 103 (Jan. 1955).

[5] Kerns, D. M., Generalized variational principles for electromagnetic vibrations; application to the theory of waveguide junctions, pp. 253-260, in Electromagnetic Theory and Antennas (Proc. 1962 Symp., Copenhagen, Denmark, E. C. Jordan, Ed., Pergamon Press, London, 1963).

[6] Wacker, P. F., Theory and numerical techniques for accurate extrapolation of near-zone antenna and scattering measurements, NBS Report (unpublished) (April 1972).

[7] Kerns, D. M., Plane-wave spectra and spectral coupling products for arbitrary radiating and scattering systems, NBS Report (unpublished) (Narch 1972).

[8] Baird, R. C., Newel1, A. C., Wacker, P. F., and Kerns, D. M., Recent experimental results in near-field antenna measurements, Electron. Lett. 6, 349-351 (Nay 1970).

[9] Newe11, A. C., and Kerns, D. M., Determination of both polarisation and power gain of antennas by a generalized 3-antenna measurement method, Electron. Lett. 7, 68-70, (Feb. 11, 1971).

[10] Joy, E. B., and paris, D. T., Spatial sampling and filtering in near-field measurements IEEE Trans. Antennas Prop. AP-20, 253-261 (May 1972).

[11] Newe11, A. C., Baird, R. C., and Wacker, P. F., Accurate measurement of antenna gain and polarization at reduced distances by an extrapolation technique, IleE Trans. Antennas prop. AP-21, 418-431 (1973). 
[12] Kanda, M., Accuracy considerations in the measurement of the power gain of a large microwave antenna, IEEE Trans. Antennas Prop. AP-23, 407-411 (1975).

[13] Yaghjian, A. D., Upper-bound errors in far-field antenna parameters determined from planar near-field measurements, Part 1: Analysis, Nat. Bur. Stds. (U.S.) Tech. Note 667 (1975).

[14] Newe11, A. C., Upper-bound errors in far-field antenna parameters determined from planar near-field measurements, Part 2: Analysis and computer simulation, Nat. Bur. Stds. (U.S.) Tech. Note 671 (1975).

[15] Johnson, R. C., Ecker, H. A., and Hollis, J. S., Determination of far-field antenna patterns from near-field measurements, Proc. IEEE 61, 1668-1694 (Dec. 1973).

[16] Woonton, G. A., On the measurement of diffraction fields, Proc. McGill Symp. Microwave Optics, pp. 347-350 (McGill University, Montreal, Quebec, Canada, 1953). B. S. Karasik, Ed., Air Force Cambridge Research Center, Cambridge, Mass. (1959).

[17] Dayhoff, E. S., Corrections in high accuracy Fresnel region microwave interferometry, Nat. Bur. Stds. (U.S.) Office of Basic Instrumentation Report (unpublished) (1956).

[18] Kerns, D. M., Wavelength correction in a microwave interferometer, URSI-IRE Meeting, Washington, D.C. (1957).

[19] Brown, J., A theoretical analysis of some errors in aerial measurements, Proc. Inst. Elec. Eng. 105C, 343-351 (Feb. 1958).

[20] Brown, J., A generalized form of the aerial reciprocity theorem, Proc. Inst. Elec. Eng. 105C, 472-475 (1958).

[21] Ju11, E. V., An investigation of near-field radiation patterns measured with large antennas, IRE Trans. Antennas Prop. AP-10, 363-369 (1962).

[22] Jull, E. V., The estimation of aerial radiation patterns from limited near-field measurements, Proc. IEE 110, 501-506 (1963).

[23] Brown, J., and Jull, E. V., The prediction of aerial radiation patterns from near-field measurements, Proc. Inst. Elec. Eng. 108B, 635-644 (Nov. 1961).

[24] Martin, W. W. Computation of antenna radiation pattern from near-field measurements, IEEE Trans. Antennas Prop. (Letter) AP-15, 316-318 (1967). 
[25] Jensen, F., Electromagnetic near-field far-field correlations, PhD Diss. (Technical University of Denmark, Lyngby, 1970).

[26] Leach, W. M., Jr., and Paris, D. T., probe compensated nearfield measurements on a cylinder, IEEE Trans. Antennas Prop. $\Lambda P-21,435-445$ (1.973).

[27] Wacker, P. F., Non-planar near-field measurements: spherical scaming, Nat. Bur. Stds. (U.S.) Report NBSIR 75-809 (1975).

[28] Kerns, D. M., Nnalytical techniques for the correction of near-field antenna measurements made with an arbitrary but known measuring antenna, URSI-IRE Neeting, Washington, D.C. (Apri1 29-May 2, 1963).

[29] Stratton, J. A., Electromagnetic Theory (McGraw-Hil1, 1941).

[30] Brillouin, L., The scattering cross-section of spheres for electromagnetic waves, J. Applied Physics, 20, No. 11, $1110-1124$ (1949).

[31] Born, M., and Wolf, E., Principles of Optics (pergamon, 1959).

[32] Messiah, A., Quantum Mechanics (North Holland, 1961).

[33] Van de Hulst, H. C., Light Scattering by Small Particles (John Wiley, 1957).

[34] Kerns, D. M., Plane-wave scattering-matrix and generalized reciprocity relations for antennas and scatterers, NBS Repoit (unpubiished) (June 1972).

[35] O'Dell, T. H., Magnetoelectrics--a new class of materials, Electronics and Power, 266-267 (August 1965).

[36] Condon, E. U., Theories of optical rotatory power, Rev. Mod. Phys. 9, 432-457 (Oct. 1937).

[37] Kong, Jin-Au, and Cheng, D. K., Modified reciprocity theorem for bianisotropic media, Proc. IEE (London) 117, 349-350 (FCb. 1970).

[38] Harrington, R. F., and Villeneuve, A. T., Reciprocity relationships for gyrotropic media, IRE Trans. Microwave Theory Tech. MTT-6, 308-310 (July 1958).

[39] Beckman, P., The Depolarization of Electromagnetic waves (Golem Press, Boulder, Colorado, 1968).

[40] Hollis, J. S., Lyon, T. J., and Clayton, L., Jr., (Eds.), Microwave Antenna Measuxements (Scientific Atlanta, Atlanta, Georgia, 1970). 
[41] Kerns, D. M., Antenna measurements with arbitrary antennas at arbitrary distances, Contribution in High-Frequency and Microwave Field-Strength Precision Measurement Seminar, NBS Report (unpublished) (1966).

[42] Petersen, D. P., and Middleton, D., Sampling and reconstruction of wave-number-limited functions in $\mathrm{N}$-dimensional Euclidean spaces, Information and Control 5, 279-323 (1962).

[43] Kerns, D. M., Analysis of symmetrical waveguide junctions, J. Res. Nat. Bur. Stds. (U.S.) 46, 267-282 (Apri1 1951).

[44] Kerns, D. M., New method of gain measurement using two identical antennas, Electron. Lett. 6, 348-349 (May 1970).

[45] Silver, S. (Ed), Microwave Antenna Theory and Design (Mass. Inst. Tech. Rad. Lab. Series, vol. 12, McGraw-Hil1, New York 1949).

[46] Bobber, R. J., Underwater Electroacoustic Measurements (Naval Res. Lab., Underwater Sound Reference Div., Orlando, Florida, $1970)$.

[47] MacLean, W. R., Absolute measurement of sound without a primary standard, J. Acoust. Soc. Am. 12, 140-146 (1940).

[48] Cook, R. K., Absolute pressure calibration of microphones, J. Acoust. Soc. Am. 12,415-420 (1941).

[49] Lanczos, C., Linear Differential Operators (D. Van Nostrand Co., Princeton, N.J., 1961).

[50] Courant, R., and Hilbert, D., Methods of Mathematical Physics (Interscience, New York, 1953).

[51] Brand, H., Schaltungslehre Linearer Mikrowellennetze, (S. Hirzel Verlag, Stuttgart, 1970).

[52] Mirsky, L., An Introduction to Linear Algebra, (Oxford University Press, Oxford, 1955).

[53] Goodman, J. W., Introduction to Fourier Optics (McGraw-Hill Book Company, New York, 1968).

[54] Kerns, D. M., Plane-wave scattering-matrix theory of antennas and antenna-antenna interactions: formulation and applications, J. Res. Nat. Bur. Stds. (U.S.), 80B, 5-51 (Jan.-Mar. 1976).

[55] Newel1, A. C., and Crawford, M. L., Planar near-field measurements on high performance array antennas, Nat. Bur. Stds. (U.S.) Report NBSIR 74-380 (July 1974). 
[56] Jackson, J. D., Classical Electrodynamics (John Wiley and Sons, 1962).

[57] Collin, R. E., and Zucker, F. J., (Eds.), Antenna Theory, Part I (McGraw-Hill, 1969). 


\section{CHAPTER III}

\section{THEORETICAL DEVELOPMENT AND ANALYTICAL EXAMPLES}

\section{INTRODUCTION}

In the preceding chapter a vector-dyadic form of the PISM for antennas was defined relative to $\underline{e}_{z}$-transverse components of incident and emergent spectra. For identification we call this the "uniplanar" form. This form reflects the original development progressing from waveguide-junction concepts, is convenient for the establishment of basic theorems, and is adequate for all purposes, including the establishment of the properties of the slightly modified equivalent form introduced in section 1 of the present chapter. The vector and dyadic scattering-matrix elements in this second form are transverse to the associated propagation vectors, and the form is thus called the "biplanar" form. It is convenient for most of the work of this chapter. (The biplanar form was first introduced systematically in an NBS Report [1]. ${ }^{1}$ )

In section 2 we show how very general classes of radiation and scattering problems can be reduced formally to the problem of prescribed currents in vacuo, and we obtain expressions for the PW spectra and for characteristic PW spectra in the reduced problem. This work is essentially that contained in section 2 of an NBS Report [2]. It furnishes the basis for the theoretical work in section 5 and for a series of simple analytical examples (including elementary electric and magnetic dipole antennas).

\footnotetext{
${ }_{\text {Figures in brackets indicate the literature references at the end of this }}$ chapter.
} 
Section 3 contains a restatement of some of the theory of minimum-scattering antemnas in the PWSM format. Minimumscattering antennas are introduced as lossless, conditionally non-scattering antennas. The term "conditionally non-scattering" is introduced as being more directly descriptive of a critical defining property of the minimum-scattering antennas. By endowing antennas with minimum scattering properties we create classes of simply and completely described antennas, which in turn are useful in the construction of analytical examples of coupled systems.

Section 4 defines classes of exactly solvable antennaantenna (or antenna-scatterer) interaction problems. Exactness means in particular that multiple reflections between antennas must be fully accounted for. Solvable means exactly reducible by "quadratures" to a finite system of linear algebraic equations. As might be expected, the criterion for solvability depends upon the scattering characteristics of the elements of the system considered. A sufficient condition for the solvability is that the scattering characteristic of one or the other of the elements of the system be degenerate (or of finite rank). A separable scattering characteristic, such as that of a minimum scattering antenna, represents the simplest (rank = 1) case. Although only elementary theory of integral equations is involved, the theory of this section (for rank > 1) is believed to be essentially new as far as antenna theory is concerned. Approximation of more general scattering characteristics by means of degenerate characteristics (as is possible in principle) implies approximate solvability for a larger class of problems. 
In section 5 expansions of asymptotic type are obtained for the transmission integral and for the reflection integral, together with a simple convergence criterion for the expansions. This work is an extension of the work in sections 4 and 5 of the NBS report [2]. For relationships to other work, indications of applications, and indeed for an adequate introduction to the character of the results, the reader is referred to section 5 itself.

In appendix $B$ we derive, as closely related corollaries of the expansion of the transmission integral in subsection 5.1, (a) the relation between asymptotic electric field and its spectrum and (b) an expansion theorem for the electric field of bounded scatterers. (The asymptotic relation in question is stated in (II-1.2-16) and it has been applied a good many times in this monograph.) These results are not new, but the method of derivation contains new elements. The resilts are obtained essentially by considering a transmission system consisting of a general radiating antenna and an ideal (elementary electric dipole) receiving antenna. Appendix B can be considered as an exercise illustrating some of the significance of the transmission integral and strengthening the theory of (II-1.2-16). 
1. SYSTEMATIC INTRODUCTION OF THE VECTOR-DYADIC PWSM OF THE "SECOND KIND"

In Chapter II a vector-dyadic form of the plane-wave scattering matrix for antennas is defined relative to $\underline{e}_{2}$-transverse components of the incident and emergent spectra. For immediate reference we repeat the defining equations, (II-1.3-4),

$$
\begin{aligned}
b_{0} & =S_{00} a_{0}+\sum_{p} \int \underline{S}_{0 p}(\underline{K}) \cdot \underline{A}_{p}(\underline{K}) d \underline{K}, \\
\underline{B}_{q}(\underline{K}) & =\underline{S}_{q 0}(\underline{K}) a_{0}+\sum_{p} \int \underline{S}_{q p}(\underline{K}, \underline{L}) \cdot \underline{A}_{p}(\underline{L}) d \underline{L} .
\end{aligned}
$$

For identification we may call this the "uniplanar" form, inasmuch as the scattering characteristic obeys the transversality relations

$$
\underline{e}_{z} \cdot \underline{\underline{S}}_{q p}(\underline{K}, \underline{L}) \equiv 0, \quad \underline{\underline{S}}_{q p}(\underline{K}, \underline{L}) \cdot \underline{e}_{z} \equiv 0
$$

and $\underline{S}_{-q o}$ and $\underline{S}_{o q}$ obey

$$
\underline{e}_{z} \cdot \underline{S}_{q 0}(\underline{K}) \equiv 0, \quad \underline{e}_{z} \cdot \underline{S}_{\mathrm{oq}}(\underline{K}) \equiv 0 .
$$

The form (1) reflects the original development progressing from waveguide junction concepts, is convenient for the establishment of basic theorems (having to do with power transfer, joining, and reciprocity), and is adequate for all purposes--including establishment of the properties of the slightly modified form that we are about to introduce. 
The second vector-dyadic form is defined with respect to what we have called the complete incident and emergent spectra (II-1.2-11), and in fact the defining equations are

$$
\begin{gathered}
b_{0}=s_{o o} a_{0}+\sum_{p} \int \underline{s}_{o p}(\underline{K}) \cdot \underline{a}_{p}(\underline{K}) d \underline{d}, \\
\underline{b}_{q}(\underline{K})=\underline{s}_{q 0}(\underline{K}) a_{0}+\sum_{p} \int \underline{s}_{q p}(\underline{K}, \underline{L}) \cdot \underline{a}_{p}(\underline{L}) d \underline{d} .
\end{gathered}
$$

For identification we may call this the "biplanar" form, ${ }^{2}$ since the scattering characteristics obey the transversality relations

$$
\underline{\mathrm{k}}^{ \pm} \cdot \underline{\underline{\mathrm{s}}}_{\mathrm{qp}}(\underline{\mathrm{K}}, \underline{\mathrm{L}}) \equiv 0, \quad \underline{\mathrm{s}} \mathrm{qp}(\underline{K}, \underline{\mathrm{L}}) \cdot \underline{\underline{l}}^{\bar{\mp}} \equiv 0
$$

and the receiving and transmitting characteristics obey

$$
\underline{\mathrm{k}}^{ \pm} \cdot \mathrm{s}_{\mathrm{qo}}(\underline{\mathrm{K}}) \equiv 0, \quad \underline{\mathrm{e}}^{\mp} \cdot \underline{\mathrm{s}}_{\mathrm{op}}(\underline{\mathrm{L}}) \equiv 0 \text {. }
$$

In some respects (3) is the most natural form for the antenna PWSM, but it is no more than equivalent to (1). It is convenient for the expression of the basic theorems and, more important, for the further development of the theory and for the construction of analytical examples.

Our primary task in this section is to derive the relations between the respective elements of the two vector-dyadic forms and to restate some of the basic theorems in terms of the newer entities. The basis for doing this is, of course, the relations

We use the term "biplanar" to identify a planar dyadic that is not in general uniplanar (cf. Gibbs-Wilson [3], p. 284). Transversality with respect to $\underline{e}_{e}$ is not inherent in (3) but is imposed as an auxiliary condition in a subsequent paragraph. 
between $\underline{A}_{q}$ and $\underline{a}_{q}$ and between $\underline{B}_{q}$ and $\underline{b}_{q}$ that are determined directly by the definitions in subsection II-1.2. (Perhaps we should remark that in essence our present task is exceedingly simple, and that part of it has already been accomplished unsystematically in subsection II-1.6. What difficulty there is arises from the need to keep track of the behavior of the unit vectors as $\underline{k}^{+} \rightarrow \underline{k}^{-}, \underline{K} \rightarrow-\underline{K}, \underline{k}^{+} \rightarrow-\underline{k}^{-}$, etc.) We start our systematic treatment by defining the dyadics

$$
\begin{aligned}
& \underline{\underline{\Omega}}(\underline{k})=\left(k_{z} / k\right) \underline{\kappa}_{1}(\underline{K}) \underline{e}_{\| l}(\underline{k})+\underline{k}_{2}(\underline{K}) \underline{e}_{\perp}(\underline{K}), \\
& \underline{x}(\underline{k})=\left(k / k_{z}\right) \underline{e}_{f}(\underline{k}) \underline{k}_{1}(\underline{K})+\underline{e}_{\perp}(\underline{K}) \underline{K}_{2}(\underline{K}),
\end{aligned}
$$

which possess the quasi-inverse properties

$$
\begin{aligned}
\underline{x}(\underline{k}) \cdot \underline{\underline{\Omega}}(\underline{k}) & =\underline{e}_{\|}(\underline{k}) \underline{e} \|(\underline{k})+\underline{e}_{1}(\underline{K}) \underline{e} \underline{1}(\underline{K}) \\
& \equiv \underline{\underline{\pi}}(\underline{k}), \\
\underline{\Omega}(\underline{k}) \cdot \underline{x}(\underline{k}) & =\underline{K}_{1}(\underline{K}) \underline{\underline{k}}_{1}(\underline{K})+\underline{k}_{2}(\underline{K}) \underline{K}_{2}(\underline{K}) \\
& \equiv \underline{\underline{\tau}} .
\end{aligned}
$$

(We continue to use the distinct notations for the congruent unit vectors $\underline{e}_{\perp}(\underline{K})$ and $\underline{K}_{2}(\underline{K})$, which do appear in somewhat different roles and contexts.) Note that $\underline{\underline{\tau}}=\underline{\underline{1}}-\underline{e}_{z} \underline{e}_{z}$ is a constant dyadic but $\underline{\underline{\pi}}(\underline{k})=\underline{\underline{1}}-\underline{e}_{k} \underline{e}_{k}$ is not. The products in (6) are plainly not unit dyadics; but they do have the key properties

$$
\underline{\underline{\tau}} \cdot \underline{\underline{\tau}}=\underline{\underline{\tau}}, \quad \underline{\underline{\pi}}(\underline{\mathrm{k}}) \cdot \underline{\underline{\pi}}(\underline{\mathrm{k}})=\underline{\underline{\pi}}(\underline{\mathrm{k}}),
$$


possession of which entitles them to be called idempotent (or projection) operators. In geometrical terms we can say that $\underline{\underline{\tau}}$ and $\underline{\underline{\pi}}(\mathrm{k})$ are equivalent to unit dyadics when (and only when) they operate on vectors that are already in or parallel to the planes defined by the respective dyadics.

With the aid of (5), the relations subsisting among $\Lambda_{q}$, $\underline{a}_{q}, \underline{B}_{q}$, and $\underline{b}_{q}$ may now be written

$$
\begin{aligned}
& \underline{B}_{-q}(\underline{K})=\underline{\underline{\Omega}}\left(\underline{k}^{ \pm}\right) \cdot \underline{b}_{q}(\underline{K}), \quad \underline{A_{q}}(\underline{K})=\underline{\underline{\Omega}}\left(\underline{k}^{\mp}\right) \cdot \underline{a} q(\underline{K}), \quad(1-8 \mathrm{a}) \\
& \underline{b}_{q}(\underline{K})=\underline{x}\left(\underline{k}^{ \pm}\right) \cdot \underline{B}-q(K), \quad \underline{a}-q(\underline{K})=\underline{x}\left(\underline{k}^{\mp}\right) \cdot \underline{A}_{q}(\underline{K}) \cdot(1-8 b)
\end{aligned}
$$

Here, as is repeatedly the case, due attention must be given to the "p,q $\vec{\leftarrow}$ sign-k $z$ " correlations. Next we use (8a) and (6a) to bring (1) into the form

$$
\begin{aligned}
& b_{0}=S_{0 o} a_{0}+\sum_{p} \int \underline{S}_{o p}(\underline{K}) \cdot \underline{\Omega}\left(\underline{k}^{\mp}\right) \cdot \underline{a}_{p}(\underline{K}) d \underline{K} \\
& \mathrm{~b}_{\mathrm{q}}(\underline{\mathrm{K}})=\underline{\chi}\left(\underline{\mathrm{K}}^{ \pm}\right) \cdot \underline{\mathrm{S}}_{\mathrm{qO}}(\underline{\mathrm{K}}) \mathrm{a}_{\mathrm{O}}+\sum_{\mathrm{p}} \int \underline{\chi}\left(\underline{\mathrm{k}}^{ \pm}\right) \cdot \underline{\underline{S}}_{\mathrm{qp}}(\underline{K}, \underline{\mathrm{L}}) \cdot \underline{\underline{\Omega}}\left(\underline{\ell}^{\mp}\right) \cdot \underline{\mathrm{a}}_{\mathrm{p}}(\underline{\mathrm{L}}) \mathrm{d} \underline{\mathrm{L}} \cdot
\end{aligned}
$$

Now, these equations must reproduce (3) for arbitrary $a_{0}$ and $\underline{a}_{p}$. Hence, we may at once conclude

$$
\underline{s}_{\mathrm{qO}}(\underline{K})=\underline{x}\left(\underline{\mathrm{k}}^{ \pm}\right) \cdot \underline{\mathrm{s}}_{\mathrm{qo}}(\underline{K})
$$

and, of course, $S_{o o}$ is the same in (1) and in (3). Since $\underline{a}_{p}(\underline{L})$ has no $\underline{e}_{\ell}$-component, comparison of the above form and (3) leaves a possible $\underline{e}_{\ell}$-component of $\underline{s}_{\mathrm{oq}}(\underline{L})$ and a possible dyadic component (of the form $\left.\underline{V}(\underline{K}, \underline{L}) \underline{e}_{\ell}\right)$ of $\underline{s}_{q p}(\underline{L})$ undefined. We may and do define these possible components to vanish; we then must have 


$$
\begin{aligned}
& \underline{s}_{\mathrm{oq}}(\underline{K})=\underline{\mathrm{S}}_{\mathrm{oq}}(\underline{K}) \cdot \underline{\underline{\Omega}}\left(\underline{\mathrm{k}}^{\mp}\right) \\
& \underline{\underline{s}}_{\mathrm{qp}}(\underline{K}, \underline{\mathrm{L}})=\underline{x}\left(\underline{\mathrm{k}}^{ \pm}\right) \cdot \underline{\underline{S}}_{\mathrm{qp}}(\underline{K}, \underline{L}) \cdot \underline{\underline{\Omega}}\left(\underline{l}^{\mp}\right) .
\end{aligned}
$$

Inspection of (9a) and (9b) shows that the $\underline{s}_{q o}$ and the $\underline{s}_{\text {oq }}$ introduced here are in fact identical to the identically labelled complete transmitting and complementary receiving characteristics introduced in $(1.6-3,-15)$ of II. The new scattering characteristic, $\underline{\underline{s}}_{q p}(\underline{K}, \underline{L})$, is similar to $\underline{s}_{q 0}(\underline{K})$ as a function of $q$ and $\underline{K}$ and similar to $\underline{s}_{o p}(\underline{L})$ as a function of $\mathrm{p}$ and $\underline{L}$. It is a biplanar dyadic, obeying the transversality equations (4a).

Equations (9) may be "inverted" to yield

$$
\begin{aligned}
& \underline{\mathrm{S}}_{\mathrm{qo}}(\underline{K})=\underline{\underline{\Omega}}\left(\underline{\mathrm{k}}^{ \pm}\right) \cdot \underline{\mathrm{s}}_{\mathrm{qo}}(\underline{K}) \\
& \underline{\mathrm{S}}_{\mathrm{oq}}(\underline{K})=\underline{\mathrm{s}}_{\mathrm{oq}}(\underline{\mathrm{K}}) \cdot \underline{\mathrm{X}}\left(\underline{\mathrm{k}}^{\mp}\right) \\
& \underline{\underline{S}}_{\mathrm{qp}}(\underline{K}, \underline{\mathrm{L}})=\underline{\underline{\Omega}}\left(\underline{\mathrm{k}}^{ \pm}\right) \cdot \underline{\underline{s}}_{\mathrm{qp}}(\underline{K}, \underline{\mathrm{L}}) \cdot x\left(\underline{e}^{\mp}\right) .
\end{aligned}
$$

This set of equations together with the set (9) may be regarded as expressing the equivalence of the formulations (1) and ( 3 ): either form may be derived from the other.

Using the " $\chi-\Omega$ calculus" it is easy to verify the identity of alternative expressions for the coupling products (which occur in the integrals of the Liouville-Neumann expansions). Thus one finds for the product occurring in the reflection integral $\Phi_{00}$,

$$
\underline{\mathrm{S}}_{01}(\underline{\mathrm{K}}) \cdot \underline{\underline{S}}_{22}^{\prime}(\underline{\mathrm{K}}, \underline{\mathrm{L}}) \cdot \underline{\mathrm{S}}_{10}(\underline{\mathrm{L}}) \equiv \underline{\mathrm{S}}_{01}(\underline{\mathrm{K}}) \cdot \underline{\underline{S}}_{22}^{\prime}(\underline{\mathrm{K}}, \underline{\mathrm{L}}) \cdot \underline{\mathrm{S}}_{10}(\underline{\mathrm{L}}) \cdot
$$


Similarly, for the product in the transmission integral, $\Psi_{0} 0^{\prime}$

$$
\underline{S}_{0}^{\prime}(\underline{K}) \cdot \underline{S}_{10}(\underline{K}) \equiv \underline{S}_{0}^{\prime}(\underline{K}) \cdot \underline{S}_{10}(\underline{K}) .
$$

This is consistent with (1.6-14a) in II; there, however, a different approach was adopted: essentially, the identity was postulated as a basis to obtain an ad hoc definition of $\underline{S}_{0 q}$. Warning: The unlikely looking product $\underline{S}_{01}(\underline{K}) \cdot \underline{S}_{10}(\underline{K})$, which occurs in the interferometer reflection integral given in [21], is not identically equal to $\underline{\mathrm{s}}_{01}(\underline{\mathrm{K}}) \cdot \underline{\mathrm{s}}_{10}(\underline{\mathrm{K}})$.

It is easily verified that the joining equations for the complete spectral vectors are

$$
\underline{a}_{2}^{i}(\underline{K})=\underline{b}_{1}(\underline{K}) e^{i \underline{k} \cdot \underline{r}}, \quad \underline{a}_{1}(\underline{K})=\underline{b}_{2}^{i}(\underline{K}) e^{-i \underline{k}}{ }^{-} \cdot \underline{r} .
$$

These are of the same form as the analogous equations (II-2-3). We now briefly restate expressions for power and for generalized (=adjoint) reciprocity in terms of elements of the biplanar form of scattering matrices defined in (3). Indeed, the power expression (II-1.4-8) becomes

$P_{q}=\frac{Y_{0}}{2 k} \int_{K<k}\left[\left|\underline{b}_{q}(\underline{K})\right|^{2}-\left|\underline{a}_{q}(\underline{K})\right|^{2}\right] \gamma d \underline{K}-\frac{Y_{0}}{k} \int_{K>k} R e\left[\underline{\underline{b}}(\underline{K}) \cdot \underline{a}_{q}(\underline{K}) \gamma\right] d \underline{K}$. $(1-14)$

This is somewhat simpler in form than the vector-dyadic form of (II-1.4-8) would be, but the complex basis vector $\underline{e}_{k}$ is implicit in the second integral in (14). The expression (II-A-16) for adjoint reciprocity of transmitting and receiving characteristics transforms to 


$$
\underline{s}_{O q}(\underline{K})=\frac{Y_{0}^{\gamma}}{\eta_{0} k} \underline{s}_{q 0}^{a}(-\underline{K})
$$

(a result already noted in (II-1.6-20)). The expression (II-A-17) for adjoint reciprocity of scattering characteristics takes on the appreciably simpler form

$$
\gamma_{K} \underline{\underline{s}}_{q p}(\underline{K}, \underline{L})=\gamma_{L} \stackrel{\tilde{\tilde{s}}}{\mathrm{a}}_{\mathrm{q} q}(-\mathrm{L},-\underline{K}) .
$$

Here $\gamma_{K}$ denotes $\gamma$ as a function of $K$ and the superposed " " denotes the transposed dyadic (obtained by transposing the elementary dyads involved). Detailed derivations of the last three equations are omitted, but we do furnish the following key relations: (a) The relation

$$
\underline{\underline{\Omega}}(\underline{k}) \cdot \underline{\underline{n}}(\underline{K}) \cdot \underline{\underline{\Omega}}(\underline{k})=Y_{O} \gamma \underline{\underline{\underline{I I}}}(\underline{\mathrm{k}}) / \mathrm{k}
$$

serves as a lemma for all three equations. (b) It is essential to observe that when $K>k$ (as in the second integral in (14)), then $\underline{k}^{\bar{q}}=\overline{\bar{k}^{ \pm}}$and this in turn implies

$$
\overline{\underline{\underline{\Omega}}}\left(\underline{k}^{ \pm}\right)=\underline{\underline{\Omega}}\left(\underline{k}^{\mp}\right) \text {. }
$$

(c) The dyadics $\underline{\underline{\Omega}}$ and $\underline{x}$ defined in (5) are of even parity under the inversion $\underline{k} \rightarrow-\underline{k}$; that is,

$$
\underline{\underline{\Omega}}(-\underline{k})=\underline{\underline{\Omega}}(\underline{k}), \quad \underline{x}(-\underline{k})=\underline{x}(\underline{k}) .
$$

This property is involved in the derivation of the reciprocity relations. 
2. SPECTRA OF ARBITRARY ANTENNAS, SCATTERERS, AND CURRENT SOURCES

2.1 Formal Reduction of Antenna and Scattering Problems to problems in Vacuo

We start with Maxwell's equations in the form

$$
\nabla \times \underline{E}-i \omega \underline{B}=0, \quad \nabla \times \underline{H}+i \omega \underline{D}=\underline{J}_{0} .
$$

These equations are essentially the same as (I-1-3); we have, however, redefined $\underline{D}$ to include $(i / \omega)$ times the induced current density vector, and we have distinguished the enforced current density with the subscript "o".

We shall account for the presence of material media, which will in general be inhomogeneous, lossy, and anisotropic, by means of the electric and magnetic polarization densities defined by

$$
\underline{\mathrm{p}}=\underline{\mathrm{D}}-\varepsilon_{0} \underline{\mathrm{E}}, \quad \underline{\mathrm{M}}=\left(\underline{B} / \mu_{0}\right)-\underline{H},
$$

where $\varepsilon_{0}$ and $\mu_{0}$ are "vacuum" values of permittivity and permeability, respectively.

In the formal results to be obtained here, all complications introduced by the properties of the media--which certainly would in general be formidable--remain implicit. It will not be necessary to make use of any explicit constitutive equations; in particular, (II-1.5-1) might be in force.

To obtain governing equations for a general radiating system (antenna), we assume that the primary source of the field is the 
enforced current density $\underline{J}_{0}$. Using (2) to eliminate $\underline{\mathrm{D}}$ and $\underline{\mathrm{H}}$ from (1), we obtain

$$
\nabla \times \underline{E}-i \omega \underline{B}=0, \quad \frac{I}{\mu_{0}} \nabla \times \underline{B}+i \omega \varepsilon_{0} \underline{E}=\underline{J}_{e},
$$

where $\underline{J}_{e} \equiv \underline{J}_{0}-i \omega \underline{P}+\nabla \times \underline{M}$. The effects of the material media are now represented in the modified current density $\underline{J}_{e}$, which must be considered to subsist in free space.

To obtain governing equations for a scattering system, we assume that the primary excitation of a structure of material media is an "incident" electromagnetic field generated by remote, fixed sources. Away from its sources the incident field satisfies

$$
\nabla \times \underline{E}_{0}-i \omega \underline{B}_{0}=0, \quad \frac{1}{\mu_{0}} \nabla \times \underline{B}_{0}+i \omega \varepsilon_{0} \underline{E}_{0}=0
$$

and the total field, incident plus induced, satisfies

$$
\nabla \times \underline{E}-i \omega \underline{B}=0, \quad \frac{1}{\mu_{0}} \nabla \times \underline{B}+i \omega \varepsilon_{0} \underline{E}=-i \omega \underline{P}+\nabla \times \underline{M}
$$

( $\underline{J}_{0}$ now being absent). Let the subscript " $i$ " identify induced fields; then $\underline{E}=\underline{E}_{0}+\underline{E}_{i}$ and $\underline{B}_{=} \underline{B}_{0}+\underline{B}_{i}$, and we have

$$
\begin{aligned}
& \nabla \times\left(\underline{E}_{0}+\underline{E}_{i}\right)-i \omega\left(\underline{B}_{0}+\underline{B}_{i}\right)=0 \\
& \nabla \times\left(\underline{B}_{0}+\underline{B}_{i}\right) / \mu_{0}+i \omega \varepsilon_{0}\left(\underline{E}_{0}+\underline{E}_{i}\right)=-i \omega \underline{P}+\nabla \times \underline{M} .
\end{aligned}
$$

Next we use (4) to eliminate the exciting field from the lefthand side of these equations and thus obtain 


$$
\nabla \times \underline{E}_{i}-i \omega \underline{B}_{i}=0, \quad \frac{1}{\mu_{0}} \nabla \times \underline{B}_{i}+i \omega \varepsilon_{0} \underline{E}_{i}=\underline{J}_{C}
$$

where in this case $\underline{J}_{C}=-i \omega \underline{P}+\nabla \times \underline{M}$.

We have obtained equations, (3) and (6), that are formally the same for the radiating problem and the scattering problem: There exists a current-density distribution $\underline{J}_{C}$, in general unknown, which, sustained in vacuum, would produce the $\underline{E}_{j}$ and $\underline{B}_{i}$ of the actual problem, both inside and outside the region of the material media involved (of course, $\underline{M}$ and $\underline{P}$ do vanish outside the region of the material media).

It secms worth noting that (2) permit one to climinate either $\underline{E}$ or $\underline{D}$ and, independently, either $\underline{B}$ or $\underline{H}$ (a total of four possibilities). The choice made yields supplementary currents of electric type only and has proved convenient and adequate for the problems considerd thus far.

2.2 Spectra of Prescribed Currents in Vacuo

Solutions of (2.1-3) or (2.1-6) are conveniently obtained with the aid of a suitable auxiliary vector II (which may be called a llertz vector). In fact if II satisfies the inhomogeneous wave equation

$$
\nabla^{2} \underline{I I}+k^{2} \underline{I I}=\underline{J}_{e} /\left(i \omega \varepsilon_{0}\right)
$$

(where $k^{2}=\omega^{2} \mu_{0} \varepsilon_{0}$ ), then $\underline{E}_{i}$ and $\underline{B}_{i}$ given by

$$
\underline{E}_{i}=\nabla \nabla \cdot \underline{I}+k^{2} \underline{I I}, \quad \underline{B}_{i}=-i \omega \mu_{0} \varepsilon_{0} \nabla \times \underline{I I}
$$


will satisfy (2.1-3) or (2.1-6). (At points where J evanishes $^{\text {valu }}$ we have also $\underline{E}_{i}=\nabla \times \nabla \times \underline{\Pi}$.j These equations are similar to ones given in Stratton [4], p. 430; we have modified them slightly to carry out our method of dealing with inhomogeneous media. II itself is given in terms of $\underline{J}_{e}$ by the well-known type of relation

$$
\underline{I I}(\underline{r})=-\frac{1}{4 \pi i \omega \varepsilon_{0}} \int \underline{J}_{e}\left(\underline{r}^{\prime}\right) \exp \left(i k\left|\underline{r}-\underline{r}^{\prime}\right|\right) /\left|\underline{r}-\underline{r}^{\prime}\right| \mathrm{d} \underline{r}^{\prime} .
$$

Here the volume integration goes over all non-zero $\mathrm{J}_{\mathrm{e}}$.

In order to introduce plane-wave spectra we need the planewave or Fourier-transform representation of the spherical wave $\exp (i k r) / r$ :

$$
\exp (i k r) / r=i /(2 \pi) \int \gamma^{-1} \exp (i \underline{K} \cdot \underline{R}+i \gamma|z|) d \underline{K},
$$

where $r=\left(x^{2}+y^{2}+z^{2}\right)^{\frac{1}{2}}$ and other notation is as previously established. In writing (4) the direction of the z-axis has been taken as a preferred direction, and the absolute-value sign is equivalent to the proviso

$$
\underline{\mathrm{k}}=\underline{\mathrm{k}}^{+}, \mathrm{z}>0 \text { and } \underline{\mathrm{k}}=\underline{\mathrm{k}}^{-}, \mathrm{z}<0 \text {, }
$$

where the superscripts "+" and "-" indicate the sign associated with $k_{z}$. Equation (4) is also well known ([5], p.239), although it is often rather well disguised ([4], p. 578). After substituting (4) into (3) and interchanging the order of integrations, we obtain the expression

$$
\underline{I}(\underline{r})=-\frac{1}{2 \pi} \int\left[e^{i \underline{k}} \underline{-r}^{+} \underline{U}_{1}(\underline{K}, z)+e^{i k} \underline{k}^{-} \cdot \underline{r}_{2}(\underline{K}, z)\right] \gamma^{-1} d \underline{K},
$$


where $\underline{U}_{1}$ and $\underline{U}_{2}$ are "cumulative spectra" given by

$$
\underline{U}_{1}(\underline{K}, z)=\frac{1}{4 \pi \varepsilon_{0} \omega} \int_{-\infty}^{z} d z^{\prime} e^{-i \gamma z^{\prime}} \int J_{-}\left(\underline{R}^{\prime}, z^{\prime}\right) e^{-i K \cdot \underline{R}^{\prime}} d \underline{R}^{\prime},
$$

and

$$
\underline{U}_{2}(\underline{K}, z)=\frac{1}{4 \pi \varepsilon_{0} \omega} \int_{z}^{\infty} d z^{\prime} e^{i \gamma z^{\prime}} \int \underline{J}_{C}\left(\underline{R}^{\prime}, z^{\prime}\right) e^{-i \underline{K} \cdot \underline{R}^{\prime}} d \underline{R}^{\prime} .
$$

For our purpose, (5), not (3), is now basic. Instead of differentiating (3) under the integral sign, we now must differentiate (5) under the integral sign to obtain $\underline{E}$ and $\underline{B}$ and to verify that II so represented satisfies (1). We expect that the process will be valid for mathematical representations of realistic current distributions. We assume that $\underline{J}_{C}$ is non-zero and integrable in a finite region $V$, and that the region $V$ itself may be multiply connected and re-entrant but not really pathological. Under these assumptions (5b) and ( $5 c$ ) become finite integrals and uniform convergence of the infinite integrals in (5a) is assured

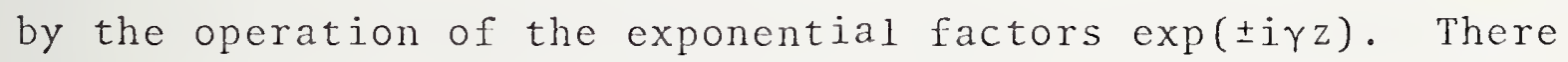
remains the effect of the discontinuities in $\exp (\mathrm{ikr}) / \mathrm{r}$ and in its representation (4). The course of the calculations involved is sketched in the following examples. (Although the details indicated are of considerable theoretical interest, not all are needed explicitly elsewhere in this monograph. What is needed is extracted in (7) and (8) below.) A11 differentiations with respect to $x$ and $y$ are straightforward; differentiations with respect to $z$ and interpretation of the resulting expressions require some care. 
Example 2.2-1: First derivatives. Show that

$$
e^{i \gamma z} \frac{\partial}{\partial z} \underline{U}_{1}(\underline{K}, z)+e^{-i \gamma z} \frac{\partial}{\partial z} \underline{U}_{2}(\underline{K}, z)=0
$$

This relation amounts to a lemma. It insures that all first-order $\nabla-$ operations are equivalent to the appropriate multiplications by ik under the integral signs. It also simplifies the calculation of second derivatives with respect to $z$.

Example 2.2-2: Second derivatives.

(a) Calculate $\nabla^{2} \underline{I I}$ and thus verify that $\underline{I}$ given by (5) satisfies (1). Let

$$
\nabla_{t}^{2}=\frac{\partial^{2}}{\partial_{x}^{2}}+\frac{\partial^{2}}{\partial y^{2}}
$$

Then

$$
\nabla_{t}^{2} \underline{I I}=\frac{1}{2 \pi} \int \mathrm{K}^{2}\{\} \mathrm{dK}
$$

where the braces denote the integrand of (5a). With the aid of the result of the preceding example, one finds

$$
\frac{\partial^{2}}{\partial z^{2}} \underline{I}=\frac{1}{2 \pi} \int \gamma^{2}\{\} \underline{d K}-i \frac{1}{2 \pi} \int\left(e^{i \underline{k}}+\underline{r} \frac{\partial}{\partial z} \underline{U}_{1}-e^{i \underline{k}-} \cdot \underline{r} \frac{\partial}{\partial z} \underline{U}_{2}\right) d \underline{K} .
$$

The second term on the right reduces to

$$
-\frac{i}{4 \pi^{2} E_{0} \omega} \int\left(e^{i K} \cdot \underline{R} \int \underline{J}_{e}\left(\underline{R}^{\prime}, z\right) e^{-i \underline{K} \cdot \underline{R}^{\prime}} d \underline{R}^{\prime}\right) d \underline{K}
$$


in which expression, interestingly enough, the two-dimensional Fourier transform of $\underline{J}_{e}(\underline{R}, z)$ is both done and undone, leaving us with $\underline{J}_{e}(\underline{r}) /\left(i \omega \varepsilon_{0}\right)$. Adding up the two contributions to $\nabla^{2} \underline{I}$, one finds that ( 1 ) is indeed satisfied.

(b) In very similar fashion, one finds for the evaluation of the expression (2) for $\underline{E}_{i}$,

$$
\begin{aligned}
& \underline{E}_{i}(\underline{r})=\frac{1}{2 \pi} \int\left(\underline{k}^{+} \underline{k}^{+}-k^{2} \underline{1}\right) \cdot \underline{U}-\underline{(K, z)} e^{i \underline{k}^{+}} \cdot \underline{r} \\
& \left.+\left(\underline{k^{-}} \underline{k}^{-}-k^{2} \underline{\underline{1}}\right) \cdot \underline{U}_{2}(\underline{k}, z) e^{i \underline{k}^{-} \cdot \underline{r}}\right) \gamma^{-1} d \underline{k} \\
& +\underline{e}_{z}-\underline{e}_{z} \cdot \underline{J}_{e} \underline{(r)} /\left(i \omega \varepsilon_{0}\right) \text {. }
\end{aligned}
$$

The last term in the above expression is closely related to some results recentIy obtained by Yaghjian [6] in a study of the mathematics of the dyadic Green's function for $\underline{E}$ produced by prescribed currents in vacuo. Yaghjian has shown that the conventional electric-field Green's function must be supplemented by an additive term that depends on the shape of the infinitesimal volume used to define the integral over the singularity of the Green's function. The above-mentioned term is just that obtained when the infinitesimal volume is chosen to be the slab between the planes $z-\varepsilon$ and $z+\varepsilon$ (where $\varepsilon$ eventually approaches zero).

Example 2.2-3: Consider a half-wave dipole centered at the origin, having the direction of the unit vector $\mathrm{e}_{\mathrm{p}}$, and carrying the current $I(s)=I_{c} \cos (k s)$, where $s$ is distance along the conductor measured from the center. Find the spectra of the electric fields in the regions $|z|>\frac{e}{z} \cdot e_{p} \lambda / 4$ to the "left" and to the "right" of the current distribution. Answer: 


$$
\underline{b}_{q}(\underline{k})=\frac{I_{c}}{2 \pi Y_{0}} \frac{\cos \left(\underline{k} \cdot \underline{e}_{p} \lambda / 4\right)}{k^{2}-\left(\underline{k} \cdot \underline{e}_{p}\right)^{2}} \underline{k} \times\left(\underline{k} \times \underline{e}_{p}\right) / \gamma .
$$

What is the spectral representation of $\underline{E}$ in the region $|z|<\underline{e}_{z} \cdot e_{p} \lambda / 4$ ?

In the absence of other evident needs and in the interest of very appreciable sjmplification, we shall consider further only cases in which the equivalent current density $\underline{J}_{e}$ is entirely to the "left" or entirely to the "right" of the regions of electromagnetic fields for which the corresponding spectra are desired. Under this restriction, $\underline{U}_{1}(\underline{K}, z)$ and $\underline{U}_{2}(\underline{K}, z)$ become independent of $z$ and we will henceforth use the notation $\underline{U}_{1}=\underline{U}_{1}(\underline{K}), \underline{U}_{2}=\underline{U}_{2}(\underline{K})$. (It may be observed that $\underline{U}_{1}(\underline{K})$ and $\underline{U}_{2}(\underline{K})$ are in fact evaluations of a single function of $\underline{k}$ for $\underline{k}=k^{+}$and $\underline{k}=\underline{k}^{-}$, respectively.) We now write down the pertinent formulas applicable in the two types of cases. The formulas may be obtained from (2) and (5), recognizing that the $\nabla$-operations and appropriate multiplication by ik under the integral sign will be fully equivalent. (The formulas may be obtained more directly from example $2(b)$, noting that the term containing $\underline{J}_{\mathrm{e}}$ will be absent.)

In the first case, $\underline{J}_{e}\left(\underline{r}^{\prime}\right) \equiv 0$ for $z^{\prime}>z_{1}$, say. Then for the induced electric field in $z>z_{1}$, we find from (2) and (5)

$$
\underline{E}_{i}(\underline{r})=\frac{1}{2 \pi} \int e^{i \underline{k}} \underline{ }^{+} \cdot \underline{r}\left[\underline{k}^{+} \underline{k}^{+} \cdot \underline{U}_{1}(\underline{K})-k^{2} \underline{u}_{1}(\underline{K})\right] \gamma^{-1} d \underline{k} ;(2.2-6)
$$


where

$$
\underline{U}_{1}(\underline{K})=1 /\left(4 \pi \varepsilon_{0} \omega\right) \int \underline{J}_{e}\left(\underline{r}^{\prime}\right) e^{-i \underline{\underline{k}}+\underline{r}^{\prime}} d \underline{r}^{\prime}
$$

and the volume integral goes over all non-zero $\underline{J}_{e}$. The angular spectrum $\underline{b}_{l i}(\underline{K})$ of the electric field represented by (6) is by definition, the coefficient of $\exp \left(\underline{i} \underline{k}^{+} \cdot \underline{r}\right) /(2 \pi)$ in (6). Thus

$$
\begin{aligned}
\underline{b}_{1 i}(\underline{K}) & =\left[\underline{k}^{+} \underline{k}^{+} \cdot \underline{U}_{1}(\underline{K})-k^{2} \underline{U}_{1}(\underline{K})\right] / \gamma \\
& =\underline{k}^{+} \times\left[\underline{k}^{+} \times \underline{U}_{1}(\underline{K})\right] / \gamma .
\end{aligned}
$$

Similarly, if $\underline{J}_{e}\left(\underline{r}^{\prime}\right) \equiv 0$ for $z^{\prime}<z_{2}$, then for the spectrum of the induced electric field in $z<z_{2}$, we find

$$
\underline{U}_{2}(\underline{K})=1 /\left(4 \pi \varepsilon_{0} \omega\right) \int \underline{J}_{e}\left(\underline{r}^{\prime}\right) e^{-i \underline{k}} \cdot \underline{r}^{\prime} d \underline{r}^{\prime}
$$

and

$$
\underline{\mathrm{b}}_{2 \mathrm{i}}(\underline{\mathrm{K}})=\underline{\mathrm{k}}^{-} \times\left[\underline{\mathrm{k}}^{-} \times \underline{\mathrm{U}}_{2}(\underline{\mathrm{K}})\right] / \gamma \text {. }
$$

The spectral functions $\underline{b}_{q i}(\underline{K})$ given by (7) and (8) are transverse in the sense that $\underline{k}^{+} \cdot \underline{b}_{1 i}(\underline{K})=0$ and $\underline{k}^{-} \cdot \underline{b}_{2 i}(\underline{k})=0--a$ property that can be attributed to the divergence condition $\nabla \cdot \underline{E}=0$ ( $\underline{c} \underline{\text {. }}$. $($ II $-1.2-2))$.

In equations (7) and (8) we have, for emphasis and greater clarity, shown the roles of $\underline{\mathrm{k}}^{+}$and $\underline{\mathrm{k}}^{-}$explicitly. We are not always equally explicit. The reader is reminded that the $\mathrm{p}, \mathrm{q} \underset{\leftarrow}{\lessgtr} \operatorname{sign-k_{z}}$ correlations are determinate (by the basic definitions) even when they are implicit. 
The successive vector multiplications occurring in (7b) and (8b) are, of course, equivalent to multiplication by a suitable dyadic. In fact, we have the identities

$$
\underline{k} \times(\underline{k} \times \underline{A})=\left(\underline{k k}-k^{2} \underline{\underline{1}}\right) \cdot \underline{A}=-k^{2} \underline{\underline{\pi}}(\underline{k}) \cdot \underline{A},
$$

where $\underline{A}$ is any vector, $\underline{\underline{1}}$ is the unit dyadic, and $\underline{\underline{\pi}}$ is the idempotent (or projection) operator already encountered in (1-6a). The recognition and use of the special properties of $\underline{\underline{\pi}}$ frequently simplify the vector algebra, especially in more complicated calculations occurring later.

Example 2.2-4:

(a) Use (7) and (8) to find the spectrum of a current element of moment $\underline{J}_{e} \mathrm{dV}$ located at the origin. This source may be regarded as an elementary electric dipole of electric moment $\underline{\underline{L}}=\underline{J}_{e} \mathrm{dV} /(-i \omega)$. Answer:

$$
\underline{\mathrm{b}}_{\mathrm{q}}(\underline{\mathrm{K}})=\left(4 \pi \varepsilon_{0} i\right)^{-1} \underline{\mathrm{k}} \times(\underline{\mathrm{k}} \times \underline{\mathrm{p}}) / \gamma .
$$

(b) The relation between spectrum and asymptotic $\underline{E}$ is given in (II-1.2-16b). Thus in particular the asymptotic E corresponding to the above spectrum is

$$
\underline{E}(\underline{r})_{\text {asymp. }}=-\frac{k^{2}}{4 \pi \varepsilon_{0}} e_{r} \times\left(\underline{e}_{r} \times \underline{p}\right) \frac{e^{i k r}}{r} .
$$

Conversely, the spectrum is determined by asymptotic E directly for $\mathrm{K}<\mathrm{k}$ and by analytic continuation for all values of $k_{x}$ and $k_{y^{*}}$ It is interesting that the spectrum may be determined from the asymptotic $\underline{E}$ alone and that the complete $\underline{E}$ may be determined by integration of the spectrum. 
(c) The E corresponding to the spectrum in part (a) of this example is given by (6) (evaluated for $\underline{k}=\underline{k}^{+}$and $\underline{k}=\underline{k}$ ) and is found to be

$$
\underline{E}(\underline{r})=\frac{1}{4 \pi \varepsilon_{0}}\left(\left(3 \underline{e}_{r} \cdot \underline{p} \underline{e}_{r}-\underline{p}\right)\left(\frac{1}{r^{3}}-\frac{i k}{r^{2}}\right)-\underline{e}_{r} \times\left(\underline{e}_{r} \times \underline{p}\right) \frac{k^{2}}{r}\right) e^{i k r} .
$$

Since this is the electric field of an elementary electric dipole, the result is easily found. See, e.g., [4], p. 435. Alternatively, one may evaluate the seven closely-related integrals involved. The simplest of. these is well known and is given in (4). The others may be evaluated by differentiation under the integral sign or in other ways.

(d) For the total power radiated one finds from the formula in example II-1.4-1 the well-known result

$$
P_{1}+P_{2}=\frac{k^{2}|\omega \underline{p}|^{2}}{12 \pi Y}
$$

Example 2.2-5:

(a) Find $\underline{U}_{\mathrm{q}}$ and hence the spectrum of a uniform current $I_{0}$ in a small circular loop of area A, centered at the point $\underline{r}$. Let the positive direction of the current and the unit vector $e_{m}$ normal to the area be related by the right-hand rule. This current distribution is the "Amperian current" equivalent to an (oscillating) magnetic dipole of moment $\underline{m}=A I_{0} e_{m}^{3}$ Answers:

$$
\begin{aligned}
& \underline{U}_{q}(\underline{k})=\frac{i \underline{k} \times \underline{m}}{4 \pi \varepsilon \omega} e^{-i \underline{k} \cdot \underline{r}_{0}} \\
& \underline{b}_{q}(\underline{k})=\frac{-i k^{2}(\underline{k} \times \underline{m})}{4 \pi \varepsilon \omega \gamma} e^{-i \underline{k} \cdot \underline{r}_{0}},
\end{aligned}
$$

\section{We follow stratton [4], p. 438, in this definition of magnetic moment.}

Schelkunoff $[7], \mathrm{p} .70$, would have $\underline{m}=\mu_{0} \mathrm{AI}_{0} \mathrm{e}_{\mathrm{m}}$. 
(b) The electric field corresponding to the current and the spectrum in part (a) of this example with $\underline{r}_{0}=0$ is

$$
\underline{E}(\underline{r})=\frac{k^{2}}{4 \pi Y_{0}}\left(\frac{1}{r}+\frac{i}{k r}\right) \underline{m} \times \underline{e}_{r} e^{i k r}
$$

(c) For the total power radiated one finds in this case the we11-known result

$$
\mathrm{P}_{1}+\mathrm{P}_{2}=\frac{\mathrm{k}^{4}|\underline{\mathrm{m}}|^{2}}{12 \pi \mathrm{Y}_{0}}
$$

In this and in the preceding example $\mathrm{P}_{1}=\mathrm{P}_{2}$ for any orientation of the dipoles.

The spectra for E calculated above (in the general formulas as well as in the examples) require only the proper setting and the proper normalization to be identifiable as characteristic transmitting or scattering functions, directly in accordance with the definitions of these quantities.

Suppose we wish to apply (7) and (8) to an antenna in its transmitting mode. The primary excitation of the antenna may then be represented in $(2.1-3)$ by a suitable prescribed surface electric current on $S_{0}, 4$ and we have very simply

$$
\underline{s}_{q 0}(\underline{K})=\underline{b}_{q}(\underline{K}) / a_{0},
$$

${ }^{4}$ Specifically, the generator enf may be represented by a surface electric current on $S_{0}$ equal to $2 \eta_{0} b_{G}\left(1+\Gamma_{G}\right)^{-1} \underline{n}_{0} \times \underline{h}_{0}(\underline{r})$, where $\Gamma_{G}$ is the reflection coefficient and $b_{G}$ the generated wave of the equivalent source. For other notation and for related concepts see subsections II-1.1 and I-5.7. 
where $\underline{b}_{q}$ is given by (7) and (8), and of course $\underline{a}_{q} \equiv 0$ is understood. This normalization is actually carried out in some simple analytical examples given below.

To apply (7) and (8) to the representation of scattering characteristics, we suppose the primary excitation of a passive antenna (or other passive scattering object) is an incident spatial plane wave having $\underline{E}=\underline{\alpha}_{p}(\underline{L}) \exp (\underline{i} \underline{\ell} \cdot \underline{r}) / 2 \pi$, and let $\underline{\mathrm{b}}_{\mathrm{qs}}(\underline{\mathrm{K}})$ (given by (7) and (8)) denote the spectra of the corresponding scattered electric fields in the regions to the right and to the left of the scatterer. (See figure 1 and example 7, below.) We recall the necessity of distinguishing total and true or bona fide trans-scattering (cf. (II-1.3-14) and related discussion), and we notice that (7) and (8) inherently yield spectra of the true scattered fields. It will be useful to have a dyadic operator directly characterizing actual scattering processes, and so we define $\underline{\underline{t}}_{q p}(\underline{K}, \underline{L})$ by postulating as identities the relation

$$
\underline{\mathrm{b}}_{\mathrm{qS}}(\underline{K}) \equiv \underline{\underline{t}}_{q p}(\underline{K}, \underline{\mathrm{L}}) \cdot \underline{\alpha}_{\mathrm{p}}(\underline{\mathrm{L}})
$$

and the auxiliary requirement $\underline{\underline{t}}_{\mathrm{qp}}(\underline{K}, \underline{\mathrm{L}}) \cdot \underline{\mathrm{e}}_{2}=0(\underline{\mathrm{c} f} \cdot(1-3))$. Since PWSM cis-scattering characteristics always represent true scattering, we may make the identification

$$
\underline{\underline{s}} q q(\underline{K}, \underline{L}) \equiv \underline{t}_{q q}(\underline{K}, \underline{L}) .
$$


The PWSM trans-scattering characteristics, in accordance with (II-1.3-14), are given by

$$
\underline{\underline{s}}_{q p}(\underline{K}, \underline{L}) \equiv \underline{\underline{t}}_{q p}(\underline{K}, \underline{L})+\underline{\underline{\pi}}(\underline{k}) \delta(\underline{K}-\underline{L}) \cdot \quad(p \neq q)
$$

Here the second term on the right, with $\underline{\underline{\pi}}(\underline{\mathrm{k}})$ defined in $(1-6 \mathrm{a})$ and again in (9), is equivalent to the identity for incident spectra and preserves the property $\underline{\underline{s}}_{q p}\left(\underline{K}, \underline{L}_{L}\right) \cdot \underline{e}_{l}=0$.

Example 2.2-6: The differential, bistatic, scattering cross-section defined in (II-1.6-23) is expressible in the form

$$
\sigma_{p q}(\underline{K}, \underline{L})=1.6 \pi^{3} \gamma_{K}^{2}\left|\underline{t} \underset{p q}{ }(\underline{K}, \underline{L}) \cdot \underline{\alpha}_{q}(\underline{L})\right|^{2} /\left|\underline{\alpha}_{q}(\underline{L})\right|^{2},
$$

where $\stackrel{t_{p q}}{=}$ is the scattering characteristic defined in the preceding paragraph.

Example 2.2-7: $\quad$ tqp for a small dielectric sphere. The scattered field of a small dielectric sphere is in the first approximation that of an electric dipole whose moment is $\underline{\underline{\alpha}} \cdot \underline{E}$, where $\underline{\underline{\alpha}}$ is the polarizability

$$
\underline{\underline{\alpha}}=4 \pi \varepsilon_{0} a^{3} \frac{\varepsilon_{1}-\varepsilon_{0}}{\varepsilon_{1}+2 \varepsilon_{0}} \underline{\underline{1}} .
$$

Here $a$ is the radius of the sphere, $\varepsilon_{1}$ the dielectric constant, and $\underline{\underline{1}}$ is the unit dyadic. The coefficient in the above expression may be found, e.g., in Stratton [4], p. 572. Using the result in example (4a), the definition (11), and assuming the sphere centered at the origin, one finds

$$
\underline{t}_{q p}(\underline{K}, \underline{L})=\frac{i k^{2} a^{3}}{2 \pi \gamma_{K}} \frac{\varepsilon_{1}-\varepsilon_{O}}{\varepsilon_{1}+2 \varepsilon_{0}} \underline{\underline{\pi}}(\underline{k}) \cdot \underline{\underline{\pi}}(\ell) .
$$


As a function of $\mathrm{q}$ and $\underline{\mathrm{K}}$ this yields the spectra of the scattered electric fields in the regions $z>a$ and $z<-a$.

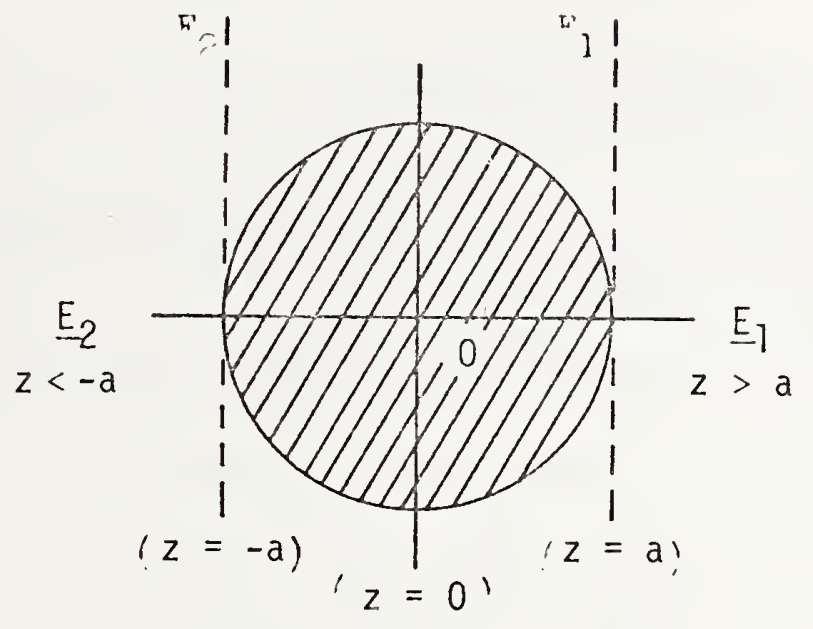

Figure 1. Regions of $\underline{E}_{1}$ and $\underline{E}_{2}$ for the sphere.

Example 2.2-8: Consider a lossless, reciprocal antenna whose radiated field is identical to that of an elementary electric dipole situated at the point whose radius vector is $\underline{r}_{0}$.

(a) The transmitting characteristic is found by normalizing the spectrum found in example 4 with respect to $a_{0}$ :

$$
\underline{s}_{q_{0}}(\underline{k})=\frac{1}{4 \pi \varepsilon \gamma}\left(\frac{p}{i a_{0}}\right) \underline{k} \times\left(\underline{k} \times \underline{e}_{p}\right) e^{-i \underline{k} \cdot \underline{r} 0},
$$

where $\underline{p}=p \underline{e}_{p}, \underline{p}$ is taken cophasal, and $\underline{e}_{p}$ is a real unit vector.

(b) Note that $\mathrm{p} / \mathrm{a}_{0}$ is a constant independent of $\mathrm{a}_{\mathrm{o}}$; for convenience and some emphasis we introduce the notation $\mathrm{N}_{\mathrm{e}}=\mathrm{p} /\left(\mathrm{ia}{ }_{\mathrm{o}}\right)$. By equating net input power to radiated power we find 


$$
\left|\mathrm{N}_{e}\right|^{2}=\frac{6 \pi n_{0}\left(1-\left|s_{o O}\right|^{2}\right)}{Y_{0} \omega^{4} \mu^{2}}
$$

(c) From (a), (b), and (1.6-6) in II, we find the power gain function

$$
G_{q}(\underline{k})=\frac{3}{2} \sin ^{2}\left(\underline{e}_{p}, \underline{k}\right) .
$$

Here $\left(e_{p}, \underline{k}\right)$ denotes the angle between the real vectors $e_{p}$ and $\underline{k}$. The power gain function is, of course, independent of $\underline{r}_{0}$.

(d) Using reciprocity in the form (II-1.6-20a), we find for the receiving characteristic of the antenna under consideration

$$
\underline{s}_{\mathrm{Oq}}(\underline{K})=\frac{\mathrm{Y}_{\mathrm{O}} \mathrm{e}_{\mathrm{e}}}{4 \pi \varepsilon k \eta_{\mathrm{o}}} \underline{\mathrm{k}} \times\left(\underline{\mathrm{k}} \times \underline{\mathrm{e}}_{\mathrm{p}}\right) \mathrm{e}^{+\underline{i} \underline{k} \cdot \underline{\mathrm{r}}_{\mathrm{O}}} .
$$

(e) With the antenna placed in an electromagnetic field $\underline{E}, \underline{H}$ and provided with a passive load having reflection coefficient $\Gamma_{L}$, the received wave-amplitude is

$$
b_{0}=C_{e} \stackrel{e}{p}_{p} \cdot \underline{E}\left(\underline{r}_{0}\right) .
$$

With the aid of the result in Appendix $A$, the value of $C_{e}$ is found to be

$$
C_{e}=-\frac{\omega N}{e^{F}},
$$

where $F=1 /\left(1-S_{o O} \Gamma_{L}\right)$. For the squared magnitude we find the more explicit form

$$
\left|\mathrm{C}_{\mathrm{e}}\right|^{2}=\frac{3 \lambda^{2} \mathrm{Y}_{\mathrm{O}}}{8 \pi n_{\mathrm{o}}} \frac{1-\left|\mathrm{S}_{\mathrm{oo}}\right|^{2}}{T 1-\Gamma_{\mathrm{L}} \mathrm{S}_{\mathrm{oO}}} T^{2}
$$

using the value of $\left|\mathrm{N}_{\mathrm{e}}\right|$. 
(f) From the preceding result one can calculate the received power, and in particular the available received power, which can be written

$$
\mathrm{P}_{\mathrm{A}, \mathrm{rec}}=\frac{3 \lambda^{2}}{8 \pi} \frac{\mathrm{Y}_{\mathrm{o}}}{2}\left|\underline{\mathrm{e}}_{\mathrm{p}} \cdot \underline{\mathrm{E}}\left(\underline{\mathrm{r}}_{\mathrm{o}}\right)\right|^{2} .
$$

(g) We now have at hand three ways of obtaining the effective area: by reciprocity (II-1.6-21a) from the power gain, by substitution of the result in part (d) into the formula (II-1.6-19), and more basically by reference to the defining equation (II-1.6-8). By any method the result is

$$
\sigma_{q}(\underline{k})=\frac{3 \lambda^{2}}{8 \pi} \sin ^{2}(\underline{e}, \underline{k}) .
$$

Here $\left(\mathrm{e}_{\mathrm{p}}, \mathrm{k}\right)$ denotes the angle between the real vector $\underline{e}_{\mathrm{p}}$ and the real propagation vector $k$ of the incident plane wave. (Note that the assumption of an incident plane wave has not hitherto been involved.) The maximum possible effective area is seen to have the well-known value $3 \lambda^{2} / 8 \pi$.

Example 2.2-9: Consider a lossless, reciprocal antenna whose radiated field is identical to that of an infinitesimal current loop (or, equivalently, to that of an elementary magnetic dipole) located at the point $\underline{r}=\underline{r}_{0}$.

(a) Normalizing the spectrum found in example 5 with respect to $a_{0}$, we find the transmitting characteristic

$$
\underline{s}_{q 0}(\underline{K})=-\left(\frac{m}{a_{0}}\right) \frac{i k^{2}(\underline{k} \times \underline{e})}{4 \pi \varepsilon e \omega \gamma} e^{-i k \cdot \underline{r}_{0}},
$$

where $\underline{m}=m e, m$ is cophasal, and $e_{-m}$ is a real unit vector. 
(b) We observe that $\mathrm{m} / \mathrm{a}_{0}$ is a constant independent of $\mathrm{a}_{0}$, set $\mathrm{N}_{\mathrm{m}}=\mathrm{m} / \mathrm{a}_{\mathrm{O}}$, and by equating net input power to radiated power find

$$
\left|\mathrm{N}_{\mathrm{m}}\right|^{2}=6 \pi \mathrm{Y}_{\mathrm{o}} \eta_{\mathrm{o}}\left(1-\left|\mathrm{S}_{\mathrm{O} O}\right|^{2}\right) / \mathrm{k}^{4}
$$

(c) The power gain function is found from (a), (b) and (II-1.6-6) to be

$$
G_{q}(\underline{K})=\frac{3}{2} \sin ^{2}(\underline{e}, \underline{k})
$$

Here $\left(e_{m}, k\right)$ denotes the angle between the real vectors $e_{-m}$ and $\underline{k}$. This result is properly independent of $\underline{r}_{0}$.

(d) Reciprocity in the form (II-1.6-20a) yields for the receiving characteristic of the antenna being considered

$$
\underline{s}_{\mathrm{oq}}(\underline{k})=\frac{\mathrm{N}_{\mathrm{m}} i\left(\underline{k} \times \underline{\mathrm{e}}_{\mathrm{m}}\right)}{4 \pi n_{0}} \mathrm{e}^{+i \underline{k} \cdot \underline{\mathrm{r}} \mathrm{o}} .
$$

(e) With the antenna placed in an electromagnetic field $\underline{E}$, $\underline{H}$ and provided with a passive load having reflection coefficient $\Gamma_{L}$, the received wave-amplitude is

$$
b_{0}=C_{m} e \cdot m \cdot \underline{H}\left(r_{0}\right) .
$$

Here

$$
\mathrm{C}_{\mathrm{m}}=-\frac{i k \mathrm{~N}_{\mathrm{m}} \mathrm{F}}{2 \pi_{\mathrm{O}} \mathrm{Y}_{\mathrm{o}}}
$$

where $\mathrm{F}$ is the mismatch factor defined in the preceding example, and

$$
\left|\mathrm{C}_{\mathrm{m}}\right|^{2}=\frac{3 \lambda^{2}}{8 \pi} \frac{1}{\eta_{\mathrm{O}} \mathrm{Y}_{\mathrm{O}}} \frac{\left(1-\left|\mathrm{S}_{\mathrm{OO}}\right|^{2}\right)}{\left|1-\mathrm{S}_{\mathrm{OO}} \Gamma_{\mathrm{L}}\right|^{2}} .
$$


The derivation of these results is similar to the derivation in the corresponding electric dipole antenna problem.

(f) Calculating received power and then setting $\Gamma_{L}=\bar{S}_{O 0}$, one finds for the available power at the receiving antenna terminal,

$$
\mathrm{P}_{\mathrm{A}, \mathrm{rec}}=\frac{3 \lambda^{2}}{8 \pi} \frac{1}{2 Y_{0}}\left|\underline{e}_{m} \cdot \underline{H}\left(\underline{r}_{0}\right)\right|^{2} .
$$

(g) Effective area is perhaps most instructively found by application of the defining equation (II-1.6-8). The effective area is

$$
\sigma_{q}(\underline{K})=\frac{3 \lambda^{2}}{8 \pi} \sin ^{2}\left(\underline{e}_{-m}, \underline{k}\right)
$$

where $\left(\underline{e}_{m}, \underline{k}\right)$ denotes the angle between the real vector $e_{-m}$ and the real propagation vector $\underline{k}$ of an incident plane wave. Compare the result in example 8.

With data now available we are in a position to write down (and evaluate) transmission integrals for several pairings of elementary dipole antennas. The case of coupled elementary electric dipole antennas is of most interest and importance, and will be of direct benefit in derivations later in this chapter. This case will be considered in the next example. The general procedure set up in section II-2 will be followed (in particular in the matter of using two fiducial coordinate systems); and, to adapt the example to the later needs, both antennas are located away from the origins in their respective coordinate systems. 
Example 2.2-10: Transmission integral for coupled elementary electric dipole antennas.

(a) Let the transmitting antenna be at $\underline{r}$ in Oxyz, the receiving antenna at $\underline{x}^{\prime}$ in $O^{\prime} x^{\prime} y^{\prime} z^{\prime}\left(O^{\prime}\right.$ being at $\underline{r}_{0}$ in $\left.O x y z\right)$, consider transmission from left to right, take data from example 8, and form the expression for the transmission integral (II-2-15).

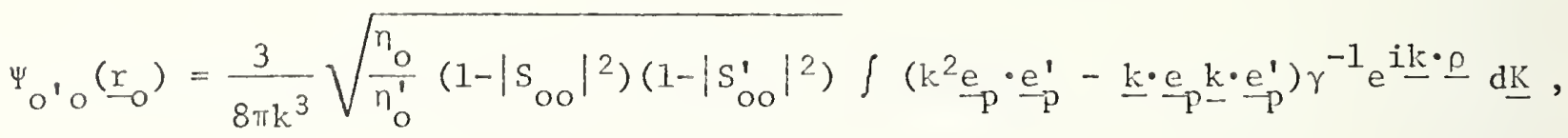
where $\underline{\rho} \equiv \underline{r}_{0}+\underline{r}^{\prime}-\underline{r}$. Thus far the phases of $N_{e}$ and $N_{e}^{\prime}$ are indeterminate; we have written the above expression for positive real $\mathrm{N}_{\mathrm{e}}$ and $\mathrm{N}_{\mathrm{e}}^{\prime}$. The integrations are, of course, tractable, being the same as those implicit in example $4(c) .^{5}$

(b) With the integrals in the above expression evaluated, we have

$$
\Psi_{0}^{\prime}{ }_{0}\left(\underline{r}_{0}\right)=\operatorname{Cf}\left(\underline{e}_{p}, \underline{e}_{p}^{\prime}, \underline{\rho}\right)
$$

where $f$ is the "dipole-dipole coupling function" for elementary electric dipoles,

$$
\begin{aligned}
& f\left(\underline{e}_{p}, \underline{e}_{p}^{\prime}, \underline{\rho}\right)=e^{i k \rho}\left(\left(\underline{e}_{p} \cdot \underline{e}_{p}^{\prime}-\underline{e}_{p} \cdot \underline{e}_{p} \underline{e}_{p} \cdot \underline{e}_{p}^{\prime}\right) \frac{1}{k \rho}+\right. \\
& \left.\left(\underline{e}_{p} \cdot e_{p}^{1}-3 e_{p} \cdot e_{p} e_{\rho} \cdot e_{p}^{\prime}\right)\left(\frac{i}{k^{2} \rho^{2}}-\frac{1}{k^{3} \rho^{3}}\right)\right) \text {, }
\end{aligned}
$$

$\rho$ is the magnitude of the (real) vector $\underline{\rho}$, and the multiplier $\mathrm{C}$ is

5 Inasmuch as the above $\psi_{0}{ }_{0}\left(\underline{r}_{0}\right)$ is the response of a dipole antenna in a dipole field, we may evaluate it by using the dipole field of example 4 (c), setting $\underline{p}=i_{e} a_{0} e_{p}$ in that expression, and then using the dipole response expression given in example $8(\mathrm{e})$. 


$$
c=-\frac{3 i}{4} \sqrt{\frac{\eta_{0}}{n_{0}^{1}}\left(1-\left|s_{00}\right|^{2}\right)\left(1-\left|s_{o 0}^{1}\right|^{2}\right)} .
$$

Example 2.2-11: Expansion of $\Psi_{0^{\prime}}\left(\underline{r}_{0}\right)$ in a special case.

The expression in (b) of the preceding example contains the (complete, terminating) asymptotic expansion of $\exp (-i k \rho) \Psi_{0}{ }_{0}\left(\underline{r}_{0}\right)$ in powers of $1 / \rho$. If the same quantity were to be expanded in powers of $1 / \mathrm{r}_{0}$, the result would be algebraically much more complicated and the series would not terminate. Nevertheless such an expansion is of direct interest in the work of section 5. We may give a fairly cogent illustration of both the method and the results of that section by means of a simple special case in which $\underline{r}_{0}, \underline{r}$, and $\underline{x}^{\prime}$ are collinear. In fact let us take $\underline{e}_{p}=\underline{e}_{p}^{\prime}=\underline{e}_{x}, \underline{r}_{0}=r_{0} e$, $\underline{r}=a \underline{e}_{z}, \underline{r}^{\prime}=a^{\prime} \underline{e}_{z}$. Then $(b)$ of example 10 yields

$$
e^{-i k r_{0}} \psi_{0^{\prime}}\left(\underline{r}_{0}\right)=C e^{i k \Delta r}\left(\frac{1}{k \rho}+\frac{i}{k^{2} \rho^{2}}-\frac{1}{k^{3} \rho^{3}}\right)
$$

where (to use a bit of notation from section 5), $\Delta r=\left|\underline{r}^{\prime}-\underline{r}\right|=\left|a^{\prime}-a\right|$, and $\rho=r_{0}+\Delta r$. Now, if $r_{0}>\Delta r$, each inverse power of $\rho$ in the above expression may be expanded in powers of $1 / \mathrm{r}_{0}$, and the terms may be rearranged to form a single infinite series in powers of $1 / \mathrm{r}_{\mathrm{C}}$. Thus one may obtain

$$
e^{-i k r} \Psi_{0^{\prime}}\left(\underline{r}_{0}\right)=C e^{i k \Delta r}\left(\frac{1}{k r_{0}}+\left(\frac{i}{k^{2}}-\frac{\Delta r}{k}\right) \frac{1}{r_{0}^{2}}+\cdots\right)
$$

where the series is convergent or divergent according as $\mathrm{r}_{0}$ is greater or less than $\Delta \mathrm{r}$. (The $\Delta \mathrm{r}$ here corresponds exactly to the $(\Delta \mathrm{r})_{\max }$ in the convergence criterion (5.1-15).) Although the result is algebraically more complicated, the important point is that the domain of convergence is determined in a remarkably simple way by the geometry of the composition of the vector $\underline{\underline{p}}=\underline{r}^{\prime}-\underline{r}+\underline{r}_{0}$. This carries over into the general case. 
Examp1e 2.2-12: Analytical test of a two-identical-antenna measurement technigue formula (II-4.2-15). We evaluate

$$
\frac{1}{\left.\right|_{0} T}\left|\int b_{0}^{\prime}(\underline{P}) d \underline{P}\right|
$$

for a pair of elementary electric dipole antennas, oriented transversely $\left(\underline{e}_{p}=\underline{e}_{p}^{\prime}=\underline{e}_{x}\right.$, say), tuned $\left(s_{00}=S_{o 0}^{\prime}=0\right)$, and with $\eta_{0}$ and $\eta_{0}^{\prime}$ chosen equal. Then from the preceding example we find

$$
\begin{aligned}
\frac{1}{a_{0}} b_{0}^{\prime}(\underline{P}) & =-\frac{3 i}{4} f\left(\underline{e}_{x}, \underline{e}_{x}, \underline{P}+\underline{d e}_{z}\right) \\
& =\frac{3}{8 \pi k^{3}} \int\left(k^{2}-k_{x}^{2}\right) \gamma^{-1} e^{i K \cdot \underline{P}+i \gamma d} d \underline{K}
\end{aligned}
$$

Here the scan plane is defined by the fixed value of $d$. The second form permits easy evaluation of the desired quantity by integration with respect to $\underline{P}$ under the integral sign. One obtains

$$
\begin{aligned}
& \frac{1}{\prod_{0} T}\left|\int b_{0}^{\prime}(\underline{P}) d \underline{P}\right|=\left|\frac{3 \cdot 4 \pi^{2}}{8 \pi k^{3}}\left(k^{2}-0\right) k^{-1} e^{i k d}\right| \\
& =\frac{3 \lambda^{2}}{8 \pi} \text {, }
\end{aligned}
$$

where $\lambda=2 \pi / k$. This is the well-known value for the maximum effective area of an clementary electric dipole antenina. 


\section{SOME THEORY OF CONDITIONALLY NON-SCATTERING AND MINIMUM-SCATTERING ANTENNAS}

The concept of "minimum-scattering" antennas was introduced by Dicke [8], p. $317 \mathrm{ff}$. , as was the use of a scattering matrix description of antennas employing a complete set of vector spherical waves for the representation of fields in space. The theory was advanced by Kahn and Kurss [9], who used the same type of scattering representation. Minimum scattering antennas. (MSA) have especially simple and interesting properties, and represent an idealization of properties approximated by a class of physical antennas. (A half-wave dipole antenna made of thin wire is approximately a minimum scattering antenna.) Wasylkiwiskyj and Kahn [10] devote a long paragraph to an assessment of this idealization. In this section we wish to obtain and state in the PWSM framework some of the main properties of minimum scattering antennas.

We begin our discussion with the consideration of an antenna as a "loaded scatterer." An ante:ana with a passive termination at its terminal surface $S_{0}$ is a scattering object whose scattering properties depend to some extent upon the particular reflection coefficient, $\Gamma$, presented by the load at $S_{0}$. We may examine this dependence starting with the general scattering equations for a general antenna,

$$
\begin{aligned}
& b_{o}=S_{o o} a_{0}+\sum_{q} \hat{s}_{o q} \hat{a}_{q}, \\
& \hat{b}_{p}=\hat{s}_{p o} a_{o}+\sum_{q} \hat{S}_{p q} \hat{a}_{q} .
\end{aligned}
$$


Using $a_{0}=\Gamma b_{0}$, we solve the first equation for $a_{0}$ and then eliminate $a_{0}$ from the second equation, obtaining

$$
\hat{b}_{p}=\hat{S}_{p o} \frac{\Gamma}{1-S_{o o} \Gamma} \sum_{q} \hat{s}_{o q} \hat{a}_{q}+\sum_{q} \hat{S}_{p q} \hat{a}_{q}
$$

which can be written

$$
\begin{aligned}
\hat{b}_{p} & =\left(\sum_{q} \frac{\Gamma}{1-S_{o o} \Gamma} \hat{S}_{p o} \hat{S}_{o q}+\hat{S}_{p q}\right) \hat{a}_{q} \\
& \equiv \sum_{q} \hat{S}_{p q}^{\prime} \hat{a}_{q} .
\end{aligned}
$$

Thus the role of $\Gamma$ in producing modifications of the scattering characteristics is exhibited. The modification consists of separable scatiering terms of the form $\hat{\mathrm{S}}_{\text {po }} \hat{\mathrm{S}}_{\mathrm{oq}}$, which produce scattering in the pattern of the transmitting characteristic with amplitudes depending upon the value of the scalar $\hat{\mathrm{S}}_{\text {oq }} \hat{\mathrm{a}}_{\mathrm{q}}$. (N.b.: In the products of the form $\hat{S}_{\text {po }} \hat{S}_{\text {oq }}$ the order of the factors is material; the product defines a (highly degenerate) transformation of the same dimensionality as a general, nonseparable transformation $\hat{\mathrm{S}}_{\mathrm{pq}} \cdot$ ) In many cases the modification of scattering characteristics producible in a loaded scatterer may be limited and not dramatic; we shall see, however, that a minimum scattering antenna can be regarded as an extreme case of a loaded scatterer.

The distinctive defining property for MSA's characterizes a somewhat larger class of antennas, which we call "conditionally non-scattering" (CNS) antennas and define as follows. A CNS antenna is completely non-scattering when terminated with a 
completely reflecting (i.e., lossless) termination having a critical value of phase, $\Gamma=\Gamma_{C}=\exp \left(i \phi_{C}\right)$. For convenience we take $\Gamma_{C}=1$ (a relatively minor restriction) and also $\mathrm{S}_{\mathrm{OO}}=0$ (which represents considerable simplification). The form of the scattering matrix of a CNS antenna may be found as follows: We note that the critical loading requires $a_{0}=b_{0}$ and consider $\hat{a}_{2} \equiv 0, \hat{a}_{1}$ arbitrary. Then $b_{0}=\hat{s}_{01} \hat{a}_{1}$ and the scattering equations become

$$
\begin{aligned}
& \hat{\mathrm{b}}_{1}=\hat{\mathrm{s}}_{10} \hat{\mathrm{s}}_{01} \hat{\mathrm{a}}_{1}+\hat{\mathrm{s}}_{11} \hat{\mathrm{a}}_{1}, \\
& \hat{\mathrm{b}}_{2}=\hat{\mathrm{S}}_{20} \hat{\mathrm{s}}_{01} \hat{\mathrm{a}}_{1}+\hat{\mathrm{s}}_{21} \hat{\mathrm{a}}_{1} .
\end{aligned}
$$

The non-scattering property now requires $\hat{b}_{1}=0$ and $\hat{b}_{2}=\hat{a}_{1}$ for arbitrary $\hat{a}_{1}$. Hence we must have

$$
\hat{\mathrm{S}}_{11}=-\hat{\mathrm{S}}_{10} \hat{\mathrm{S}}_{01}, \quad \hat{\mathrm{S}}_{21}=\hat{1}-\hat{\mathrm{S}}_{20} \hat{\mathrm{S}}_{01} \text {. }
$$

Similarly, by considering $\hat{a}_{1}=0, \hat{a}_{2}$ arbitrary, one finds

$$
\hat{S}_{12}=\hat{1}-\hat{S}_{10} \hat{S}_{02}, \quad \hat{S}_{22}=-\hat{S}_{20} \hat{S}_{02} \text {. }
$$

Hence, the (plane-wave) scattering matrix for a CNS antenna has the remarkably restricted form

$$
\hat{S}=\left(\begin{array}{ccc}
0 & \hat{S}_{01} & \hat{S}_{02} \\
\hat{S}_{10} & -\hat{S}_{10} \hat{S}_{01} & \hat{1}-\hat{S}_{10} \hat{S}_{02} \\
\hat{S}_{20} \cdot \hat{i}-\hat{S}_{20} \hat{S}_{01} & -\hat{S}_{20} \hat{S}_{02}
\end{array}\right) .
$$


Here (and occasionally elsewhere) we use the simple notation $\hat{\mathrm{S}}$ to symbolize the whole antenna scattering-matrix. We can now define a minimum-scattering antenna as a lossless conditionally non-scattering antenna. (What we have just defined would be called by Kahn and Kurss [9] a "one-port, canonical minimumscattering antenna.") We observe that the product terms in (3) represent true scattering, that is, $\hat{t}_{p q}=-\hat{S}_{p o} \hat{S}_{o q}$, and that this property characterizes (3).

Example 3-1: If $\underline{s}_{\mathrm{po}}(\underline{\mathrm{K}})$ and $\underline{\mathrm{s}}_{\mathrm{Oq}}(\underline{\mathrm{L}})$ individually satisfy the transmittingreceiving reciprocity relations $(1-15)$, then $\underline{t}_{\mathrm{pq}}(\underline{K}, \underline{\mathrm{L}})=-\underline{\mathrm{s}}_{\mathrm{po}}(\underline{\mathrm{K}}) \underline{\mathrm{s}}_{\mathrm{Oq}}(\underline{\mathrm{L}})$ will satisfy the scattering reciprocity relation (1-16).

We next find the explicit constraints imposed by the condition of losslessness. For this and some other calculations relating to MS antennas it is an appreciable convenience to have a (temporary) notation more compact than the "hatted" symbols already in use. Wo define the matrices

$$
\begin{aligned}
\left.\alpha=\left(\begin{array}{l}
\hat{a}_{1} \\
\hat{a}_{2}
\end{array}\right), \quad \quad \quad \quad=\left(\begin{array}{l}
\hat{b}_{1} \\
\hat{b}_{2}
\end{array}\right), \quad \begin{array}{l}
\hat{s}_{10} \\
\hat{s}_{20}
\end{array}\right), \quad \text { and } h=\left(\begin{array}{ll}
\hat{S}_{01} & \left.\hat{S}_{02}\right), \\
\sigma & \hat{n}_{r}
\end{array}\right) .
\end{aligned}
$$

and observe that $\sigma$ and $h$ are both Hermitian, they commute with each other, and $\sigma$ is self inverse. That is, we have

$$
\sigma^{*}=\sigma, \quad h *=h, \quad \sigma h=h \sigma, \quad \text { and } \sigma^{2}=\left(\begin{array}{ll}
\hat{i} & 0 \\
0 & \hat{1}
\end{array}\right) \text {. }
$$


The scattering matrix for a MS antenna now appears as

$$
\hat{S}=\left(\begin{array}{ccc}
0 & R \\
T & \sigma & -T R
\end{array}\right)
$$

and the scattering equations read

$$
\begin{aligned}
& \mathrm{b}_{0}=\mathrm{R} \alpha, \\
& \beta=\mathrm{Ta} \mathrm{o}_{0}+(\sigma-\mathrm{TR}) \alpha .
\end{aligned}
$$

Losslessness means, of course, that outgoing power from the antenna must equal incoming power for every state of excitation, including evanescent $\mathrm{PW}$ components in the incident field. Thus the full consideration of losslessness could lead to considerable complication. We can obtain simple necessary conditions for losslessness under the assumption that evanescent components in incident fields are excluded, which permits us to use (II-1.4-9) instead of (II-1.4-8). In the absence of evanescent components in incident fields, losslessness is expressed by

$$
n_{0}\left|b_{0}\right|^{2}+\beta * h \beta=n_{0}\left|a_{0}\right|^{2}+\alpha * h \alpha
$$

(wherein the notation of (II-1.4-10a) is applicable). Eliminating $b_{0}$ and $\beta$ and making use of the arbitrariness of $a_{0}$ and $\alpha$, we find that we must have

$$
\left(\begin{array}{cc}
0 & \mathrm{~T} * \\
\mathrm{R} * & \sigma-\mathrm{R} * \mathrm{~T} *
\end{array}\right)\left(\begin{array}{cc}
n_{0} & 0 \\
0 & h
\end{array}\right)\left(\begin{array}{cc}
0 & \mathrm{R} \\
\mathrm{T} & \sigma-\mathrm{TR}
\end{array}\right)=\left(\begin{array}{cc}
n_{0} & 0 \\
0 & h
\end{array}\right) ;
$$


which in turn implies the scalar equation

$$
T * h T=n_{0}
$$

the row-matrix equation

$$
T * h(\sigma-T R)=0
$$

(as we11 as its Hermitian conjugate), and finally the squarematrix equation

$$
n_{O} R * R+(\sigma-R * T *) h(\sigma-T R)=h .
$$

It is easily shown that (9a) and (9b) together imply (9c); hence we may use (9a) to simplify (9b) to obtain

$$
\mathrm{T} * \mathrm{~h} \sigma=\mathrm{n}_{\mathrm{O}} \mathrm{R}
$$

and state that (9a) and (10a) are necessary and sufficient conditions for the validity of (7).

Equations (9a), (9b), and (9c) constitute a rather esoteric expression of "weighted unitarity" of the partitioned antenna scattering-matrix $\hat{S}$ (cf. example 3.2 below). Equation (9a) represents the squared magnitude of the first column of $\hat{\mathrm{S}}$ and states that radiated power equals input power in the transmitting mode--a fact already expressed in detail for any lossless antenna in (II-1.4-13). Equation (9c) represents the normalization of the second column of $\hat{S}$ and states essentially that power extracted from an incident field is in toto either scattered or received.

\footnotetext{
${ }^{6}$ It is also easily shown that $(9 b)$ and (9c) together imply (9a); but apparently (9a) and $(9 c)$ do not imply (9b)--at any rate the author has not been able to prove the implication.
} 
Equation (9b) states a kind of orthogonality between the two previous (transmitting and receiving) modes of operation and implies a relation between transmitting and receiving characteristics, which holds whether or not the antenna is reciprocal. This last property is clearly shown in a component form of (10a), which reads

$$
\left.n_{m} \overline{S_{20}(m, \underline{K})}=\eta_{0} S_{01}(m, \underline{K}), \quad n_{m} \overline{S_{10}(m, \underline{K}}\right)=n_{0} S_{02}(m, \underline{K}) .
$$

These equations relate receiving and transmitting properties (as do the reciprocity relations) but the properties related pertain specifically to identical values of $\underline{k}$ (rather than to reversed values) and the relations are not established for $K>k$.

Example 3-2: The scattering matrix $\hat{S}$ of a minimum-scattering antenna possesses the (left and right) inverse

$$
\hat{S}^{-1}=\left(\begin{array}{cc}
0 & R \\
\sigma T & \sigma-\sigma T R \sigma
\end{array}\right) .
$$

A further consequence of this property is that $\hat{\mathrm{S}}$ can be made unitary by a suitable power renormalization. (In the context of this monograph, this example is purely an exercise.)

At least in some of the older writings on antenna theory (e.g., Kraus [11], p. 46) one encounters statements to the effect that a (lossless) receiving antenna provided with a matched load scatters as much power ${ }^{7}$ as it delivers to the

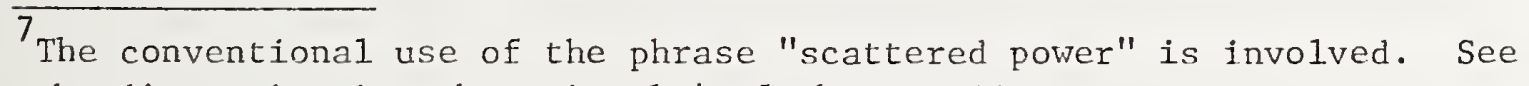
the discussion in subsection 1.4 of Chapter II. 
load. The arguments given do not really establish this result, which is in fact untrue in general, and the inequality may go in either direction. For minimum-scattering antennas the equality does hold, as we proceed to show.

We consider a MS antenna, provided with a reflectionless termination, operating in its receiving (and scattering) mode. Scattered power is, by definition,

$$
\mathrm{P}_{\mathrm{S}}=\frac{1}{2}\left(\beta^{*}-\alpha^{*} \sigma\right) \mathrm{h}(\beta-\sigma \alpha)
$$

successively using (6b), (9a), (6a), and (II-1.1-4), we obtain

$$
\begin{aligned}
P_{S} & =\frac{1}{2} \alpha * R * T * h T R \alpha \\
& =\frac{1}{2} n_{0} \alpha * R * R \alpha \\
& =\frac{1}{2} n_{0}\left|b_{0}\right|^{2} \\
& =P_{r e c},
\end{aligned}
$$

as was to be shown.

The appellation "minimum scattering" is suggested by comparison of the property just derived with the corresponding property of classes of lossless antennas for which it can be shown that $\mathrm{P}_{\mathrm{S}}>\mathrm{P}_{\mathrm{rec}}$. Although this comparative property is intriguing, it is, at least for our purposes, not of direct interest. Consequently we give a very brief discussion and merely quote some results, obtained using the PWSM formulation, pertaining to classes of comparison antennas. Although the steps in the derivations are analogous to the steps in the 
corresponding derivations in Kahn and Kurss [9], the classes of comparison antennas arrived at are not identical to those in that reference. Thus the results are in a sense new. We have also supplemented the previous work with some analytical examples and counter-examples.

We consider lossless antennas, tuned $\left(S_{00}=0\right)$ and provided with matched receivers $\left(\Gamma_{L}=0\right)$--circumstances also pertaining to (11); and we state conditions sufficient to. insure

$$
\mathrm{P}_{\mathrm{S}}>\mathrm{P}_{\mathrm{rec}}
$$

for the considered antennas.

(a) If

$$
\left.\begin{array}{l}
n_{0} s_{01}(m, \underline{K})=n_{m} s_{20}(m, \underline{K}) e^{i c}, \\
n_{0} s_{02}(m, \underline{K})=n_{m} s_{10}(m, K) e^{i c},
\end{array}\right\}
$$

where $c$ is any real constant independent of $\underline{K}$, then (13) will hold for arbitrary incident fields (not containing evanescent PW components).

(b) If the transmitting and receiving characteristics of the considered antenna obey reciprocity (whether or not the scattering characteristics do) and the spectra of the incident fields are constrained by

$$
\underline{A}_{2}(\underline{K})=\overline{A_{1}(-\underline{K})}
$$


then (13) will hold. The incident electromagnetic field corresponding to (15) of course has special properties; in particular, $\underline{E}_{t}$ is real, $\underline{H}_{t}$ is pure imaginary, on the plane $z=0$.

Analytical examples of lossless, non-minimum-scattering antennas show that the indefinite case $\mathrm{P}_{\mathrm{S}}<\mathrm{P}_{\mathrm{rec}}$ may occur. We have not found instances of the negative semidefinite case $\mathrm{P}_{\mathrm{S}}<\mathrm{P}_{\mathrm{rec}}$ !

An interesting theorem results from the combination of the conservation equation (10) with reciprocity. If the MS antenna is reciprocal, then equations $(1-15)$ also apply and we find

$$
\overline{S_{20}(\mathrm{~m}, \underline{K})}=-\mathrm{S}_{10}(\mathrm{~m},-\underline{\mathrm{K}}), \quad \overline{\mathrm{S}_{02}(\mathrm{~m}, \underline{\mathrm{K}})}=-\mathrm{S}_{01}(\mathrm{~m}, \underline{-\underline{K}}) .
$$

An immediate corollary is that the power gains (or effective areas) of a reciprocal MS antenna are equal in diametrically opposite directions. Equation (16) also contributes to the results in examples 5 through 8 .

Example 3-3:

(a) The expression of (10) in terms of $\underline{s}_{q 0}$ and $\underline{s}_{\mathrm{oq}}$ is

$$
\underline{\underline{n}} \cdot \overline{\underline{S}_{20}(\underline{K})}=n_{0} \underline{S}_{01}(\underline{K}), \quad \underline{n} \cdot \overline{\underline{S}_{10}(\underline{K})}=n_{0} \underline{S}_{02}(\underline{K}) .
$$

(b) The expression of (16) in terms of $\underline{s}_{q 0}$ and $\underline{S}_{0 q}$ is

$$
\overline{\underline{S}_{20}(\underline{K})}=\underline{S}_{10}(\underline{-K}), \quad \overline{S_{02}(\underline{K})}=\underline{S}_{01}(\underline{-K}) .
$$

The disappearance of the minus signs between the members of the equations (16) is accounted for by the relation $\underline{k}_{m}(-\underline{K})=-\underline{k}_{m}(\underline{K})$. 
Example 3-4: Expression of (10) and (16) in terms of $\underline{s}_{-q 0}$ and $\underline{s}_{\mathrm{oq}}$.

(a) Equation (II-1.6-15) furnishes

$$
\underline{s}_{02}(\underline{K})=\frac{\gamma}{k} s_{02}(1, \underline{K}) \underline{e}_{\|}\left(\underline{k}^{+}\right)+S_{02}(2, \underline{K}) \underline{e}_{\perp}(\underline{K}) .
$$

Equation (10b) requires

$$
\begin{aligned}
\underline{s}_{02}(\underline{K}) & =\frac{\gamma}{k} \frac{\omega \varepsilon}{n_{0} \gamma} \overline{s_{10}(1, \underline{K})} \underline{e}_{\|}\left(\underline{k}^{+}\right)+\frac{\gamma}{\omega \mu \eta_{0}} \overline{s_{10}(2, \underline{K})} \underline{e}_{\perp}(\underline{K}) \\
& =\frac{\gamma}{n_{0} \omega \mu}\left\{\frac{k}{\gamma} s_{10}(1, \underline{K}) \underline{e}_{\|}\left(\underline{k}^{+}\right)+\overline{s_{10}(2, \underline{K})} \underline{e}_{\perp}(\underline{k})\right) .
\end{aligned}
$$

Hence, using (II-1.6-3) and writing $\omega \mu=k / Y_{0}$, we have

$$
\underline{s}_{02}(\underline{K})=\frac{Y_{0}^{\gamma}}{\eta_{0} k} \overline{s_{10}(\underline{K})}
$$

Similarly, for the other member of (10b) we find

$$
\underline{s}_{01}(\underline{k})=\frac{Y_{0} \gamma}{n_{0} k} \overline{s_{20}(\underline{K})} .
$$

(b) To obtain (16) expressed in terms of $\underline{s}_{q 0}$ and $\underline{s}_{0 q}$ we may apply the reciprocity relation (1-15) to the results just obtained. This yields

$$
\underline{\underline{s}}_{20}(\underline{K})=\underline{s}_{10}(\underline{-K}), \quad \overline{s_{02}(\underline{K})}=\underline{s}_{01}(-\underline{K}) .
$$

We may complete the description of the lossless, reciprocal, elementary, electric and magnetic dipole antennas considered in subsection 2.2 in a natural way by endowing them with the condi- 
tionally non-scattering property (so that they become reciprocal MS antennas). In the next section the properties of these completely described, particularly simple antennas will be used in examples illustrating the complete solvability of classes of coupled antenna problems (including the possibility of relatively easy numerical evaluation). Some of the scattering properties of these special. antennas are brought out in the examples immediately following.

For brevity the classes of antennas currently under discussion will be identified by the codes REDMS and RMDMS, where "R" stands for reciprocal, "ED" and "MD" stand for (elementary) electric and magnetic dipole, and "MS" denotes minimum scattering (as before).

\section{Example 3-5: Phase of REDMS- and RMDMS-antenna normalization constants.}

Combining (16) (or, more conveniently, the result in example $4(\mathrm{~b})$ ) with the expressions for $\mathrm{s}_{\mathrm{qo}}$ given in examples $2.2-8$ and $2.2-9$, one finds that the normalization constants $\mathrm{N}_{\mathrm{e}}=\mathrm{p} /\left(\mathrm{ia}_{\mathrm{o}}\right)$ and $\mathrm{N}_{\mathrm{m}}=\mathrm{m} / \mathrm{a}_{\mathrm{o}}$ must both be real (positive or negative). This result can be attributed to the choice of $\Gamma_{c}=1$ in the definition of CNS antennas; the ambiguity that remains corresponds to an ambiguity of one-half waveguide wavelength in the position of $\mathrm{S}_{\mathrm{o}}$ in the antenna feed waveguide. The results of this example enable us to obtain results free of ambiguity in the next three examples.

Example 3-6: th for REDMS antennas. Referring to (2.2-11) for the definition of $t_{4 p}$ and to examples 4 and 2.2-8 for data, one finds for an antenna of the specified type situated at the point $\underline{r}_{0}$, 


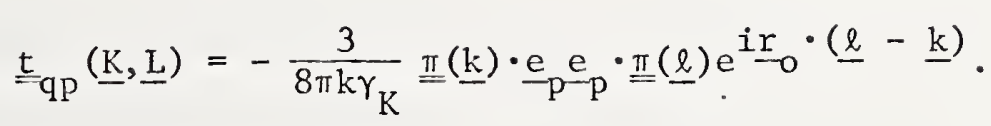

Note that there is no sign or phase indeterminacy in this result.

Example 3-7: th for RMDMS antennas. Referring to $(2.2-11)$ for the definition of $t_{q p}$ and to examples 4 and 2.2-9 for data, one finds for an antenna of the specified type situated at the point $\underline{r}_{0}$,

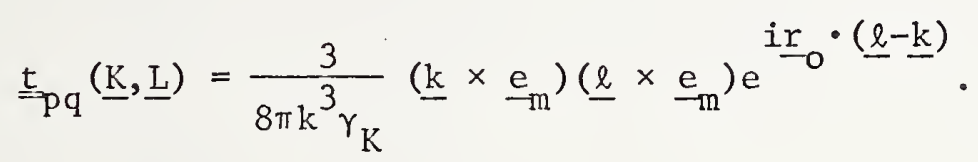

Example 3-8: Polarizabilities of REDMS and RMDMS antennas as loaded scatterers. The intrinsic scattering properties of the antennas being considered may be characterized by electric and magnetic polarizability tensors defined so that

$$
\underline{p}=\underline{x}_{e} \cdot \underline{E}, \quad \underline{m}=\underline{x}_{m} \cdot \underline{H},
$$

respectively, where $\underline{E}$ and $\underline{H}$ belong to the exciting fields. Next, using (3) and (6), we write an equation describing (bona fide) scattering in the case being considered:

$$
\underline{\mathrm{b}}_{\mathrm{qs}}(\underline{\mathrm{K}})=(\Gamma-1) \underline{\mathrm{s}}_{\mathrm{qo}}\left(\underline{K}^{\mathrm{C}} \mathrm{b}_{\mathrm{o}} .\right.
$$

Note that we have enriched the example slightly by including the effect of $\Gamma \neq 0$. The CNS property is already manifest in the factor $(\Gamma-1)$. 
(a) ED case. The expression for $\underline{b}_{q s}$ in terms of $\underline{p}$ is provided in example 2.2-4; expressions for $\underline{s}_{q}$ and for the dipole receiving response $b_{0}$ are found in example 2.2-8. Putting these together one finds

$$
x_{e}=(\Gamma-1) 3 \pi i \varepsilon k^{-3}{ }_{-p}-p
$$

(b) $\mathrm{MD}$ case. The expression for $\underline{\mathrm{b}}_{\mathrm{qs}}$ in terms of $\mathrm{m}$ is provided in example 2.2-5; expressions for $\underline{s}_{q o}$ and for the receiving response $b_{0}$ are found in example 2.2-9. In this case one finds

$$
x_{m}=(\Gamma-1) 3 \pi i k^{-3} \underset{m}{e} e_{m}
$$

4. CLASSES AND EXAMPLES OF SOLVABLE TRANSMISSIONAND REFLECTION - SYSTEM PROBLEMS

The following definitions and remarks are intended to place the work of this section in perspective. At worst, "solvable" means reducible by "quadratures" to a finite-dimensional, 1inear, algebraic (eigenvalue) problem. At best, "solvable" means additionally that the integrals are tractable and that numerical. results may relatively easily be obtained. Further, we are concerning ourselves with complete and exact solutions (in terms of given data); this imples that interactions (multiple reflections) between elements of the systems considered must be fully accounted for. If the role of scattering were to be neglected, solutions would be given essentially by the transmission- and reflectionintegrals, which were introduced in section II- 2 as the first 
approximations provided by Liouville-Neumann series solutions. In the present section we do not use the Liouville-Neumann series approach, but the method used sheds some light on that approach.

The basic equations for the problem of two coupled antennas are (1), (2), and (5) of section 2 of II. The complete solution stems from the solution of the two separate (not simultaneous) linear integral equations

$$
\begin{array}{ll}
\hat{b}_{1}=\hat{S}_{10} a_{0}+\hat{S}_{11} \hat{R}^{\prime} \hat{b}_{1}, & \left(a_{0}^{\prime}=0\right) \\
\hat{b}_{2}^{\prime}=\hat{S}_{20}^{\prime} a_{0}^{\prime}+\hat{S}_{22}^{\prime} \hat{R}_{2}^{\prime} . & \left(a_{0}=0\right)
\end{array}
$$

Here

$$
\begin{aligned}
& \hat{\mathrm{R}}^{\prime} \equiv \hat{\mathrm{T}}_{12} \hat{\mathrm{S}}_{22}^{\prime} \hat{\mathrm{T}}_{21}, \\
& \hat{\mathrm{R}} \equiv \hat{\mathrm{T}}_{21} \hat{\mathrm{S}}_{11} \hat{\mathrm{T}}_{12} .
\end{aligned}
$$

Equation (1a) (which is explicit in section II-2) applies with $a_{0}^{\prime}=0$, while $(2 b)$ applies with $a_{0}=0$. From the solutions of these equations we can calculate the elements of the system 2 -port scattering-matrix (defined in (II-2-10)) and also the spectra of the fields (hence the fields themselves) between the elements of the system and away from the entire system in either direction. We shall content ourselves with calculation of the matrix elements, and we note that reflection-system solutions are given by $M_{0}$ and $M_{O^{\prime} \mathrm{O}^{\prime}}$, since the expressions for these quantities do not involve any distinction between a passive antenna and any other passive scatterer. 
A scattering characteristic of the form 8

$$
\underline{\underline{S}}_{p p}(\underline{K}, \underline{L})=\sum_{i=1}^{n} \underline{v}_{p o}^{j}(\underline{K}) \underline{v}_{o p}^{i}(\underline{L}),
$$

(in which the $\underline{v}_{\mathrm{po}}^{\mathrm{j}}(\underline{K})$ satisfy $\underline{\mathrm{e}}_{\mathrm{K}} \cdot \underline{v}_{\mathrm{po}}^{\mathrm{i}}(\underline{K})=0$ and are linearly independent among themselves, and similarly, the $\underline{v}_{\text {op }}^{i}(\underline{L})$ satisfy $\underline{v}_{\mathrm{vp}}^{i}(\underline{L}) \cdot \underline{e}_{\ell}=0$ and are linearly independent among themselves) will be said to be "degenerate" ([12], p. 114) or of "finite rank" ([13], p. 161). Jf, in particular, $n=1$, the scattering characteristic will be said to be separable. (We have encountered separable scattering in (3-2) and (3-3).) A degenerate scattering characteristic will be represented in our symbolic (shorthand) notation by

$$
\hat{\mathrm{S}}_{\mathrm{pp}}=\sum_{i=1}^{n} \hat{\mathrm{V}}_{\mathrm{po}}^{\mathrm{i}} \hat{\mathrm{V}}_{\mathrm{op}}^{\mathrm{i}}
$$

Next we observe that if either of the scattering operators $\hat{\mathrm{S}}_{11}$ or $\hat{\mathrm{S}}_{22}$ is degenerate, then both of the kernel operators in the integral equations ( $1 \mathrm{a}$ ) and ( $1 \mathrm{~b}$ ) will also be degenerate. This means that both integral equations may be reduced by quadratures to systems of $n$ linear algebraic equations, permitting the application of wel1-known, basic theory given, e.g., in [12] and [13]. Thus degeneracy of one of the scattering operators is a sufficient condition for solvability in the sense defined above.

\footnotetext{
We conld equally well define degenerate scattering in terms of $\underline{S}_{\mathrm{pp}}(\underline{K}, \mathrm{~L})$, using $e_{z}$-transverse vectors to construct the dyadic, and the notation (4) would be equally applicable. For present purposes the choice made in (3) seems more convenient.
} 
Example 4-1: Is an infinite, perfectly-reflecting, plane reflecting surface (represented by $\left.\underline{\underline{S}}_{22}(\underline{K}, \underline{L})=-\left(\underline{\underline{1}}-\underline{e}_{2} \underline{e}_{2}\right) \delta(\underline{K}-\underline{L})\right)$ a degenerate scatterer?

Example 4-2: Consider a scattering operator represented by the dot product of two dyadics, one a function of $k$ only, the other a function of $\stackrel{\ell}{\text { only, }}$

$$
\underline{\underline{A}}(\underline{k}, \underline{l})=\underline{\underline{B}}(\underline{k}) \cdot \underline{\underline{C}}(\underline{l}) .
$$

Show that $\underline{A}$ is a degenerate operator of rank not greater than 3 . Thus, in particular, the dyadic $\underline{\underline{\pi}}(\underline{\mathrm{k}}) \cdot \underline{\underline{\pi}}(\underline{\mathrm{l}})$, which occurs in the scattering characteristic of a small dielectric sphere, is a degenerate operator of rank 3. (The special form of the $\underline{\pi}^{\prime}$ 's does not enable one to write their product as a sum of fewer than three dyads each possessing the separability property, but ic does greatly simplify calculations involving their product.)

We will illustrate the conclusion of the preceding paragraph with a number of examples, considering first and in most detail a system with a general antenna (or scattering object) on the right and a separable-scattering antenna on the left. Even this simple case, in which $\mathrm{n}=1$, is of appreciable interest.

The following equations and definitions are listed for reference: The scattering equations

$$
\begin{aligned}
& b_{0}=s_{00} a_{0}+\hat{s}_{01} \hat{a}_{1}, \\
& b_{0}^{\prime}=S_{00}^{\prime} a_{0}^{\prime}+\hat{S}_{02}^{\prime} \hat{a}_{2}^{\prime}
\end{aligned}
$$


for the antenna on the left and on the right respectively; the joinjing equations

$$
\hat{a}_{1}=\hat{\mathrm{T}}_{12} \hat{\mathrm{b}}_{2}^{\prime}, \quad \hat{\mathrm{a}}_{2}^{\prime}=\hat{\mathrm{T}}_{21} \hat{\mathrm{b}}_{1}
$$

and the transmission- and reflection-integral definitions

$$
\begin{array}{ll}
\Phi_{00}=\left(\hat{S}_{01} \hat{\mathrm{R}}^{\prime} \hat{\mathrm{S}}_{10}\right), & \Psi_{00^{\prime}}=\left(\hat{\mathrm{S}}_{01} \hat{\mathrm{T}}_{12} \hat{\mathrm{S}}_{20}^{\prime}\right), \\
\Psi_{0^{\prime} 0}=\left(\hat{\mathrm{S}}_{02}^{\prime} \hat{\mathrm{T}}_{21} \hat{\mathrm{S}}_{10}\right), & \Phi_{0^{\prime} 0^{\prime}}=\left(\hat{\mathrm{S}}_{02}^{\prime} \hat{\mathrm{R}}_{20}\right) .
\end{array}
$$

Equations (5) and (6) are reproduced from section II-2; the set of definitions is a slight extension of those already used there. Here and in what follows we use parentheses to set off scalar products.

We first consider the half of the problem with $a_{0}^{\prime}=0$. With the separable scattering property incorporated, the integral equation (1a) becomes

$$
\hat{\mathrm{b}}_{1}=\hat{\mathrm{S}}_{100_{0}}+\hat{\mathrm{V}}_{10}\left(\hat{\mathrm{V}}_{01} \hat{\mathrm{R}}^{\prime} \hat{\mathrm{b}}_{1}\right)
$$

Premultiplication by $\hat{V}_{01} \hat{R}^{\prime}$ yields an equation immediately solvable for the scalar $x=\left(\hat{V}_{01} \hat{R}^{\prime} \hat{b}_{1}\right)$,

$$
x=\left(\hat{V}_{01} \hat{R}^{\prime} \hat{S}_{10}\right) a_{0}+\left(\hat{V}_{01} \hat{R}^{\prime} \hat{V}_{10}\right) x
$$


Hence

$$
\hat{b}_{1}=\hat{S}_{100_{0}}+\hat{v}_{10} \frac{\left(\hat{V}_{01} \hat{R}^{\prime} \hat{S}_{10}\right)}{1-\left(\hat{V}_{01} \hat{R}^{\prime} \hat{V}_{10}\right)} a_{0} .
$$

The result affords us an opportunity to comment on some relationships to theory, which have been suppressed in the present work. Equation (9) is also obtainable from the Liouville-Neumann series (II-2-9), which, for $\left|\left(\hat{V}_{01} \hat{R}^{\prime} \hat{V}_{10}\right)\right|<1$, is valid and summable as a geometric series to yield (9). Equation (9) itself thus furnishes an anaiytic continuation of the series solution, valid provided merely $\left(\hat{V}_{01} \hat{R}^{\prime} \hat{V}_{10}\right) \neq 1$. Further to the theory of integral equations in this simple case, it may be noted that $\hat{S}_{10}$ is the single eigenvector, and $\left(\hat{V}_{01} \hat{R}^{\prime} \hat{V}_{10}\right)$ the corresponding eigenvalue, of the eigenvalue problem $\hat{S}_{11} \hat{R}^{\prime} \hat{\psi}=\Lambda \hat{\Psi}$.

We return to the calculation at hand. Recalling the definitions of $M_{00}$ and $M_{O^{\prime}}{ }$, we use (5a), (2a), (9), and (7) to obtain

$$
M_{00}=S_{00}+\Phi_{00}+\frac{\left(\hat{S}_{01} \hat{R}^{\prime} \hat{V}_{10}\right)\left(\hat{V}_{01} \hat{R}^{\prime} \hat{S}_{10}\right)}{1-\left(\hat{V}_{01} \hat{R}^{\prime} \hat{V}_{10}\right)}
$$

similarly, using (5b), (6), (9), and (7), we obtain

$$
M_{O^{\prime} O}=\Psi_{O^{\prime} O}+\frac{\left(\hat{\mathrm{S}}_{02}^{\prime} \hat{\mathrm{T}}_{21} \hat{\mathrm{V}}_{10}\right)\left(\hat{\mathrm{V}}_{01} \hat{\mathrm{R}}^{\prime} \hat{\mathrm{S}}_{10}\right)}{1-\left(\hat{\mathrm{V}}_{01} \hat{\mathrm{R}}^{\prime} \hat{V}_{10}\right)} .
$$

To complete the solution of the problem posed, we set $a_{0}=0$ and derive expressions for $M_{O_{0}}$, and $M_{O^{\prime} O^{\prime}}$. The integral equation (1b) applies and reads 


$$
\hat{b}_{2}^{\prime}=\hat{S}_{20}^{\prime} a_{0}^{\prime}+\hat{S}_{22}^{\prime} \hat{T}_{21} \hat{V}_{10}\left(\hat{V}_{01} \hat{T}_{12} \hat{b}_{2}^{\prime}\right)
$$

We premultiply by $\hat{\mathrm{V}}_{01} \hat{\mathrm{T}}_{12}$ and obtain for the scalar $\mathrm{x}=\left(\hat{\mathrm{V}}_{01} \hat{\mathrm{T}}_{12} \hat{\mathrm{b}}_{2}^{\prime}\right)$ the equation

$$
x=\left(\hat{V}_{01} \hat{T}_{12} \hat{S}_{20}^{\prime}\right) a_{0}^{\prime}+\left(\hat{V}_{01} \hat{R}^{\prime} \hat{V}_{10}\right) x
$$

Hence

$$
\hat{b}_{2}^{\prime}=\hat{S}_{20}^{\prime} a_{0}^{\prime}+\hat{S}_{22}^{1} \hat{T}_{21} \hat{V}_{10} \frac{\left(\hat{V}_{01} \hat{T}_{12} \hat{S}_{20}^{\prime}\right)}{1-\left(\hat{V}_{01} \hat{R}^{\prime} \hat{V}_{10}\right)} a_{0} .
$$

(Remarks similar to those following (9) are applicable here; in the present instance, $\hat{\mathrm{S}}_{22}^{\prime} \hat{\mathrm{T}}_{21} \hat{\mathrm{V}}_{10}$ is the eigenfunction and $\left(\hat{\mathrm{V}}_{01} \hat{\mathrm{R}}^{\cdot} \hat{\mathrm{V}}_{10}\right)$ is the eigenvalue pertaining to the associated eigenvalue problem.) Much as in the preceding paragraph, we now use (13), (5a), (6) and (7) to obtain

$$
\mathrm{M}_{00^{\prime}}=\Psi_{00^{\prime}}+\left(\hat{\mathrm{S}}_{01} \hat{\mathrm{R}}^{\prime} \hat{\mathrm{V}}_{10}\right) \frac{\left(\hat{\mathrm{V}}_{01} \hat{\mathrm{T}}_{12} \hat{\mathrm{S}}_{20}^{\prime}\right)}{1-\left(\hat{\mathrm{V}}_{01} \hat{\mathrm{R}}^{\prime} \hat{\mathrm{V}}_{10}\right)}
$$

and we use (13), (5b), (2b), and (7) to obtain

$$
M_{0}^{\prime} 0^{\prime}=S_{00}^{1}+\left[\left(\hat{S}_{02}^{1} \hat{T}_{21} \hat{V}_{10}\right)\left(\hat{V}_{01} \hat{T}_{12} \hat{S}_{20}^{1}\right)\right] \frac{1}{1-\left(\hat{V}_{01} \hat{R}^{\prime} \hat{V}_{10}\right)} \text {. }
$$

Here the quantity in brackets is the reflection integral $\Phi_{0} 0^{\prime}$ for the problem, appearing in factorized form because of the separable scattering property of the antenna on the left. 
Thus, in (10), (11), (14) and (15), we have obtained solutions for the 2 -port matrix elements in terms of quadratures, as promised.

The number of distinct integrals involved in the above results is appreciably reduced if we take the separable scattering antenna to be a conditionally non-scattering antenna, as defined in section 3. This requires $\hat{\mathrm{V}}_{10} \hat{\mathrm{V}}_{01}=-\hat{\mathrm{S}}_{10} \hat{\mathrm{S}}_{01}$ and $\mathrm{S}_{00}=0$ and the specialization of the above expressions leads to

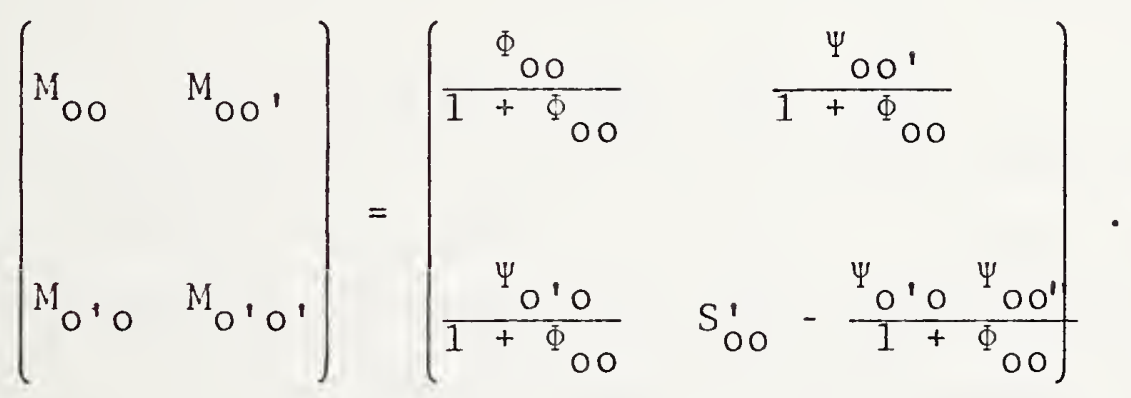

Note that the reflection integral $\Phi_{0} \mathrm{O}^{\prime}$ now appears as the (negative) product of the two transmission integrals involved.

If both antennas are CNS, then $S_{O_{0}}^{\prime}=0$ and $\Phi_{0 O}=\Phi_{O^{\prime} O^{\prime}}=$ - $\Psi_{0}{ }^{\prime} \Psi_{00}$, and the 2 -port scattering matrix may be written

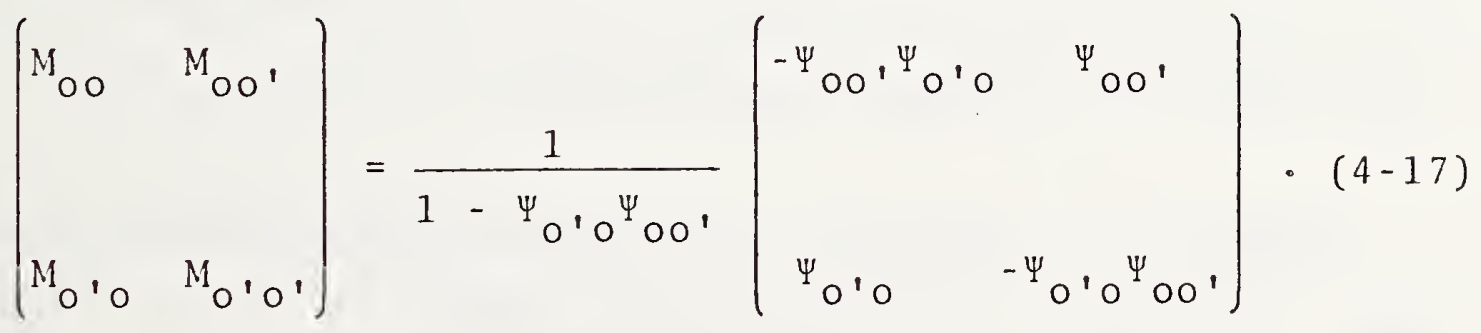


Example 4-3: Show that the system 2-port impedance matrix corresponding to $(17)$ is

$$
\left(\begin{array}{ll}
z_{00} & z_{00^{\prime}} \\
z_{0}^{\prime} 0 & z_{0^{\prime} 0^{\prime}}
\end{array}\right)=\left(\begin{array}{cc}
1 / \eta_{0} & 2 \Psi_{00^{\prime}} / \eta_{0}^{\prime} \\
2 \Psi_{0}{ }_{0} / \eta_{0} & 1 / \eta_{0}^{\prime}
\end{array}\right)
$$

The simplicity of this result is attributable to the CNS property itself and to the specific choices $\Gamma_{C}=1$ and $S_{o O}=0$ made in the original definition. An appearance of even greater simplicity is obtained if one chooses both characteristic admittances equal to unity, $n_{0}=n_{0}^{\prime}=1$.

We still have invoked neither losslessness nor reciprocity. Insofar as losslessness is not required, the result (17) (or equivalently, the result in example 3) is somewhat more general than that of Wasylkiwskyj and Kahn [10], which was obtained for pairs of minimum-scattering antennas. The most nearly comparable equation in [10] appears to be (69).

Example 4-4: Convergence of the Liouville-Neumann series in a special case. We consider a transmission system consisting of a pair of REDMS $^{9}$ antennas, oriented parallel and transverse $\left(\underline{e}_{p}=\underline{e}_{p}^{\prime}=e_{x}\right.$, say), on-axis with separation distance $d$, tuned $\left(S_{c o}=S_{0 O}^{\prime}=0\right)$, and with $n_{0}=n_{0}^{\prime}$. According to the remarks We revert to the abbreviations introduced on page 234. 
under (9), convergence is secured if and only if. the magnitude of the eigenvalue $\mathrm{X}=\left(\hat{\mathrm{V}}_{01} \hat{\mathrm{R}}^{\prime} \hat{\mathrm{V}}_{10}\right)$ is less than unity. For any pair of minimum-scattering antennas, we have for the eigenvalue

$$
x=-\left(\hat{\mathrm{S}}_{01} \hat{\mathrm{T}}_{12} \hat{\mathrm{S}}_{20}^{\prime}\right)\left(\hat{\mathrm{S}}_{02}^{\prime} \hat{\mathrm{T}}_{21} \hat{\mathrm{S}}_{10}\right)=-\Psi_{00^{\prime}} \psi_{0^{\prime}} \mathrm{o}_{0}
$$

For the pair of antennas considered, we have from example 2.2-10

$$
\Psi_{O O^{\prime}}=\Psi_{O^{\prime}}=\frac{3}{4} e^{i \theta}\left(\frac{1}{\theta^{2}}+i\left(\frac{1}{\theta^{3}}-\frac{1}{\theta}\right)\right)
$$

where $\theta=k d$. Setting $|x|=1$, we obtain a cubic equation in $1 / \theta^{2}$; there is only one real root, and the corresponding value of $\mathrm{kd}$ is $(\mathrm{kd})_{\mathrm{c}}=0.87993310 \ldots$. Moreover, it can be shown that $|\mathrm{x}|$ is greater or less than unity according as $\mathrm{kd}$ is less than or greater than $(\mathrm{kd}){ }_{\mathrm{c}}$. This is the origin of the result quoted in section II-2.

Example 4-5: Moo for RCNSA + REDMSA.

(a) Lema--Evaluate the reflection integral $\Phi_{\text {oo }}$ for the system with a reciprocal (otherwise general) antenna on the left, REDMSA on the right, obtaining $\underline{\underline{s}}_{22}^{\prime}$ from example $3-6$.

$$
\Phi_{o 0}=-\frac{3 \pi Y_{0}}{2 k^{2} n_{0}}\left[\underline{e}_{p} \cdot \underline{E}_{0}(\underline{r})\right]^{2} .
$$


Here $E_{0}(\underline{r})$ is the free-space field that would be radiated with $a_{0}=1$. This is basically a well-known type of result that has received consideration for measurement applications $[14,15,15,17]$.

(b) If the antenna on the left is RCNS, then (16) applies and yields

$$
M_{O O}=\frac{\Phi_{O O}(\underline{r})}{1+\Phi_{0 O}(\underline{r})} .
$$

This represents a case of complete solvability if the transmitting and receiving characteristics of the antenna on the left are known. If these characteristics are not known, the above expression still represents a measurement equation in that it can be solved for the component of $\underline{E}$ involved in terms of the observable $M_{00}$ (with only a sign ambiguity). This is one instance, albeit restricted, where theory is adequate to permit measurements to be made in the presence of arbitrarily strong multiple reflections.

Example 4-6: Reciprocal CNS autenna + small dielectric sphere.

(a) Lemma--Evaluate the reflection integral $\Phi_{\text {oo }}$ for a system with a reciprocal antenna on the left, the small dielectric sphere on the right, obtaining $\underline{\underline{s}}_{22}^{\prime}$ from example 2.2-7.

$$
\Phi_{0 \circ}(\underline{r})=2 \pi i a^{3} \frac{\varepsilon_{1}-\varepsilon_{0}}{\varepsilon_{1}+2 \varepsilon_{0}} \frac{Y_{0}}{\eta_{0}}\left[\underline{E}_{0}(\underline{r})\right]^{2} .
$$


Here, as in example 5, $\underline{E}_{0}(\underline{r})$ is the free-space field that would be radiated with $a_{0}=1$. Although the small dielectric sphere does not possess the separable scattering property (see example 2), $\Phi_{00}$ is easily calculated (indeed by steps very similar to those leading to $\Phi_{00}$ in the preceding example)

(b) If we now invoke the CNS property for the antenna on the left, we may apply (16) to obtain

$$
M_{00}=\frac{\Phi_{00}(\underline{r})}{1+\Phi_{00}(\underline{r})} .
$$

For complete solvability it is sufficient that either $\underline{s}_{10}$ or $\underline{E}_{0}$ be known, although the integration to determine the latter from the former would not necessarily be easily done. If we specialize even further and take the reciprocal CNS antenna to be a reciprocal elementary electric (or magnetic) dipole MS antenna, the results of the relatively easy integrations are at hand. Even in these simple cases $M_{00}$ is quite a complicated function of $\underline{r}$. 


\section{LAURENT SERIES ASSOCIATED WITH TRANSMISSION- AND REFLECTION-INTEGRALS}

5.1 Expansion of $\exp \left(-i k r_{0}\right) \Psi_{0}{ }^{\circ}\left(\underline{r}_{0}\right)$ in Powers of $1 / r_{0}$

In this subsection we consider the expansion of the transmission integral

$$
\Psi_{0^{\prime} \mathrm{O}}\left(\underline{\mathrm{r}}_{\mathrm{O}}\right)=\int \underline{\mathrm{s}}_{0}^{\prime}(\underline{\mathrm{K}}) \cdot \underline{\mathrm{s}}_{10}(\underline{\mathrm{K}}) \mathrm{e}^{i \underline{\mathrm{k}} \cdot \underline{\mathrm{r}}_{\mathrm{O}}} \mathrm{d} \underline{\mathrm{K}}
$$

in the form

$$
\Psi_{0^{\prime} \mathrm{O}}\left(\underline{\mathrm{r}}_{0}\right)=\frac{\mathrm{e}^{i k r_{0}}}{\mathrm{r}_{\mathrm{O}}}\left(\mathrm{C}_{10}+\mathrm{C}_{11} \mathrm{r}_{0}^{-1}+\mathrm{C}_{12} \mathrm{r}_{0}^{-2}+\ldots\right) \cdot(5.1-2)
$$

This an expansion of asymptotic type, ${ }^{10}$ but convergence is assured for sufficiently large $r_{0}$ under mild and physically realistic constraints. Under these constraints, the series is the Laurent expansion of $\Psi_{O^{\prime}}\left(\underline{r}_{0}\right) / \exp \left(i k r_{0}\right)$ and is not merely asymptotic. Additionally the theory of this subsection yields for the leading coefficient in (2)

$$
C_{10}=-2 \pi i \gamma\left(K_{0}\right) \underline{s}_{0}^{\prime}\left(\underline{K}_{0}\right) \cdot \underline{s}_{10}\left(\underline{K}_{0}\right),
$$

where $\underline{k}_{0}$ is the transverse part of $\underline{k}_{0}$, and $\underline{k}_{0}=k_{r_{0}} / r_{0}$. This relation is, of course, the main reason for our interest in the expansion (2); the on-axis value of $C_{10}$ is denoted by $A_{00}$ in subsection 3.2 of Chapter II.

$\overline{10} \mathrm{It}$ is well known that a given series of asymptotic type asymptotically represents a class of functions, not a unique function. See, e.g., Erdélyi [24], p. 14. This lack of uniqueness is present whether or not the asymptotic series happens to converge and may be regarded as a defect of representations of asymptotic type. 
In appendix $B$ we derive, as closely related corollaries of (2), (a) the relation between asymptotic electric field and its spectrum and (b) an expansion theorem for the electric field of bounded scatterers. (The asymptotic relation in question is stated in (II-1.2-16) and it has been applied a good many times in this monograph.) These results are not new, but the method of derivation contains new elements. The results are obtained essentially by considering a system consisting of one ideal antenna (elementary electric dipole) and one general antenna or scatterer. Appendix B can be considered as an exercise illustrating some of the significance of the transmission integral and strengthening the theory of (II-1.2-16).

The expansion (2) evaluated with $\underline{r}_{0}=z_{0}-_{z}$, together with similar expansions of the reflection integral and higher iterated integrals in the relevant Liouville-Neumann series, finds application in the extrapolation technique of antenna measurement (Wacker [18], Newell et al. [19]. A brief description of the extrapolation technique is contained in Newell and Kerns [20].)

The work in this subsection is based on some of the work origina11y given in an NBS Report [2] and related to the expansion of the on-axis transmission integral $\Psi_{0}{ }_{0}\left(z_{0} e_{z}\right)$; the method used in the report extends easily to the consideration of the more general transmission integral $\Psi_{O^{\prime} \mathrm{O}}\left(\underline{r}_{\mathrm{O}}\right)$, and this extension is made here. Essentially identical results have been obtained for the on-axis transmission integral by Yaghjian (private communication) using a different method and by Wacker [18] using still another method. Wacker included consideration of lossy transmission media, we do not. 
Our approach to the theory of (2) is motivated by observation of the character of the explicitly evaluated transmission integral in the basic special case of coupled elementary electric-dipole antennas. The transmission integral in question is shown in example 2.2-10 and the critical feature is noted and illustrated in example 2.2-11. This approach easily yields a simple prescription for the value of distance $c_{0}$ such that convergence of (2) is assured for $r_{0}>c_{0}$. (The total effort naturally depends upon the level of rigor sought.) The approach is also applicable without difficultly (except for details of calculation) to obtain expansions in powers of $1 / r_{0}$ and convergence criteria for the reflection integral and for higher integrated integrals in the Liouville-Neumann series. This is illustrated in the reflection integral case in the next subsection.

In all cases the principal hypothesis is that each of the two antennas of a. system be of finite size (each enclosable in a sphere of finite radius). The critical distance, $c_{o}$, is found to be a geometrical constant depending upon the positions of the antennas relative to their respective fiducial coordinate systems as well as upon the size and shape of each antenna. Similar statements apply for systems consisting of one antenna and a scatterer. Although we explicitly consider only the expansions of the transmission and the reflection integrals in this monograph, we may report that the convergence criterion is the same for all iterated integrals pertaining to a given system.

To begin our analysis, we wish to express the coupling product appearing in the integrand of (1) in terms of the three-dimensional, 
spatial, Fourier transforms of equivalent current-densities derived in section 2. In setting up the problem we follow the general procedure established earlier in section II-2.

Equations (2.2-7a, -7b) apply in the case of the transmitting antenna and provide us with

$$
\begin{aligned}
& \underline{U}_{1}(\underline{\mathrm{K}})=\mathrm{C}_{1} \int_{V} \underline{J}_{\mathrm{e}}(\underline{\mathrm{r}}) \mathrm{e}^{-i \underline{\mathrm{k}} \cdot \underline{\mathrm{r}} \mathrm{d} \underline{\mathrm{r}},} \\
& \underline{\mathrm{s}}_{10}(\underline{\mathrm{K}})=\mathrm{\gamma}^{-1} \underline{\mathrm{k}} \times\left[\underline{\mathrm{k}} \times \underline{\mathrm{U}}_{1}(\underline{\mathrm{K}})\right] .
\end{aligned}
$$

Here $C_{1}=1 /(4 \pi \varepsilon \omega), \underline{r}$ is measured from the origin 0 of Oxyz, and the distribution of current density $\underline{J}_{e}$ is by hypothesis confined to a finite region $V$ (fig. 2(a)). This current distribution is characteristic of the isolated transmitting antenna and is considered to be properly normalized with respect to $a_{0}$.

We obtain the receiving characteristic $\underline{s}_{0}^{\prime}$ of the receiving antenna from the appropriate transmitting characteristic of the antenna adjoint to the receiving antenna, using the adjoint reciprocity relation (1-15). Equations (2.2-8a,-8b) are applicable and thus read

$$
\begin{aligned}
& \underline{U}_{2}^{\prime a}(-\underline{K})=C_{1} \int_{V^{\prime}} \underline{J}_{-}^{\prime a}\left(\underline{r}^{\prime}\right) e^{i \underline{k} \cdot \underline{r}^{\prime} d \underline{r}^{\prime},} \\
& \underline{s}_{0}^{\prime}(\underline{K})=\frac{Y_{0}}{n_{0}^{\prime} k} \underline{k} \times\left[\underline{k} \times \underline{U}_{2}^{\prime a}(-\underline{K})\right] .
\end{aligned}
$$


Here $\underline{r}^{\prime}$ is measured from the origin $0^{\prime}$ of the coordinate system $0^{\prime} x^{\prime} y^{\prime} z^{\prime}, O^{\prime}$ is at the point represented by $\underline{r}_{0}$ in the system oxyz, and $\underline{J}_{e}^{a}$ is the normalized current-density distribution characteristic of the adjoint antenna in its transmitting mode. Again, $\underline{J}_{e}^{a}$ is to be non-zero only in a finite region $V^{\prime}$.

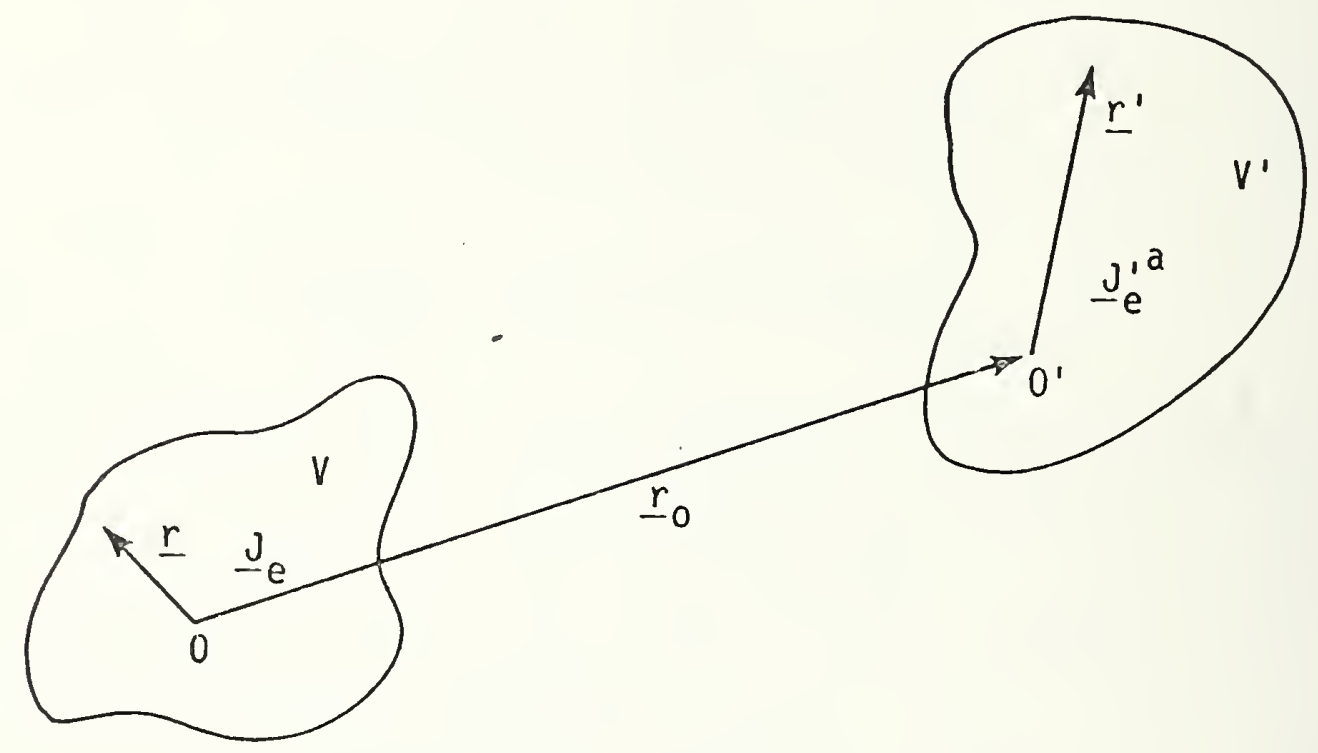

(a)

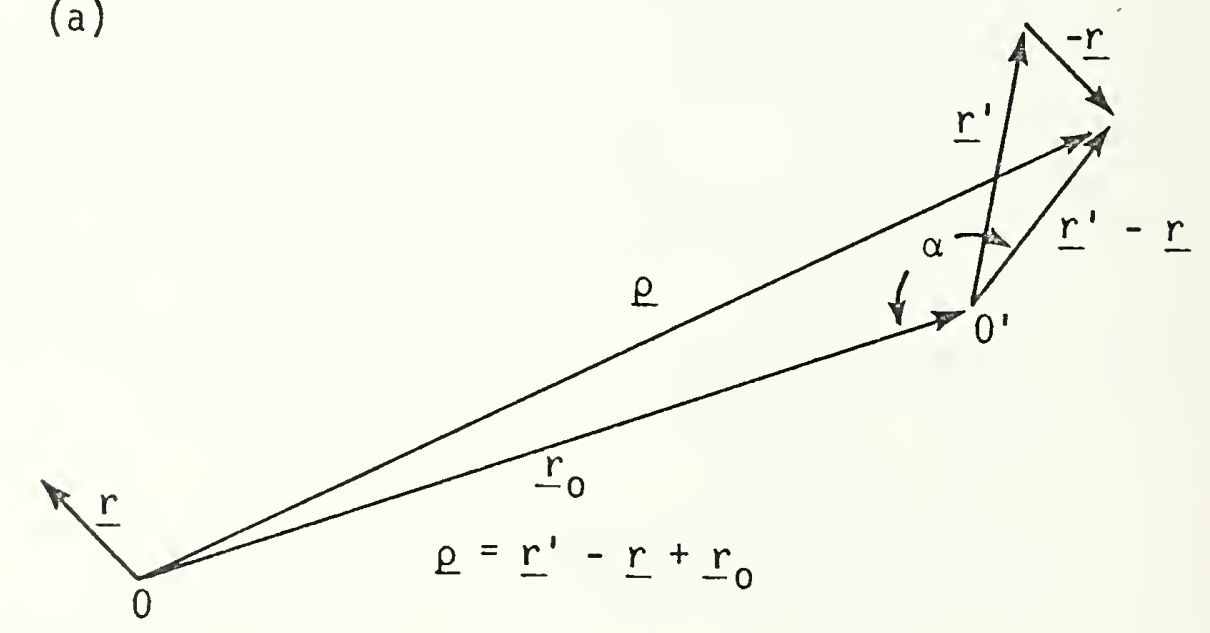

(b)

Figure 2. (a) Domains of $\underline{J}_{e}(\underline{r})$ and $\underline{J}_{e}^{\prime a}\left(\underline{r}^{\prime}\right)$; (b) composition of the vector $\underline{\rho}$. 
After a bit of vector algebra we obtain from (4b) and (5b) the intermediate result

$$
\underline{s}_{0}^{\prime}(\underline{K}) \cdot \underline{s}_{10}(\underline{K})=\frac{Y_{0} k}{n_{0} \gamma} \underline{U}_{1}(\underline{K}) \cdot\left(k^{2} \underline{\underline{1}}-\underline{k k}\right) \cdot \underline{U}_{2}^{\prime a}(-\underline{K})
$$

It will prove convenient to write

$$
\underline{J}_{e}(\underline{r})=\left|\underline{J}_{e}(\underline{r})\right| \underline{e}_{p}(\underline{r}), \quad \underline{J}_{e}^{\prime a}\left(\underline{r}^{\prime}\right)=\left|\underline{J}_{e}^{\prime a}\left(\underline{r}^{\prime}\right)\right| \underline{e}_{p}^{\prime}\left(\underline{r}^{\prime}\right),
$$

thereby defining the complex vectors $\underline{e}_{-}$and $\underline{e}_{-}^{\prime}$ having unit Hermitian magnitude $\left(\underline{e}_{p} \cdot{ }_{-}^{\bar{e}} p=\underline{e}_{p}^{\prime} \cdot \underline{e}_{p}^{\prime}=1\right)$. These unit vectors are, of course, undefined--and unneeded--wherever the associated $\underline{J}_{\mathrm{e}}$ happens to vanish. In much of what follows the arguments $\underline{r}$ and $\underline{r}^{\prime}$ of the quantities in (7) will not be shown explicitly, merely to simplify the typography. We now assemble $(4 a),(5 a),(6)$, and (7), and arrange the integrations to obtain

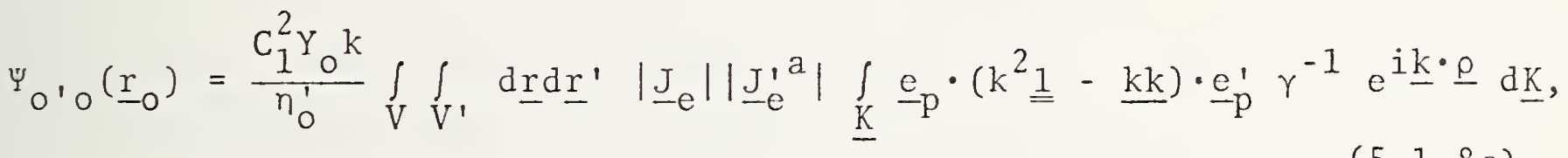
$(5.1-8 a)$

where $\underline{\rho} \equiv \underline{r}_{0}+\underline{r}^{\prime}-\underline{r}$ (Seefig. 2(b)). Let $z_{\text {min }}^{\prime}$ denote the minimum value of $z^{\prime}$ for $\underline{r}^{\prime}$ in $V^{\prime}$, and let $z_{\max }$ denote the maximum value of $z$ for $\underline{r}$ in $V$. Then, to avoid encroachment and to secure convergence of the $\underline{K}$-integral, the inequality $z_{0}+z_{\min }^{\prime}-z_{\max }>0$ must be in force. Now, the K-integral converges uniformly with respect to $\underline{r}$ and $\underline{r}^{\prime}$ (when it converges), and the $\underline{r}$-and $\underline{r}^{\prime}$-integrals are taken over finite domains. Hence we assume the ordering of the integrations in (8a) to be justifiable. Next we notice that the 
result of the $\underline{K}$-integration can be obtained by comparison with the transmission integral for a pair of coupled elementary electric dipole antennas; to some extent, this also provides an interpretation of the whole expression (8a). In fact we obtain

$$
\Psi_{O^{\prime} \mathrm{O}}\left(\underline{r}_{\mathrm{O}}\right)=\mathrm{C} \int_{V} \int_{V^{\prime}} \mathrm{d} \underline{\mathrm{r}}^{\mathrm{d}} \underline{\underline{r}}^{\prime}\left|\underline{J}_{\mathrm{e}}\right|\left|\underline{J}_{\mathrm{e}}{ }^{\mathrm{a}}\right| \mathrm{f}\left(\underline{\mathrm{e}}_{\mathrm{p}}, \underline{\mathrm{e}}_{\mathrm{p}}^{\prime}, \underline{\rho}\right) .
$$

Here $\mathrm{C}$ has absorbed an additional factor $-2 \pi i \mathrm{k}^{3}$, and, as in example 2.2-10,

$$
\begin{aligned}
f\left(\underline{e}_{p}, \underline{e}_{p}^{\prime}, \underline{\rho}\right)=e^{i k \rho}\left(\left(\underline{e}_{p} \cdot \underline{e}_{p}^{\prime}-\underline{e}_{p} \cdot \underline{e}_{\rho}-{ }_{\rho} \cdot \underline{e}_{p}^{\prime}\right) \frac{1}{k \rho}+\right. \\
\left.\left.\left(\underline{e}_{p} \cdot \underline{e}_{p}^{\prime}-3 \underline{e}_{p} \cdot \underline{e}_{\rho} \underline{e}_{\rho} \cdot \underline{e}_{p}^{\prime}\right)\left(\frac{i}{k^{2} \rho^{2}}-\frac{1}{k^{3} \rho^{3}}\right)\right) .9 a\right)
\end{aligned}
$$

In this expression, $\rho$ is the positive square root

$$
\sqrt{r_{0}^{2}-2 r_{0} \Delta r \cos \alpha+(\Delta r)^{2}}
$$

where $\Delta r \equiv\left|\underline{r}^{\prime}-\underline{r}\right|, \alpha$ is the supplement of the angle between $\underline{r}_{0}$ and $\underline{r}^{\prime}-\underline{r}$, and $\underline{e}_{\rho}$ is the "analytic unit vector" defined as $\underline{\rho} / \rho$. (In a moment we shall introduce complex $\underline{\rho}$; when $\underline{\rho}$ is complex, the scalar $\rho$ becomes complex, and the Hermitian magnitude of the "unit" vector $\underline{e}_{p}$ is in general greater than unity.)

It can be shown that the expression (8b) for $\Psi_{O^{\prime}}\left(\underline{r}_{0}\right)$ remains valid for any direction and magnitude of $\underline{r}_{0}$, provided merely that the condition (15) (given below) holds. That is, it is actually necessary only that the domains $V$ and $V^{\prime}$ be disjoint; the "no encroachment" restriction can be circumvented. 
The analytical properties of (9) are decisive for the behavior of $\Psi_{O^{\prime}{ }^{\circ}}\left(\underline{r}_{0}\right)$ itself. In order to state and exploit these properties, we temporarily replace $\underline{r}_{0}$ by the cophasal complex vector

$$
\underline{\zeta}=\underline{r}_{0} e^{i \theta}
$$

where $\theta$ is real and confined to a $2 \pi$ interval, $0<\theta<2 \pi$, say. Then

$$
\begin{aligned}
& |\underline{\zeta}| \equiv \sqrt{\underline{\underline{\zeta} \cdot \bar{\zeta}}}=r_{0}, \\
& \zeta \equiv \sqrt{\underline{\zeta} \cdot \underline{\zeta}}=r_{0} e^{i \theta},
\end{aligned}
$$

and we can also write

$$
\underline{\zeta}=\zeta \underline{\mathrm{e}}_{\mathrm{r}_{0}} .
$$

(a) We now have

$$
\underline{\rho}=\underline{\zeta}+\Delta \underline{r},
$$

and $\rho$ becomes complex in general,

$$
\rho=\sqrt{\underline{\rho} \cdot \underline{\rho}}=\sqrt{\zeta^{2}-2 \zeta \Delta r \cos \alpha+(\Delta r)^{2}} \text {. }
$$


The only singularities of this function of $\zeta$ are the points where it vanishes; that is, the branch points $\zeta=\Delta r \exp ( \pm i \alpha)$. The chosen branch is (one-valued and) analytic in the complex plane for $|\zeta|>\Delta \mathrm{r}$.

(b) Upon examination, we find that the quantity $\underline{e}_{p} \cdot \underline{e}_{p} \underline{e}_{\rho} \cdot \underline{e}_{p}^{\prime}$ appearing in (9) can be written as a rational fractional function of $\zeta$ :

$$
\underline{e}_{p} \cdot \underline{e}_{\rho} \underline{e}_{\rho} \cdot \underline{e}_{p}^{\prime}=\frac{\underline{e}_{p} \cdot\left(\zeta \underline{e}_{r_{0}}+\Delta \underline{r}^{\prime}\right)\left(\zeta \underline{e}_{r}+\Delta \underline{r}\right) \cdot \underline{e}_{p}^{\prime}}{\zeta^{2}-2 \zeta \Delta r \cos \alpha+(\Delta r)^{2}} .
$$

This function of $\zeta$ is analytic and bounded in the extended plane for $|\zeta|>\Delta \mathrm{r}$.

(c) Next we observe that $\exp [\mathrm{ikp}(\zeta)]$ has an essential singularity at infinity but that the function

$$
e^{i k(\rho-\zeta)}
$$

is analytic and bounded in the extended plane for $|\zeta|>\Delta r$.

Collecting the information in paragraphs (a), (b), and (c), we conclude that the whole expression (9), after multiplication by $\exp (-i k \zeta)$, is analytic in the extended plane for $|\zeta|>\Delta r$ and in fact approaches zero as $|\zeta| \rightarrow \infty$. It, follows that the function $\exp (-i k \zeta) f\left(\underline{e}_{p}, \underline{e}_{p}^{\prime}, \underline{\rho}\right)$ is representable by a Laurent series containing only negative powers of $\zeta$ : Thus we may write

$$
f\left(\underline{e}_{p}, \underline{e}_{p}^{\prime}, \underline{\rho}\right)=e^{i k \zeta} \sum_{n=1}^{\infty} f_{n} \zeta^{-n}
$$


convergent for $|\zeta|\left(=r_{0}\right)>\Delta r$. The $f_{n}$ are in general complicated functions of $\underline{r}$ and $\underline{r}^{\prime}$ (with $\underline{e}_{r_{0}}$ as a parameter). The first coefficient, $f_{1}$, is given explicitly in (23) below.

Example 5.1-1: The argument just used, leading to (14), applies in particular to the function $1 / \rho(\zeta)$ and enables one at once to write the expansion

$$
\frac{1}{\rho(\zeta)}=\frac{1}{\sqrt{\zeta^{2}-2 \zeta \Delta r \cos \alpha+(\Delta r)^{2}}}=\sum_{n=0}^{\infty} h_{n} \zeta^{-n-1},
$$

knowing that it will be convergent for $|\zeta|>\Delta r$. This expansion is indeed well known: the coefficients $h_{n}$ are equal to $(\Delta r){ }^{n} P_{n}(\cos \alpha)$, where $\mathrm{P}_{\mathrm{n}}$ is the $\mathrm{n}^{\text {th }}$ Legendre polynomial.

The series (14) is to be substituted into (8) and integrated term by term (with respect to $\underline{r}$ and $\underline{r}^{\prime}$ ) to obtain the main results (2) and (3). To insure convergence of (14) for all values of $\underline{r}$ and $\underline{r}^{\prime}$ involved in the integrations, we certainly must require

$$
r_{0}>(\Delta r)_{\max }
$$

where $(\Delta r)_{\max }$ denotes the maximum value attainable by $\Delta \mathrm{r}$ as $\underline{r}$ and $\underline{r}^{\prime}$ range independently over their respective finite domains. We find, in fact, that (15) insures uniform convergence of (14) with respect to $\underline{r}$ and $\underline{r}^{\prime}$. This is the key element justifying the term by term integration. Uniform convergence does not follow from point-wise convergence (which we do have), but may be shown by further consideration of the function 


$$
g(\zeta)=e^{-i k \zeta} f\left(\underline{e}_{p}, \underline{e}_{p}^{\prime}, \underline{\rho}\right)
$$

According to the theory of Laurent series

$$
f_{n}=-\frac{1}{2 \pi i} \oint g(\zeta) \zeta^{n-1} d \zeta,
$$

where, for the purpose of this evaluation, we set $\zeta=c_{0} \exp (i \theta)$, with fixed $c_{0}>(\Delta x)_{\max }$. Then

$$
f_{n}=-\frac{c_{0}^{n}}{2 \pi} \int_{0}^{2 \pi} g\left(c_{0} e^{i \theta}\right) e^{i n \theta} d \theta
$$

Further, according to the Cauchy inequality,

$$
\left|f_{n}\right|<c_{0}^{n} M
$$

where $M$ is an upper bound for $\left|g\left(c_{0} e^{i \theta}\right)\right|$ on the (circular) path of integration. Fortunately, from the point of view of the present problem, we can find such an upper bound independent of $\underline{r}$ and $\underline{r}^{\prime}$ for all $\underline{r}$ and $\underline{r}^{\prime}$ involved in the spatial integrations. For arbitrary and independent $\theta$ and $\alpha$, it is easy to show that

$$
\begin{aligned}
& |\underline{\rho}|_{\max }=|\rho|_{\max }=c_{0}+(\Delta r)_{\max }, \\
& |\underline{\rho}|_{\min }=|\rho|_{\min }=c_{0}-(\Delta r)_{\max } .
\end{aligned}
$$

With due care, one finds 


$$
M=8 \pi \frac{|\rho|_{\max }^{2}}{|\rho|_{\text {min }}^{2}}\left(\frac{2}{|\rho|_{\text {min }}^{3}}+\frac{2 k}{|\rho|_{\text {min }}^{2}}+\frac{k^{2}}{|\rho|_{\text {min }}}\right) e^{k\left[|\rho|_{\max }+c_{0}\right]} .
$$

(We have sacrificed some sharpness for the sake of a simpler expression.) Now if $c_{0}$ is chosen between $r_{0}$ and $(\Delta r)_{\max }$,

$$
\mathrm{r}_{0}>c_{0}>(\Delta \mathrm{r})_{\max }
$$

then

$$
\left|f_{n} / \zeta^{n}\right|<\left(c_{0} / r_{0}\right)^{n}{ }_{M}
$$

for all $n \overline{>}$. The quantity on the right is the general term of a convergent series of positive terms; hence, by the familiar Weierstrass M-test, the series in (14) converges uniformly with respect to $\underline{r}$ and $\underline{r}^{\prime}$, as asserted. (Obviously, absolute convergence is confirmed.)

Example 5.1-2: The transmission integra1 for a pair of simp1e, paralle1, line antennas (a 2-dimensional problem) is found by suitable specialization of (50) in Kerns-Dayhoff [21] to be proportional to the Hankel function $\mathrm{H}_{\mathrm{O}}^{(1)}(\mathrm{kd})$. The asymptotic expansion of this function is well known to be divergent. This example shows that divergence may indeed occur when (15) is not fulfilled.

We conclude this subsection with an indication of the origin of the expression (3) for the important first term of the expansion (2). For this purpose we consider $\underline{\underline{\zeta}}=\underline{r}_{0}$. From (9a) with the definition (14) one finds relatively easily 


$$
f_{1}=-k^{-1}\left(\underline{e}_{p} \cdot \underline{e}_{r_{0}} \underline{e}_{r_{0}} \cdot \underline{e}_{p}^{\prime}-\underline{e}_{p} \cdot \underline{e}_{p}^{\prime}\right) \exp \left[i k \underline{e}_{r_{0}} \cdot\left(\underline{r}^{\prime}-\underline{r}\right)\right]
$$

Substitute this quantity into (8a) and factorize the resulting expression according to (4) and (5) evaluated with $\underline{k}=\underline{k}_{0}=\underline{k}_{r_{0}}$. The result is seen to yield (3).

5.2 Expansion of $\exp \left(-2 i k r_{0}\right) \Phi_{00}\left(\underline{r}_{0}\right)$ in Powers of $1 / r_{0}$

In this subsection we show that the reflection integral

$$
\Phi_{00}\left(\underline{r}_{0}\right)=\int \mathrm{d} \underline{K} e^{-i \underline{k}^{-} \cdot \underline{r}_{O}} \underline{s}_{01}(\underline{K}) \cdot \int \underline{s}_{22}^{\prime}(\underline{K}, \underline{L}) \cdot \underline{s}_{10}(\underline{L}) e^{i \underline{\ell}^{\prime} \underline{r}_{O}} d \underline{L}
$$

can be expanded in the asymptotic form

$$
\Phi_{0 O}\left(\underline{r}_{0}\right)=\frac{e^{2 i k r_{0}}}{r_{0}^{2}}\left(C_{20}+C_{21} r_{0}^{-1}+C_{22} r_{o}^{-2}+\ldots\right)
$$

and that convergence of the series is assured provided

$$
\mathrm{r}_{\mathrm{o}}>(\Delta \mathrm{r})_{\max }
$$

where $(\Delta r)_{\max }$ is the same geometrical quantity encountered and defined in the discussion of the expansion of the transmission integral. (See (5.1-15).) Equation (2a) furnishes the Laurent series for $\exp \left(-2 i \mathrm{kr}_{\mathrm{O}}\right) \Phi_{\mathrm{OO}}\left(\underline{\mathrm{r}}_{\mathrm{O}}\right)$ and the representation is not merely asymptotic. 
Sec. 5.2]

The important leading coefficient in (2a) has the expression in terms of PWSM quantities

$$
C_{20}=-\left[2 \pi \gamma\left(K_{0}\right)\right]^{2} \underline{s}_{01}\left(-\underline{K}_{0}\right) \cdot \underline{s}_{2}^{\prime}\left(-\underline{K}_{0}, \underline{K}_{0}\right) \cdot \underline{s}_{10}\left(\underline{K}_{0}\right),
$$

where $\underline{k}_{0}=\underline{k r}_{0} / r_{0}$ and $\underline{k}_{0}$ is the transverse part of $\underline{k}_{0}$. This expression has a qualitative interpretation in the classical radar equation, discussed briefly in section II- 2 .

It will be instructive to begin the argument with the consideration of a very simple case in which the expansion (2a) is almost immediately obtainable-granted the main result of the preceding subsection. Namely, we consider that the scattering object is a conditionally non-scattering antenna provided with a non-reflecting 1oad. (See section 3.) Then the 1eft-side cisscattering will be described directly by the scattering characteristic $\underline{\underline{s}}_{22}^{1}$, which will have the special separable form

$$
\underline{\underline{s}}_{2}^{\prime}(\underline{K}, \underline{L})=-\underline{s}_{2}^{\prime}(\underline{K}) \underline{s}_{02}^{\prime}(\underline{L})
$$

where (as the notation indicates) $\underline{s}_{2}^{\prime} 0$ and $\underline{s}_{0}^{\prime}$ are the pertinent transmitting and receiving characteristics for the antenna. Hence we see that in this case the reflection integral splits into the product of two transmission integrals, one for each direction of energy transit. (Cf. example II-2-1.) Each of the transmission integrals has an expansion of the form of (5.1-2) and the convergence criterion (5.1-15) will apply to each. The 
two expansions may be multiplied together by Cauchy's rule to obtain the expansion (2a) for the case considered, and the same convergence criterion will apply to the product.

Further consideration of the problem of this subsection shows that the essential feature of the special case just solved is not the separability of the scattering characteristic per se, but rather the "double (triple) Fourier transform" representation of the scattering characteristic implicitly involved. The double Fourier-transform representation of a scattering characteristic, say $\underline{\underline{s}}_{22}^{\prime}$, is of the form

$C \gamma_{K}^{-1} \underline{k}^{-} \times\left[\underline{k}^{-} \times \int_{V^{\prime}} \int_{V} \underline{\underline{\Gamma}}\left(\underline{r}, \underline{r}^{\prime} ; \underline{k}^{-}, \underline{\ell}\right) e^{-i \underline{k}} \underline{r}^{-} \underline{r}+i \underline{\ell} \cdot \underline{r}^{\prime} d \underline{r} d \underline{r}^{\prime} \times \underline{\ell}\right] \times \underline{\ell}$,

where the most general $\underline{\underline{\Gamma}}$ is of the form

$\underline{\underline{I}}\left(\underline{\mathrm{r}}, \underline{r}^{\prime} ; \underline{k}, \underline{l}\right)=\underline{\underline{\Gamma}}_{1}\left(\underline{\mathrm{r}}, \underline{r}^{\prime}\right)+\underline{\mathrm{e}}_{\mathrm{k}} \times \underline{\underline{\Gamma}}_{2}\left(\underline{\mathrm{r}}, \underline{r}^{\prime}\right)+\underline{\Gamma}_{3}\left(\underline{\mathrm{r}}, \underline{\underline{r}}^{\prime}\right) \times \underline{\mathrm{e}}_{\ell}+\underline{\mathrm{e}}_{\mathrm{k}} \times \underline{\Gamma}_{4}\left(\underline{\mathrm{r}}, \underline{r}^{\prime}\right) \times \underline{\mathrm{e}}_{l}$

For the special separable case considered above the constant $C$ is equal to $Y_{0}\left(\eta_{0}^{\prime} k\right)^{-1}(4 \pi \varepsilon \omega)^{-2}$, the dyadic $(3 b)$ is equal to $\underline{J}_{e}^{\prime}(\underline{r}) \underline{J}_{e}^{\prime a}\left(\underline{r}^{\prime}\right)$, and the result is obtainable with the aid of (2.2-8) and (1-15). In the general case a representation of the form (3) is obtainable by a more complete argument, which makes explicit use of the fact that the exciting field evoking characteristic scattering is (by definition) a plane wave. In addition to the use of (2.2-7) or (2.2-8), the key element is the timely introduction of suitable Green's functions (see (6)) and the observation that volume inte-

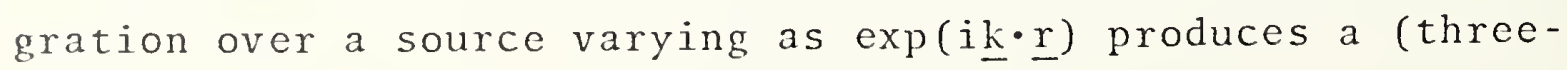


dimensional) Fourier transform (see (8)). The procedure is illustrated in the following paragraphs for a relatively simple case.

For definiteness and relative simplicity, we consider the case of scattering by an inhomogeneous and finitely conducting, non-magnetic obstacle. The obstacle is conveniently characterized by a complex susceptibility $\underline{\underline{x}}=\left(\underline{\underline{\varepsilon}}-\varepsilon_{\mathrm{O}} \underline{\underline{1}}\right) / \varepsilon_{\mathrm{O}}$, which is to differ from zero only in a finite region V. From (2.1-6) we obtain by rearrangement of the second equation

$$
\left.\begin{array}{c}
\nabla \times \underline{E}_{i}-i \omega \underline{B}_{i}=0 \\
\underline{B}_{i}+i \omega \underline{\underline{\varepsilon}} \cdot \underline{E}_{i}=\underline{J}_{e}^{\prime},
\end{array}\right\}
$$

where $\underline{J}_{e}^{\prime} \equiv-i \omega \varepsilon_{0} \underline{x}^{\prime} \underline{E}_{0}$. This rearrangement converts the problem to one with a known current density vector, which in particular vanishes outside $V$, as the source of the fields in the inhomogeneous domain. Thus we can write for the electric field, incident plus induced,

$$
\begin{aligned}
\underline{E}(\underline{r}) & =\underline{E}_{O}(\underline{r})+\int_{V} \underline{\underline{G}}\left(\underline{r}, \underline{r}^{\prime}\right) \cdot \underline{J}_{e}^{\prime}\left(\underline{r}^{\prime}\right) d \underline{r}^{\prime} \\
& =\underline{E}_{O}(\underline{r})-i \omega \varepsilon_{0} \int_{V} \underline{\underline{G}}\left(\underline{r}, \underline{r}^{\prime}\right) \cdot \underline{x}\left(\underline{r}^{\prime}\right) \cdot \underline{E}_{O}\left(\underline{r}^{\prime}\right) d \underline{r}^{\prime} .
\end{aligned}
$$

The important thing about this expression for our purposes is the linearity with respect to $\underline{E}_{0}$. (Judging from the results of Yaghjian [6] for the case of a homogeneous domain, the volume integral in (5) is defined only after the choice of a "principal volume" and the tensor $\underline{\underline{G}}$ must contain an additive term depending upon the choice of that volume. Cf. also remarks in example $2.2-2(b)$. 
The $\underline{J}_{\mathrm{e}}$ of $(2.1-6)$ is given by $-i \omega \varepsilon_{\mathrm{O}} \underline{x} \cdot \underline{E}(\underline{r})$; combining this and (5), we can write

$$
\underline{J}_{e}(\underline{r})=\int_{V} \underline{\underline{\Gamma}}\left(\underline{r}, \underline{r}^{\prime}\right) \cdot \underline{E}_{0}\left(\underline{r}^{\prime}\right) d \underline{r}^{\prime}
$$

where $\underline{\underline{\Gamma}}$ is the appropriate kernel (it must include a diagonal transformation to account for $\underline{E}_{0}$ in (5)). We now synthesize an expression for $\underline{\underline{s}}_{11}$ as defined in (2.2-12a), using (6), (2.2-7), and the prescribed TM and TE incident fields, which have

$$
\underline{E}_{0}(\underline{r})=\underline{e}_{\|}\left(\underline{l}^{-}\right) e^{i} \underline{\ell}^{-} \cdot \underline{r} /(2 \pi)
$$

and

$$
\underline{E}_{0}(\underline{r})=\underline{e} \perp\left(\underline{\ell}^{-}\right) e^{i \underline{\ell^{-}} \cdot \underline{r}} /(2 \pi)
$$

respectively. Clearly we have

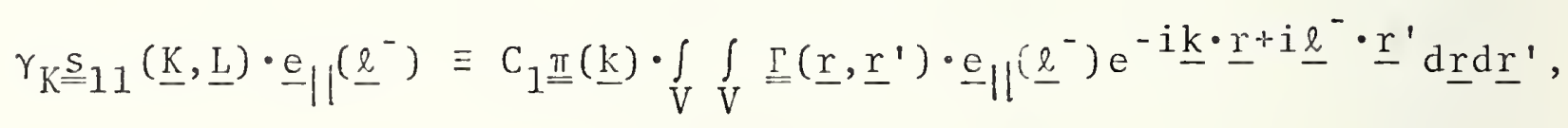

where $C_{1}=-\omega \mu /\left(8 \pi^{2}\right)$, as well as the corresponding equation with $\underline{\mathrm{e}} \mathbf{1}$ replacing $\underline{\mathrm{e}} \|$. We can secure the required

$$
\underline{S}_{11}(\underline{K}, \underline{L}) \cdot \underline{\ell}^{-}=0
$$

without invalidating (8a) by setting

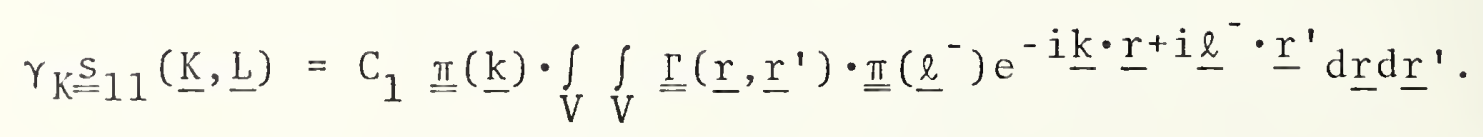


Sec. 5.2]

This exibits $\underline{\underline{s}}_{11}$ in the desired form.

It is an expected (but for the present derivation incidenta1) corollary of (9) that the reciprocity rolation for $\underline{\underline{r}}$ implies that for $\underline{\underline{s}}_{11}$; that is, $\underline{\underline{\Gamma}}\left(\underline{r}^{\prime}, \underline{r}\right)=\tilde{\Gamma}^{\mathrm{r}}\left(\underline{r}, \underline{r}^{\prime}\right)$ implies $(1-16)$.

We assemble the quantities needed for the consideration of the reflection integral (1), considering a "transceiver" to be on the left and a scattering object to be on the right. For a scattering object on the right, we obtain the needed expression

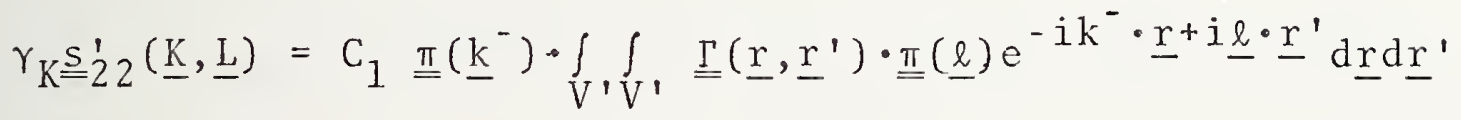

by an obvious revision of (9). For the transceiver we need

$$
\gamma_{L} \underline{S}_{10}(\underline{L})=\frac{-\omega \mu}{4 \pi} \underline{\underline{\pi}}(\underline{l}) \cdot \int_{V} \underline{J}_{\mathrm{e}}(\underline{r}) \mathrm{e}^{-i \underline{\ell} \cdot \underline{r}} \mathrm{~d} \underline{\underline{r}},
$$

which may be obtained from $(2.2-7)$, and we need

$$
\underline{s}_{01}(\underline{K})=\frac{-1}{4 \pi \eta_{0}} \underline{\underline{\pi}}\left(\underline{k}^{-}\right) \cdot \int_{V} \underline{J}^{a}(\underline{r}) e^{i \underline{k}-} \cdot \underline{r} d \underline{r},
$$

which may be obtained with the aid of reciprocity in the form (1-15). We substitute these expressions into the reflection integral and arrange the integrations to obtain

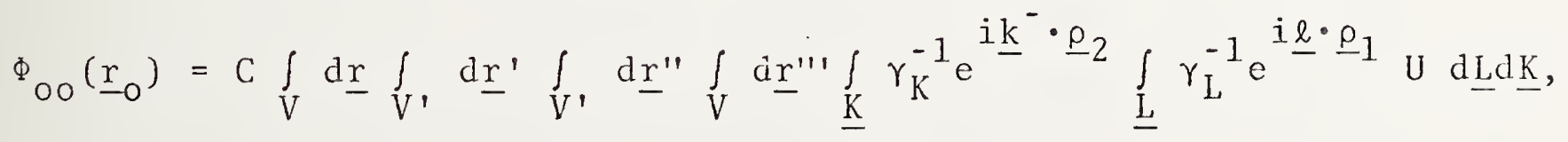


where C absorbs all multipliers, and we have defined the vectors

$$
\underline{\rho}_{1}=\underline{r}^{\prime \prime}-\underline{r}+\underline{r}_{0}, \quad \underline{\rho}_{2}=\underline{r}^{\prime \prime}-\underline{r}^{\prime}-\underline{r}_{0},
$$

which are pictured in figure 3. Further, with the aid of the definition (2.2-9), the symmetry, and the idempotent property (1-6a) of $\underline{\underline{\pi}}$, the quantity $U$ appearing in (13a) can be put in the form

$$
U=\sum_{x, y} \underline{J}_{e}^{a}\left(\underline{r}^{\prime \prime \prime}\right) \cdot\left(k^{2} \underline{\underline{1}}-\underline{k k}\right) \cdot \underline{e}_{x} \Gamma_{x y}\left(\underline{r}^{\prime}, \underline{r}^{\prime \prime}\right) \underline{e}_{y} \cdot\left(k^{2} \underline{\underline{1}}-\underline{\ell l}\right) \cdot \underline{J}_{e}(\underline{r}) \cdot
$$

Here the $\Gamma_{x y}$ are Cartesian components of $\underline{\underline{\Gamma}}$, and $x, y$ used as summation indices independently take on the values $x, y, z$. The $\underline{K}$ and $\underline{L}$ integrations may now be performed; one obtains (apart from multipliers)

$$
\left|\underline{J}_{e}^{a}\left(\underline{r}^{\prime \prime \prime}\right)\right|\left|\underline{J}_{e}(\underline{r})\right| \sum_{x, y} f\left(\underline{e}_{p}^{a}, \underline{e}_{x}, \underline{\rho}_{2}\right) \Gamma_{x y}\left(\underline{r}^{\prime}, \underline{r}^{\prime \prime}\right) f\left(\underline{e}_{p}, \underline{e}_{y}, \underline{\rho}_{1}\right),
$$

where $f$ is the dipole-dipole coupling function given in (5.1-9) and $\underline{e}_{p}^{a}$ and $\underline{e}_{p}$ are respectively 


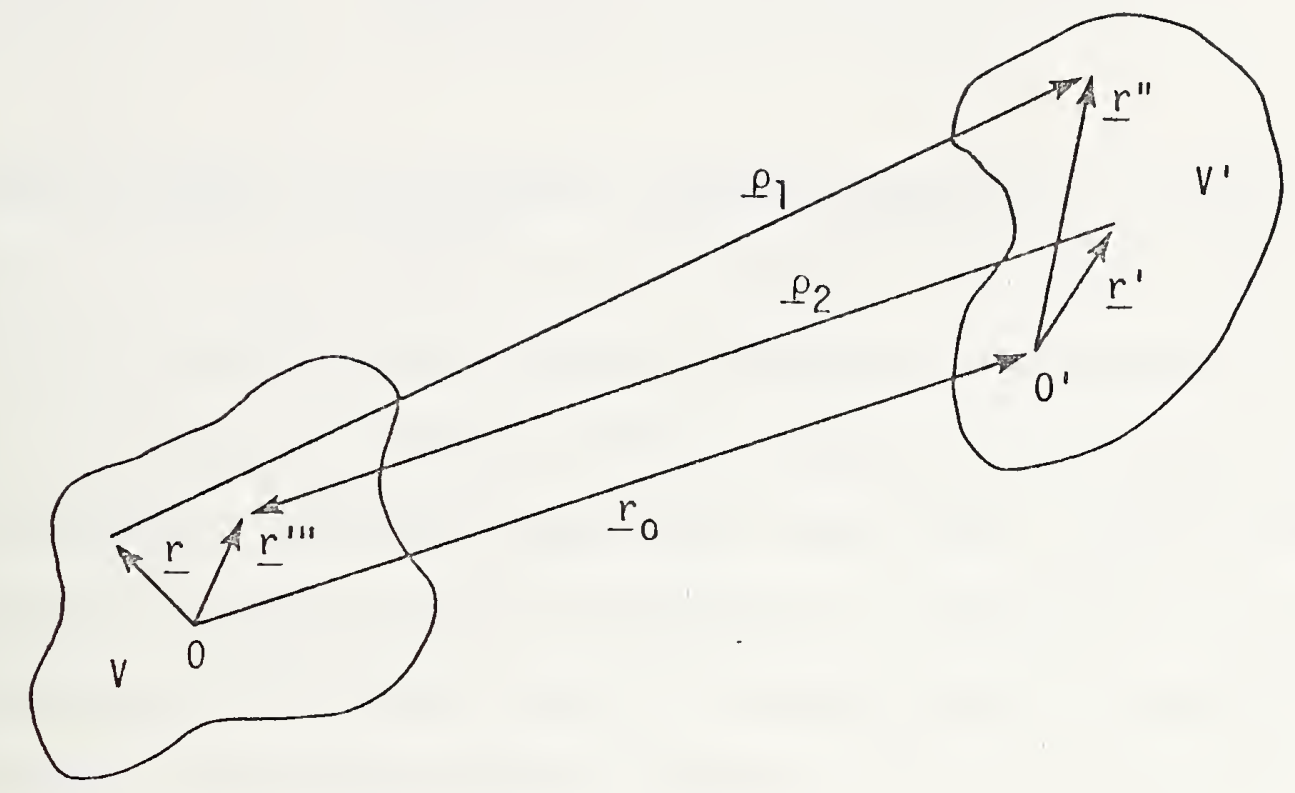

Figure 3. The vectors appearing in (5.2-13b).

the (Hermitian) unit vectors associated with $\underline{J}_{e}^{a}$ and $\underline{J}_{e}$ (cf. $(5.1-7))$. The remainder of the argument is essentially an application of the main results of the preceding subsection to the case in which we have products of pairs of $f$-functions, similar to the case of separable scattering discussed at the beginning of this subsection. Consideration of the definitions and the roles of $\underline{\rho}_{1}$ and $\underline{\rho}_{2}$ shows that $(\Delta \mathrm{r})_{\max }$, in the sense of (5.1-15), is the same for both $\underline{\rho}_{1}$ and $\underline{\rho}_{2}$. We now require that (2b), i.e., $r_{0}>(\Delta r)_{\max }$, be in force. Then each of the functions of the form $f\left(\underline{e}_{p}, \underline{e}_{y}, \underline{\rho}_{2}\right)$ is expressible in the form of (5.1-14), with the convergence properties already noted. The several products of pairs of series in each term of (15) may be multiplied by Cauchy's rule and summed over $x, y$ to yield a single series of the form 


$$
e^{2 i k r} 0 \sum_{n=2}^{\infty} f_{n}\left(\underline{r}, \underline{r}^{\prime}, \underline{r}^{\prime \prime}, \underline{r}^{\prime \prime \prime}\right) / r_{0}^{n},
$$

with persistence of the convergence properties. That is, the series in (16) converges uniformly with respect to $\underline{r}, \underline{r}^{\prime}, \underline{r}^{\prime \prime}$, and $\underline{r}^{\prime \prime}$ for all values of these quantities involved. (In (16) we also have uniform convergence with respect to $r_{0}$ and absolute convergence, but at this stage these properties are somewhat incidental.) Completion of the evaluation of (13a) by term-byterm integration of (16) yields (2a) and completes the argument. The method of this derivation extends readily to higher iterated integrals in the Liouville-Neumann series. Indeed, the representations for $\hat{\mathrm{S}}_{10}, \hat{\mathrm{S}}_{11}, \hat{\mathrm{S}}_{22}^{\prime}, \hat{\mathrm{S}}_{01}$, and $\hat{\mathrm{S}}_{02}$ set up in this and the preceding subsection are suitable and sufficient for the consideration of all the iterated integrals involved in the Liouville-Neumann series for $M_{00}$ and $M_{0^{\prime}}$ (ref. (II-2-11, -12)). One must, of course, construct more and more complicated vector diagrams of the type shown in figures 2 and 3 . The asymptotic expansion of an integral associated with $m$ transits (or $m-1$ reflections) of a wave is found to be of the form

$$
\frac{e^{i m k r_{0}}}{r_{0}^{m}}\left(C_{m o}+C_{m 1} r_{o}^{-1}+C_{m 2} r_{o}^{-2}+\ldots\right) .
$$

This result has been given by Wacker [18] for the on-axis case $\left(\underline{r}_{0}=d \underline{e}_{z}\right)$. The method of the present section shows that the convergence criterion for (17) is the same as that already found in the two cases that we have considered explicitly. 


\section{APPENDICES TO CHAPTER III}

\subsection{Appendix A: Ideal Electric-Probe Response}

In this appendix we obtain the result, already quoted in example 2.2-8(e), for the receiving response of a reciprocal, lossless antenna having a spectrum identical to that of an elementary electric dipole. In example 2.2-8(d) we found that the receiving characteristic of such an antenna, situated at the origin $0^{\prime}$ of its own fiducial coordinate system, can be expressed as

$$
\underline{\mathrm{s}}_{\mathrm{oq}}(\underline{K})=C \underline{\mathrm{e}}_{\mathrm{p}}^{\prime} \cdot \underline{\underline{\pi}}(\underline{\mathrm{k}}),
$$

where $e_{-p}^{\prime}$ is a fixed, real, unit vector and $|C|$ is a determinate constant. The receiving antenna (thus $0^{\prime}$ ) is at the point $\underline{r}_{O}$ in the fiducial coordinate system Oxyz of the source.

For the moment we assume that the source of the incident field is located entirely to the left of the receiving antenna. Then from the definitions of the PWSM quantities (e.g., in (1-3a)) we find

$$
\mathrm{b}_{0}^{\prime}\left(\underline{r}_{0}\right)=F^{\prime} \int \underline{s}_{0}^{\prime}(\underline{K}) \cdot \underline{a}_{2}^{\prime}(\underline{K}) d \underline{K},
$$

where $F^{\prime}=\left(1-S_{O O}^{\prime} \Gamma_{L}\right)^{-1}$. The incident spectrum $\underline{a}_{2}^{\prime}(\underline{K})$ is related to the emergent spectrum $\underline{b}_{1}(\underline{K})$ of the source by $(1-13)$; hence we have 


$$
\mathrm{b}_{0}^{\prime}\left(\underline{r}_{0}\right)=F^{\prime} \int \underline{s}_{0}^{\prime}(\underline{K}) \cdot \underline{b}_{1}(\underline{K}) e^{i \underline{k} \cdot \underline{r}_{0}} d \underline{K} .
$$

For the special receiving characteristic (1), (3) becomes

$$
b_{0}^{\prime}\left(\underline{r}_{0}\right)=F^{\prime} \underline{e}_{-p}^{\prime} \cdot \int \underline{b}_{1}(\underline{K}) e^{i \underline{k} \cdot \underline{r}_{0}} d \underline{K}
$$

by $(I I-1.2-10)$ the integral in $(4 a)$ is just $2 \pi \underline{E}\left(\underline{r}_{0}\right)$. Thus

$$
b_{0}^{\prime}\left(\underline{r}_{0}\right)=2 \pi F^{\prime} \underline{e}_{p}^{\prime} \cdot \underline{E}\left(\underline{r}_{0}\right) .
$$

It is clear that $\underline{E}$ may be any field due to sources on the left-a transmitting antenna, a scattering object, or simply a prescribed current distribution in V. Furthermore, it is easily verified that ( $4 \mathrm{~b})$ continues to hold for any incident field $\underline{E}$ due to sources simultaneously present on both the left and the right.

Finally we note that $(4 b)$ actually holds for all points "outside" source distributions (where $\underline{J}_{e}=0$ ); that is, the "noencroachment" rule need not be in effect and the probe may be located in a z-plane cutting through the source distribution. The proof can easily be carried out with the use of the cumulative spectra shown in example 2.2-2(b). Of course, this result is not unexpected. 
Sec. 6.2]

6.2 Appendix B: Relation between Spectrum and Asymptotic E

Part of the purpose of this appendix is the derivation of the relation bctween the far-field values of a scattered or radiated field and its plane-wave spectrum. The relation is stated in (II-1.2-16) and is, of course, of essential interest at a number of points in this monograph. It can be derived (apart from an estimate of the remainder) by adaptation of an argument given (for a different physical problem) in Born and Wolf [22], p. 750, and by a Fourier transform method given by Yaghjian [25]. However, it is also part of our purpose to obtain the relation in question (including an estimate of the remainder) as a special case or corollary of results already obtained in this monograph. In the process, we incidentally obtain an expansion theorem for the electromagnetic field of a bounded source distribution, of a type given by a number of authors, notably wilcox [23](where additional references may be found).

Our measurement-biased approach may afford an interesting and informative constrast to the more mathematical point of view, under which $\underline{E}$ is a rather abstract entity, not usually considered subject to the difficulties of measurement.

We draw mainly upon the results of subsection 5.1 regarding transmission between two antennas and specialize those results by assuming that the receiving antenna is an "ideal" probe, of the type specified and treated in appendix A. Thus the expressions for the characteristics $(A-1)$ and for the response $(A-4 b)$ will be applicable in what follows; with the advantage, as it turns out, 
that the specific value of $C$ is immaterial. We might further assume that the receiving antenna is a critically-1oaded, conditionally non-scattering antenna, so that there will be no scattering from the probe--hence no multiple reflections--to complicate the picture. Alternatively and more conventionally, we simply assume that the sources of $\underline{E}$ are fixed.

The arrangement considered is similar to that of subsection 5.1. The current distribution generating the field to be probed is wholly contained in the finite region $V$ (fig. 2); the region $V^{\prime}$ of the probe is represented by a single point at $\underline{r}_{0}$. The plan of the derivation is simply to equate alternative expressions for $b_{0}^{\prime}\left(\underline{r}_{0}\right)$, one given by $(A-4 b)$ and the other coming from subsection 5.1 .

If, in particular, the source is the normalized equivalent current density $\underline{J}_{e}$ of a transmitting antenna, we have (in the assumed absence of multiple reflections), $b_{0}^{\prime}\left(\underline{r}_{0}\right)=a_{0} F^{\prime} \Psi_{0}{ }_{0}\left(\underline{r}_{0}\right)$, with a series evaluation of $\Psi_{0^{\prime}}{ }^{\left(r_{0}\right)}$ given directly by (5.1-2). However, we wish to modify the meaning and the form of that expression to accommodate more general source currents and to exploit the simple properties of the special receiving characteristic. It is essential to recall that $\Psi_{0}{ }_{0}\left(\underline{r}_{0}\right)$ is bilinear in $\underline{J}_{e}$ and $\underline{J}_{e}^{a}$. The multiplier a may be associated with $\underline{J}_{e}$ to produce, in the first instance, the un-normalized equivalent current-density, $\underline{J}_{e}=a_{0} \underline{J}_{e}$, for a transmitting antenna. But we recognize that $\underline{J}_{e}$ may equally well represent the un-normalized equivalent current-density for a scatterer--or just a prescribed 
current density in a finite region V. Furthermore, the factor $\mathrm{Ce}_{\mathrm{p}}^{\prime}$ in $(\mathrm{A}-1)$ is seen to be contained as a multiplier in (5.1-8a) and hence in all the terms of $(5.1-2)$. These considerations make the following change of notation appropriate: We set

$$
a_{0} C_{1 n}=2 \pi C_{-p}^{p} \cdot A_{n},
$$

where the $2 \pi$ is inserted in anticipation of a later cancellation, and the $\underline{A}_{n}$ give effect to the broadened interpretation of $\underline{J}_{e}$. Thus we have

$$
b_{0}^{\prime}\left(\underline{r}_{0}\right)=\frac{e^{i k r_{0}}}{r_{0}} 2 \pi C F^{\prime} \underline{e}_{p}^{\prime} \cdot \sum_{n=0}^{\infty} \underline{A}_{n} r_{0}^{-n} .
$$

Equating (2) and $(\mathrm{A}-4 \mathrm{~b})$ and canceling the common factors (including the directionally arbitrary $\underline{-}_{p}^{\prime}$ ), we obtain

$$
\underline{E}\left(\underline{r}_{0}\right)=\frac{e^{i k r_{0}}}{r_{0}} \sum_{n=0}^{\infty} \underline{A}_{n} r_{0}^{-n} \text {, }
$$

with convergence assured provided

$$
r_{0}>r_{\max }
$$

where $r_{\max }$ denotes the maximum (or an upper bound) for $r$ with $\underline{r}$ in $V$. Although we have considered transmission to be nominally from left to right, there is of course no preferred (hemispherical) direction of transmission. Hence (3) holds for any direction of $\underline{r}_{0}$, and the result coincides with that given by wilcox for bounded scatterers [23]. 
The result of main interest in this appendix is a corollary of (3). With the aid of (1) and the expression (5.1-3) for $\mathrm{C}_{10}$, we see that $\underline{A}_{0}$ must equal $-i \gamma\left(K_{0}\right) \underline{b}_{q}\left(\underline{K}_{0}\right)$. Hence we have established

$$
\underline{E}_{q}\left(\underline{r}_{0}\right)=-i \gamma\left(K_{0}\right) \underline{b}_{q}\left(\underline{K}_{0}\right) e^{i k r_{0}} / r_{0}+0\left(r_{0}^{-2}\right)
$$

where $\underline{K}_{0}=\underline{R}_{0} k / r_{0}$. This is just (II-1.2-16b) provided with an order-of-magnitude remainder term. 


\section{REFERENCES}

[1] Kerns, D. M., Plane-wave scattering-matrix and generalized reciprocity relations for antennas and scatterers, NBS Report (unpublished) (June 1972).

[2] Kerns, D. M., Plane-wave spectra and spectral coupling products for arbitrary radiating and scattering systems, NBS Report (unpublished) (March 1972).

[3] Gibbs, J. W., and Wilson, E. B., Vector Analysis (Dover, New York, 1960).

[4] Stratton, J. A., Electromagnetic Theory (McGraw-Hill, New York, 1941).

[5] Brekhovskikh, L. M., Waves in Layered Media (Trans. from Russian by David Lieberman) (Academic Press, 1960).

[6] Yaghjian, A. D., A direct approach to the derivation of electric dyadic Green's functions, Symposium Digest, IEEE/AP-S International Symposium, Amherst, Mass., 71-73 (October 11-15, 1976). For the complete paper see Nat. Bur. Stds. (U.S.) Tech. Note 1000 (January 1978).

[7] Schelkunoff, S. A., Electromagnetic Waves (D. Van Nostrand, New York, 1943).

[8] Montgomery, C. G., Dicke, R. H., and Purcell, E. M., (Eds.), Principles of Microwave Circuits (McGraw-Hill, New York, $1948)$.

[9] Kahn, W. K., and Kurss, H., Minimum-scattering antennas, IEEE Trans. Antennas and Propagat. AP-13, 671-675 (September 1965). 
[10] Wasylkiwskyj, W., and Kahn, W. K., Theory of coupling among minimum-scattering antennas, IEEE Trans. Antennas and Propagat. AP-18,204-216 (March 1970).

[11] Kraus, J. D., Antennas (McGraw-Hill, New York, 1950).

[12] Courant, R., and Hilbert, D., Methods of Mathematical Physics (Interscience, New York, 1953).

[13] Riesz, F., and Sz.Nagy, B., Functional Analysis (Ungar Publishing Co., New York, 1955).

[14] Richmond, J. H., A modulated scattering technique for measurement of field distributions, IRE Trans. Microwave Theory Tech. MTT-3, 13-15 (July 1955).

[15] Justice, R., and Rumsey, V. H., Measurement of electric field distributions, IRE Trans. Antennas Propagat. AP-3, 177-180 (October 1955).

[16] Cullen, A. L., and Parr, J. C., A new perturbation method for measuring microwave fields in free space, Proc. Inst. Elec. Eng. 102B, 836-844 (November 1955).

[17] Harrington, R. F., Small resonant scatterers and their use for field measurements, IRE Trans. Microwave Theory Tech. MTT-10, 165-174 (May 1962).

[18] Wacker, P. F., Theory and numerical techniques for accurate extrapolation of near-zone antenna and scattering measurements, NBS Report (unpublished) (April 1972).

[19] Newel1, A. C., Baird, R. C., and Wacker, P. F., Accurate measurement of antenna gain and polarization at reduced distances by an extrapolation technique, IEEE Trans. Antennas Propagat. AP-21, 418-431 (July 1973). 
[20] Newel1, A. C., and Kerns, D. M., Determination of both polarisation and power gain of antennas by a generalised 3-antenna measurement method, Electron. Lett. 7, 68-70 (February 1971).

[21] Kerns, D. M., and Dayhoff, E. S., Theory of diffraction in microwave interferometry, J. Res. Nat. Bur. Stand. (U.S.) 64B, 1-13 (January-March 1960).

[22] Born, M., and Wolf, E., Principles of Optics (Pergamon. Press, London, 1959).

[23] Wilcox, C. H., An expansion theorem for electromagnetic fields, Communications on Pure and Applied Mathematics IX, $115-134$ (May 1956).

[24] Erdélyi, A., Asymptotic Expansions (Dover, New York, 1956).

[25] Yaghjian, Arthur D., Near-field antenna measurements on a cylindrical surface, Nat. Bur. Stds. (U.S.) Tech. Note 696 (revised Sept. 1977). 
NBS-iILA (REV. 7.73)

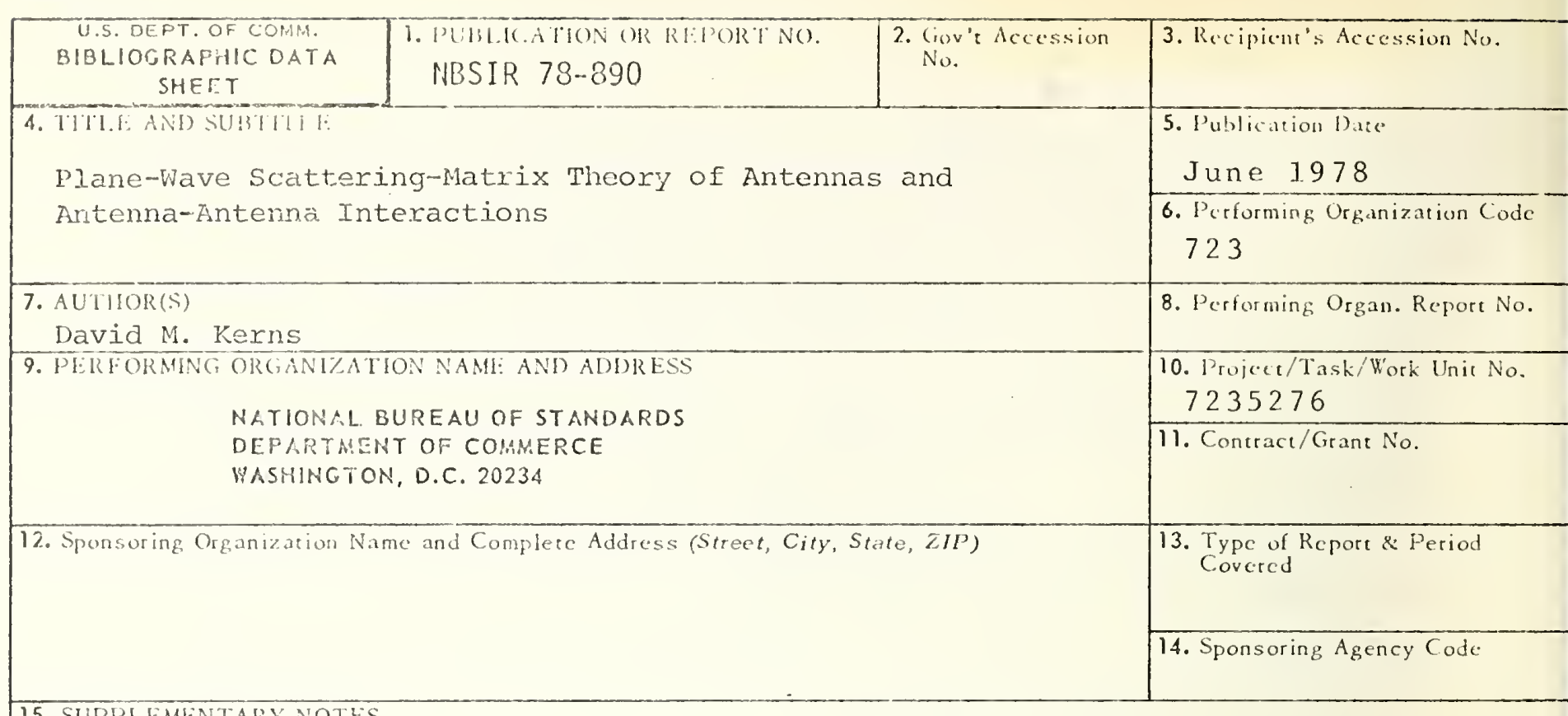

15. SUPPLEMENTARY NOTES

16. ABSTRACT (A 200-word or less factual summary of most significant information. If clocument includes a significant

bibliography or literature survey, mention it here.)

This monograph is distinguished by the use of plane-wave spectra for the representation of fields in space and by the consideration of antenna-anienna (and antenna-scatterer) interactions at arbitrary separation distances. The plane-wave representation is eminentII suitable for this purpose as well as for the expression of conventional asymptotic quantities of antenna theory, such as power gain, effective area, and polarization. The primary objective of the monograph is to facilitate the critical acceptance and proper application of antenna and field measurements techniques deriving more or less directiy from the plane-wave scattering-matrix (PrSM) theory of antennas and antenna interactions. A secondary objective is to present some recent and some new theoretical results based on this theory. The three chapiers of this monograph provide (a) an introduction to the basic theory and practice of microwave network analysis (which form an inescopable part of microwave antenna measurement expertise); (b) a thorough formulation of the PWSM theory of antennas and antenna-antenna interactions, including analytical techniques for derived measurement methods; and (c) recent and new theoretical results and analytical examples. Topics include: a convenient and attractive reformulation of the PISM theory, some theory of minimum scattering antennas; theory of classes of completely solvable antenna-antenna interaction problems; and convergent, asymptotic expansions of transmission- and reflection-integrals in reciprocal powers of r , where r is the magitude of the relative displacement vector $\underline{r}_{0}$ and the direction of $\underline{r}_{0}$ is a
parameter.

17. KEY WORDS (six to twelve entries; alphabetical order; capitalize only the first letter of the first key word unless a proper name; separated by semicolons)

Antenna-antenna systems; antenna measurements; antenna-scatterer systems; antenna theory; nicrowave antenna theory; scattering-matrix description of antennas

18. AVALA.ABHLITY

[- Unlimited

D. For Official Distribution. Do Not Release to NTIS

[.7 Order From Sup of Doc, U.S. Govermment Printing Office Wathingon, B.C. 20.102, sh Cac. No. \{1?

Order Fromi National Technical lintermatuon Service (NTIS) Snimplicht, V'irgonia 22151

\begin{tabular}{|l|l|}
\hline $\begin{array}{l}\text { 19. SECURITY CLASS } \\
\text { (THIS REPURT) } \\
\text { UNCL ASSIFHED }\end{array}$ & 21. NO. OF PAGES \\
\hline $\begin{array}{l}\text { 20. SIECURITY CLASS } \\
\text { (THIS PAGE) }\end{array}$ & 22. PriCe \\
UNCI.ASSIIIII) & USCOMM-OC 29042-P74
\end{tabular}



